University of Louisville

ThinkIR: The University of Louisville's Institutional Repository

$12-2016$

\title{
"If this stuff matters, why isn't it being shared?" : citations, hyperlinks, and potential public futures of online writing in rhetoric and composition.
}

Elizabeth Frances Bergeron Chamberlain

University of Louisville

Follow this and additional works at: https://ir.library.louisville.edu/etd

Part of the Rhetoric and Composition Commons

\section{Recommended Citation}

Chamberlain, Elizabeth Frances Bergeron, "'If this stuff matters, why isn't it being shared?" : citations, hyperlinks, and potential public futures of online writing in rhetoric and composition." (2016). Electronic Theses and Dissertations. Paper 2598.

https://doi.org/10.18297/etd/2598

This Doctoral Dissertation is brought to you for free and open access by ThinkIR: The University of Louisville's Institutional Repository. It has been accepted for inclusion in Electronic Theses and Dissertations by an authorized administrator of ThinkIR: The University of Louisville's Institutional Repository. This title appears here courtesy of the author, who has retained all other copyrights. For more information, please contact thinkir@louisville.edu. 
"IF THIS STUFF MATTERS, WHY ISN'T IT BEING SHARED?": CITATIONS, HYPERLINKS, AND POTENTIAL PUBLIC FUTURES OF ONLINE WRITING IN RHETORIC AND COMPOSITION

By

Elizabeth Frances Bergeron Chamberlain

B.A., California State University, Los Angeles, 2007

M.A., California Polytechnic State University, San Luis Obispo, 2010

A Dissertation

Submitted to the Faculty of the

College of Arts and Sciences of the University of Louisville

in Partial Fulfillment of the Requirements

for the Degree of

Doctor of Philosophy

in English/Rhetoric and Composition

Department of English

University of Louisville

Louisville, Kentucky

December 2016 
Creative Commons Attribution (CC BY 3.0 US) 2016 by Elizabeth Frances Bergeron Chamberlain

Some Rights Reserved 

"IF THIS STUFF MATTERS, WHY ISN'T IT BEING SHARED?": CITATIONS, HYPERLINKS, AND POTENTIAL PUBLIC FUTURES OF ONLINE WRITING IN RHETORIC AND COMPOSITION

\author{
By \\ Elizabeth Frances Bergeron Chamberlain \\ B.A., California State University, Los Angeles, 2007 \\ M.A., California Polytechnic State University, San Luis Obispo, 2010 \\ A Dissertation Approved on
}

November 21, 2016

by the following Dissertation Committee:

Dr. Mary P. Sheridan, Director

Dr. Bronwyn Williams

Dr. Brenda Brueggemann

Dr. Carolyn R. Miller

Dr. Olfa Nasraoui 


\section{DEDICATION}

To the seventeen generous writers and editors who let me poke their writing, pick their brains, and eke a dissertation out of it. 


\section{ACKNOWLEDGEMENTS}

Without Mary P. Sheridan's unflagging confidence that I would emerge victorious, with interesting findings, from the methodological mess of this project, I would doubtless have put much of it aside long ago. If this dissertation at any point seems to evidence internal coherence or an argument for its disciplinary stakes, know that you have seen Mary P.'s hand. Thanks, too, to the rest of my committee: Bronwyn Williams's healthy skepticism pushed me to rethink assumptions; Brenda Brueggemann's fiery candor mentored me through my middle years in Louisville; Carolyn R. Miller's meticulous attention to terms helped me work past many a conflation; and Olfa Nasraoui's guidance at the head of this project gave it shape. Without my writing group, and their patience and enthusiasm and support, this dissertation would have moldered in the anxious recesses of my mind - so deep thanks to those four brilliant women, each of whom I am sure will soon make me proud to say, "I knew her when": Rachel Gramer, Megan Faver Hartline, Keri Mathis, and Laura Tetreault. Their emotional support, alongside support from good friends including Jamila Kareem, helped me see light not just at the end of the tunnel but right there in the middle of it.

My motley crew of parental figures and other family members has led the cheer from the left coast: Thanks to my dad, Brandt, whose successful dissertating when I was in grade school inspired me to one day submit myself to similar madness (and whose knowledgeable advice along the way proved invaluable). Thanks to my mom, Cyndi, who taught me early that brilliant, career-driven, and family-oriented were not exclusive categories. And thanks to my stepmom, Patti; stepsister, Camryn; and my parents-in-law, Kathy and Steve, all four of whom have championed and helped me believe in my potential for success. Yet no one has been more my champion than my brother, Etienne, whose daily phone calls ground me when I am otherwise floundering and whose devotion to his own dreams inspires conviction.

And I could never adequately thank my husband, Sterling, who has let me drag him north and east and deep into the south, following my academic dreams. Who said, "I'll stay up with you," through nearly every night of writing or revising. Who said, "Talk me through it," any time I complained of feeling stuck. Whose love and stability and humor and intelligence have been the defining features of home through four dwellings, in three states, over nearly a decade. 


\begin{abstract}
“IF THIS STUFF MATTERS, WHY ISN'T IT BEING SHARED?”: CITATIONS, HYPERLINKS, AND POTENTIAL PUBLIC FUTURES OF ONLINE WRITING IN RHETORIC AND COMPOSITION
\end{abstract}

\author{
Elizabeth Frances Bergeron Chamberlain
}

November 21, 2016

This dissertation addresses two deceptively discrete questions: (1) how academics might reach wider public audiences, and (2) how and why people cite the way they do. It takes citation practices as a telling though often tacit practice, one through which it is possible trace the contours of a larger story about how writing is changing as it moves online. That story: Writers increasingly reflect goals of provocation, of attracting a wider and potentially global audience, of spreading a message rapidly and virally, of responding to recent events and conversations, of sharing sources and resources.

To explore these questions, this dissertation forwards a mixed-methods study of citation and writing practices in three different sites: In popular press web writing (on Slate and Newsweek - Chapter II), in traditional academic print text in rhetoric and composition (in CCC and College English-Chapter III), and in academic webtext online (on Kairos and Computers and Composition Online-Chapter IV). Chapter II conducts a rhetorical corpus analysis of Slate and Newsweek, seeking transcendent citation practices 
within each journal and considering how those practices (and other writing practices) and others correlate (or not) with social sharing; I then report on interviews with authors from Slate, aiming to elucidate those findings. Chapter III conducts a rhetorical corpus analysis of $C C C$ and College English, seeking an understanding of citation practices in the field of rhetoric and composition more traditionally, more historically; as in the previous chapter, these findings are commented upon and elucidated by authors/editors of each journal. Chapter IV considers hyperlink and parenthetical citation practices in webtext journals Kairos and Computers and Composition Online, via discourse-based interviews with several authors and editors for each journal. Chapter V draws parallels among my investigations and ultimately concludes with a proposal for a new kind of hytpertextual academic publication aimed at "the public"; it offers, at its close, some documents intended to sketch the shape of such a publication, including a "Rhetoric of Hypermedia" style guide for authors. 
TABLE OF CONTENTS

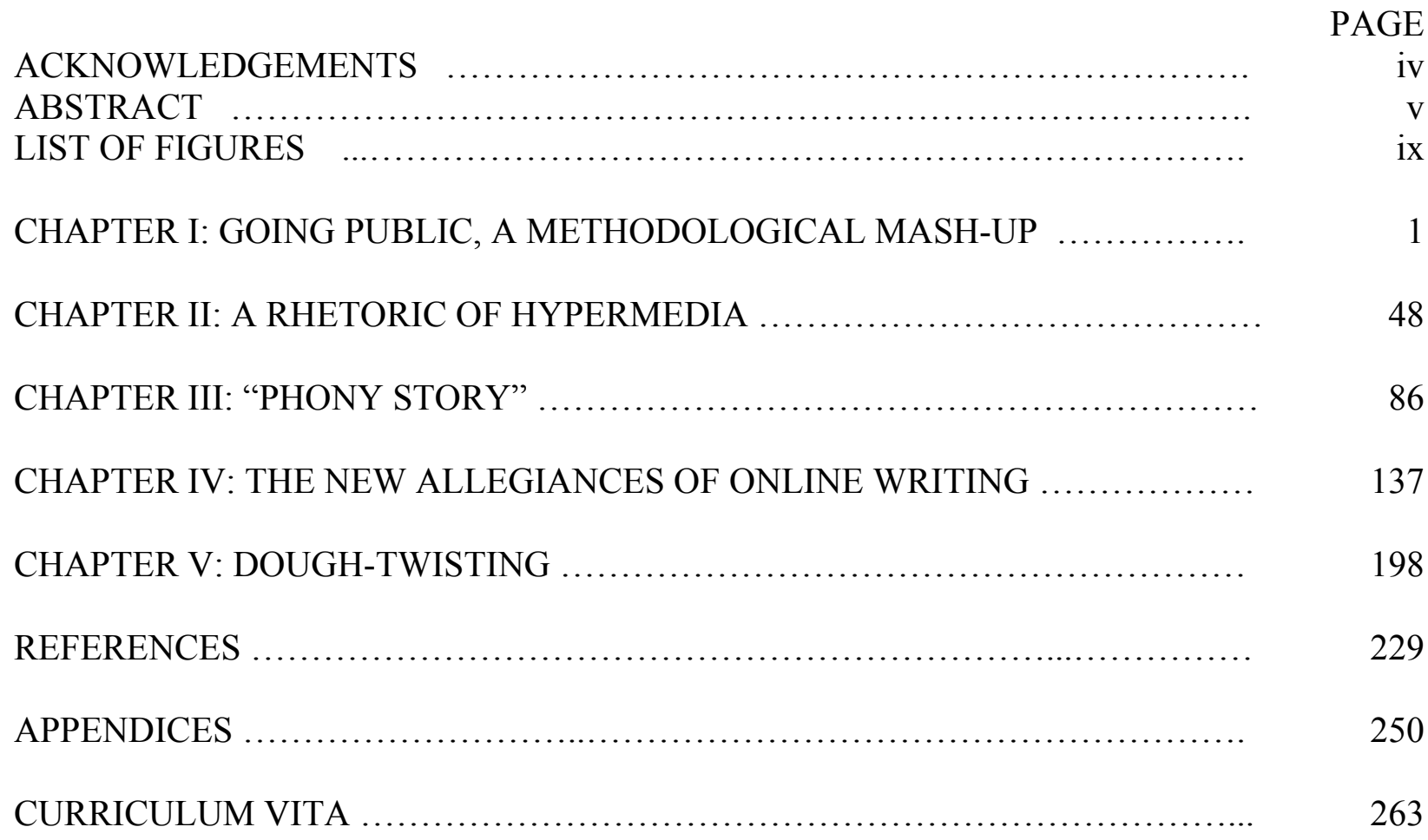




\section{LIST OF FIGURES}

FIGURE

PAGE

1. Figure 1.1: My citation-categorizing program—or "janky JSTOR."

2. Figure 2.1: Quantcast's estimated traffic for Newsweek and Slate from May 2, 2014 through April 29, 2015, in millions of unique users.

3. Figure 2.2: The majority of link citations on both Newsweek and Slate have anchor text that is three or fewer words long.

4. Figure 2.3: Average link count by section on Newsweek.

5. Figure 2.4: Higher link counts per article are associated with higher share, like, and comment counts.

6. Figure 2.5: The percentage of total articles that have a given number of links, in Slate and Newsweek.

7. Figure 2.6: A series of candlestick charts showing Facebook shares, likes, and comments for articles with particular pronouns in their headlines.

8. Figure 3.1: The percentage of articles per citation count, in $C E$ and $C C C$.

9. Figure 3.2: Never have citation strings accounted for more than $10 \%$ of all parenthetical citations in either $C C C$ or $C E$; the quadratic fit trend of citation string usage in $C E$ has remained fairly flat.

10. Figure 3.3: In every single case, both in $C E$ and $C C C$, the most-cited authors have a higher proportion of every type of citation strings and a lower proportion of parentheticals with just one citation. 
11. Figure 3.4: The percentage difference from the average proportion of most-cited authors at different citation count buckets.

12. Figure 3.5: The number of articles with a given citation count, in $C C C$ and $C E$.

13. Figure 3.6: In the twenty years between 1994 and 2014, in $C C C$ and $C E$, the number of articles using at least one eponymous adjective declined appreciablyif unsteadily.

14. Table 3.1: Eponymous adjectives in $C C C$ and $C E$. Mostly male, mostly dead, mostly Western, mostly outside of the discipline.

15. Figure 3.7: Number of articles that used the eponymous adjective "Bakhtinian" in $C C C$ and $C E, 1994$ - 2014, divided into categories of use.

16. Figure 4.1: Percent of articles in CCC using the word "website," "webtext," and/or "Internet," each year 1997 - 2014.

17. Figure 4.2: Percent of articles in $C C C$ including the name or URL of an online rhetoric and composition journal or to a website in CCC, 1997 - 2014.

18. Figure 5.1: Headline length, in words, charted against Facebook shares, likes, and comments. 


\section{CHAPTER I}

\section{GOING PUBLIC, A METHODOLOGICAL MASH-UP: \\ HOW I CAME TO CITATIONS, CODING, AND CALLING FOR A MORE-PUBLIC FUTURE}

I decided to pursue a $\mathrm{PhD}$ in rhetoric and composition because of a webtext:

Melanie Yergeau's (2009) "aut(hored)ism," a Computers and Composition Online piece about her embodied experience of being both a person with autism and an audienceaware author (an impossibility, according to researchers who believe autistics lack a “theory of mind"). The webtext's navigation menu was a silhouette of Yergeau's body, intersected by boxes that portioned out the text; thus, she demanded that readers participate in labeling her, putting her into boxes. Playful and thoughtful, visual and verbal, poetic and theoretical, the text seemed to me exactly what I imagined scholarship in the humanities should be: Speaking plainly, creatively, and compellingly to a potentially global Internet public about culture and language, with a goal of social transformation. At the bottom of the webtext's index page was the line, "Melanie Yergeau is a PhD student in English at Ohio State University." I Googled the Ohio State program, discovered that Yergeau was emphasizing in rhetoric and composition, and half an hour later was knee-deep in reading about rhetoric and composition doctoral programs. If this is what PhD students in rhetoric and composition are doing, I thought, sign me up. 
Before I began doctoral studies, however, in the year after finishing my Master's, I wrote full-time for a repair education and advocacy organization called iFixit. I co- and ghost-wrote op-eds with the CEO, maintained a site blog, and developed a series of articles about the social and environmental impact of electronics manufacturing and repair. In the process, I was offered a brief insight into online periodical publishing, when we submitted editorials to places including The Atlantic, Wired, and Business Insider (and received both feedback and line edits from editors). My writing changed rapidly: I got punchier. I learned to resist my academic tendency to equivocate. I began to read the news with an instrumental eye, looking for stories that offered a repair-oriented kairotic exigency for my own work. More schools introducing iPads for every child? Great, but there's no plan for fixing the iPads when they break. FitBit stock at an all-time high? Cool, but FitBits are so difficult to open that even the company would rather give you a new device than replace your dead battery or broken LCD. As I learned these new online writing practices, I became increasingly fascinated by the role of hyperlinks in argument. I began to develop a felt sense of when I should begin a new hyperlink. I learned how to gloss an ongoing conversation with a smattering of links. I got emails from people I had linked, thanking me for giving their work visibility. I also got emails from people begging for links, offering money or backlinks in return (offers, of course, that I did not accept).

As I prepared to return to academia, the iFixit CEO encouraged me not to go. One June afternoon at the end of a week in which we had published a Wired editorial, he pulled up site traffic data and showed me that nearly 200,000 people had read our article 
that week. Then, a pointed question: "How many people are going to read your dissertation, Elizabeth?" He correctly read in me a thrill at the audience, an excitement about being able to reach so many people. But I was looking forward to returning to the things I missed about academia: teaching writing, writing without always being "on message" (or at least somebody else's message), being able to talk about theory and expand beyond a tenth-grade vocabulary. Further, I believe that rhetoric and composition matters beyond academia: Anyone who sees advertisements or watches political speeches could benefit from a rhetorically analytical consciousness. Community organizers with meaningful causes could benefit from a primer of persuasion and guidelines of document design. I dream of a world in which strangers on the bus, hearing that I am a writing teacher, regale me with examples of how English is a living language, instead of preemptively apologizing for their poor grammar or lack of belletristic aspirations.

Thinking back to how much I enjoyed reading Yergeau's "aut(hored)ism," I imagined that, via webtext publication, writing academically and addressing a wider audience might not be mutually exclusive. Thus, not long after I started my $\mathrm{PhD}$ at the University of Louisville, I applied to join the staff at the open-access webtext journal Kairos - I interpreted its open-access branding and boasting of a "wide audience [...] hailing from Ascension Island to Zimbabwe" to mean that others there shared my goal. Kairos's "About" page began, "Kairos is a referred open-access online journal" and concluded with the claim that there have been "over 2,500 specific links to the journal and the webtexts that have been published in it"; I assumed that this interest in links and Ascension Island readers meant people there were also interested in reaching a broader, 
non-academic audience with rhetoric and composition research. That is, I read into the “About" page my own open-access proclivities and my increasing obsession with links. Certainly some Kairos webtexts support this notion: Claire Lauer's (2012) "What's in a name? The anatomy of defining new/multi/modal/digital/media texts," for instance, includes audio clips of such digital media heavy hitters as Cynthia Selfe and Gunther Kress discussing how the way we talk about writing limits our potential audience: Selfe said she talks about her work with a broader public "a lot," and when she does, she prefers to speak of videos, audio essays, and the like rather than using the term "multimodal composition"- - which is, she said, "a little bracketed term that we use within our discipline." Kress similarly described "wanting to talk to parents in schools or to politicians," and suggested that when a rhetorician speaks to such audiences, you "don't go through the kind of mire of attempting to educate them, but you go straight to what you want of them." Reading and listening to such webtexts, I felt the kind of academic excitement that comes from finding your scholarly niche- that eyes-wide hungry-reading excitement, that beatnik-poetry-slam-snapping excitement, that blinds-you-to-the-stuffthat-would-quell-your-excitement excitement.

If I had read more carefully, I might have noted that Lauer was writing about reaching a wider audience, but the text was not aimed at that wider audience- - which, precisely, of the 800 residents of Ascension Island wants to hear Gunther Kress hold forth on multimodality in an audio file embedded on a 2012 website that looks like it's from a decade earlier? I might have noted that Kairos's "About" page also said, "Kairos is one of the leading peer-reviewed journals in English Studies, made so by its dedication to academic quality through the journal's extensive peer-review and editorial production 
processes" [emphasis mine, beyond my poetry-slam-snapping phase]. If I'd engaged my more critical eye, I might have realized sooner that most webtexts are not aimed at a broader public. I might have considered who, indeed, would be inclined to seek out a peer-reviewed journal in rhetorical pedagogy. I might have found the Kairos style guide, which makes some fearsome demands of a hyperlink fan. But I read what I wanted to read.

It figures, then, that one of my first assignments as a Kairos assistant editor was Stage $3 b$, the "References" edit, which involves holding webtexts to the following requirements of the Kairos style guide:

All links should contribute to the possible meanings and readings of the texts. Linking for the sake of linking is discouraged (e.g., external links in-text to outside sources is usually discouraged in favor of links in the works cited; internal linking to the works cited is discouraged unless a text specifically requires it, and then back-navigation must also be provided). [...] Care should be given in linking to commercial sites in order to avoid promoting any particular companies or their products. If links must be made to commercial sites for the purposes of the webtext, they should be made to informational documents rather than sales pages when possible.

In fact, seven of the eight items under "Rhetorical Considerations" on the style guide discuss linking — mostly discouraging inappropriate linking. I went back through previous editors' work to determine the threshold between "contribut[ing] to the possible meanings" and "linking for the sake of linking." I saw links replaced with parenthetical citations, links removed entirely, links sent back to authors with the question, "Is this link really rhetorically necessary?" Given my conviction that links are important to the style and sharing potential of online writing, I found this discovery somewhat distressing. In my Kairos editing work since, I have set my own "rhetorically necessary" bar for hyperlinks lower than some other editors' bars appear to be, but I nevertheless feel as 
though I am betraying my own allegiances somewhat whenever I remove a link. As I entered the community of practice, I felt ill-equipped to make change, unsure how to make the argument I wanted to make about the importance of hyperlinks (grounded, as it was, mostly in speculation and a year of yet-uninterrogated personal experience).

I developed my research questions for this dissertation from that distress: Why do people cite the way they do, in academic and popular work, on- and offline, in hyperlinks and parentheses? Where do citation practices come from, and how do they reflect authors' intentions and allegiances? Are those citation practices connected to the circulation of work, and if so, how? What drives online writers and editors in rhetoric and composition webtext journals to write and cite the way they do? Could we help academic work circulate more widely online if we changed our online citation practices?

I began reading work in the field of citation studies: Researchers in English for Specific Purposes and other subfields of applied linguistics have conducted citation corpus analyses to understand the variety of academic citations (Swales, 1981, 1990, 1996), to analyze the differences in citation practices between disciplinary communities (Hewings, Lillis, and Vladirmirou, 2010; Hyland, 1999), and to analyze student writing (Thompson and Tribble, 2001). Meanwhile, I was learning how to program: I took a course in $\mathrm{C}$ and $\mathrm{C}++$, and I began to study JavaScript and jQuery. I decided that I would aim to answer questions about citations both in the abstract, via a hyperlink citation corpus analysis, and in the particular, via a series of discourse-based interviews with authors and editors about particular texts and their citations.

This dissertation is thus driven by two deceptively discrete foci: (1) how academics might reach wider public audiences, and (2) how and why people cite the way 
they do. I have, at times, found answers that address both questions at once. At other times, I focus more intently on one question or the other. In the interest of answering these questions, I also found other things that are just as interesting - and I do not wish to do a disservice to those findings by bull-headedly distorting them to fit my original conception of the project. Ultimately, though, each chapter will return to those questions and use the chapter's findings to attempt an answer to them, sometimes obliquely and always incompletely.

I have discovered that while many in rhetoric and composition share my goal of communicating our work to a broader public, precious little of our work makes its way beyond our disciplinary bounds. The most recent push to share our findings with "the public" can be traced back nearly 30 years, to social turn scholars including James Berlin (1988) and Patricia Bizzell (1990) who argued that the writing classroom is always already ideological, and thus we have a duty to address social issues. Peter Mortensen (1998) extended this public turn to suggest that we have an "obligation to air [our] work in the most expansive, inclusive forums possible" (182). In the aughts, Paula Mathieu (2005) identified and theorized an ongoing "public turn" in composition and rhetoric. We have long concerned ourselves with publics and counterpublics, in and outside of the classroom (for more, see Warner, 2002; Weisser, 2002; Edbauer, 2005; etc.). We have been successful, broadly, at several public initiatives: Thanks in part to Mathieu, community engagement work has gained significant traction in the field in the past few years. Yet we are still not communicating our work to the general public, even when our work would likely be interesting or useful. 
The need for reaching more-public audiences seems particularly profound in rhetoric and composition, which concerns itself primarily with two issues: (1) rhetorical and cultural analysis and (2) the teaching of writing. The first seems like it often would be of interest to the segment of culture analyzed, at the very least, and likely also to other audiences. Consider, for a small example, Paul Walker's (2016) article in the June issue of Rhetoric Review, which addressed the way writers for the mid-twentieth century British public anthropological research project Mass-Observation saw themselves as part of a rhetorical assemblage doing "good" for the future society, and how that sense of assemblage affected their descriptions of everyday life and trauma during World War II. Given the June withdrawal of the United Kingdom from the European Union, it seemed like a particularly kairotic time to be analyzing the written record of British nationalism. The world was very interested, in June 2016, in how British citizens understood their sense of individual duty to their nation, region, and world. Walker's article, however, runs as a parallel thread to the Brexit discussion, unavailable to the vast majority of the potentially interested public, unconnected to the worldwide discussion of Britishness. Given the glacial pace of academic publishing, there is no way an article like Walker's could have addressed the Brexit directly; ideally, however, the publication of his article at such an appropriate kairotic moment would have been paired with a more-popular research notes-style article connecting the findings to the fleeting international fascination with British nationalism. A publication aimed at publishing precisely that kind of research notes-style work is the praxical proposal and enactment I come to at the end of this dissertation. 
Similarly, though the teaching of writing has a smaller potential interested audience, writing teachers without access to composition research no doubt enormously outnumber the writing teachers with. We publish composition research mostly in journals that require expensive individual journal subscriptions and thus remain out of reach practically (if not completely) for most non-university writing teachers. Why? Because those are the journals we and our colleagues read, because publishing there is how we get our work recognized by the institution's tenure and promotion guidelines, because we distrust the alternative types of publication or doubt the reward of such publishing will be worth the effort, maybe sometimes simply because it is how it has always been done. I do not intend to trivialize such reasons; they reflect serious concerns that come from a deep vein of institutional practice. The risks, however, of not changing this practice are great: In a CCCC address over a decade ago, Doug Hesse (2005) expressed concern about the way public media has failed to pick up writing research in its discussion of writing: "For decades, the press, both popular and academic, has pundited why Johnny can't write and what Maria, his teacher, should do about it [...] CCCC didn't get that national press. What are these guys doing on our land?" (p. 341-43). If we continue to publish our work only in our own journals, we may remain irrelevant in the cultural conversations where our work would likely be of public interest, were it presented and distributed in an accessible form. We may inadvertently keep our writing and pedagogical research out of the hands of teachers who could put it into practice.

Online journals, which have proliferated in the last two decades, seem natural locations for writing aimed at the public. Yet although most online publications in rhetoric and composition are indeed open-access, most do not court a public audience in 
tone or practice - they are written by academics, for academics. Many of the 45,000 monthly international "readers" Kairos claims to have are likely lost in the site's $86.2 \%$ bounce rate ${ }^{1}$; the journal does not publish texts by non-academics, or even, generally, people outside of rhetoric and composition. Even Harlot of the Arts, which is explicitly aimed at a public audience, acknowledged in its most recent "Call for Solutions" (2016) that its attempts at courting the public have been unsuccessful:

Turns out our popularity among academics furthered the academic drift of submissions, which has led to a struggle to gain - and maintain — relevance for non-academic audiences. In this way, our "success" within the academic sphere has complicated the fundamental mission. We've attempted to expand our community - publishing pieces by state troopers, medical doctors, ceramists, lawyers, chaplains, film directors, web developers [...] Nevertheless, with a few notable exceptions, Harlot has not reached a wide readership among nonacademic audiences; we have also struggled to solicit submissions from outside academia, and outside rhetoric and composition specifically.

The editors go on to explain that, in its current form, the journal is no longer sustainable; its editors are overburdened and seeking help in redefining the journal or its mission. As of April 2016, the journal has paused in accepting submissions, and through May 2017 will accept only proposals for changing, redesigning, restructuring, or restaffing the journal. Why has Harlot been unsuccessful at reaching a wider audience? "If this stuff matters," the Harlot "Call” begins, "then why isn't it being shared?" This question is my key focus in the dissertation to come.

\footnotetext{
${ }^{1}$ Reported by WebStatsDomain. "Bounce rate" is the portion of viewers who find the site via a search engine and leave quickly after having visited just one page. Average bounce rates are around 60\%; a bounce rate over $70 \%$ is widely considered poor. Given that nearly every Kairos webtext is four or more pages, such a high bounce rate is especially concerning.
} 


\section{Perpetual Humanities Crisis}

Does “this stuff” matter? Kurt Spellmeyer (2012), citing rapidly declining English major enrollment rates and the infamously bad humanities $\mathrm{PhD}$ job market, argued that the first decade of the new millennium "has brought the humanities to their lowest point ${ }^{2}$ in a hundred years” (p. 567). Spellmeyer (2012) argued that the humanities' most important potential contributions to society are hermeneutical, offering context and coherence to a culture that tells entrepreneurial hero stories as if Bill Gates invented ragsto-riches. We must, Spellmeyer argued, demonstrate the continued relevance of the humanities by re-reading current political and social drama through the context-rich lens of literary history:

Where else except in the humanities can our students find the languages that will allow them to recognize, articulate, and act on a widely shared sense of discontent? Will they find those languages in marketing class? On television or in People magazine? None of these outlets has a memory, and therefore none is capable of preserving genuine alternatives to a status quo that seems less and less capable of keeping its promises. (p. 584)

Yet, as persuasive as I find Spellmeyer's argument for the continued importance of the "social imagination" we value so much in the humanities, I also find significant irony in the fact that it was published only in College English, only for other English professors. Who, among English professors, needs convincing of the value of hermeneutics? It is curious how Spellmeyer in one breath lauds the humanities for our "long-standing commitment to the public" (p. 582, citing Habermas and Benedict Anderson) and

\footnotetext{
${ }^{2}$ The humanities, we have apparently not tired of hearing, are in crisis. They have been in perpetual crisis since at least 1990, when Stuart Hall described how the socially transformative project of cultural criticism was at first reviled by most humanists, then eventually became toothlessly integrated into humanist inquiry. Or perhaps the crisis is, as Patrick Kavanagh (1999) suggested, the corporatization of the university and subsequent devaluing of professor labor — which, according to Kavanagh, we must fight by unionizing.
} 
suggests that in the humanities (as opposed to the falsely objectivist world of the sciences), "shareability is everything" (p. 583) — then in the next dismisses the likes of "television" and "People magazine." Shareability is everything, apparently, except when it comes to our own writing, which stays for the most part in paywalled print journals aimed at other academics. It is hard to imagine how we can continue to realize that public commitment when the public is, by and large, not exposed to significant university humanities education.

In the rest of this dissertation, I will take up and extend Peter Mortensen's (1998) answer to the crisis of the humanities: Literacy scholars within the humanities are ethically obligated, he claimed, to share what we have learned about literacy and writing with the general public. That public is being taught about literacy primarily by "journalists, essayists, polemicists, policy analysts, and others," and if we do not meet the public in those mass media fora, "we consign ourselves to mere spectatorship in national, regional — and, most importantly, local—struggles over what counts as literacy and who should have opportunities to attain it” (Mortensen, 1998, p. 183). Like Mortensen, I also argue that writing and rhetoric scholars must engage in international debate. That debate, however, has changed significantly and recently in location and tenor, since the rise of the Internet. To understand exactly how writing has changed - and to which values and allegiances we do, should not, and must continue to align ourselves - I conduct an investigation in three parts. 


\section{Maps: Of the Dissertation, of This Chapter}

In the dissertation that follows, I address my findings in a mixed-methods study of citation and writing practices in three different sites: In popular press web writing (on Slate and Newsweek-Chapter II), in traditional academic print text in rhetoric and composition (in CCC and College English-Chapter III), and in academic webtext online (on Kairos and Computers and Composition Online-Chapter IV). Chapter II conducts a rhetorical corpus analysis of Slate and Newsweek, seeking transcendent citation practices within each journal and considering how those practices (and other writing practices) and others correlate (or not) with social sharing; I then report on interviews with authors from Slate, aiming to elucidate those findings. Chapter III conducts a rhetorical corpus analysis of $C C C$ and College English, seeking an understanding of citation practices in the field of rhetoric and composition more traditionally, more historically; as in the previous chapter, these findings are commented upon and elucidated by authors/editors of each journal. Chapter IV considers hyperlink and parenthetical citation practices in webtext journals Kairos and Computers and Composition Online, via discourse-based interviews with several authors and editors for each journal. Chapter V draws parallels among my investigations and ultimately concludes with a proposal for a new kind of hytpertextual academic publication aimed at "the public"; it offers, at its close, some documents intended to sketch the shape of such a publication, including a "Rhetoric of Hypermedia" style guide for authors.

I want to take a brief moment to address the chosen citation style of this dissertation, with particular eye to why I have not included many hyperlinks: I argue that citations are rhetorical, that citation styles should reflect the expectations of the medium 
and genres of their location. I have thus chosen to use APA citations because they reflect the genre of the rhetoric and composition dissertation, and they also (more than MLA, because of their inclusion of years after authors' names) reflect the importance of the time of publication of all the sources I discuss; it is important to me, throughout this dissertation, that readers be kept aware of the comparative recentness of my sources. And while I do argue that academics should be writing more hypertextual argument, I want to be clear that this is not an argument for hypertext instead of but in addition to traditional academic text.

In the rest of this chapter, I will present an abbreviated history of rhetoric and composition's waning interest in hypertext, explain how I have come to see hyperlinks and citations as a representative practice, and then conclude with a fuller discussion of both my methodologies and my methods. That "methods talk" begins with a romp through my methodological underpinnings and ideological assumptions and ends with a chapter-by-chapter narrative of how I came to the particular methods I ended up using. Ultimately, I intend this chapter to address both the hows and the whys of my research: Why this textual practice, why these sites, why these methods and methodologies - and how it all came to be.

\section{Rhetoric and Composition's Smoldering, Moldering Love Affair with Hypertext}

Hypertext was originally proposed as a means of managing the increasing mountain of research that comes out of the modern university: Vannevar Bush (1945), in an article for The Atlantic, "As We May Think," described a machine that would change "our methods of transmitting and reviewing the results of research" by making 
connections between pieces of text. He described this device as the "memory extension," or "memex" for short, a tool that would enable "associative indexing, the basic idea of which is a provision whereby any item may be caused at will to select immediately and automatically another." Thus, it was intended to make intertextual connections explicit, to help us understand how ideas build upon one another and to make connections across disciplines. According to Jakob Nielsen's (1995) history of hypertext, in 1965, Ted Nelson coined the word "hypertext," and many other researchers over the course of the 1970s and 1980s (Andy van Dam, 1967; Doug Engelbart, 1968; Janet Walker, 1985; et alia) contributed to technological systems that resulted in the development of computerized hypertexts as we know them today. 1987 was a catalyzing year for hypertext, in a lot of regards: The international, interdisciplinary Hypertext' 87 conference brought together researchers from computer science and literature and biology and all the other fields that had heard inklings of a computerized system that promised to change the way all academics write and think.

Soon thereafter, it became fashionable among humanists to explore hypertextwriting tools like StorySpace and HyperCard, to imagine the authorial-intention-killing potential of what Espen Aarseth (1997) called ergodic literature, to read Michael Joyce (1996) — who often waxed poetic about what made hypertext such a radically new development in poetics and literature:

Truly interactive, constructive hypertext proposes choices in such a way that what happens in the viewing causes both the shaped form and the available choices to change. The interaction that hypertext aspires to is its own reshaping, an interaction some may see as its own overturning [...] Interactive art gives way to giving ways: Things could have been different $[\ldots]$ the becomingness of hypertext. (pp. 206-7) 
Joyce, with both critical and creative work demonstrating the potential of hypertext, kicked off an active decade of consideration: The fragmentary experience of reading hypertext. The relinquishing of authorial control. The ways texts can become deeply and visibly intertextual.

Any consideration of the intertextual, author-function-destabilizing potential of hypertext would be incomplete without a nod to George Landow (1992), who saw in early visions of hypertext the potential for the ultimate expression of contemporary critical theory. Landow connected hypertext to Ulmer's notion of a "morsel” (p. 9), Derrida's notion of an "assemblage" (p. 9), Bakhtin's notion of "polyphony" (p. 11), Borges's notion of an "Aleph" (p. 12), Bush's notion of the "memex" (p. 17)—and so on. While Landow's work makes some connections that excite me, especially as a fan of Derrida $^{3}$, the minutiae of early 1990s hypertext theory (and its links to contemporary critical theory) are beside the point of this dissertation.

Jay David Bolter (1991), in the hypertext criticism classic Writing Space, was a bit more circumspect about the potential for radical difference in hypertext's construction of the reader. Yet he emphasized the way that hypertext makes a reader aware of the process of reading:

\footnotetext{
${ }^{3}$ Such as, for example, the connections among Ulmer, Derrida, and hypertext: "In conversation with me," Landow (1992) said, "Ulmer mentioned that since Derrida's gram equals link, grammatology is the art and science of linking - the art and science, therefore, of hypertext. One may add that Derrida also describes dissemination as a description of hypertext: 'Along with an ordered extension of the concept of text, dissemination inscribes a different law governing effects of sense or reference (the interiority of the "thing," reality, objectivity, essentiality, existence, sensible or intelligible presence in general, etc.), a different relation between writing, in the metaphysical sense, and its "outside" (historical, political, economical, sexual, etc.)' (Dissemination, 42)" (p. 30). I am especially fascinated by the ways that hypertext theory positioned hypertext as the ultimate enactment of all the critical work language theorists had been doing for decades. In future research, I would love to explore the Joyce-Landow-Bolter vision of hypertext and consider what of that vision persists into modern single-page short-form networked hypertext.
} 
In following hypertextual links, the reader becomes conscious of the form or medium itself and of her interaction with it. [...] In its emphasis on process and on the reader's awareness of the medium, hypertext seems to belong to the literary tradition of modernism, and indeed modernist writers such as James Joyce can be regarded as forerunners of hypertextual writers such as Michael Joyce. (pp. 43-4)

Some take on these two visions of hypertext — as radical, destabilizing departure from literary tradition; or as less-radical-but-still-interesting self-conscious modernist-style dense and literarily meaty prose - drove the vast majority of literary hypertext, and of academic publishing about hypertext (much of which involved the same players, including Bolter, Joyce, and Shelley Jackson).

Kairos and Computers and Composition Online saw themselves, editors told me, as coming directly out of that tradition of literary hypertext. Cheryl Ball's MFA work was on literary hypertext, and, in our interview, she described that work as foundational to her early conceptualizations of the goals and mission of Kairos. In the second volume of Kairos, Doug Brent (1997) considered the role of hypertext in argumentation, broadly, suggesting that the question of whether or not argumentative hypertexts were really possible remained an open question:

Hypertext clearly has a lot of potential as a medium for information retrieval and for interactive fiction. But is it an effective medium for argument- what in the original sense of the term may be called 'rhetoric'? More specifically, what would be the effects both on readers and on writers if discursive argument migrated to a hypertext environment?

He suggests that rhetoric may, in its tendency to "privilege infinite hypotaxis rather than parataxis," be a poor medium for argumentation. The rest of the text goes onto consider questions such as "Is hypertext formless?" (not as much as people have said) and "If 'good' hypertext has no preset form, no default path for the reader, then has the rhetor any reliable way of presenting her thoughts to others?" (some control, maybe, but of 
course not total). He repeatedly expresses concern that it may be too early to tell whether hypertext can be rhetorical. Given the meta-discursive potential Joyce and Bolter highlighted in hypertext, it seems appropriate that one of the first online journals published an argument considering whether it is possible to argue in hypertext. Kairos, founded in 1996, was especially at the forefront of these explorations, founded by people including the electronic literature fan Mick Doherty and, soon thereafter, other literary hypertext proponents including Cheryl Ball.

In wondering whether or not hypertext could potentially argue, however, Brent was not alone. Lev Manovich (2001), in The Language of New Media, argued that the rise of hypertext was one of the many things killing rhetoric: "the sheer existence and popularity of hyperlinking exemplifies the continuing decline of the field of rhetoric in the modern era" (p. 77). Rather than considering the Internet to be like a giant library, he said, "it is perhaps more accurate to think of the new media culture as an infinite flat surface where individual texts are placed in no particular order" (p. 77). Hyperlinks, in their endless, rabbit-hole-like succession, signified for Manovich the death of purposeful arrangement and the rise of post-structural randomness. They modularized text, which he claimed makes purposeful arrangement impossible, which in turn makes rhetoric impossible. Links reduced rhetoric to metonymy, a data dump in the place of carefully constructed examples. He was not unequivocal in this pronouncement, admitting that "it is probably possible to invent a new rhetoric of hypermedia that will use hyperlinking not to distract the reader from the argument [...], but rather to further convince her of an argument's validity" (p. 77)—but at the time saw no such examples. 
Collin Brooke (2009) suggested that Manovich's claims - along with similar claims about the death of arrangement made by other hypertext theorists including Michael Joyce and Jay Bolter—were based upon "a straw version of arrangement" (p. 91). Brooke explained, "Just because there is more than one way to walk through a building, this does not make its arrangement (architecture) irrelevant" (p. 91). Nicholas Burbules (1998) similarly argued that hypertext has added to, not removed, modes of arrangement. "Bricolage and juxtaposition," as he named the two additional modes of arrangement, "imply just as much responsibility for selecting and ordering information in particular ways, inviting certain interpretations and excluding others" (p. 117-18). As hypertext has become less ergodic, less modular, and dramatically more common, it has become increasingly clear that hyperlinks do not make a text's arrangement irrelevant. Indeed, as people increasingly read heavily hyperlinked online arguments (from editorials to blogs to e-books to academic webtext), it becomes much more difficult to believe that hypertext cannot be the agent of rhetoric.

So many of Brent and Manovich's concerns seem no longer relevant—if fascinating - to a contemporary reader. Is it possible for a hypertext to argue? That question is about as settled as things ever get in the postpositivist world of the humanities. If the answer was "no," it would be hard to imagine Kairos could still existnot to mention the untold millions of online publications that make hyperlinked, networked argument. Yet, looking to the future, Brent suggested that the rhetoric of hypertext—while impossible to consider in appropriate depth in 1997—should be reconsidered at some unnamed point to come: "If hypertext does become a major medium for scholarly, argumentative, discursive, or philosophical rhetoric, we will 
therefore need to engage in rhetorical analysis of its rhetorical forms." We have not, I will argue below, done this to any great degree, and certainly not to the degree necessary to be using hypertext reflectively in our own practice and teaching it effectively in our classrooms. That is, rhetoric and composition has, disciplinarily, lost focus on hypertext; since that early glut of publishing, in the 1990s and early 2000s, in which researchers interested in writing devoted a lot of print text to hypertext.

Even the "reconsiderations" of hypertext (e.g. Johnson-Eilola's Nostalgic Angels, 1997; Bolter's second edition of Writing Space, 2001) are now old. Scott DeWitt and Kip Strasma's (1999) Contexts, Intertexts, and Hypertexts, for instance, considered hyperlinking in nodular, mostly-self-contained classroom hypertexts created in programs like HyperCard and StorySpace. Similarly, David Norton, Beverly Zimmermann, and Neil Lindeman's chapter in DeWitt and Strasma's book, "Developing Hyperphoric Grammar to Teach Collaborative Hypertext," described various purposes of links in student texts. Yet these hyperlinks were all internal—links to other pages within the same document, a style of hypertext that, while still extant in many websites, has fallen out of favor among argumentative hypertexts. Most arguments online are now single-page affairs.

The nature of hypertext has changed hugely, that is, since Johnson-Eilola, Bolter, DeWitt and Strasma, et al., were writing about it; it has become less ergodic, less exploratory, much more highly structured. Readers encounter hypertext so regularly that it hardly even registers as "hyper" anymore - it has become simply text. Many newspapers and magazines now publish two versions of the same text, one hypermediated (with links, with videos, with extra images sometimes in a slideshow, 
with comments and other metatextual elements) and one traditional print, delivered in (fewer and fewer) people's mailboxes or on their doorsteps. We treat those texts as if they're largely the same. As readers increasingly drop print subscriptions in favor of networked, on-screen reading, hyperlinks seem to fade deeper into the collective subconscious.

Nodding to Michael Joyce, Collin Brooke (2009) argued that we are in the age of "posthypertextuality" (xii), noting that "blogs and wikis, for example, are hypertexts, albeit in different ways than that first wave of thinkers conceived them" (xii). Yet, as Brooke pointed out, those hypertexts are built upon some of the same theoretical concepts that so compelled the hypertextual critics of the early- to mid-1990s:

Hypertext criticism carries some hints of the change that new media offers for the dynamics between writers and readers. However, because much of it is concerned with differentiating electronic discourse from print, those hints remain buried at the level of theory. For example, one of the most frequent analogies offered for the link is the footnote from print texts. Footnotes, of course, are subordinate to the main text; we might even describe them as subordinate to the main texts because they often contain references to other works. When we come across a footnote as readers, we either read it and return to the main text or we ignore it. Landow (1997) writes that "This kind of reading constitutes the basic experience and starting point of hypertext' (p. 4) and follows by asking his readers to imagine being able to follow the links in a footnote to other works as well. According to Landow, this scenario demonstrates the blurring of writer and reader, but it bears a strong resemblance to LeFevre's inclusion of the audience in the ecology of invention. (p. 78)

Yet because much of this hypertext theory originated in the hypertext (rather than the post-hypertext) era, it misses some of what is distinct about "post-hypertextual" hypertext. Single-page articles with external but no internal links do not destabilize the relationship between writer and reader in the same way writers like Landow saw in StorySpace-style hypertext. 
We have paid some attention to classroom uses of this "new" hypertext, especially, in the form of blogs. Over the last decade, classroom blogging exploded in popularity; it has perhaps passed its saturation point and entered a decline. In 2007, the word "blog" occurred 37 times in the CCCC program, including in 4 panel titles; through 2016, "blog” occurred between 15 and 28 times in each year's program $(2008,17 ; 2009$, $22 ; 2010,28 ; 2011,24 ; 2012,12 ; 2013,15 ; 2014,17 ; 2015,15)$; in 2016, however, "blog" occurred just 3 times - the lowest point in at least a decade. Blogging has become part of the background radiation of our discipline and our classroom practices. Though "hypertextual links" were named as a distinct feature of web writing in the 2004 CCCC position statement on "Teaching, Learning, and Assessing Writing in Digital Environments," the only interpretive mention of either the word "hyperlink" or "links" in the last 10 years of CCCC programs (that is, outside of front matter assuring readers that "links" to various things are available on the NCTE website) was my own 2015 presentation, which reported on the beginnings of this dissertation project. How can we have spent over a decade teaching and researching blogging with so little attention to hyperlinking, specifically, one of the most salient features of blog writing? I am baffled by the dearth of research on hyperlink practices and rhetoric; this dissertation seeks to make one foray into addressing that gap.

\section{Hyperlinks and Citations as Representative Textual Practice}

My original interest in issues of academic web publication focused on the practices of hyperlinks and citations: I started with questions about rhetorical velocity and the role of hyperlinks in the circulation of texts. My analysis suggests that links are 
indeed an important practice of web-aware writing. In my interviews, however, authors and editors offered me a much more comprehensive picture of the experience of learning to write and manage webtexts. In light of my interview findings, in light of the Harlot call, and in light of my own continuing interest in the potential public futures of webtext, I position citation (both hyperlink and traditional parenthetical) in this dissertation as a representative textual practice, one among many. In the 20 years since the webtext's birth, it has undergone rapid alteration - and paying attention to those changes, as well as to the values and practices that underlie citing and hyperlinking more generally, can help us chart a pragmatic and ideological course into a future of more-shared academic web writing.

Ultimately, I argue that to reach a wider public audience, we should be creating texts that are less concerned with unique visual design and more focused on publishing regular content with kairotic exigencies. Standard practices for webtext are still in development, and few webtext authors report having ever been given any guidance about how to write for the web; instead, they have been obliged to create their own internal style guides, for web writing practices including but not limited to citation. In developing these guides, authors draw on understandings of both academic print citation and popular press hyperlink citation. An ideal "rhetoric for the public" site would have a (somewhat) consistent "web writing practice"-based styleguide, designed to guide authors new to the genre, to create consistency across the site, and to increase the rhetorical velocity of the publication. 


\section{"Empirical Qualitative" Research: Combining Citation Corpus Analysis and Discourse-Based Interviews}

Because this project involves questions about texts at both a mass scale and at an

individual level, I selected a range of methods that helped me triangulate: Some are local, some are global; some are individual, some are institutional. I believe in mixed methods of textual analysis because we write, always, at the intersection of a range of intentions and goals, mandates and suggestions, histories and contexts. In the borrowed words of Mikhail Bakhtin (1982), “As a living, socio-ideological concrete thing, as heteroglot opinion, language, for the individual consciousness, lies on the borderline between oneself and the other" (p. 293). The author's "only power," according to Roland Barthes (1970), "is to mix writings, to counter the ones with the others, in such a way as never to rest on any one of them" (p. 146). We appropriate words from other people and fill them with our own meanings. Each word betrays the ever-negotiated tension between self and society. So to understand textual practices, we need to explore both authorial intention and on-the-page-or-screen reality. We must understand both how people think they are writing and how people actually write. This duality is especially important in understanding citation practices, where authors are always quite consciously borrowing words and ideas from other people; all language may be embedded and heteroglossic, but cited language is especially, explicitly so.

This specific value of my chosen quantitative method is over and above the value of mixed methods research more generally, which many researchers in our field recommend. Janice Lauer (1984), for instance, argued, “The use of several modes helps researchers to avoid a nearsightedness that overlooks many and sometimes even the most 
significant problems in a field because they exist outside the walls of a particular mode of inquiry” (p. 25). If I only conducted interviews and did rhetorical/discursive analysis, I might not be able to recognize the difference between typical and atypical usage of citations; if I only did corpus analysis, I would have to make big, troubling inferences about the hows and whys of citation practices. I consider myself what Bob Broad (2012) described as an "empirical-qualitative researcher," someone who "wants to work mainly with hard facts that are discursive" (204). I crave numbers and analyzable data-but only inasmuch as they tell me interesting things about people and the ways those people write and speak.

\section{Interviews Get at the Hows and Whys}

Considering authorial intention helped me illuminate a huge range of things not necessarily evident on the page or screen: For instance, without interviews, I never would have known that Slate author Rebecca Schuman and College English editor Kelly Ritter shared anxiety about being "dinged" for not citing enough — and both found that anxiety lessening over time, with experience; I never would have known that John Duffy did not place his own links in "Virtuous Arguments," or that all of Paul Muhlhauser's webtext co-authors have deferred to him in the question of proper link placement. In combining citation corpus analysis and discourse-based interviews, I am not alone: Ann Hewings, Theresa Lillis, and Dimitra Vladimirou (2010) conducted a citation analysis that combined a corpus-based approach, data/interviews from previous ethnographic inquiry, and a discursive analysis. In the introduction to their Journal of English for Academic Purposes report, they glossed a few of the ways people use citations: Citations "are a 
highly visible feature of academic knowledge," they help make "claims to a position within a disciplinary community," they reflect a "relationship between citer and citee," and authors may feel obligated to "[acknowledge] seminal works and the effect of the publication outlet" (p. 103). Few of the reasons why an author chooses to cite will be evident in the text itself; most of these things can only be revealed in interviews with authors.

Interviews also helped me to explore some of the socio-cultural dimensions of textual production; in Academic Writing in a Global Context (2010), Theresa Lillis and Mary Jane Curry introduced the concept of a "literacy broker," people who "mediate text production $[\ldots]$ with potentially significant consequences for how texts will be received and evaluated" (p. 87). In their interviews with authors, in an effort to shed light on the various social forces behind the production of text, Lillis and Curry asked their subjects to sketch the networks of people involved in the production of a given text (p. 63). These maps often included dozens of nodes, reflecting writers and editors and readers and friends and graduate students in various contexts and countries.

My interviewees similarly described complex histories of people involved in their citation and hyperlink practices. For instance, in my interviews for Chapter II, Ben Mathis-Lilley described how his BuzzFeed editor had encouraged him to place links over keywords to boost the site's Google rankings, and he explained that this recommendation continued to drive the choices he made when he linked. In my interviews for Chapter III, Kelly Ritter described how her editorial understanding of College English citations came from her interactions with Jean Gunner, who was the editor of College English when Ritter first published there; and from interactions with John Schilb, who was editor when 
Ritter took over. In my interviews for Chapter IV, Cate Blouke described how her decisions about how to cite Tweets in her Harlot piece "Pleased to Tweet You" came from the experiences of Tweeting during conferences, of asking attendees to live-Tweet a presentation she gave, and from talking with Paul Muhlhauser about the presentation afterward. These networks and histories, hidden behind and shot through citation practices, reveal the complexity of citation practices and helped me begin to get at the values and ideologies embedded within them.

\section{Corpora Paint a Bigger Picture}

Texts, of course, also function in ways that go beyond authorial intention. Even beyond the metaphysical, theoretical question of how much interpretation should depend on the author (e.g. Barthes, 1968), much of what I find interesting about citation practices happens below the surface, at the level of ideologies and unacknowledged tendenciesprecisely the sort of things that become evident en masse, in aggregate. Corpus analysis is uniquely positioned to bring to light otherwise tacit practices. Hewings, Lillis, and Vladimirou's (2010) citation corpus analysis, for example, sought "to see to what extent the geolinguistic context of publication affects citation practices" (103). Both Ken Hyland (2013) and Maggie Charles's (2006) citation corpus analyses examined reporting clauses in mixed-discipline corpora, comparing the types and frequency of different reporting verbs in different disciplines. Thompson and Tribble's (2001) citation corpus analysis compared citations from doctoral theses and undergraduate student writing, ultimately suggesting that in-class corporal activities might increase students' genre awareness. 
Corpus analysis allowed me to find patterns that authors did not recognize: in Chapter II, for example, I found that anchor text (the piece of text over which a link is placed) has a consistent and somewhat standardized length, and when authors stray from that length, they often do it with apparent (though sometimes tacit) rhetorical purpose. In Chapter III, I found that as people enter the academic community of practice in rhetoric and composition, their citation practices change (on average)—from citing more frequently, on average, to citing less frequently but with more citation strings and a higher likelihood of having excessively many or very few citations. In Chapter IV, I found that references to academic webtext have become increasingly common in our print journals (and references to "Internet" words have become the norm, rather than the exception), despite authors' persistent concern that citing web sources, even academic web sources, will make them seem theoretically light. Demonstrating these findings would not have been possible had I stuck solely to interviews.

\section{Together Is Better (Or, Why I'm an Empirical-Qualitative Researcher)}

Combining corpus analysis with interviews enabled a recursive, responsive methodology. Sometimes, authors' responses in interviews led me to look for particular things in my data—for instance, Kelly Ritter's expressed sense that newcomers cite "too much" led me to the investigation that makes up the bulk of Chapter III. Other times, I brought my findings to authors and asked for them to comment- - for instance, in my interviews for Chapter II, I asked Rebecca Schuman to comment on the finding that she cites more often than average for Slate (she attributed this to a sense that she need combat academics' complaints that her work is "unsourced"). 
Yet other times, authors' reactions helped me realize that I had made errors in my data collection or cleaning; when I first calculated the incidence of reference to web sources and academic webtexts in CCC and College English, I found a relatively flat distribution for both from 1996 - 2014. I shared the finding with Jonathan Alexander and asked, "Does that make sense?" His answer-which was increasingly emphatically negative, "It kind of doesn't. Huh. Does it make sense? No, because there's so much interesting stuff in Enculturation, even in Kairos"-prompted me to return to my data, where I discovered that my method had resulted in a huge number of false positives. In the "web sources" graph, I had included any text that had an apparent URL (e.g. included "http://" or "www."); this scored false positives for any article that referred to the NCTE $C C C$ or $C E$ pages. Similarly, in the "webtexts" graph, I had included a search for the word "Kairos"- though I only searched for texts that capitalized it as such, this still included any text that referred to the rhetorical meaning of the word kairos at the beginning of a sentence, again resulting in far too many false positives. When I accounted for these errors, the graphs I generated seemed to resonate much more with Jonathan Alexander's perception of the increasing prevalence and usefulness of web sources, academic and otherwise.

At the outset of my dissertation, I had little understanding of what I would find, but a clear conception that I wanted to use both quantitative analytics of some variety and qualitative analytics. I had hoped my ultimate output of the computerized analysis would be a comparative analysis of citations in my three sites - popular press, academic print, and academic webtext. But I had not really accounted in detail for how different those three sites are, and I only discovered through the research process how challenging it 
would be to get meaningful, direct metrics of comparison among sites. The number of citations per page, per article and so on are interesting - but only mean so much. The connections and comparisons I did find, however, were nevertheless interesting: Those comparisons had much more to do with the values, ideologies, and impulses behind citation practices.

I also came to a renewed, more nuanced appreciation of the value of mixedmethods research — and a new appreciation for its difficulty: My mixed methods did indeed give me insights that I would not have been able to achieve otherwise, and the different ways of looking at texts and authors and practices offered mutual insight. Yet I discovered that I discovered that quantitative and qualitative workflows are really different, and I could not prepare myself for the differences until I lived them. With Chapters II and III, I developed a mixed-methods workflow that looked something like this:

1. Come up with a quantitative analysis plan.

2. Collect the texts.

3. Write analysis programs to answer particular questions.

4. Generate graphs and other quantitative findings.

5. Share findings with authors, in interviews.

6. Go back to texts with understandings developed from interviews, and repeat steps 3 - 5 as necessary/desired.

When I decided to structure Chapter IV primarily via my interview data, without the same kind of massive corporal analysis effort, I discovered that I needed a new workflow entirely. Suddenly, I was no longer looking for patterns in texts and using an interview here or there to shed some very particularized light; I was looking for patterns in interviews, which required learning something about qualitative coding. I purchased Atlas.ti. I coded my interviews recursively (using what Johnny Saldaña [2016] calls 
"concept coding") and wrote dozens of messy pages, until I found conceptual connections — and then recoded using "pattern coding" (Saldaña, 2016). Thus, both my quantitative and qualitative workflows were recursive, involved returning to my data and then back again to my text—but they required different attitudes, different approaches, and different ways of looking at research itself.

Thus, I discovered some deep resonance between my goals in embarking on this mixed-methods project and the methodological experience of it: Each new method challenged my assumptions about research, about writing, about data. My methods turned back on themselves and on each other. I found myself embroiled in what Rebecca Rickly (2007) has called the "messy contexts" of methodologies, in which research evidences itself "not as an ordered, neat, linear procedure, but one that is integrated, messy, and non-hierarchical" (p. 9). Pieces of my research declined to stay put, atomically vibrating like Rickly described, demanding renewed attention when combined with the rest. In the three narratives below, I trace how the methods for each chapter grew out of my initial methodologies - and how my resulting methodology was shaped by that experience. I describe how, in Chapter II (Slate and Newsweek), I learned how to write a web crawler and query Facebook for data about how often particular articles have been shared, and how learning what I could do (and what was beyond my current abilities or available time) shaped the questions of my research. I then describe how, in Chapter III (CCC and College English), I discovered that I would need to clean and categorize the data I collected to a greater extent than I had imagined — and how that realization limited the kinds of questions I could ask, but also inspired me to ask questions about practices such as eponymization, which are visible via distant reading without more categorizing. 
Finally, I report how, in Chapter IV, I developed a method of snowball sampling, coded my interviews, and came to the eventual understanding I did. In these narratives, I hope to shed some light on the messy contexts of mixed methods research.

\section{Methods, Chapter II: Finding Patterns in 8,000 Slate/Newsweek Texts}

My popular press chapter began with the desire to learn about practices in webnative publications. Once I had that research question-how do web journalists cite?-I needed a method to answer it. In short, I wrote a series of JavaScript/jQuery programs, using Node modules downloaded via Node Package Manager (including Crawler ${ }^{4}$, Cheerio $^{5}$, Request ${ }^{6}$, and XML2JS ${ }^{7}$ ), that pulled about 4,000 articles each from Slate and Newsweek, stored that data in a MongoDB database, and then calculated some basic statistics about the articles' citations. For more, see Appendix E, a flow diagram of my Slate-crawling program. Although a programmer would likely find that explanation sufficient (combined, perhaps, with a link to my GitHub profile, ${ }^{8}$ where you can see my code itself), I suspect it will be rather meaningless to most compositionists; two years ago, it would have been meaningless to me.

Meandering and Recursive: Some Suggestions for Graduate Education

There have been many calls (e.g. Burdick, Drucker, Lunenfield, Presner, Schnapp, 2012; Hurley, 2010; Yancey, 2009) in our field for graduate education that enables students to develop technoliteracies and create tools for Internet research. I

\footnotetext{
${ }^{4}$ https://github.com/bda-research/node-crawler

${ }^{5} \mathrm{https} / / /$ github.com/cheeriojs/cheerio

${ }^{6} \mathrm{https}: / /$ github.com/request/request

${ }^{7} \mathrm{https} / / /$ github.com/Leonidas-from-XIV/node-xml2js

${ }^{8}$ https://github.com/elizzybeth
} 
cannot say that my method of learning is fully replicable on a disciplinary scale, given how much I was helped by having a partner who is a professional programmer; when I found myself beating my head against a wall, I could ask him to help me find the sledgehammer. I was aided also by other fortunate events: A non-teaching fellowship allowed me the time to take a beginning programming course in the first year of my doctoral program and return to my coding self-education in my fourth year. My enthusiastic mentor, Dr. Mary P. Sheridan, encouraged me to push disciplinary boundaries, connecting me with a big data computer science researcher on campus (Dr. Olfa Nasraoui) and remaining remarkably sanguine when I had to confess repeatedly, "This is taking longer than I expected." My experience suggests how meandering and recursive the development of Internet research tools can be - supporting Kathleen Yancey's (2009) argument that digital graduate education must be flexible enough to respond to the changing face of technology. It suggests, too, how a combination of formal courses and directed self-teaching can result in functional Internet research skills. Hence, below, I offer a narrative as to how I developed this method, from my novice position, in hopes that it might make the work replicable for anyone wishing to embark on a similar project and/or develop graduate education that enables students to do this kind of research.

I also believe that several of the tools I used in developing my methodology might be useful even to Internet researchers who are not comfortable with coding: The Facebook Automated Programmer Interface (API), for example, makes it possible to get counts of times a particular link has been liked/shared on Facebook. I was making calls to the API automatically, but it is possible (albeit much slower and clunkier) to use this 
functionality even without any coding. The graphing tool I used, plot.ly, also has many functions available from within a web browser with no coding, though I accessed it via a Node JavaScript module; compared to Excel, plot.ly has a wider variety of graph types and its graphs are much more customizable_-plus, it has detailed tutorials that explain the math and logic behind its various graph types.

\section{Crawl before You Write: Developing Web Crawlers}

As I mused on how I might chart citation practices online, I began to discuss the idea of a hyperlink-categorizing program with programmer friends: At first, I imagined a program that would go through a list of articles and bring up the links, one by one, for my own hand-coding (e.g. What kind of site is this? How long is the linking phrase? Where in the article does this link appear?). I knew enough $\mathrm{C}$ from my introductory programming class that I felt confident I could do the "CRUD" side of the program: create a record for each article, read through the article to find links, update the categorization of each link, delete errors. But all my web programming experience to date was front-end: I could write a simple website with HTML and CSS, but I could not write a program to read one. My friends told me I needed an automated method of reading through websites and loading their links, commonly called a web crawler, the same sort of program that makes it possible for Google to find and categorize pages. $\mathrm{C}$, my partner said, was the wrong language, not designed for working with the web - and why would I bother hand-categorizing when I could make the program do it for me? He instead recommended jQuery, a JavaScript library that turns an HTML website into a hierarchical model, making it easier to read through en masse. 
Armed with that scrap of information, I embarked on some haphazard research. I read the Wikipedia page about web crawlers, took some free online courses in JavaScript and jQuery, and started reading tutorials. Eventually, I came upon a bare bones web crawler $^{9}$ that I could implement in my program: It could request a website from a server and bring back information from the site. When I had installed the crawler and modified my own program to fit the specifics of Slate and Newsweek, I could feed it the URL for the site's main archive page, and it would automatically crawl through each of the articles within. By reading through the source code from each of my target sites, I identified the elements I wanted to save: for instance, the author's name appears on each Slate page wrapped in an $<\mathrm{a}>\operatorname{tag}$ (indicating a link) with the ID "main_byline."

I then had to save the data I collected somehow. At the suggestion again of my programmer partner, I selected an open-source database system called MongoDB, which I again explored through a series of tutorials and examples. For each article, I chose first to save the full text, HTML, URL, author names, title, section name, publication date, and retrieval date - in addition to separate entries for each of the links within. Each link was stored with its anchor text (the word or words that point to the linked page, historically rendered with a blue underline), its linked URL, the length of its anchor text, and the word count of its anchor text. After collection, I ran a series of analysis programs: One searched for and categorized links that pointed to other articles on the same site and on sites owned by the same parent company, and also calculated averages (anchor text

\footnotetext{
${ }^{9}$ In a node.js package, a piece of code made available by other programmers for easy JavaScript implementation. See Appendix C for the code of my Slate web crawling program.
} 
length, links per word and per paragraph, etc.). ${ }^{10}$ This analysis gave me answers to some of the most basic questions about citation practices: e.g. how often articles linked per word and per paragraph, how long anchor text was, on average. Another analysis program used those averages to consider whether links per word correlated at all with social sharing (using the data from the Facebook API); yet another divided the corpus by author, so I could compare my interviewees' hyperlink practices with practices of other authors.

I wrote the original version of the Slate crawler program in March 2014, to present at CCCC. I knew soon thereafter that I wanted to send my crawler to catalogue another site, and I eventually settled on Newsweek, for its interesting history of moving in and out of print. Newsweek offered an example of a publication that was very clearly trying to change its online image to better match the cultural zeitgeist. If Slate is webnative, Newsweek is web-newcomer-but not new at all to journalism more broadly. Newsweek also offered an example of another kind of Internet monetization: Slate is monetized through advertisements, whereas Newsweek has a paywall. Since I am interested in the capital exchange of link systems (both economic and cultural capital), I thought it might be useful to examine a different system. ${ }^{11}$

I did not predict, however, how much more difficult the paywall would make adapting the crawler to the site. After many failed attempts at crawling, I discovered I had

\footnotetext{
10 These averages

${ }^{11}$ Differences between the two sites that might be attributable to their paywalled vs. free structures did not end up being a significant variable of my analysis; it is, however, one that I would very much like to pick up in future research.
} 
to give the crawler a cookie from a logged-in session, ${ }^{12}$ which essentially allowed it to masquerade as me; after it crawled a few hundred pages, I would have to give it a new cookie. Even then, I found myself running up against site rate limits — at about 4,000 articles, the crawler would hit an unmovable paywall that seemed to last a couple of days, no matter how freshly baked its cookie. These limits made iterative development a slow and often frustrating process. When I was getting bad data, I was often not sure whether it was a fault of my program or the limits.

\section{“They Really Like Me”: Facebook API Share Data}

I realized that the data I had collected would not allow me to make any claims about the circulation/distribution of the articles I was examining, and so I turned to the largest publicly available record of social sharing data: the Facebook API. By feeding URLs to the API's share data feature, I could get counts for how many times each article had been shared, liked, and commented upon on Facebook. This data could suggest, better than any other metric I can access, how much an article has been picked up by the world at large. By comparing Facebook data to my other metrics, I could identify preliminary correlations between citation practices and circulation.

\section{Talk about It: Discourse-Based Interviews}

Once I had data, I conducted interviews with two Slate authors/editors (I also contacted some Newsweek authors but did not hear back) who agreed to speak with me about their citation practices: Rebecca Schuman and Ben Mathis-Lilley. We spoke

\footnotetext{
${ }^{12}$ Once I learned how to falsify this cookie, it was not complicated: At the beginning of my crawler callback function, I added a single line of code that began, "headers: \{ 'Cookie':" and then included a copypasted line I'd taken from watching data sent by my browser to the site,
} 
generally about their histories and experiences in journalism more broadly, and then I asked them to pick an article they had written recently and describe that article's "citation story" - why they chose to cite the things they did, what led them to put links over particular words, what kinds of citation-related editing (if any) their own editors have conducted, and so on. By focalizing discussion on a particular piece, we were able to get into some detail about the rhetorical decisions behind individual citations. I also reported my data on both authors' citation practices, compared to the site-wide average, and asked them how they might account for the differences.

\section{Methods, Chapter III: Making My “Janky JSTOR”}

I began the investigation of my third chapter, as always, with questions: How do we cite? How do we talk about citation? How do actual citation practices align/differ from the way we teach citation? How can citations help explain, define, or reveal disciplinary trends? This chapter builds on Derek Mueller's (2012) analysis of citations in $C C C$, both in theory and in method. Like Mueller, I seek to construct telling narratives about the shape of rhetoric and composition over the last twenty years, through citation analysis and graphs. The stories we tell about the shape of our field often begin and end with narrative: metaphors of writing; models of writing processes; narrative as subject, method, and analytic lens. Given that we are storytellers and teachers of storytelling, this predisposition toward disciplinary stories makes much sense. As Susan Miller (1993) put it in the opening of Textual Carnivals, "power is, at its roots, telling our own stories" (p. 1). I do not wish to take up arms in what Richard Haswell (2005) termed the "war on scholarship," in which quantitative and qualitative methods are pitted against one another 
to the detriment of all; instead, I see graphs and narrative as complementary forms, as different ways of telling stories about data, texts, and people. Mueller explained his own championing of graphs thus:

1) deliberately altering scale allows us to see aggregate patterns linking details and non-obvious phenomena, and

2) the systematic compilation of replicable data may empirically corroborate local, tacitly felt impressions about changing disciplinary conditions (p. 196)

That is, whether graphs seem to confirm the stories we've told about our field or point toward new truths, they enable different kinds of understandings than those we get through storytelling, which is limited by tropes and monomythic tendencies. Graphs make the tacit explicit, and they can draw out hidden stories. Ultimatelyrome, graphs are in some ways a highly narrative form: they give data a story, which is elaborated upon in analysis and discussion. I am also building on Mueller's work much more literally, in that I have borrowed his list of the 110 most-frequently cited authors in $C C C$ from 1987 to $2011,{ }^{13}$ as a means of indicating disciplinary centrality.

\section{Corpus, Cleaning, and Categorizing}

My corpus consists of 1,327 articles, collected from CCC (1997 - 2014) and College English (1994 - 2015, not including 2007 and $\left.2008^{14}\right)$. I wrote a program in jQuery that, with a web crawler, accessed the University of Louisville ProQuest archives

\footnotetext{
${ }^{13}$ Because I use these names to search for authors of articles rather than authors cited in those articles, I have eliminated four names from the list that I knew were not authors of any piece in my corpus: "CCCC," "United States," "Aristotle," and "bell hooks." Thus, my "most-cited" list is made up of the other 106 names on Mueller's p. 202-03.

${ }^{14}$ Of databases that subscribe to CCC and College English at UofL, only ProQuest has HTML full textand ProQuest, unfortunately, does not have all back issues. Though 2007 and 2008 College English articles are available via ProQuest, my crawler was unable to pull data from those years. I did not realize until far too late in data analysis that those years were missing in my corpus; to add them to the database, I would have to crawl, clean, categorize, and analyze the whole College English corpus again from the beginning, which proved prohibitively time-consuming for this project. A task for a future analysis, perhaps.
} 
of $C C C$ and College English, downloaded the full text ${ }^{15}$ of all available articles, and saved that text alongside some metadata (e.g. author, title, publication date) to enable later sorting and searching by those elements. When I began to pore over my collected data, it became clear that I would want to exclude types of articles that typically do not include scholarly research: editorial comments, errata, response essays, and book reviews. Both $C C C$ and $C E$ identify these articles with a phrase preceding the title. Using a regular expression search (a pattern-matching sequence), I removed from the corpus any article with a title beginning with a series of phrases. ${ }^{16}$

I then attached to each article's entry in my database a list of every parenthetical statement in that article, along with an automated count of how many works were cited in that parenthetical, and an automated guess at the type of citation. The automated counter simply searched the parenthetical for any semicolons and added one; the automated typeguesser checked the parenthetical against a series of regular expression patterns (e.g. any word and then a number was categorized as an author's name with a page number; just a number was categorized as a page number; anything with semi-colons was categorized as a citation string). As I worked, I tested the automated counter against one particular citation-heavy article: Karen Kopelson's (2015) CE piece about workplace guides for adults with high-functioning autism, which conveniently provided examples of every type of citation I was hoping to automatically categorize. I was able to fairly consistently categorize parentheticals that included nothing but an author's last name and a page

\footnotetext{
${ }^{15}$ I experimented with downloading PDFs and using several different kinds of Optical Character Recognition (OCR) software for static text, as Mueller did, but even paid OCR software was highly errorprone. For the volume and specificity of my desired analysis, I deemed it inadequate.

${ }^{16}$ Articles with titles beginning with these phrases (using a case-insensitive regular expression search) were excluded from the corpus: "Anon: Announcements and Calls"; "Anon: CCCC News"; "Anon: Erratum"; "Anon: Error"; "Comment:"; "Comment \& response"; "Comment \&amp; response"; "ERRATUM"; "From the Editor"; "RESPONSE"; "Review:"; "review of"
} 
number. I quickly discovered, however, that it would be essentially impossible to distinguish automatically between a parenthetical that was simply an aside and a parenthetical in which an author made an aside and also cited another author's name. Other thorny problems — such as distinguishing between a year in parentheses and a fourdigit page number in parentheses—-defied my automatic categorization. Thus, I decided to write a program that would bring up each parenthetical one by one in a web browser for human confirmation/editing (see Figure 1.1).

This program (which I took to calling my “janky JSTOR,” because it essentially provided a crude way to read through the whole journal) listed the titles of all articles in my corpus on the left. When an individual article title was clicked, the article's full text came up in the window on the right, with the first parenthetical statement in the article highlighted. A box below the text had the automatic citation count. The human categorizer could confirm that count by hitting "enter," or could change it and then hit "enter" to confirm. I categorized the first half of the $C E$ citations; after that, I requested the help of two undergraduate research assistants, who assisted me in completing the rest of the categorizing (see Appendix A for the protocol and frequently asked questions list provided to those assistants). 


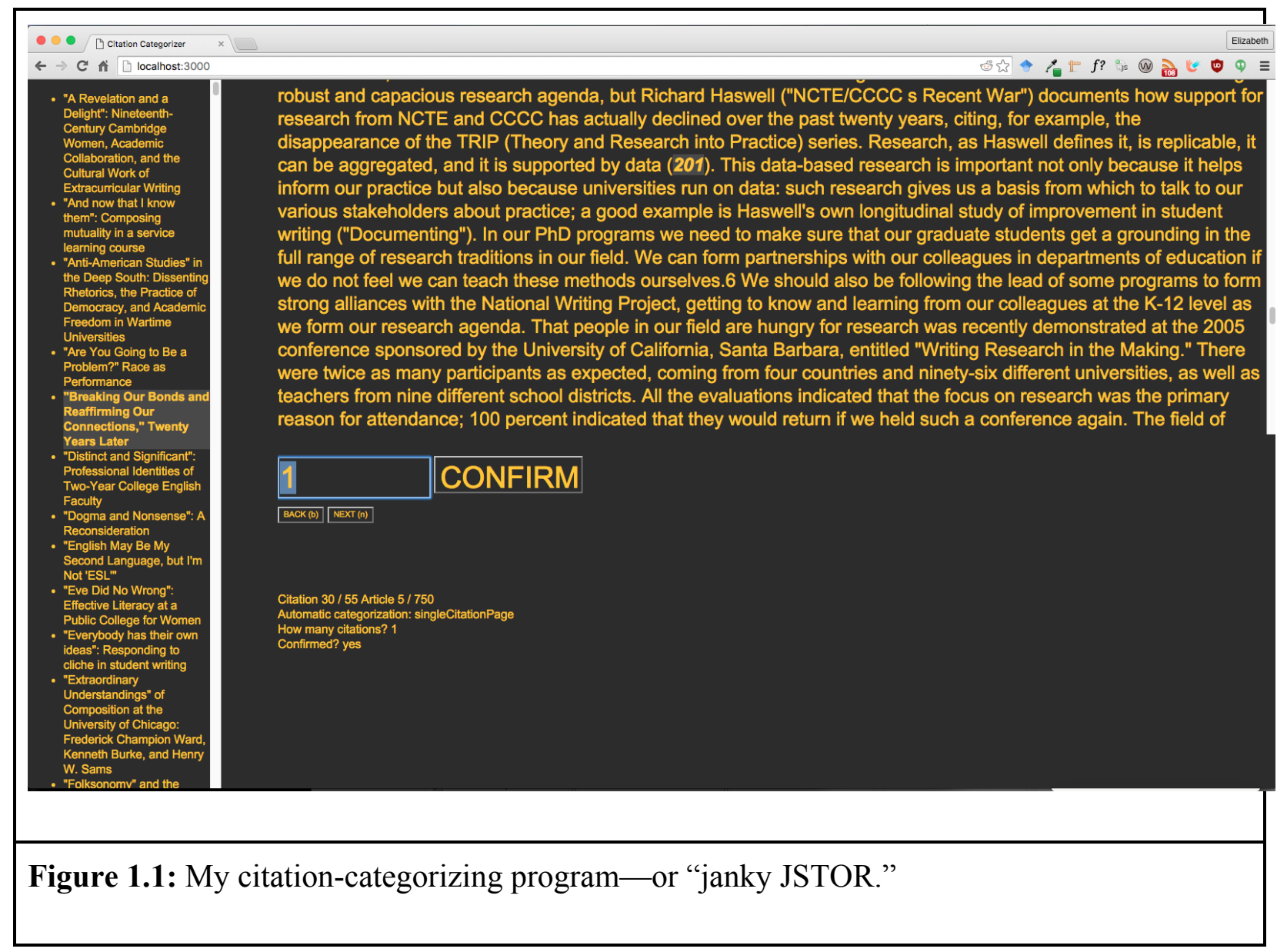

I then wrote a series of JavaScript programs to analyze the data, looking for distributions

of citation counts by various groups of authors (Derek Mueller's “most-cited” list, authors who have only published once in a given journal, and $C E$ 's "Emerging Voices" featured authors). Some of my other analysis programs look at incidence of citation strings, frequency of reference to Internet addresses, and proportional use of eponymous adjectives. In all, I hope to paint a picture of citation practices in our discipline's flagship journals in the last 20 years, with the goal of finding union and disunion with the stories we tell about our field. 
To deepen my understanding of this picture, I have also conducted interviews with the current editors of $C C C$ and $C E$, Jonathan Alexander and Kelly Ritter. I have built my investigation of disciplinary narratives from those interviews. Several times, my interviews would suggest a line of investigation, I would turn to my database and explore that line, only to discover that my data seemed to conflict with what my interviewees said. This recurrent experience of disjunction brought me to the notion of aporias, the idea that more interesting than practices is the way that practices often work against disciplinary values

\section{Methods, Chapter IV: Learning to Do Qualitative Research}

I began my final data chapter with broad, exploratory interviews - in fact, the first interviews I conducted for the dissertation in whole - in early spring 2015, with Doug Eyman at CCCC, Kris Blair at Computers and Writing, and Cheryl Ball via Skype. I initially envisioned that, as with previous chapters, these interviews would be paired with a bigger citation corpus analysis of Kairos and Computers and Composition Online $(C \& C O)$. As I wrote the programs for my other chapters, however, it became increasingly clear to me that doing a large-scale corpus analysis of webtextual journals would be quite challenging, if not practically infeasible. I was able to collect so many texts for analysis in my previous chapters because every text in each of those publications is structured in essentially the same way - this consistency makes it possible to write a web program that iterates over each one and collects data from it. Because every webtext has a different structure, a jQuery program could not effectively iterate over each one. Furthermore, once I began conducting interviews with authors and editors, I found that I was 
discovering such rich data about webtextual practices that I would be hard-pressed to find time and space in one chapter to address both the qualitative data and a separate quantitative analysis. As someone who has written for $C \& C O$ and edits for Kairos, I have some previous insight into publishing practices at both places, and my dense interview data resonated with and at times challenged my previous understandings. I also discovered that several of my quantitative questions about webtext practices - how often are people in the field citing webtexts? how much do titles affect social sharing?-I could answer with my previous Slate/Newsweek and CCC/CE datasets.

To select authors, I used small-scale "snowball sampling": I asked Ball, Eyman, and Blair to point me in the direction of texts they thought had particularly interesting "citation stories." Ball and Blair named several webtext authors from each journal's most recent issues; I successfully contacted most of the authors they named. I also picked one author from Kairos whose text I had worked on as an Assistant Editor and whose Chronicle column I read, Anastasia Salter; and one author who I had been told by several people would likely have valuable insights into my questions about public intellectuals, John Duffy. Ultimately, I conducted hour-long interviews with these eight authors and three editors, asking some discourse-based questions and some more-open answer questions; see Appendix B for a representative list of questions I provided webtext authors Dawn Formo and Kimberly Neary before we talked. Most authors who had worked as collaborators, I was able to interview at the same time (e.g. Muhlhauser and Kachur, Muhlhauser and Schafer, Formo and Neary), via video chat. Cate Blouke, who had collaborated with Paul Muhlhauser and Daniel Schafer on May the \#Kairos Be With 
You, I had to interview separately due to time constraints—-but we also discussed some other webtexts she had written.

After transcribing the interviews with Express Scribe and a foot pedal (which resulted in 134 single-spaced pages of interview transcripts), I conducted two primary cycles of coding in the qualitative research software Atlas.it. In the first cycle of coding, I went through two interviews (Blouke's, and Mulhauser and Schafer's) to develop a short list of first-impression codes; Johnny Saldaña (2016) called the method I used "concept coding," including "a search for the properties and dimensions of categories—conceptual ideas that bring together similarly coded and related passages of data" (p. 118). My second cycle of coding, conducted immediately following, was recursive: Whenever I added a new code, I would skim back over the earlier interview transcripts looking for places that might match the code. In both cycles, I used what Johnny Saldaña (2012) has called "Simultaneous Coding," often labeling sections with two or more overlapping, intersecting codes.

Ultimately, I ended up with a list of 55 codes, 44 of which I used more than once. Of those 44, the top five were "affordances" (22 uses), "genres" (21 uses), "public audience" (19 uses), "citing online works" (16 uses), and "classroom" (15 uses). Then, in a new document, I collated responses for the tags that seemed most interesting or telling, in terms of webtext practices, writing for a public audience, and hypertext citation. From that list, I began writing: I wrote several pages about each of the top five affordances. At the time, I imagined these pages were the meat of the dissertation chapter-but I now think they might be better described as "analytic memos" (Saldaña, 2016, p. 44). In them, I began to make connections among my codes. And eventually, after several months of 
trying to make sense of the categories I had used, I came to recognize a new pattern among them - a pattern I began to term "allegiances," the new kinds of assumptions and understandings that authors betrayed in their discussions of what mattered when they wrote online.

\section{The End of the Beginning: Recursion, Methods, and Concluding This Introduction}

I have intended in this introductory chapter to provide a series of narratives that situate my research — personally, disciplinarily, and publicly. My goal has been to explain how and why I came to each aspect of my investigation, from my entry into the field as a whole through my choice of particular computer coding languages and qualitative coding systems. These narratives, I hope, offer a roadmap for future students seeking to conduct similar kinds of mixed-methods rhetoric and composition research; I turned to the dissertations of my friends and colleagues as I made decisions about methods and methodologies.

I imagine, too, a potential for disciplinary change, for courses and programs to adopt practices that enable mixed-methods research. For instance, programs might provide opportunities for graduate students to take courses in computer languages, or perhaps even invite computer science professors to teach or co-teach courses in text mining and humanities-focused data analysis. Such support would have likely enriched my investigation — and might make such an investigation possible even for students who, unlike me, do not have a professional programmer partner whose brain they can pick upon getting stuck. 
Finally, I have gestured in this chapter to the findings and conclusions of the rest of this dissertation: There are significant, demonstrable consistencies in the practices of both hyperlink and print citations. In those practices, we can read values—of genres, of disciplines, of medium and mode. And by investigating those values, we can begin to understand in greater detail some of the qualities that make web writing different from what has come before.

Ultimately, I call for a new kind of web writing publication online, a secondary research publication, that disseminates "research notes" versions of writing researchdesigned for public distribution, connected to kairotic moments, and aimed at a wide readership. This dissertation concludes with a detailed, pragmatic proposal for such a publication. I hope — indeed, I expect — that my future in this discipline will involve creating venues for the kind of work that so excited me when I first encountered Melanie Yergeau's “aut(hored)ism": Places where writing researchers are encouraged to speak plainly, creatively, and compellingly to a potentially global Internet public about culture and language, with a goal of social transformation. 


\section{CHAPTER II}

\section{A RHETORIC OF HYPERMEDIA: LINK CITATION PRACTICES AND WEB JOURNALISM}

The Internet has become perhaps the most potent forum for modern public debate. Print journalism seems in peril, as readers increasingly consume news delivered in social media and aggregation feeds, rather than on their doorsteps. Instead of passively receiving this news, readers often engage, by commenting, liking, and sharing. Franklin Delano Roosevelt was praised for the directness of his "fireside chat" radio addresses; Barack Obama made similar headlines in 2012 for participating in a question-and-answer session on social news aggregator Reddit's “Ask Me Anything” forum. People could, of course, speak back to media before the Internet, but never as quickly, easily, or with as little censorship. These changes in literacy practices, as Andrea Lunsford (2013) has argued in an op-ed, "alter the very grounds of literacy as the definition, nature, and scope of writing are all shifting away from the consumption of discourse to its production across a wide range of genre and media" - yet, she said, we do modern youth a disservice if we assume that their changing literacy practices mean they are less literate.

Lunsford's op-ed, however, epitomizes the problem I aim to address in this dissertation: though written for a non-academic audience to combat the public perception that digital literacy is an oxymoron, it was published only as a static PDF document on the Stanford Study of Writing website and as an entry in a first-year composition reader, neither of which is a medium nor a forum designed for wide-scale public sharing. As far 
as the connective structure of the Internet is concerned, a PDF is essentially a dead end. Links only go into it, not out of it. Web crawlers, the text-reading bots with which search engines like Google collect texts, cannot read it. A PDF has no comment section, no Facebook "share" link. Lunsford's piece could perhaps have been shared anyway, but it had little reach: the Facebook automated programmer interface reports that, as of Fall 2015, the essay has been shared just seven times.

Thus, Lunsford's public-oriented argument never reached a significant public. Literacy researchers (including but not limited to Lunsford) appear to be silent when pundits blame American youth's supposed illiteracy on the digital revolution. Natalie Wexler (2015), for example, in The Washington Post, blamed Americans' purported inability to write on a lack of grammar instruction in our touchy, feely, overly techy schools - and proposed instead an atomized, acontextual form of writing pedagogy, that we return our pedagogical focus to teaching students to write sentences, which they must master before they can compose anything else (shared over 3,000 times in the first week of its publication). Nicholas Carr (2008) similarly worried in The Atlantic that hyperreading might be "making us stupid" (shared over 13,000 times since its publication). We thus remain outside of the public conversations about writing and writing instruction; even when our research speaks directly to concerns about writing, pundits do not seem to find that research. But as long as the knowledge we produce remains within our limited sphere of influence, we will continue to have the same tired conversations with the public: No, writing is not "getting worse," on average, and the rise of digital technology has not (or at least should not have) prompted a "literacy crisis. Not all writing about rhetoric online is off-target_Financial Times journalist Sam Leith 
keeps an engaging column about rhetoric called "The Art of Persuasion." Yet it seems strange to me that none among us have anything near Leith's reach. In Howard Tinburg's (2014) CCCC address, he called on the field to rededicate itself to the public. "As a learned society," he argued, "we stand committed to the production of knowledge that will, to borrow a phrase from our mission statement, 'enhance the conditions for learning and teaching college composition"” (p. 337). By not engaging in web opinion journalism in shareable, web-savvy ways, ${ }^{17}$ rhetoric and composition scholars squander an opportunity to educate the public about the work we do. In this dissertation as a whole, I argue that we need to go beyond a commitment to knowledge production, toward a commitment to knowledge dissemination. We must find ways to meet the public in the potent Internet forum for modern public debate.

I suggest, that is, that perhaps our apparent public silence on matters of writing research could be remedied if we worked to publish our findings in more web-savvy texts, ones designed to circulate. To understand what "web-savvy" practices are and how they affect circulation, we must consider the practices that characterize the kinds of text that circulate widely online: Short-form, networked web journalism. It is not enough, I argue, to publish online; even our journals that publish "webtexts" for the most part have not added Web 2.0-style reader engagement features, and they increasingly eschew hyperlinking. Hyperlinking is important to web writing not just because it is a standard genre feature; it is also a material manifestation of the intertextuality theories James E. Porter introduced to rhetoric in 1986:

\footnotetext{
${ }^{17}$ With, of course, some exceptions that prove the rule, including Hybrid Pedagogy. Note, however, that while Hybrid Pedagogy has web-savvy publication practices, it exclusively addresses an audience of educators.
} 
The most mundane manifestation of intertextuality is explicit citation, but intertextuality animates all discourse and goes beyond mere citation. For the intertextual critics, Intertext is Text - a great seamless textual fabric. And as they like to intone solemnly, no text escapes intertext. (p. 34)

Where intertextual theorists conceived of an Intertext connected by reference and allusion, the Internet enacts that vision more literally. Every node connects, if distantly, to every other node. The web is defined by each document's links to other documents, links that make it visible to search engines and thus available to for public consumption. My focus on hyperlinks in this chapter and in the dissertation more broadly came out of a suspicion that hyperlinking was not just a standard feature of web writing but also a key aspect of how that web writing circulates online. This chapter investigates that concern. In this chapter, I argue that understanding Internet web journalism's ever more standardized hyperlinking practices is prerequisite to entering that forum.

Those hyperlinking practices, though still in flux and often tacit, nevertheless exhibit some consistency. In the rest of this chapter, I report on my findings from an initial foray into hyperlink practices and circulation of web journalism. I have collected a corpus of approximately 4,000 articles each from Slate and Newsweek and analyzed the hyperlinks within; I have also interviewed two authors who write for Slate. My analysis demonstrates that there are standard ways of using anchor text, the words that point to a link (often underlined, bolded, and/or colored); most links have anchor text that is three words long or less. When authors break these conventions, they generally appear to do so for rhetorical effect, often to emphasize the main point of an article. Web authors write for a reader who may not read fully and may only click on one link in the whole article; thus, it is not uncommon to link the same source several times in a row. Perhaps most 
interestingly, there is a weak positive correlation between social sharing and link countwhich seems supported by authors, who tell me they add links to help their work's Google search rankings.

This project is an attempt at what Lev Manovich (2001) called "a new rhetoric of hypermedia." But, as Manovich predicted, the rhetorical affordances of the interface of hyperlinked text are most visible at the level of the interface: to understand how hyperlinks are used to argue, we must not just look at individual hyperlinks in individual texts. To get at this meta-structural level, I adopt a version of what Franco Moretti (2005) has called "distant reading": I look at a huge number of texts, to see aggregate patterns, to seek evidence of practices that have yet remained tacit. Insight into practices in aggregate is especially important in understanding a genre as new and developing as short-form networked web journalism; publications often do not yet have explicit guidelines for how and when to cite, and authors may not have been asked to verbalize their tacit understanding of these practices. Corpus analysis provides the broad, quantitative picture that I fill in with rhetorical analysis and interviews. Ultimately, this chapter suggests that such analysis will give rhetoric and composition scholars the tools to produce better, more web-savvy text online, which may be necessary for getting us involved in many of the national and international conversations where our work makes a difference.

\section{The Decline of Literary Hypertext Studies—and the Rise of Interface Studies}

As I detailed in Chapter I, there was much work done on the literary and rhetorical effects of hyperlinks in ergodic literary hypertexts in the 1990s and early 2000s (e.g. Bolter and Joyce, 1987; Joyce, 1996; Bolter and Grusin, 1999; Hayles, 2002). This work, however, focused almost exclusively on multi-part, modularized literary texts that 
could be read in a wide variety of orders, often (though not exclusively) created with the Storyspace software. Focusing on Storyspace meant that much of these early analyses discussed the changed relationship between reader and writer: readers had increased agency over the text, writers had to cede control over some aspects of the arrangement, and text could be circular or spiral-shaped or a haphazard hopscotch. Little or no concern was paid in these analyses to the anchor text itself, and because Storyspace began nearly five years before the development of the World Wide Web, "outside links" did not even exist until very late in the heyday of literary hypertext and hypertext criticism.

Yet online rhetorics have focused increasingly on the interface - the "frame" of an online text, which calls upon us to interact with it and structures the content within. Interfaces were first examined as ideological tools over two decades ago; Cynthia and Richard Selfe's (1994) "Politics of the Interface," for instance, considered how technological metaphors privilege particular orientations toward technology (e.g. the "desktop" proposes a white-collar attitude that a metaphor such as the "workbench" would not). As Web 2.0 has filled the Internet with more dynamically generated and usergenerated content, the focus on the interface as a rhetorical construct has intensified. Collin Brooke (2009) argued that the interface is where rhetoric and technology meet. He called interfaces the "excluded middle" of the typical English studies focus on either individual texts or larger theoretical structures: Interfaces, he said, provide examples of "rhetorical practices that may span multiple texts without achieving the level of abstraction of literary theory" (p. xvii). Teena Carnegie (2009) has taken up this call and discussed the way interfaces serve as a rhetorical "exordium," exhorting us to interact with a site. 
Brooke (2009) briefly discussed the interface of hypertext, specifically,

comparing link citation practices to academic practices:

We learn to situate our own work within those [academic] networks, providing links in the forms of citations, foot- or endnotes, and bibliographic entries. The texts that we produce bear a strong resemblance to individual readings of a hypertext: We choose particular paths through webs of scholarship, keep track of those paths, and account for them by publishing essays and books that then occupy a place within the webs that we have engaged. (p. 78-9)

The connection between academic parenthetical citation and link citation is key to my work: I see strong parallels between the ways that we read online and the ways we read academic work, and those parallels express themselves most clearly in citation practices. However, Brooke did not in this book-nor, as far as I have found, has any rhetoric and composition scholar yet — explore in detail the notion of a rhetoric of hypertext argument; such a rhetoric is the goal both of this chapter in particular and of the style guide that concludes this dissertation.

\section{Slate, Newsweek, and the New New Journalism}

Hyperlink citation came out of a strange antecedent genre spiral: prior to the rise of journalistic hyperlink citations, journalists did not cite (though they did, of course, attribute - naming sources, if vaguely, in text). The only extant model of formalized citation was academic, and thus, journalistic hyperlink citation practices necessarily drew some on academic models. In fact, the entire system of web search engines is based on the way academics cite. Sergey Brin and Larry Page (1998) explained that the algorithm behind Google, PageRank, is explicitly based on academic bibliometric models:

Academic citation literature has been applied to the web, largely by counting citations or backlinks to a given page. [...] PageRank extends this idea by not counting links from all pages equally, and by normalizing by the number of links on a page. (p. 4) 
Google's PageRank algorithm largely determines the distributive power of a piece of writing; whether or not something shows up in search results is dependent on how it links and how it is linked to. When web journalists are trained in any citation practices at all, they are taught procedures to optimize their writings' search engine results: link to sites with high rankings, put your links over keywords, and so on (Mathis-Lilley, 2015). Those practices came to some extent from academic bibliometrics. Thus, whether or not web journalists are aware of the connection between their work and academic practices, they have a shared history. And in return, as academic work moves online, we have had to draw on those developing hyperlink practices.

One very early case for hyperlinked journalistic practices was the online news and culture magazine, Slate, which began publishing solely online in 1996, just three years after the first graphical web browser was introduced. I originally chose Slate for my analysis of short-form networked web journalism because of its visibility and because of the sense that it has a unified style of writing. In my 4477-article corpus, there are 703 distinct authors; Slate nevertheless seems to maintain a pretty consistent editorial voice, one that I associate with Internet-socialized journalism, and one that seems to be increasingly adopted among post-web 2.0 media sources. That voice evidences a blogstyle casualness in tone, nevertheless backed up by thorough research. In 2009, Slate was the first web-only publication to win a Missouri Honor Medal for Distinguished Service in Journalism. Slate authors are writing opinion pieces, no doubt, but they are generally fairly well-sourced opinion pieces with the level of detailed reporting that readers expect from much more established print publications. 
Robert Boynton (2007) connected Slate to what he called "the New New Journalism," in an eponymous book. Where Thomas Wolfe's generation of New Journalists troubled the boundary between fact and fiction and brought literary attitudes to journalism, Boynton argued, the New New Journalists operate in a poststructural system that has already rejected objectivity—which gives them the freedom to opine without apology, to explore social constructions of race and class, and to approach nonfiction with a rhetorical and transformative social purpose. Boynton interviewed Michael Lewis, whom Slate dispatched in 1997 to write a 14-article series about the growth of Silicon Valley, called "Millionerds." The series is a prime example of the "drilling down into the bedrock of ordinary experience" that Boynton said characterizes the New New Journalism (p. xv); it is embedded journalism exploring not a war-torn country but a prominent American subculture.

Link citations in the early years of Slate were much rarer than today. Many articles in the early Slate had no link citations at all. Nevertheless, links were occasionally used in a way that resembles contemporary use: Lewis's (1998) profile of Xerox nanotechnology researcher Ralph Merkle, for instance, links to Merkle's "elaborate Web site" (the anchor text of that link, for what it is worth, almost precisely matches the average anchor text length of articles in my corpus), but does not link any other sources. In my corpus, on the other hand, Slate articles link on average 8.9 times per article. The most-shared and most-commented-upon articles often have more link citations, sometimes as many as 50. Somehow, between 1998 and 2015, link citation practices on the site have developed and begun to calcify. Yet, according to the author and editor I interviewed, Slate does not yet have formalized link citation practices; it does 
not have a documented style guide; and its authors and editors work on tacit understandings, developed through practice.

The second site I have considered in this analysis, Newsweek, I picked for both its similarities to and its differences from Slate. Both present short-form web journalism, publishing multiple articles daily, on a broad series of cultural categories from politics through sports through TV shows. Both address a broad, primarily American though also international audience. Newsweek's development of online publication practices, however, has been driven by a series of starts and stops, as it has moved in and out of print multiple times. It was first published in 1933, was purchased by The Washington Post in 1961, and was fairly successful through the early 2000s. By 2010, however, its readership had tanked and the company was deeply in the red — at which point, it was purchased for $\$ 1$ by the online news outlet The Daily Beast. In 2012, the publication announced its "last print issue" and moved to solely online publication. Still struggling financially, it was bought again in 2013 by the International Business Times-which, in 2014, returned the magazine to print. As of 2015, Newsweek's online content is behind a paywall, and both its print circulation and web traffic numbers have returned to competitive levels. The "audience-measuring" site Quantcast, which has official traffic data for Newsweek and a proprietarily calculated estimate for Slate, suggests that Newsweek web traffic has actually overtaken Slate's since the return to print (see Figure 2.1).

In addition to demonstrating several different trajectories of online publication, Slate and Newsweek also present different models for online monetization: Slate funds itself through advertisements, and all of its content is free. Newsweek funds itself through 
subscriptions - there are no ads on the site, and users can see 10 articles per month for free or pay $\$ 9.99$ per month for full digital access. I am interested in the ways that financial motivations influence publication practices: what sites publish, how they publish it, and how they cite sources within those publications. Because inbound and outbound hyperlinks are the primary metric in Google's PageRank site-sorting algorithm, the way sites cite can change the way they get ranked on Google — which in turn, of course, changes the amount of money they can make.

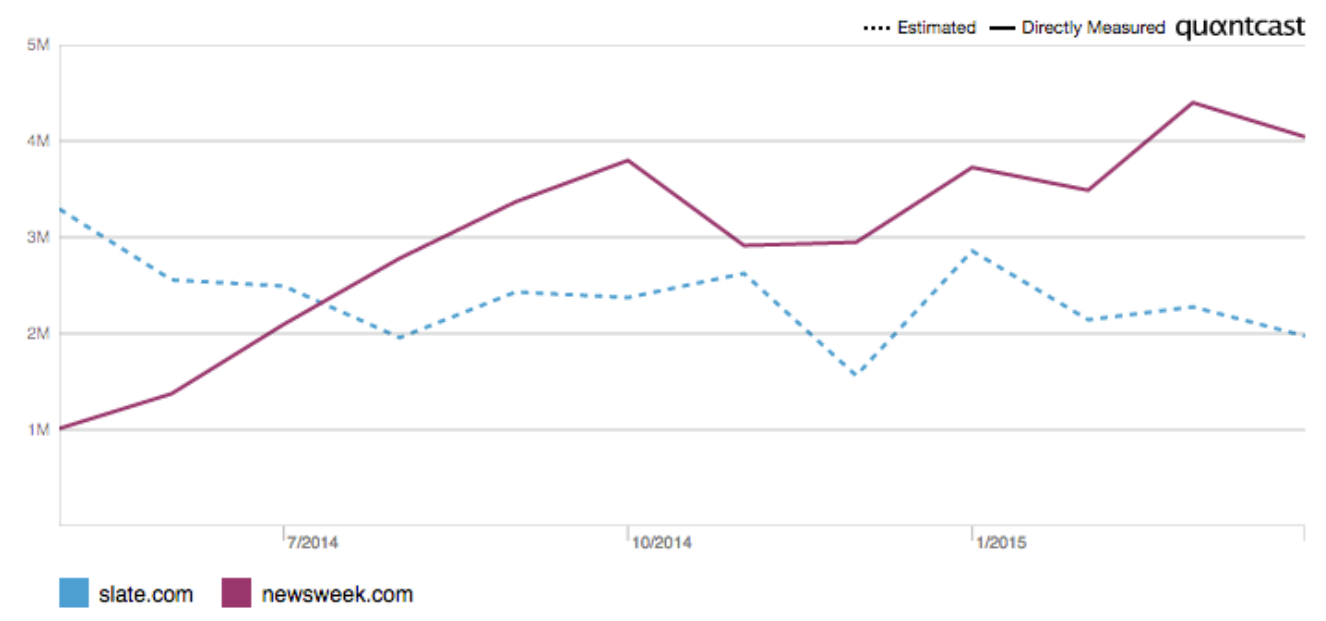

Figure 2.1: Quantcast's estimated traffic for Newsweek and Slate from May 2, 2014 through April 29, 2015, in millions of unique users. I include this dynamically generated graph to note two things: The rising trend of Newsweek traffic and the comparable range of the two sites' viewership.

\section{By the Numbers: Genre Features and Citation Practices of Web Journalism}

Several studies in the last 15 years have considered emergent web genres, cataloguing their formal features, considering their rhetorical construction, and exploring how they might be used in composition classrooms (e.g. Trupe, 2002; Miller and 
Shepherd, 2004; Edwards and McKee, 2005; Palmquist, 2005; Kynard, 2007). Little attention has been paid, however, to web journalism specifically, despite its ubiquity and influence. A better understanding the genre of web journalism will offer our field at least three tangible benefits: (1) we can better teach the genre, which is increasingly important as more schools offer professional writing and web writing courses; (2) we can more effectively enter the space of and/or imitate the features of web journalism, in ways that might help us reach wider publics; and (3) we can understand some of the features and qualities that make web genres circulate as they do, perhaps again finding ways to appropriate some of that rhetorical velocity in our own work.

I argue that Slate and Newsweek articles share a genre (or, perhaps, genres) in part because they share many of what Frow (2006) terms "formal features." For instance, the articles in both the Newsweek and Slate corpus have similar mean word counts: the mean Slate article has 639 words, and the mean Newsweek article is just a little longer at 690 words. This average aligns both with general trends in journalism and with recommendations for web journalism particularly. A Columbia Journalism Review study found that longform journalism is in decline, with stories over 2000 words as a percentage of total stories falling dramatically in three out of four major newspapers between 2003 and 2013 (Starkman, 2013); a Medium study of optimal web content length determined that user engagement peaks at around seven minutes per article, or about 1,600 words (Sall, 2013). Each article on both sites is paired with at least one "header" image that sits at the top of the article's page and serves to represent the article metonymically, both throughout the site (in section summaries, in "related article" features) and on social media; many articles on both sites have more than one image, that 
shows up alongside the content later in the article. The sites also share a significant number of their section names (and thus both topics and topoi): each has sections labeled "Technology," "Science," "Politics," and "Education"—and even where sections do not directly align, they often share a similar spirit (what Newsweek calls "Religion" is "Faithbased" on Slate; Newsweek's "Sports" is Slate's "Sports Nut"). ${ }^{18}$

Furthermore, the sites share what Frow (2006) terms a similar "situation of address": On both sites, texts respond to a demand for timely, topical news and opinion content. Most authors on both sites are full-time, regular contributors to a particular section of the site, given leeway in picking their article topics, but expected to keep up with developments related to their section and regularly publish according to deadlines. Other authors on both sites are one-time or infrequent contributors, having proposed (or been invited to write) an article on a particular topic. They reach readers seeking brief newsy diversions, on their phones and computers, accessed via either app or web browser. Thus, they share a physical setting, also; Slate and Newsweek content may even end up quite literally interleaved, when the sites' articles reach other readers via RSS feeds, where articles are displayed without the site's formatting and other trappings, presented to readers alongside other content from similar kinds of sites. Yet other readers encounter articles from each site first on social media including Twitter and Facebook, where the article is represented via a friend's comment or summary; on Facebook, this comment is paired with the article's headline, a representative image, and a brief subheadline. Quantcast suggests that both sites have average monthly viewership that ranges from about 1 million to about 4 million (see Figure 1). Another Internet traffic-

\footnotetext{
${ }^{18}$ Slate, however, has 95 categories to Newsweek's 16 . The more explicitly cultural focus of Slate is evident in these titles, including separate sections for movies, books, television, music, and gaming; on Newsweek, articles on these topics are all rolled into "Culture."
} 
measuring site, Alexa, suggests that both Slate and Newsweek draw more than half their viewers from the United States ${ }^{19}$.

I mean to make several points, in drawing this comparison: First, of course, that the sites are close enough in goal and purpose to make sense to consider together; because they share a genre (or genres), they can be compared meaningfully, as I will proceed to do in the rest of this chapter. More importantly, though, I mean also to demonstrate that articles on both Slate and Newsweek are examples of the kind of "posthypertextual" short-form web hypertext that I introduced in Chapter I and that I will propose, in Chapter V, that rhetoric and composition should aim to create.

\section{Citation Practices that Characterize Web Journalism}

My analysis of link citation practices on Slate and Newsweek suggests that, although the authors I interviewed said there were no explicit guidelines for how to cite, the sites have nevertheless developed some standardized link citation practices. The vast majority of articles on both sites have between one and ten links (see Figure 1). Link citations on both sites have anchor text that is, on average, about three words and about twenty characters long (so, this is typical but it is much less common to see a whole sentence like this linked). On Newsweek, $71.3 \%$ of links have anchor text that is three or fewer words; on Slate, $61.6 \%$ (see Figure 2.2). Compared to traditional academic parenthetical citation, the brevity of the hyperlink citation is interesting: it is a condensed intertextuality. Even more explicitly than most parenthetical citations, the hyperlink citation functions metonymically - a small couple of words usually stand in for the

\footnotetext{
${ }^{19}$ Unfortunately, no more granularity regarding the demographics of the sites' users is available to the public.
} 
entirety of a linked source. Or, where a quote from that source is included, the link gets tied nevertheless to the reporting verb rather than the entirety of the utterance. 
Slate and Newsweek: Number of Words in Anchor Text

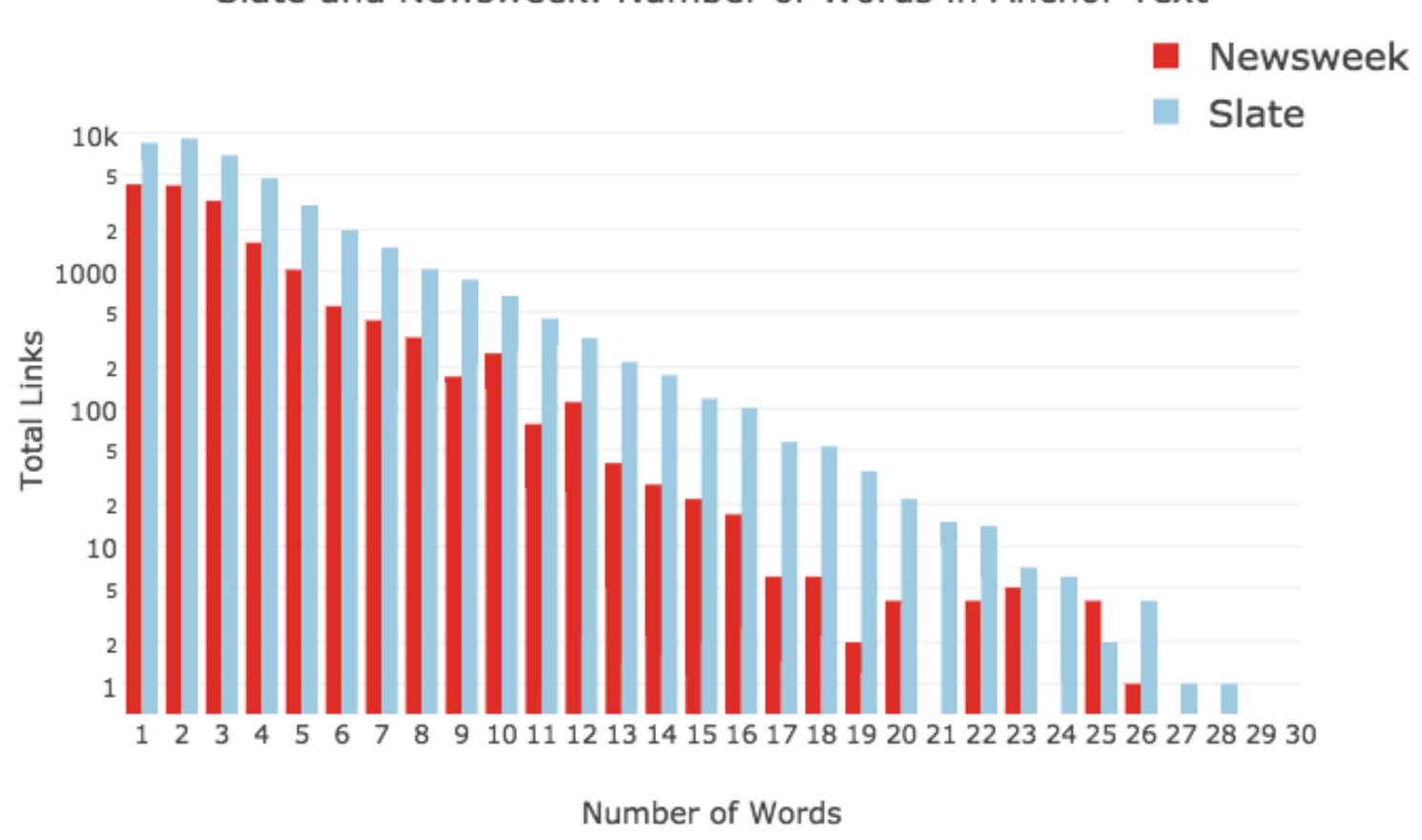

\section{Link Anchor Text with 3 or Fewer Words}
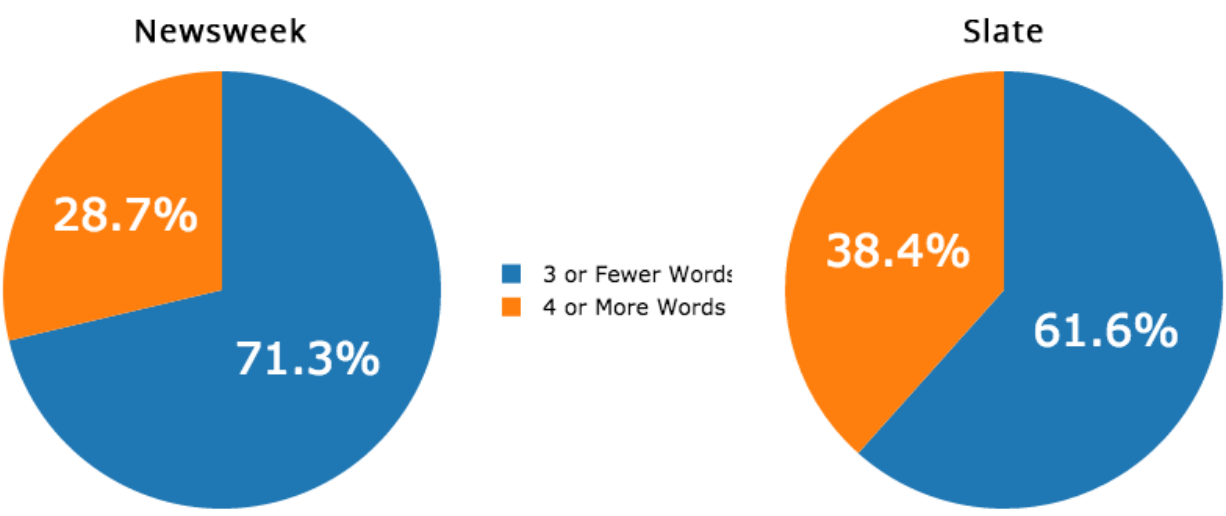

Figure 2.2: The majority of link citations on both Newsweek and Slate have anchor text that is three or fewer words long. After a slight peak at two words long, each additional word count is less common than the previous. 


\section{Links Are Associated with Argument}

Given that citations often serve to support claims, and claims are used to construct arguments, my finding that higher link citation counts are associated with arguments is perhaps not surprising. Slate articles tend to cite slightly more frequently, a median of seven links per article, compared to Newsweek's three. Significantly, Slate publishes more opinion journalism and less newsy reporting than Newsweek. Newsweek's numbers are lower in part because, in addition to its many original articles, the site republishes Reuters wire pieces without additional comment (Reuters pieces have no links and are designed for print redistribution as well as web redistribution). Even discounting Reuters pieces, however, Newsweek articles still cite less frequently than Slate's - with one section as exception: Newsweek's "Opinion" section (see Figure 2.3). Whereas every other section on Newsweek has a mean link count per article under six, the mean link count in the "Opinion" section is 13.5. 
Newsweek: Average Link Count by Section

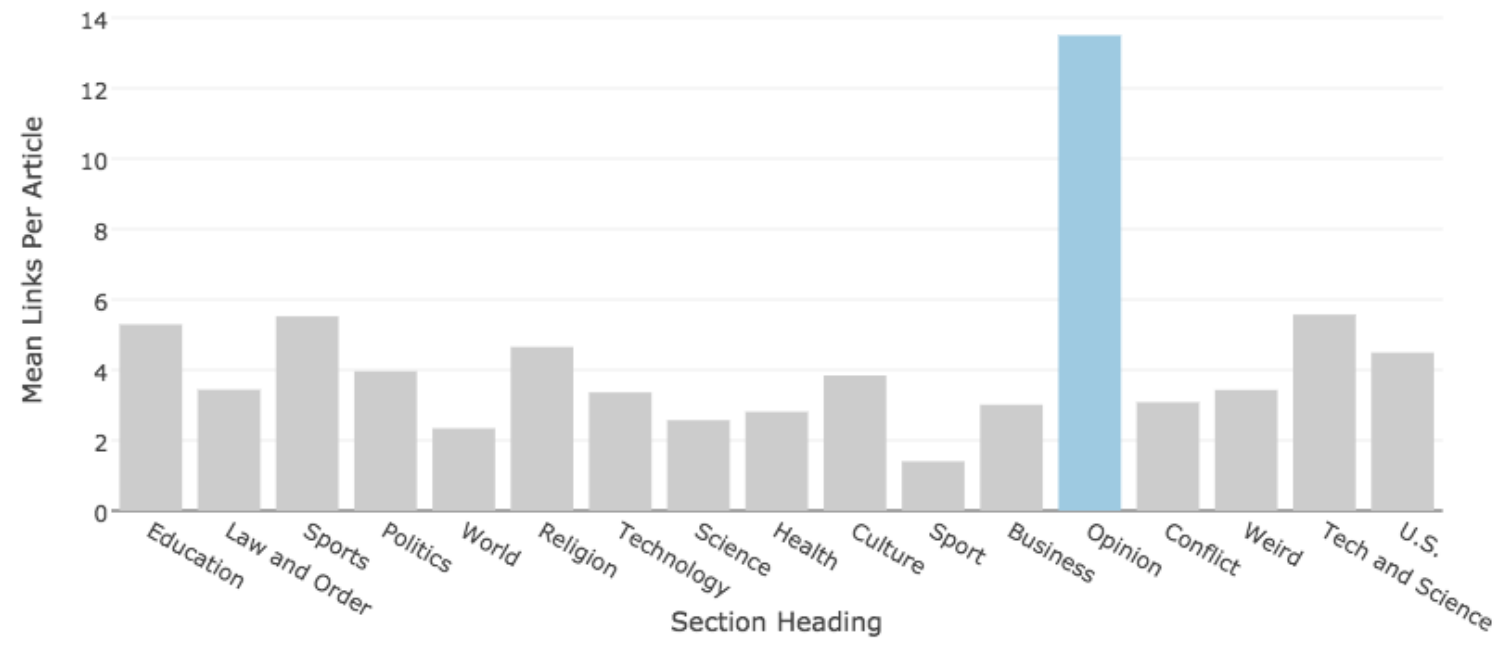

Figure 2.3: Average link count by section on Newsweek. The "Opinion" section's mean link count is more than double that of the next-highest section.

Links, thus, appear to be a key feature of online argument; the genre has changed significantly since Manovich (2002) claimed that links are arhetorical. Another potential contributing factor to Slate's higher link counts than Newsweek's, however, is the fact that Slate is funded by advertising rather than subscriptions. Because a higher quantity of quality links tends to boost Google search rankings, some Slate authors may link more in part because they know those practices help support the site; Ben Mathis-Lilley, editor of The Slatest and the most prolific author in my corpus, noted that an editor at his previous position (BuzzFeed) had told him to link "keywords" to boost his articles' searchability. 
While Newsweek is, of course, also affected by Google search rankings, its funding model is not as directly dependent upon search engine optimization.

The notion that Slate authors link more often to increase the shareability and visibility of their articles is potentially supported by another finding in my data: Higher link counts are associated with higher social sharing, to a point. Figure 2.4 shows link counts per article graphed against total shares, likes, and comments; I have grouped the articles in buckets of 20 for readability, but the trend is still evident even in much smaller groupings. 
Newsweek: Facebook Share Data by Link Count

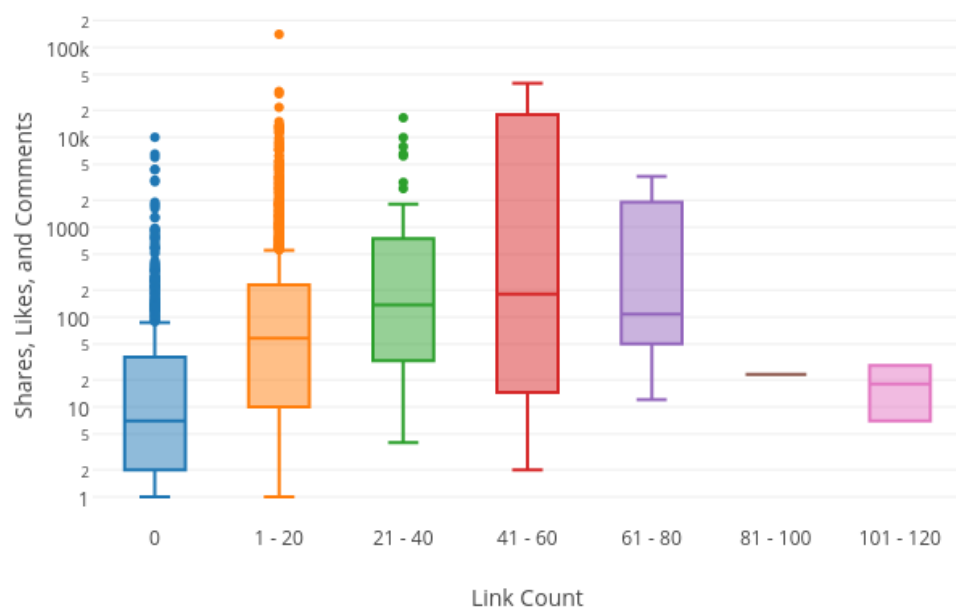

Slate: Facebook Share Data by Link Count

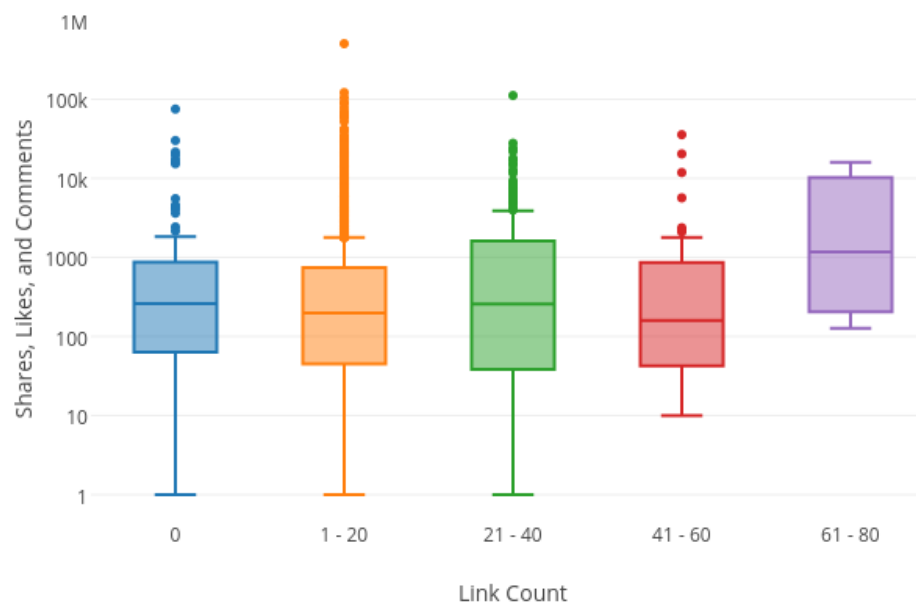

Figure 2.4: Higher link counts per article are associated with higher share, like, and comment counts. The $\mathrm{X}$ axes on both graphs are logarithmic because the range of each is so large. Each of these box-and-whisker plots buckets link counts in groups of twenty, for clearer visibility of the trend. NOTE: The program used to generate the Slate graph above is in Appendix D. 
As evident in Figure 2.4 above, on Newsweek, the median share count rises pretty steadily through 41-60 links per article, then drops somewhat after that. There is not enough data after 60 links per article for that drop to be significant—only one article is in the 81-100 link grouping, and only two are within the 101-120 link grouping. ${ }^{20}$ On Slate, the median share values rise less steadily. Articles with 21-40 links have a median count of 257 shares, likes, and comments, which is higher than both articles with 1-20 links (median of 197) and articles with 41-60 links (median of 159). The highest median share count by far, however, is in the 61-80 link grouping, at 1168 shares, likes, and comments.

Several circumstances might account for this correlation between high shares and high link counts: as suggested above, links might improve the Google ranking of particular news articles, which might increase their visibility. Or, perhaps articles with higher link counts tend to be more thoroughly researched, more carefully constructed and written. Given that higher link counts are associated with argument, it is possible that authors link more when making controversial arguments. Controversial arguments may circulate further and generate more comments, because they provoke people to respond or align themselves with or against.

\section{Most Articles Do Not Link to Other Articles on the Same Site}

Though some link citation practices do suggest that authors on these sites are constructing their arguments to improve the sites' Google rankings, apparently this practice does not usually include enforced linking back to articles on the site of publication. I predicted that, because eyeballs are dollars (especially on Slate, which

\footnotetext{
${ }^{20}$ Those two articles in that last bucket are, interestingly, part of a short-lived series of articles about 2016 presidential candidates: "Marco Rubio: All You Need to Know" and "Hillary Clinton: All You Need to Know.”
} 
survives solely on advertising and Amazon affiliate revenue), the majority of articles would include links intended to keep readers from leaving the site. In my brief experience writing for web magazine publication (in places including The Atlantic and Wired), editors would often add in a link to other content from the site of publication; the Treehugger content management system requires authors to choose items for the "related articles" section that appears underneath each article.

On both sites, however, under $20 \%$ of articles link back to other articles on the same site ( $13 \%$ on Newsweek, $18 \%$ on Slate), far from the $80 \%+$ I predicted, based on my experience. While the designs of both Slate and Newsweek present viewers with plenty of links to other site content (including featured and trending articles, suggestions for similar or related articles, and a range of section headings), the articles themselves are much more often than not independent of other articles on the site. Thus, the data do not support my hypothesis that editorial demands lead authors to link to the site even where not relevant.

\section{Rhetoric of Hypermedia}

The relationship between the linking text and linked text can vary significantly from link to link—-some links are presented definitionally or encyclopedically (as on Wikipedia), others serve to expand upon or defend a point somehow. Nicholas C. Burbules (1998) attempted to categorize the various "tropes" of "semic relation" in hyperlinks (p. 104); "The link," he said, "is the elemental structure that represents a hypertext as a semic web of meaningful relations" (p. 105). It constructs that web rhetorically, he argued, using familiar tropes of rhetorical semic relations. Burbules identified and demonstrated eight semic hyperlink tropes: metaphor, metonymy, 
synecdoche, hyperbole, antistasis, identity, sequence/cause \& effect, and catachresis. Metonymic relations, for example, are frequently evident in interface design, as we use older media and non-technological symbols to help us understand technology; for instance, we speak of a website's index as "home" and even represent it with a houseshaped pentagon. Catachresis, for another example, is the linking of two otherwise distinct notions or ideas, what Burbules calls "far-fetched associative links" (p. 117). Internet culture has, a decade after Burbules wrote, delivered up the consummate example of a link with a catachrestic relationship to its subject: the meme practice of "Rickrolling," in which a writer links Rick Astley's 1987 music video for the song "Never Gonna Give You Up" in a context where it would otherwise be irrelevant. "The act of a link," Burbules argued, "is not simply to associate two givens" (p. 105). Rather, the link performs a particular relationship between those two things. In a later essay, Burbules (2002) argued that the inseparability of links' semantic and navigational functions is key to what makes the web a "rhetorical place" (p. 75), in that links serve both "as avenues of movement and as occasions for meaning-making" (p. 82).

My following investigation of the rhetoric of hyperlinking is intended to build upon Bubules's categorizations of hyperlinks' "semic relations"; unlike Burbules, however, I do not focus primarily on the relationship between the citing and cited textswhile an interesting axis of comparison, and one I might aim to take up another time, I focus here instead on the role of hyperlinks within an argument. I consider the ways that the ordering, naming, and presentation of links functions rhetorically; this is an aspect of

\footnotetext{
${ }^{21}$ This meme entered mainstream consciousness when Rick Astley performed "Never Gonna Give You Up" on a float in the 2008 Macy's Thanksgiving Day Parade.
} 
rhetorical linking that has not yet been the focus of study. I build on this rhetoric in the "rhetoric of hypermedia" style guide that concludes Chapter V of this dissertation.

\section{Don't Click Here: Semantic Anchor Text}

Perhaps one reason hyperlinks have not killed rhetoric in the way Manovich (2001) predicted is that links no longer serve to disrupt the arrangement of the original text. Not only are internal links (which "modularize" text) less common outside of Wikipedia; even external links do not send readers down the rabbit holes they might have fallen into fifteen years ago. When I talk to people about their online reading habits, most claim to click links in news articles only rarely. The force of a link thus often stands separate from its linked content. The bare fact that something is linked is often adequate to convince readers that a statement is sourced; the somewhat curious reader might hiver over a link to see where it leads; only the most powerfully compelled readers actua.

Furthermore, link anchor text content is generally semantic: no news source would entice you to click here for more information - without any more information about what "here" might be. Rather, from the anchor text, readers often can guess what type of source a link might be. Bella Dicks, Bruce Mason, Amanda Coffey, and Paul Atkinson (2005) argued that "consistent, principled use of link names [as they term anchor text] can help construct a semantic map of the hypertext," making readers cognizant of the relationship between the source and target pages (p. 46). Consider, for instance, a phrase from one of the most-shared Newsweek articles in my corpus, Zoë Schlanger's (2015a) "NASA: California Has One Year of Water Left": near the opening of the article, she wrote, "In an op-ed published Thursday by the Los Angeles Times...," which leaves no question what a reader who clicks the link will find. 
This example particularly demonstrates both the uninterruptivity and the semantic nature of links, because Schlanger's article is essentially a summary of the LA Times oped. The linked op-ed is the primary source of Schlanger's information; 129 of the article's 442 words are direct quotes from the op-ed, and all but one paragraph appears to draw information immediately from it. The op-ed is only marginally longer, at 670 words, and was written by a NASA/JPL scientist. Yet Schlanger's summary has been shared nearly half as many times as the original op-ed: Schlanger's article has been shared 4,954 times, compared to the original piece's 11,569. Indeed, the fact that the article has been shared and commented upon so many times (with 4,954 Facebook shares, 77,185 Facebook likes, and 57,696 comments at the time of data collection) is perhaps the strongest indication of all that people are not clicking links. If people were clicking through, that is, it seems much more likely that they would share the original op-ed rather than Schlanger's article. ${ }^{22}$ Presumably, many of those people encountered Schlanger first and did not click through to the op-ed. Most readers will likely accept Schlanger's summary of the op-ed without clicking away from the Newsweek article just a sentence and a half into it. A reader who wants to know more might open the LA Times op-ed in a tab behind the current one, for perusal after finishing Schlanger's article. The link, thus, does not necessarily interrupt the arrangement of Schlanger's argument.

\section{Link Chaining: An Affordance of Uninterruptivity}

Robert Connors (1999), in “The Rhetoric of Citation Systems Part II," described how important the interruptivity of citation systems was in their development: Until the

\footnotetext{
${ }^{22}$ Slate, of course, has a more national and international audience than the Los Angeles Times, which could also account for some of the disparity.
} 
mid-20th century, all citation styles had been uninterruptive, foot- or endnote-based. Parenthetical citation systems, by contrast, Connors argued, "relegate issues of readability and prose style to tertiary importance [...] Many authors in the sciences and social sciences felt at first that parenthetical citations disrupted and even degraded their prose style and the experience of their readers" (p. 240). Because link citations do not interrupt the flow of text at all—but offer the potential for full "elective access to the citation"- Connors suggested that hyperlink citations are "a sort of natural extension of marginal notes" (p. 220).

The clearest example of the uninterruptive affordance of hyperlink citations is in what I call "link chaining," a common citation practice in web journalism. Authors will often cite several sources in a row, with only a word or two between the linked phrases to visually separate them. In a widely shared Newsweek article, for instance, Miriam Boeri (2015) said, "Certain mental illnesses, such as antisocial personality and bipolar disorder, are found to predispose some people to use drugs." Cited parenthetically, the visual weight of this sentence would be either overwhelming, with parentheses after each linked phrase, or confusing, with three semicolon-separated citations at the end of the sentence. Instead, Boeri is able to reference three academic studies without skipping a beat: Interested readers are just a click away from full publication information, including an abstract, for each article. Those willing to take Boeri at her word (a word that is deemed credible thanks in part to her voluminous link chaining) and uninterested in reading sources will not have their reading interrupted with heavy parentheticals or the parallel discourse of an endnote system. Hyperlinks add a level of accountability to news journalism, which had previously dismissed citations in favor of prose readability. And 
unlike citation strings at the end of a sentence, link chains leave no question about which idea is supported by which referenced work. Of course, with any system, there are tradeoffs - because hypertext readers are not bothered by citations, they may not be bothered to vet those citations and may be more susceptible to false claims or other misleading practices.

\section{Link It Again, Sam: Doubling Links}

There is potential for great deception, for example, in the fact that all links look the same. Authors can cite the same source six times without raising red flags. This kind of multiple citation of the same work is apparently quite common among articles in the corpus. The Schlanger article above also exemplifies another interesting category of link citation practices: she links the same source twice in a row, with two different pieces of anchor text. In the one paragraph that is not drawn directly from the LA Times op-ed, she said,

Last month, new research by scientists at NASA, Cornell University and Columbia University pointed to a "remarkably drier future" for California and other Western states amid a rapidly-changing climate. "Megadroughts," the study's authors wrote, are likely to begin between 2050 and 2099, and could each last between 10 years and several decades.

\footnotetext{
"New research" and "megadroughts" both link to another article by Schlanger (2015b), from February, called, 'U.S. Soon to Face Worst 'Megadroughts' in a Millennium, Scientists Predict," which summarizes a Science article. The first piece of anchor text calls attention to the recency and academic credibility of the source; the second piece of anchor text makes clear that the linked source will define and explain the term "megadrought." A link can only point to one source and vice versa-it can only be
} 
attached to one anchor text phrase at a time. This is significantly different from academic parenthetical citation, in which it is common to cite several things at the end of a sentence or paragraph without neatly tying any one piece of it to any one particular source. To cite the same thing again, an author must link the work again, attaching it to a new piece of anchor text.

Rebecca Schuman demonstrates a similar linking practice in an article about “5+2” programs, which offer PhD graduates a two-year post-doctoral teaching position designed to fund them while they begin an academic job search. Schuman's article at one point cites, twice in a row, an MLA report:

Among the findings of a 2014 Modern Language Association report on the future of graduate study: It currently takes far too long to complete a humanities Ph.D.the median completion time is an astounding nine(!) human Earth years.

Like Schlanger's double citation, Schuman first links the report, then links again to the report in mentioning a particular key concept. When I pointed out this double link to Schuman in our interview, she suggested that the practice served both to meet the expectations of readers who may not be deeply engaged and to demonstrate that she has read the report she is citing. She described her choice to link again thus:

Basically, on the Internet, you have to assume your reader is not going to click on very many of your links. So, every time you cite something, you have to assume it's going to be the only thing your reader reads, if at all. When you talk about a big report, the convention in Internet journalism is that you link to that report immediately [...] The first citation is to allow other people to read it if they wish; the second is to show people that I read it and I know what I'm talking about. [...] The second one is substantiating.

Two aspects of this response seem important: First, supporting the notion that link citations are intended to be non-interruptive, she suggests that Internet writers assume 
readers do not generally click links. Second, she suggests that the practice of pulling a particular item from a report—and reminding readers via the second link that it comes from the report linked above — establishes her own credibility and the credibility of the phrase linked.

\section{Pay Attention to This Phrase: Materiality of the Underline}

I argue that in both these "double link" cases, the second link serves yet another rhetorical purpose: While it would be clear from context that the second sentence in each case came from the same source, another link allows authors to emphasize a key point, an idea that is particularly important. Hyperlinks are indicated by some sort of stylistic difference in the text — an underline, a color change, bold font, or sometimes all threethat necessarily draws a reader's focus to the linked phrase. Links offer a means of visual emphasis, in a genre that generally eschews the casualness of italicizing or bolding.

By linking the word "megadrought," Schlanger does not just let curious readers know that a definition lurks behind the link; she also calls attention to that word. It is the key concept of the only paragraph that is not drawn directly from the source LA Times op-ed, and it links to another article by Schlanger (2015b) about megadroughts. The source op-ed uses the word "mega-drought" (hyphenated as such) once but does not define the term or discuss large-scale droughts in other western states. Thus, as Schuman suggests, the "megadrought" link helps establish Schlanger's credibility, prove that she has read more about the topic. Yet it also coalesces the article's focus around megadroughts.

Schuman's second link, “astounding nine(!) human earth years," similarly calls attention to a key concept of both the paragraph and the article: the length of time to 
completion of a humanities $\mathrm{PhD}$. Note, too, that this anchor text is well above average length (36 characters, nearly double the 20-character average), which calls further attention to it, as does the parenthesized exclamation point.

The emphasizing of the materiality of a hyperlink's underline could be exemplified by practically any article in my corpora — most articles, that is, have some phrase that appears to be linked primarily to draw attention to the phrase itself. I will draw a couple more examples, from a highly shared article each on Slate and Newsweek. ${ }^{23}$ The most-shared Slate article in my corpus (at 121,167 shares, likes, and comments), for instance, debunked a research study that found beards are "as dirty as a toilet" (Coil, 2015). In the opening paragraph, the author joked that the study "hits almost all the sweet spots of the [germophobia study] genre"-it has "no actual data, no controls, nonsensical interpretation of results." "The only missing ingredient," Coil explained, "is that this study was conducted by a TV news station instead of the usual: a company that produces cleaning products." This first linked phrase is even longer than Schuman's long anchor text string above; at 45 characters, it is more than double the 20 -character average. No other linked phrase in the article is more than 20 characters long. Consider the changed rhetorical effect had Coil linked only "this study," which might counterintuitively lend legitimacy to the study, by separating the study from its dubious source. Alternatively, Coil could have linked something in the following sentence, which explained the procedure of the study — but again, this would have drawn attention to the study itself rather than Coil's critique of it. Instead, the linked article does not verbatim support the claim in the anchor text phrase; the linked phrase is Coil's critique of the

\footnotetext{
${ }^{23}$ I have chosen highly shared articles to understand citation practices in articles that have achieved rhetorical success, but I do not have the data to suggest a causal relationship between those articles' citation practices and that virality.
} 
study's source rather than a description of the study's technique. Linking the entire clause emphasizes the main point of the article.

There is a similar "thesis" link at the end of the introduction of the most-shared Newsweek article (at 40,072 shares, likes, and comments), which is titled, "Marijuana Is Not, Repeat Not, a Gateway Drug” (Boeri, 2015). The second-to-last sentence of that introduction reads: "Research shows that the vast majority of marijuana users do not go on to use hard drugs." Again, Boeri could have linked "research shows," but instead chose to underline the 30-character argument. Boeri includes just a few similarly long links; most are just a word or two.

The materiality of a linked phrase, however, goes beyond the materiality of the underline itself. In a personal interview, Slate author and editor Ben Mathis-Lilley told me that he prefers to link words that he deems "keywords"-words important to the main point of the article - because an editor at Buzzfeed, where he worked before Slate, required it:

I used to link the keywords, words that were probably 'topic words' of a certain subject, because one of our audience engagement editors had said that helps your Google ranking. You get a better Google result when your links are in keywords. [...] I'd make sure the link went over those things that people might be searching for, or what the Google algorithm might be looking for, whether it's Google News results or what have you.

Thus, linking the entirety of a "thesis" as opposed to a subject-verb pair like "research shows" might actually change the way an article circulates by determining whether and how it gets listed in Google results.

\section{"Unsourced" and "Anti-Academic": Academics Respond to Rebecca Schuman's}

\section{Hyperlinking}


Rebecca Schuman understands quite personally both the challenge of Google visibility and how the citation practices of the humanities must be adapted for web journalism. Schuman was a self-professed "golden child" of German literature (personal interview, July 13, 2015): She won a Fulbright scholarship to study in Austria. She finished her dissertation on time. She published. Yet, after four years on the academic job market, she was not hired. She drifted from post-doctoral work to adjuncting, chasing the tenure-track promise. After that fourth failed year on the market, she wrote a long Facebook post, telling her story and discouraging her friends from attempting to enter academia. One of those friends was an editor for the online culture magazine Slate - and he asked her to turn the post into a 1500-word piece for the site. When she floated the idea among her academic colleagues, they strongly discouraged her. "When you go back on the job market, this is going to be terrible for you," they said. "You will have a Google problem."

She published the piece anyway, in April 2013: "Thesis Hatement: Getting a Literature Ph.D. Will Turn You into an Emotional Trainwreck, Not a Professor.” It went quickly viral. "I went from nobody knowing who I was to getting 10 angry emails a day," Schuman said. At first, the academic backlash frightened her; she worried that she had torpedoed her chances of gainful academic employment. Then, Slate offered her a contracted position, writing about academia for the magazine. Over the last two years, she has published a couple of pieces a month, examining and critiquing trends and events in academic culture.

Academics have, in turn, critiqued Schuman - the most virulent of these critiques is Charles Green's (2014) Inside Higher Ed "The Anti-Academic's Anti-Academic," 
which accuses Schuman of making "anti-academic" arguments. Her arguments, Green claimed, "rely heavily on unsourced and unsupported generality clothed in hyperbole." The suggestion that she does not research or does not research well is, she says, the critique of her work that hurts the most. "It didn't take me a year to write each of my articles," she explained, "like it did to write my academic articles, but I do research them very rigorously."

This critique is particularly interesting given that Schuman cites more often than the average web journalist: her articles in my corpus cite approximately 26 times each, much higher than Slate's median 7 links per article and Newsweek's median 3 links per article. As Figure 2.5 demonstrates, Schuman's link average puts her deep in the long tail of link count distributions: 


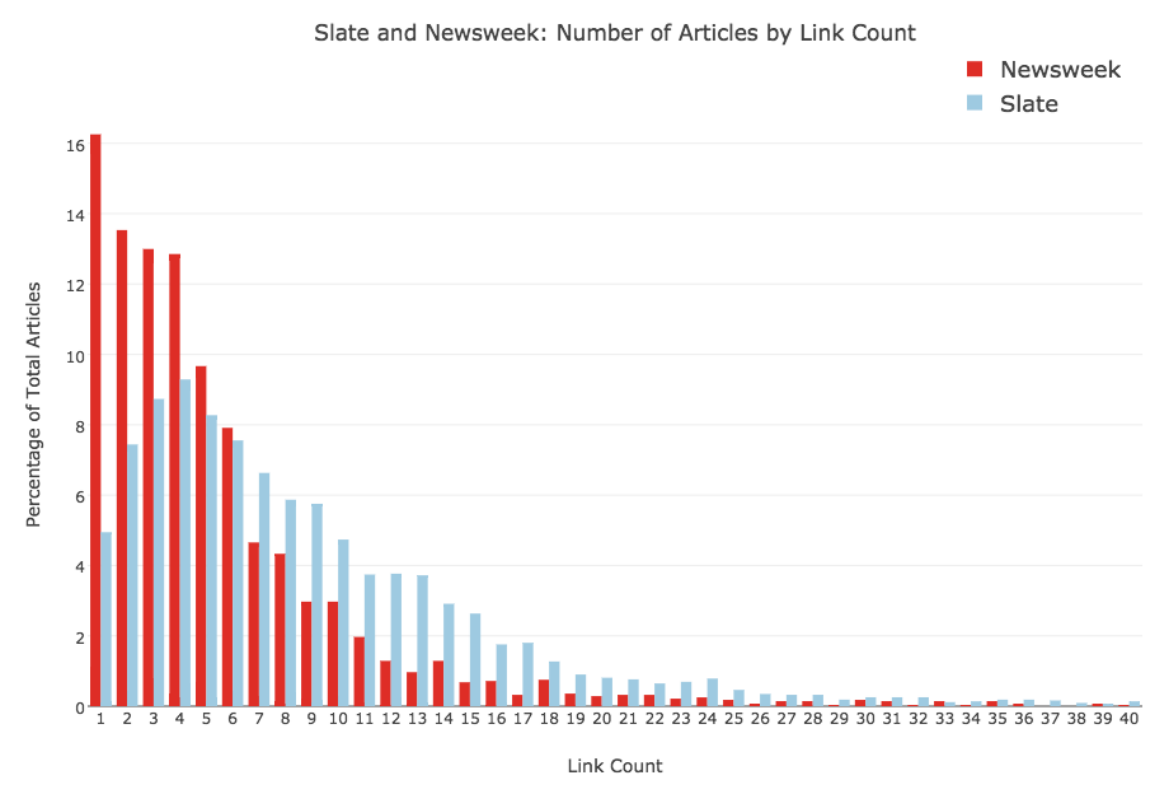

Figure 2.5: The percentage of total articles that have a given number of links, in Slate and Newsweek. Note Newsweek's precipitous drop from a peak at 1 link per article, and Slate's peak at 4 links per article.

Though I do not wish to make the specious claim that a high link count necessarily equates to good research, clearly Schuman's writing is not "unsourced" by the standards of web journalism. Pointing to the rise of web-savvy academic text like the peer-reviewed journal Hybrid Pedagogy, Schuman suggested that academic citation practices are likely to begin to change: "If and when academic prose becomes more online," she said, "more standard, and people start taking advantage of the hypertext milieu, their citation practices may change."

Schuman's experience thus exemplifies an important tension: how what counts as research and what counts as well-supported argument differs online and in academic 
print. She also has significant insight into both web and academic citation practices, and she represents one potential path for how academics might use web journalism to engage in international debate. In Chapter V, when I develop my proposal for a new kind of academic web publication, I return to several key aspects of Schuman's experience: The way that being a gadfly, being deliberately provocative instead of academically circumspect, can help an author online gain attention and traction; the way that academics evidence some suspicion of Schuman's web citation practices, perhaps in part because they share the values of web writing I will discuss in Chapter IV; and the way that Schuman uses her liminal position as ex-academic to reach readers on both sides of the academic aisle.

\section{Headline Grammar: What Else Might We Learn from BuzzFeed?}

Finally, I want to conclude this chapter with one last data-driven foray into the question of how best to reach that kind of wide audience: Ben Mathis-Lilley's comments about learning practices that contribute to high social sharing from his experiences at BuzzFeed led me to consider what other web practices might contribute to articles performing well on Facebook or Twitter. Knowing the prevalence of BuzzFeed headlines that directly address the reader (“Are you making your friends angry?") and that describe the editorial team with the words "we" and "us" ("Can we guess your age based on this test?"). Thus, in Figure 2.6, I charted average shares, likes, and comments for Slate and Newsweek articles with particular pronouns in the headline. 
Slate: Does pronoun use affect social sharing?

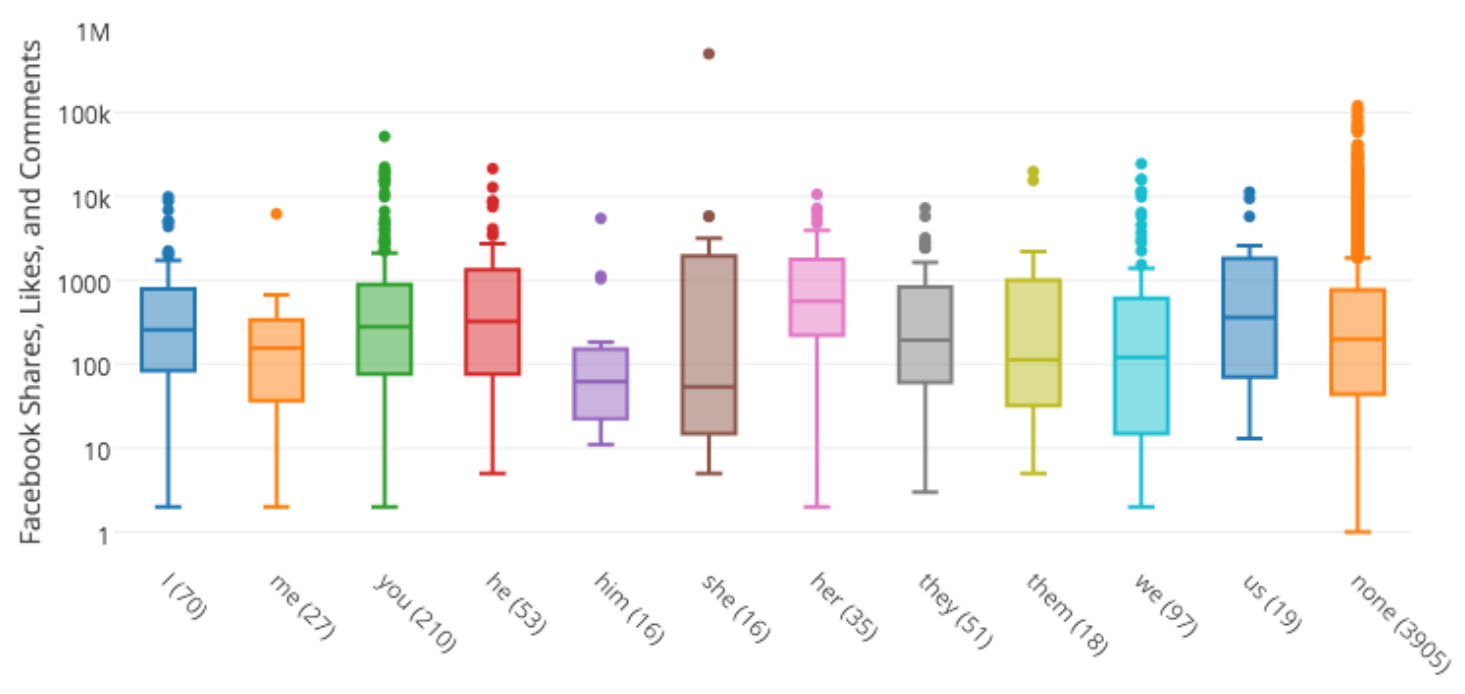

Newsweek: Does pronoun use affect social sharing?

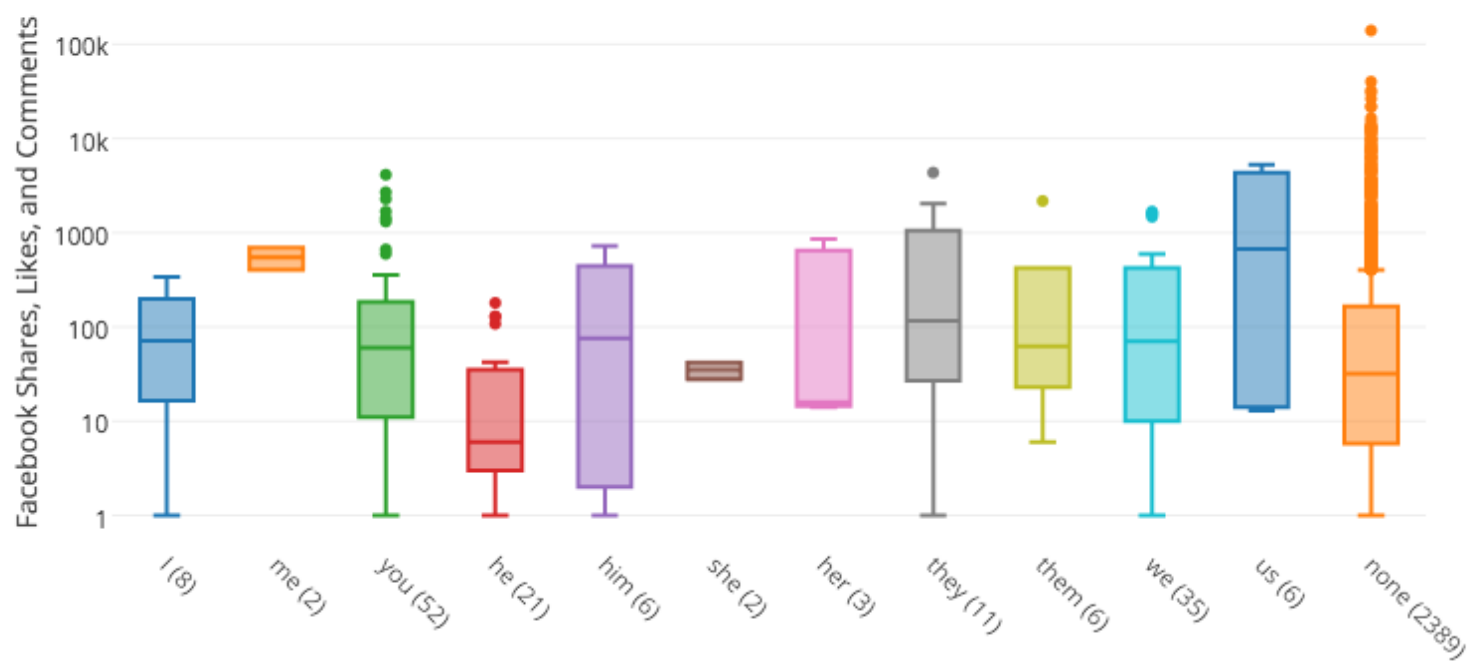

Figure 2.6: A series of candlestick charts showing Facebook shares, likes, and comments for articles with particular pronouns in their headlines. Note that on both Slate and Newsweek, headlines with "I," "you," and "us" perform better than the site-wide average (far right orange candlestick, labeled "none"). 
Though there was significant variation across the two sites, headlines with "I," "you," and "us" performed significantly better than average on both sites. Of course, I do want to be wary of generalizing from too-small a sample size; on both sites, the number of headlines with pronouns was far, far smaller than the number of headlines without.

\section{Implications}

This chapter suggests, above all, that link citation practices are somewhat tacitly standardized on Slate and Newsweek, despite the fact that neither publication has formalized those practices. Most uses of hyperlinks fall within demonstrably typical bounds, and those that do not often appear to be making a rhetorical point via that unconventionality. The connection between link citation practices and circulation is, perhaps, the most surprising and salient finding of this chapter: A piece's frequency of links, especially when those links are used in particular ways, appears to be a factor in how a piece gets shared on social media. In that tacit standardization, this analysis points to some potential avenues of future research: Do other sites for short-form, networked web journalism share the same practices? Have these practices changed over time, and if so, how? Are they changing, or relatively stable-for-now?

Who else might be able to make use of these findings? People who write for the web or teach web writing may be able to make use of my cataloguing of link citation practices; this chapter's "rhetoric of hypermedia" combined with the more pragmatically oriented section at the close of this dissertation are intended both to make style recommendations for web writers and to provoke meaningful discussion in classes that study or require web writing. Discussion of citations in writing classrooms often tends toward the instrumental, sometimes presented punitively, rather than the rhetorical; 
students are given guides to the forms of citations and told that these rules, if broken, carry swift and devastating punishment. This study opens a conversation about the rhetoric of link citations that students could pick up in other work. Students could, as Lee and Swales (2006) describe, conduct smaller-scale but similar corpus investigations as part of a course. Web historians could open up this chapter's project diachronically, comparing the practices I have examined with practices in past or future publications. The notion of a link as a visual, material feature, emphasizing particular points, might also be useful for document designers.

More to the point of this dissertation overall, I hope that rhetoric and composition scholars might be able to operationalize some of my findings to direct their plans for entering online spaces. I hope that in the next year or so, I will be able to take some of these findings into a revision of the Kairos style guide; modeling link citation practices that improve Google search results and increase the chance of social sharing might raise the disciplinary standing of such journals. Ultimately, I argue that we need a new kind of research notes-style web publication aimed at a public audience. In service of that argument, this chapter also sets the stage for the investigations that make up the rest of this dissertation: In Chapter III, I pick up the question of how these link citation practices differ from traditional academic print parenthetical citation practices, specifically in $C C C$ and College English. In Chapter IV, I combine my findings from this chapter's analysis with my findings from Chapter III to offer insight into the citation practices of webtext journalism, which sits in a liminal space between popular web writing and the traditional academic print journal article. And finally, in Chapter V, I call for a new kind of publication, with a new style guide to kick it off. 


\section{CHAPTER III \\ "PHONY STORY": \\ CITATION MYTHBUSTING, DISCIPLINARY VALUES, AND MINING FOR \\ APORIAS \\ IN RHETORIC AND COMPOSITION}

'Preparing to write the next draft of the manuscript, Davis alluded to the 'phony story' that she would have to add to the Introduction. When pressed as to what she meant, she simply noted that 'reviewers always expect you to say certain things.' Davis' efforts to develop a 'phony story,' that is, to contextualize her experiments within a related literature, can be seen in Draft 4. [...] [S] he expanded the citational base of the Introduction."

- Carol Berkenkotter and Thomas N. Huckin, Genre Knowledge in Disciplinary Communication, p. 54-5

When I ask first-year students what they do when they first pick up a new book, they have lots of answers - read the back cover, skim the chapter titles, read the first page, read the last page_- but nobody ever answers like Sidney Dobrin. Dobrin (2002) claimed he prefers to flip to the back to read the references before reading any of the text: "Whenever I pick up a new article or book, I always look first at the Works Cited page and make some judgment about the value of the work, and I'm willing to bet this is a common way for academics to read, a common way for many of us to assess worth before we read the body of the text" (p. 55). Whether Dobrin is right about the commonness of this practice among academics in rhetoric and composition (my anecdotal evidence suggests otherwise), his comment makes this clear: Much is at stake in the way academics cite. Citation, particularly in a discipline's most important journals, can construct and maintain both disciplinary and authorial identities. It locates us and our 
work in the greater textual fabric of the field, and meanwhile helps make up that textual fabric.

Then, of course, citation also serves a variety of more specific contextual purposes, supporting ideas, linking them to other similar ideas, and establishing authority. Peter Elbow (1991) described the persuasive function of citation thus:

As academics, that is, we have various aids to authority. The most obvious one is to take a ride on the authority of others - and so (naming, finally, the most conspicuous stylistic convention in the genre) academics use footnotes and quote important figures in our writing. What we write is not just a neat idea we had that we send out to be judged on its own merits; it builds on Aristotle and echoes Foucault. [...T]he price we pay for a voice of authority is a style that excludes ordinary readers and often makes us sound like an insecure or guarded person showing off. (p. 148)

Here, Elbow echoes the biologist from the epigraph who described literature reviews as a "phony story": We build upon previous scholarship, sometimes aligning ourselves with it, sometimes setting ourselves against it. Citation is at once the obfuscating evil and the discipline-building keystone of academic writing. Academics reference Aristotle and Foucault even as we roll our eyes about feeling obligated to reference Aristotle and Foucault.

Indeed, among rhetoric and composition scholars, disciplinary narratives about citation, and the realities of our citation practices, tend to sit in this place of tension and ambivalence. Some of our narratives contradict one another. Other narratives are contradicted by our practices. This dissertation chapter, through distant reading (Moretti, 2005) of citations in College English (CE) and College Composition and Communication $(C C C)$, and through interviews with editors of those journals, seeks to bust some of our citational myths. As in the previous chapter, I seek evidence of tacit practices and the 
assumptions and values that are revealed within those practices. I highlight aporias in our citational narratives and between our narratives and practices; from these aporias, I suggest ways forward - not necessarily revisions of citation practices, which result from a complex pastiche of concerns local and global, but revisions of our narratives about citation practices, of the ways we teach citation, and of the ways we represent citation expectations in and journal guidelines and style guides.

To be clear, I do not intend this chapter as a finger-wagging exercise. In using the term “aporia," I mean to borrow from Jacques Derrida (1993), who explained aporia as a "nonpassage, or rather from the experience of the nonpassage, the experience of what happens and is fascinating in this nonpassage, paralyzing us in this separation in a way that is not necessarily negative: before a door, a threshold, a border, a line, or simply the edge or the approach of the other as such" (p. 12, emphasis mine). That is, in identifying disunion between values and practice, the natural implication seems to be that we must change either our values or our practices to match; yet, in the case of citations, massive disciplinary changes are neither easy nor guaranteed to make a positive difference. However, recognizing that our citational practices and values are often at odds should give us pause — we should dither at the threshold, maybe reconsider the ways we enter academic discourse. How is it, I mean to say, that we can hold simultaneously such competing narratives, and that our most foundational of writing practices can so wholly contradict some of our narratives? Thus, while I offer at the end of each section a series of recommendations for stakeholders in the field (students, faculty, editors of journals), I hope for these to serve as starting places rather than conclusions. I focus on aporias in two realms: 
1. Novices' Citational Lack vs. Excess. We hold simultaneous competing narratives about newcomers' citation practices: That on the one hand, they cite too much (and on average, my data supports the perception that newcomers cite more often). On the other hand, reviewers seem to express a sense that "novice" status is betrayed most obviously by a lack of citations - and, in fact, the mostestablished members of our discipline are the people most likely to cite “extremely," with articles that evidence either impressively high or surprisingly low citation count. These narratives reveal, behind their language of "lack" and "excess," a deeper and yet unarticulated concern about the correct kind of citation.

2. Slow Hegemonic Burn. Against the narrative of increasing disciplinary openness and explicit attention to race, I investigate the paracitational practice of eponymous adjective use, revealing a slow burn of academic hegemony that, through our assumptions about shared knowledge, reproduces biases and compounds the effects of underrepresentation.

Without distant reading, it is difficult to tell the difference between one's own sense of citational practices and the aggregate reality. Several times, I was surprised by my findings; my graphs told stories I did not expect to see. Thus, I wish also to make a parallel methodological argument for corpus analysis as a means of disciplinary narrative triangulation. When we write, read, and speak about how we do things, corpus analysis is an invaluable tool for cross-checking those assumptions. 


\section{Trend toward Hypercitation}

The field of rhetoric and composition has been tending increasingly toward higher and higher numbers of citations in our major journals, according to Derek Mueller's (2012) analysis; from 1987-1991, there were 2755 works cited in College Composition and Communication (CCC), compared to 4957 works cited from 2007-2011 (p. 204). ${ }^{24}$ Rhetoric and composition was built first on the backs of a small handful of journals, which became academic journals (and not simply newsletters) when they developed peer review processes and began to demand that authors cite research. $C C C$, for instance, predates the first grantings of rhetoric and composition PhDs by more than three decades. Since it became the flagship journal of a burgeoning discipline, $C C C$ has demanded its authors demonstrate increasing referentiality to other work in rhetoric and composition. Joseph Harris (1997) explained this shift from personal narrative to so-called "serious research":

There are now books and articles to read and cite, canonical figures to contend with, in writing about almost any aspect of composition theory or teaching. [...] Or to put it another way, the members of a field long associated (both in the classroom and out) with the writing of personal essays now find themselves answering a demand for research articles. (p. 47-8)

Indeed, this shift has been quantitatively documented. Between 1987 and 2011, the average number of cited works per year of $C C C$ 's publication rose from about 400 to about 1000 (Mueller, 2012, p. 199). Furthermore, as the field has professionalized,

\footnotetext{
${ }^{24}$ Mueller's measure of the rise in cited works is perhaps not the ideal measure - citations-per-word or per-paragraph might be more useful, given that numbers of articles change over time. I have calculated mean words per citation in my corpus overall: In College English, there is a mean of 144 words per citation, or 0.8 citations per paragraph; In $C C C$, there is a mean of 158 words per citation, or 0.72 citations per paragraph. I have the data to make a revision of Mueller's metric, charting change in citation rates over time, but I did not have the time; should I publish this research, I will make that comparison.
} 
Mueller's (2012) analysis suggested, it has also become broader, less centralized, and less dominated by superstar scholars and publications - all evident in its citation practices.

In a historiographic look at the rise of academic journals in rhetoric and composition, Maureen Daly Goggin (2009) argued, "Academic journals stand in a dialectical relation with a discipline, both responding to disciplinary practices already in place and fostering (or authorizing) particular kinds of practices by accepting some and rejecting others. [...] As such, journals serve as an important locus of disciplinary power, shaping a discipline even as they are shaped by it.” Thus, she said, we can learn much about the power machinations and shaping forces of a discipline by studying the way its key journals construct and are constructed by disciplinarity (p. 324). I seek to draw out the dialectic that Goggin suggests sits implicitly between academic journals and the disciplines they construct. Hence, I turn to College English and CCC to understand something both about our disciplinary values and the practices by which we write and publish.

\section{Aporia 1: Newcomer Excess vs. Lack}

We hold simultaneous competing disciplinary narratives about how newcomers' citation practices: On the one hand, both Kelly Ritter and Jonathan Alexander expressed their concern that newcomers are more likely to "over-cite"; Ritter was especially vehement about this concern, explaining that she often has to reassure graduate students that they do not need to "read everything" before they can write. On the other hand, reviewers express a sense that they can recognize "novice" writers by a lack of citations. When compared with the data, this aporia makes some sense. Newcomers do indeed cite, on average, more often than the authors on Mueller's "most-cited" list. However, the 
"most-cited" authors actually have the most disproportionately excessive and infrequent citation practices; they are overrepresented at both the high-citing and low-citing ends of the spectrum. And newcomers are less likely than other groups to use more than one citation per parenthetical statement (i.e. use a "citation string"), a common practice that some (including Ritter and other unnamed journal editors with whom she's discussed the issue) believe to be associated with newcomer citation practices.

From this aporia, I suggest that we ought to reconsider our terms: Our tendency to discuss newcomer citation in terms of "excess" and "lack" is insufficient for describing the legitimate concerns editors and reviewers express. While there are indeed some demonstrable differences between citation practices of newcomers and of people who are more established in the field, broad admonitions against "citing too much" or "not citing enough" do little to ease enculturation of newcomers. My analysis stays primarily focused on the realm of citational quantity — my methods are particularly well-suited to answering those quantitative research questions. But I point to a need for future research in the qualitative distinctions between "newcomer" and "expert" citations. For instance, what are the kinds of habits and attitudes and practices that make it possible for a wellestablished author to publish a 0 -citation article or a 150 -citation article, without being read as "lacking" or "excessive," respectively? What is it about a middle-of-the-road 70citation article by an "Emerging Voices" author that might be simultaneously read as citationally lacking and citationally excessive?

\section{Maybe "Junior Folks" Cite Too Much}

One disciplinary narrative suggests newcomers cite "too much"—and this narrative, at least, seems supported by data. Compared to the journal-wide averages, in 
both $C C C$ and $C E$, the authors on Mueller's list skew toward the low end of in-text citation counts per article, while authors who have only been published once skew toward the high end (see Figure 3.1). 


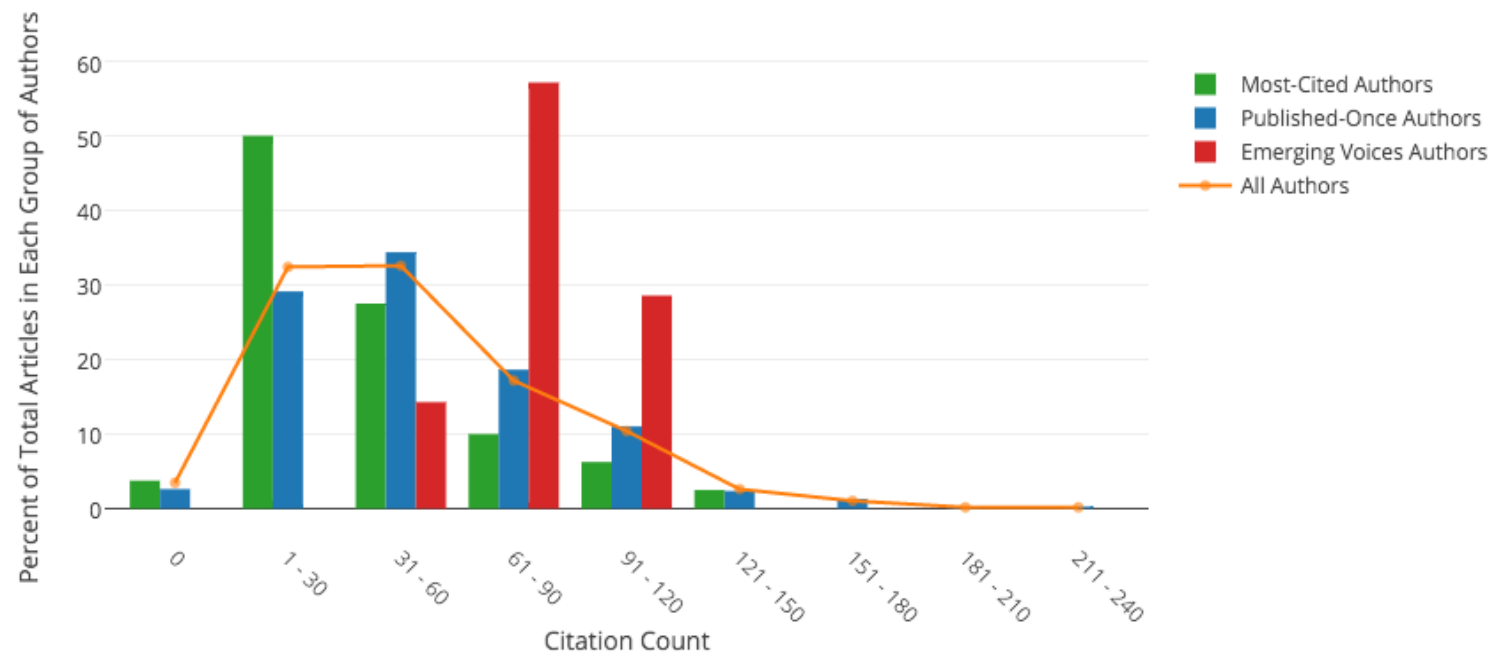

CCC: Percent of Articles in Each Group of Authors by Citation Count

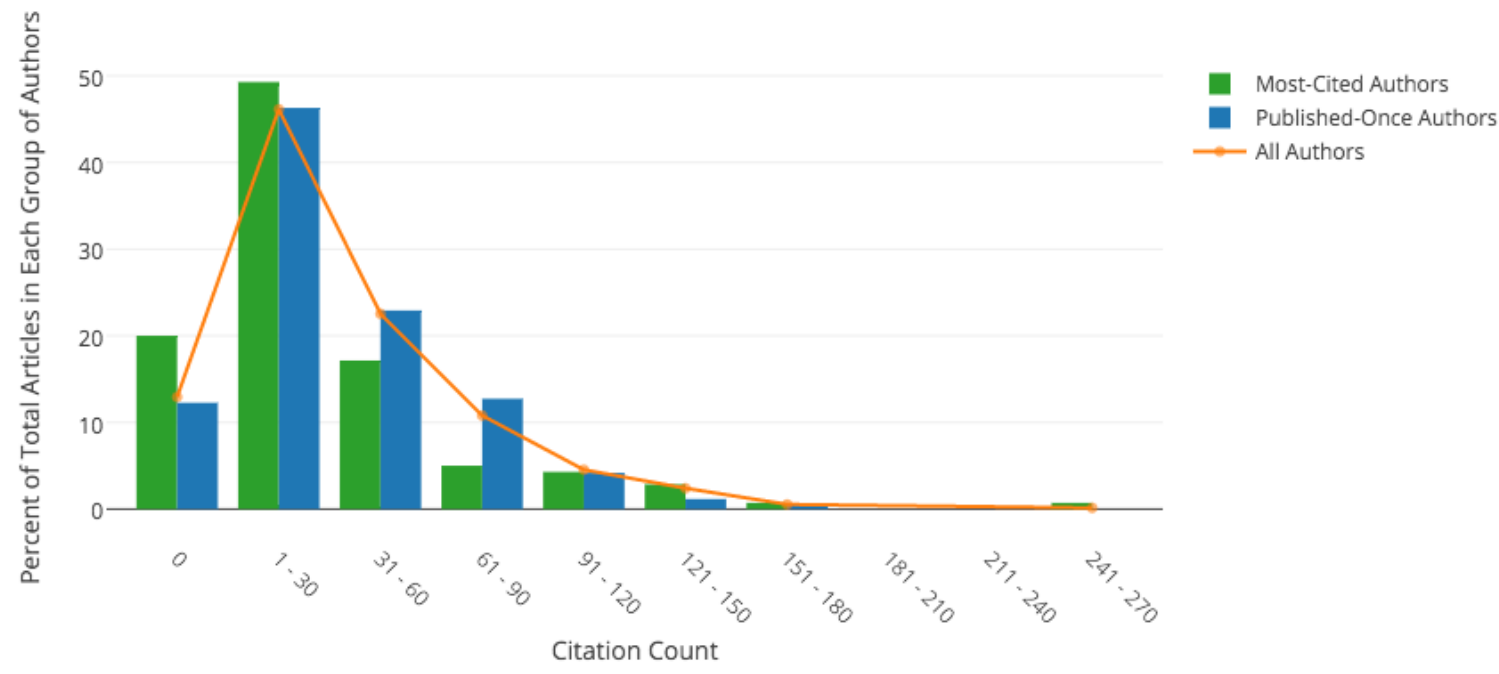

Figure 3.1: The percentage of articles per citation count, in $C E$ and $C C C$. Note the leftskewness of most-cited authors (slight in $C C C$, more profound in $C E$ ), the slight rightskewness of published-once authors, and the extreme right-skewness of "Emerging Voices" authors. 
Most-cited authors top both charts through 30 citations per article; for instance, $69.3 \%$ of articles by most-cited authors in $C C C$ have 30 citations or fewer, compared to $59.1 \%$ overall. In fact, in $C C C$, fully $20 \%$ of the articles written by most-cited authors have 0 total in-text citations - a particularly remarkable finding when recalling that this corpus does not include reviews, comments, and responses. Above 30 citations per article, in both journals, authors who have only been published once take the lead. Authors who have been published only once for the most part track close to the journal-wide average, which is noted by the orange line on the graph, in both journals; in $C E$, authors published once diverge from the average just twice, with a below-average percentage of articles with 1-30 citations and a slightly above-average percentage of articles with 30-60 citations.

The most dramatically right-skewed category is $C E$ 's "Emerging Voices," a group of authors identified as newcomers to the field. In September 2011, the $C E$ front matter began to invite first-time authors to self-identify upon submission, to be considered for a feature. This language quietly entered the front matter without much fanfare, just as John Schilb was passing editorship to Kelly Ritter; her first "From the Editor" section explained that the section was intended "to better help all of you to see yourself as potentially being part of this journal's future tables of contents" (p. 11). In an interview, she suggested the section was also intended to address complaints that the journal was not welcoming enough to new authors. When an article is so featured, the words "Emerging Voices" are added to the beginning of the article's title. Ritter said that she also tends to work more closely with these authors, with a willingness to go through more revisions. In my corpus, there are just seven "Emerging Voices" texts; yet four of those seven have 
between 61 and 90 citations, well above the journal-wide mean of 49 citations per article. And not one of the seven has fewer than 31 citations - unlike the vast majority of articles in the journal.

What might account for this hypercitational tendency of newcomers? Several editors of major journals link being able to break citation conventions with experience or authority in the field; conversely, novice status, they suggest, is tied to a sense that citation is a necessary means of proving oneself. When I interviewed Ritter, she described a recent experience submitting an archival research piece to a journal, an article that was more lightly documented than similar kinds of work she had done previously:

I had a little bit of pause where I thought, 'Oh, I wonder if reviewers will ding me for not citing enough people.' But when I was making my argument, I realized, I don't need those people, because this is a very unique situation in this archive, and I've established what I'm doing. Take it or leave it, right? But part of that might also be my lack of fear, because I've done archival work before and I've had it published; you either want what Kelly's selling or you don't. So I didn't really care. If the reviews come back and say, 'Hey, you didn't cite ding, ding, and ding,' I can stick them in. I think junior folks, logically, have the opposite view. [...] Often their response is, 'I've got to read everything before I start writing.' Every time a graduate student says that to me, I'm like, 'Oh my gosh, that's completely wrong.' You've got to write into your subject; you can't read everything. (emphasis mine)

The power of having published several similar pieces before, of being an editor of a major journal, of doing kinds of work she knows earn disciplinary respect, give her the confidence to present her findings with conviction, without relying on much citational authority to support her argument. Several times, she emphasized again to me the importance of making strong claims over citing heavily.

Though Ritter expressed this view to me after having heard about the results of my analysis, it is certainly not a new perspective. In his closing "From the Editor" 
column, John Schilb (2012) identified hypercitation as a troubling trend, particularly

common among the fresh-off-the-dissertation-boat crowd:

Naturally, indicating the main claim's import often entails relating it to previous scholarship. Indeed, authors may wind up delaying the claim because they feel they must review first everything published on their subject. This sense of duty is understandable - especially in the case of writers submitting parts of their dissertation, a genre historically required to survey established authorities. ${ }^{25}$ Still, many of the submissions we receive waste time by quoting or by otherwise citing everyone who's ever addressed their topic. [...] I may get in trouble for saying this, but here goes: rarely are scholars so eloquent that their own words must be repeated. (p. 214, emphasis mine)

Schilb drew the deliberative conclusion Ritter only implied: not only are newcomers citing a lot, they are citing too often and should cite less. Intriguingly, like Ritter, he suggests that he personally has transcended the "sense of duty" that plagues recent dissertators; his comment that he "may get in trouble" is surely sardonic, if I may risk pointing out the obvious - it is difficult to imagine how the outgoing editor of $C E$ would "get in trouble" for such a banal comment, or indeed what "trouble" might look like for John Schilb in 2012.

\section{Lack: If They Don't Cite Enough, They're Newcomers}

This recognition of average citational excess among newcomers seems to compete with the simultaneously circulating narrative that newcomers do not cite enough. When Paul Kei Matsuda and Christine M. Tardy (2007) interviewed two journal board members about their blind review of a submission to a major rhetoric and composition journal, both reviewers expressed confidence that the writer was a novice. The reviewers cited the writer's minimal use of references as a key marker of novice status: one suggested the

\footnotetext{
${ }^{25}$ There is irony, I am aware, in expressing the idea that newcomers cite too much with a big long series of block quotes. But Schilb says this genre calls for it.
} 
writer "just doesn't quite have enough of a bibliographic base" to approach the topic like an expert, and the other criticized the writer for a "dearth of references to [topic X] within rhetoric and composition" (p. 244). A lack of references, that is, is enough to suggest to some reviewers that an author is not central to the field. Reviewers here do not name a particular number of references that would be an adequate "bibliographic base," of course, or how many "references to [topic X]" would amend the "dearth." My method of analysis perhaps eclipses some potential truth behind this competing narrative: It is possible that newcomer writing that evidences such a "dearth of references" simply gets rejected out of hand, or pushed through the revise-and-resubmit process until it is deemed adequately citational. Yet I suspect that Matsuda and Tardy's reviewers' comments have less to do with the raw numbers of citations in newcomers' texts and more to do with a differential sense of centrality betrayed by the ways newcomers use citations.

What might account for this differential sense of centrality? My data are inadequate to answer this question, but I have some guesses: Maybe newcomers are more likely to over-explain and over-rely on works that might widely be assumed as "shared knowledge" among disciplinary insiders (e.g. Who among rhetoric and compositionists, for instance, needs a glossing of Bakhtinian heteroglossia? See "Aporia 2" for more on this). Maybe newcomers are more likely to cite the things they have read in graduate coursework, works that tend to be older, maybe the "foundational" texts of our field, even when/where those notions have become dispreferred (e.g. Citing cognitivist research like Flower and Hayes [1981] without adequate apparent recognition of its outdatedness). Or, conversely, perhaps newcomers are more likely to demonstrate too-few nods to that foundational history: Ritter suggested that newcomers might be more likely to cite in 
"trendy" subfields—she named "transfer" and "social media writing"—without enough recognition of how those subfields grew out of the longer history of our field. Without a more comprehensive qualitative content analysis, I cannot say for sure - but the aporia suggests that the notions of "excess" and "lack" are red herrings for deeper reviewer and editorial concerns about what kinds of citations are adequate to demonstrate insider status.

\section{The [Semi-Mythical] Newcomer Trainwreck Pile-Up}

Though newcomers do indeed cite more often than more-established authors, they do not participate as heavily in one practice that Ritter named as particularly troublesome: the citation string. ${ }^{26}$ Before I shared my data with Ritter, I asked her whether she noticed any differences between how newcomers cite and how moreestablished authors cite. "Yeah," she said, "in fact, I do. And this has become something that's been a real concern of mine":

What I see now, especially with junior folks, especially with graduate students, is what I call the 'trainwreck pile-up citation method.' And it goes like this: 'Scholars have been concerned about assessment across many different projects (name, name, name, name, name). Also, people think about transfer in this way (name, name, name, name, name).' [...] I've said in some of the letters to them, 'Don't do this. I want you to pull back on this some.' [...] What it does is it forces the reader to go into the Works Cited and go, 'Oh. You meant Susan Miller's book, Assuming the Positions. I guess that book in general must say what you're saying.' It's this macro-level engagement with other scholars that completely eliminates the micro, which I just find kind of troubling. [...] What it also might communicate to some readers, but it totally depends on the article and the topic and the ethos of the writer is, you may or may not have actually read. [...] Maybe this is just my generational preference for something that is akin to close reading,

\footnotetext{
26 "Citation string" is apparently a primarily legal term, for citing several cases in a parenthetical; the most widely used American legal citation system, The Bluebook, gives specific guidelines for the order in which authorities are to be listed in the string. I have not found any rhetoric and composition scholarship discussing the idea, though I have anecdotal evidence that the term is in circulation in our discipline: I first encountered the term in Karen Kopelson's "Composition Theory" graduate seminar, where we discussed its prevalence in research introductions.
} 
although I don't think it's close reading. But I think it can diminish the ethos of the author, potentially a lot, depending on what they're actually trying to string into the cites.

Ritter voiced here three related concerns: As a field, we are using more citation strings ${ }^{27}$ than in the past. Newcomers use more citation strings than more-established people. Citation strings indicate lower engagement with the source text, perhaps to the point of suggesting that the author may not have even read the source text.

I graphed the percentage of total parentheticals per year that include a reference to more than one work (see Figure 2), from 1994 - 2014 in $C E$ and from 1997 - 2015 in $C C C$. In $C C C$, the citation string usage graph reveals a slight upward trend, with a peak just under $10 \%$ in 2015. Citation string usage in $C E$ has been more variable (with a peak of about $9.4 \%$ in 2001), but the trend line has remained relatively flat. That is, as a field, we are perhaps using slightly more citation strings than we were twenty years ago, though neither dramatically nor consistently. The year Kelly Ritter took over CE in 2012, there was a large drop in the percent of CE parentheticals that were citation strings, from nearly $8 \%$ to just over $4 \%$, followed by a return the next year to normal levels - perhaps this is the "rise" she saw, perhaps her efforts to get authors using fewer citation strings were more zealous/effective early on, or perhaps there is some other factor in play. Further, the upward trend in $C C C$ citation string usage is interesting, compared to the relative flatness of $C E$ 's; perhaps this difference is attributable to College English's more literature- and creative writing-influenced bent and $C C C$ 's increasingly social scienceinfluenced practices - though, of course, that is merely speculation.

${ }^{27}$ As colorful and lovely as "trainwreck pile-up" may be, I'll here prefer the more neutral term. 
CCC and CE: What percent of parenthetical citations each year are citation strings?

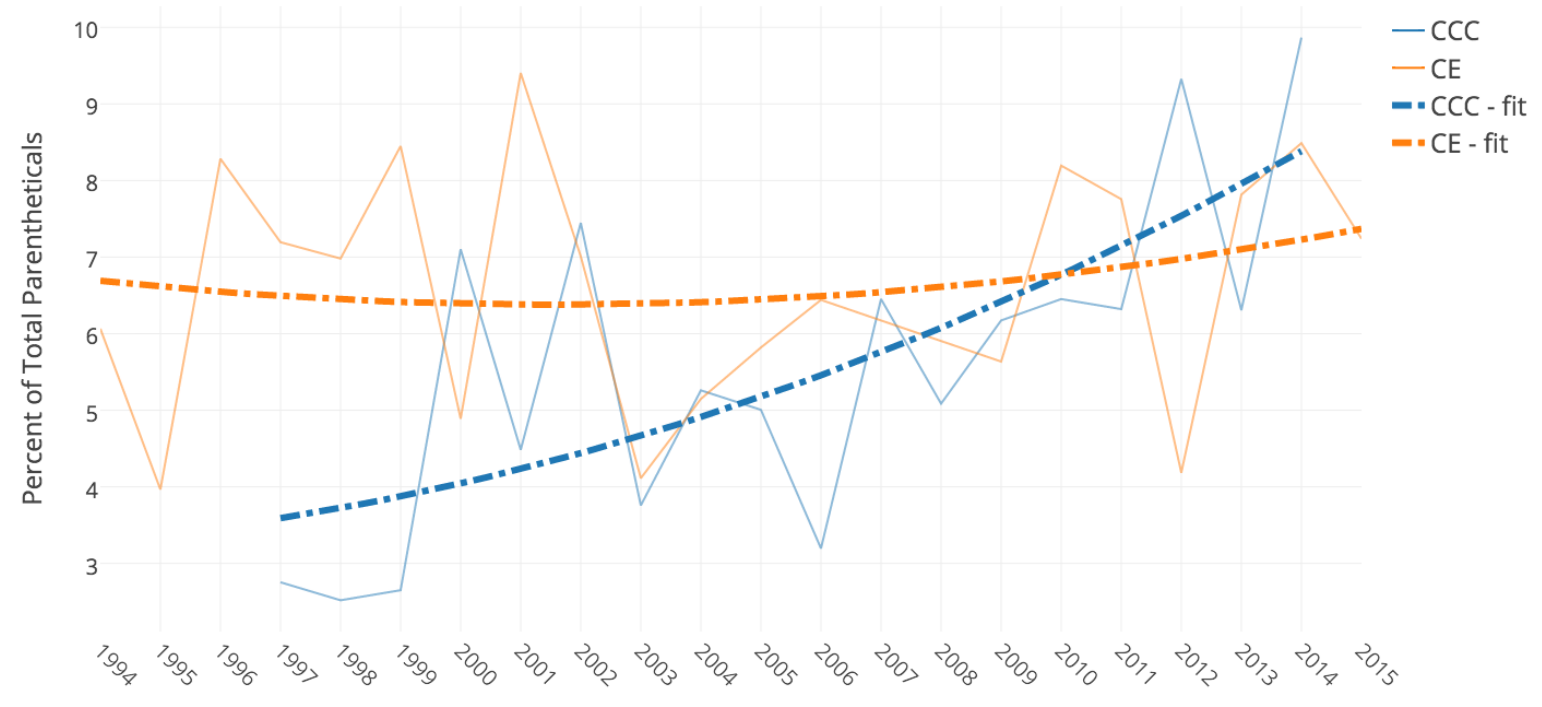

Figure 3.2: Never have citation strings accounted for more than $10 \%$ of all parenthetical citations in either $C C C$ or $C E$; the quadratic fit trend of citation string usage in $C E$ has remained fairly flat. However, in $C C C$ there is a pronounced upward trend in citation string usage.

Fairly unequivocally, however, newcomers do not use more citation strings than mostcited authors (see Figure 3.3). Most-cited authors in every case use more citation strings than other groups; in fact, more than $10 \%$ of most-cited authors' parenthetical citations, in both $C E$ and $C C C$, reference more than one text. Authors published once use about an average percentage of citation strings. Emerging Voices authors in $C E$ use a lower-thanaverage percentage of citation strings; just 2.1\% of Emerging Voices authors' parenthetical citations are citation strings. 
CE: How common are citation strings, by group?

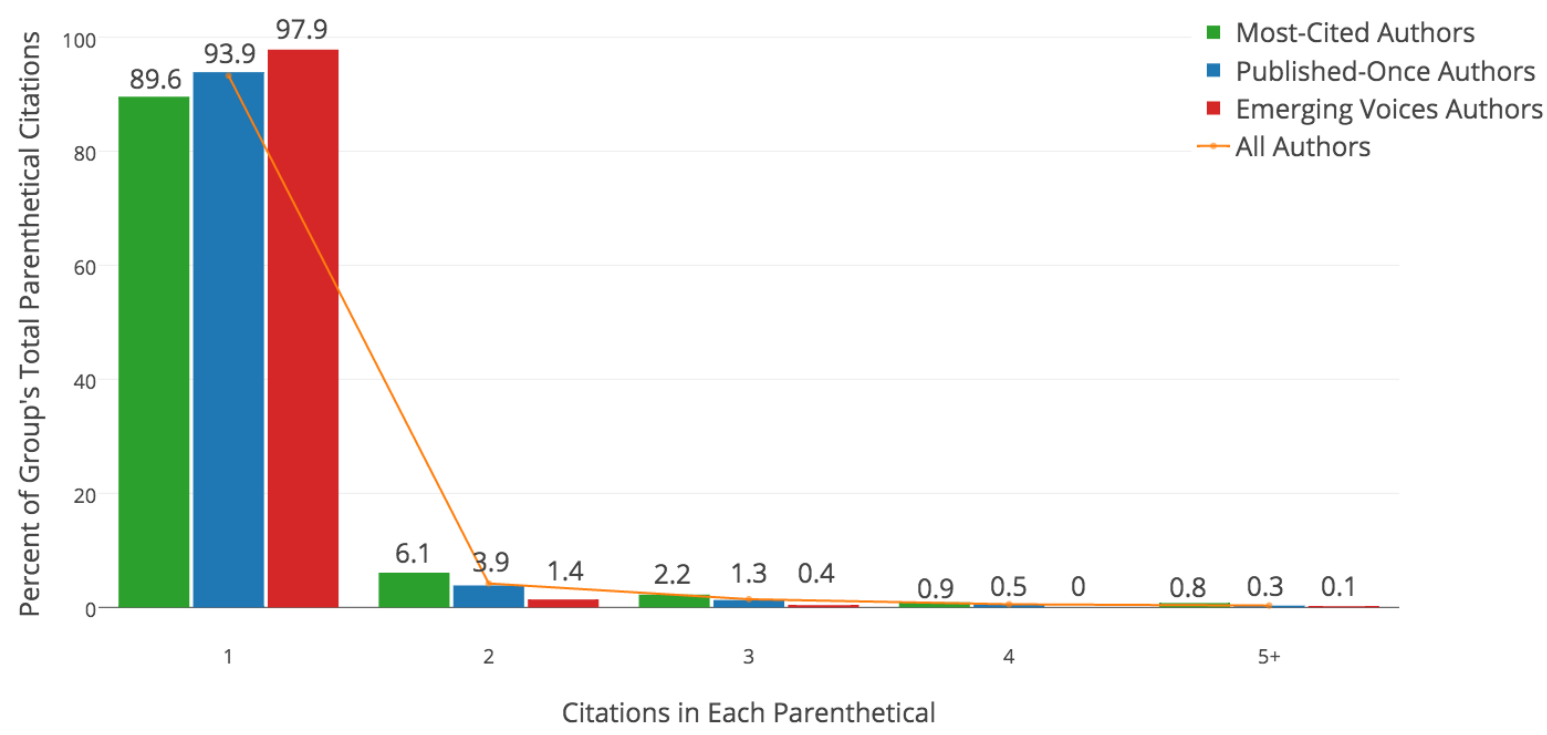

CCC: How common are citation strings, by group?

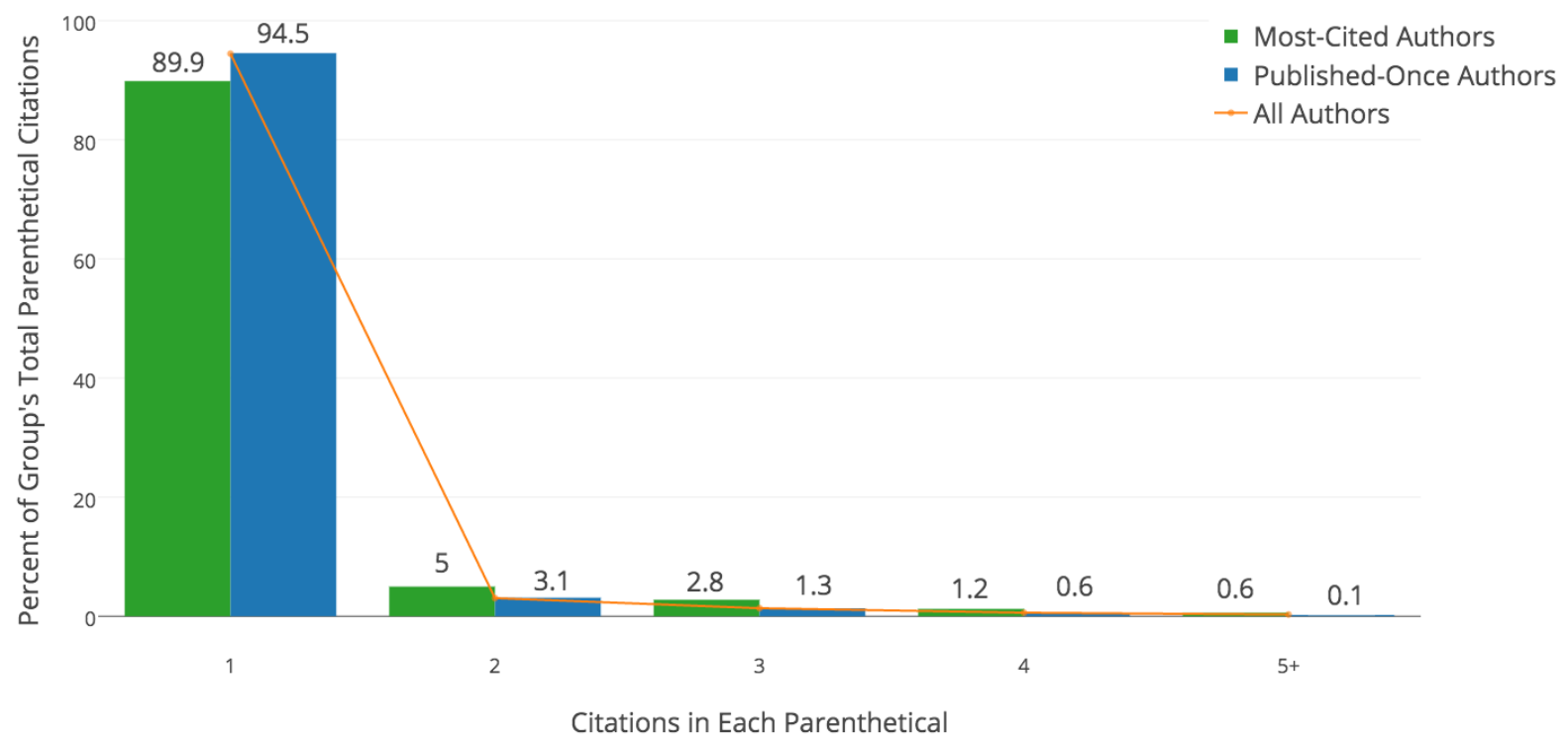

Figure 3.3: In every single case, both in $C E$ and $C C C$, the most-cited authors have a higher proportion of every type of citation strings and a lower proportion of parentheticals with just one citation.

Again, this suggests that the narratives of excess and lack are deceptive short-cuts for other, deeper concerns about citation practices: If newcomers are absolutely not using 
more citation strings than most-cited authors, and yet Ritter and Alexander see newcomer citation as characterized by over-use of citation strings, perhaps there is something about the way newcomers are using those citation strings that calls readerly attention to them. This points to interesting questions for future research: Might, for instance, newcomer citation strings group authors whose work is normally not grouped? Might the citation strings that newcomers use include out-of-date work? Or, conversely, might the strings too-casually gloss obtrusively bleeding-edge work that really demands more engagement? Might the phrases attached to the citation string suggest a misunderstanding or mischaracterization of the works represented?

\section{Excess: The "Most-Cited" Use Citations More Extremely}

The recognition that the most-cited authors use citation strings much more often than newcomers accords with another finding: Most-cited authors cite more extremely, at both the high and low end of citation counts. Figure 3.4 demonstrates the proportional representation of most-cited authors at different citation counts; each bar represents a group of citation counts per article and displays the proportion of those articles written by most-cited authors, as a percentage difference from the average proportion $(16.4 \%$ of all articles). 


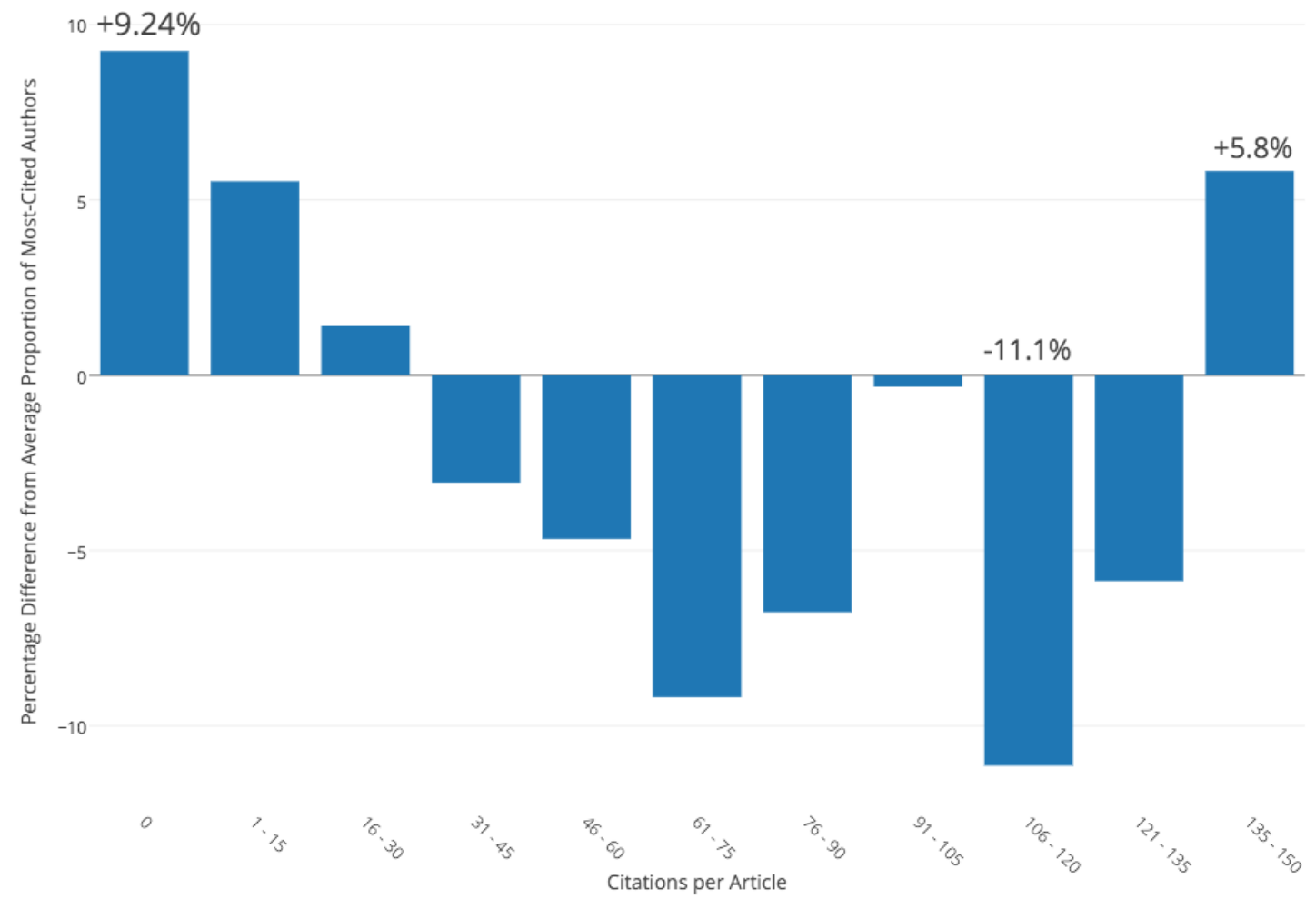

Figure 3.4: The percentage difference from the average proportion of most-cited authors at different citation count buckets. At 0 citations per article, most-cited authors account for $9.2 \%$ more articles than they represent in the corpus overall. At 120 citations, they account for $11.1 \%$ fewer articles than they represent in the corpus overall.

I intend to call attention to the slope of this data: At the low end of citation counts-from 0 through 30 citations - most-cited authors are significantly overrepresented. This overrepresentation is the strongest at 0 citations per article; this finding accords with my experience, in that it would seem hubristic indeed for someone in my disciplinary position to submit an article to $C C C$ that cited no other articles. If I was lucky enough to get feedback from reviewers, I could expect them to ask, "Where is this work located in the field?" Most-cited authors are also significantly overrepresented at the highest end of 
citation counts, from 135-150 citations per article. This finding, too, makes some sense; the time and disciplinary resources that go into doing the kind of archival work that primarily makes up that highest-count bracket is largely out of the reach of the newest scholars. In the middle, however, from 31-135 citations per article, most-cited authors account for a disproportionately low number of those articles. An article with 106-120 citations is $11.1 \%$ less likely to be written by a most-cited author than average. Thus, while most-cited authors cite less on average overall, when their work is bucketed by citation count, it becomes clear that they cite "excessively" at both ends of the spectrum: They are far more likely than average to have very-high-count and very-low-count articles, and less likely than average to have middle-count articles.

Notably, particular "most-cited" authors account for a large proportion of those extreme articles. An overwhelming majority $-94.8 \%$ - of articles on $C C C$ and $C E$ combined have 100 or fewer citations (see Figure 3.5). That is, just 69 of the 1,327 articles in my corpus have more than 100 citations. Of those 69 articles, five are by one author on the "most-cited" list: Bruce Horner alone accounts for $7.25 \%$ of the articles with a citation count above 100. This tendency toward hypercitation does appear to be a habitual authorial trait for some authors. Six other authors have more than one article on the over-100 list: Suzanne Bordelon with 3, and Joseph Janangelo, Karen Kopelson, Laurie Grobman, Jessica Enoch, and Peter Mortensen with 2 apiece. Kopelson's inclusion on this list did not surprise me, for what it is worth, given that I used her (2015) recent $C E$ article as a test case when writing my "janky JSTOR." 


\section{CCC and CE: Citations Per Article}

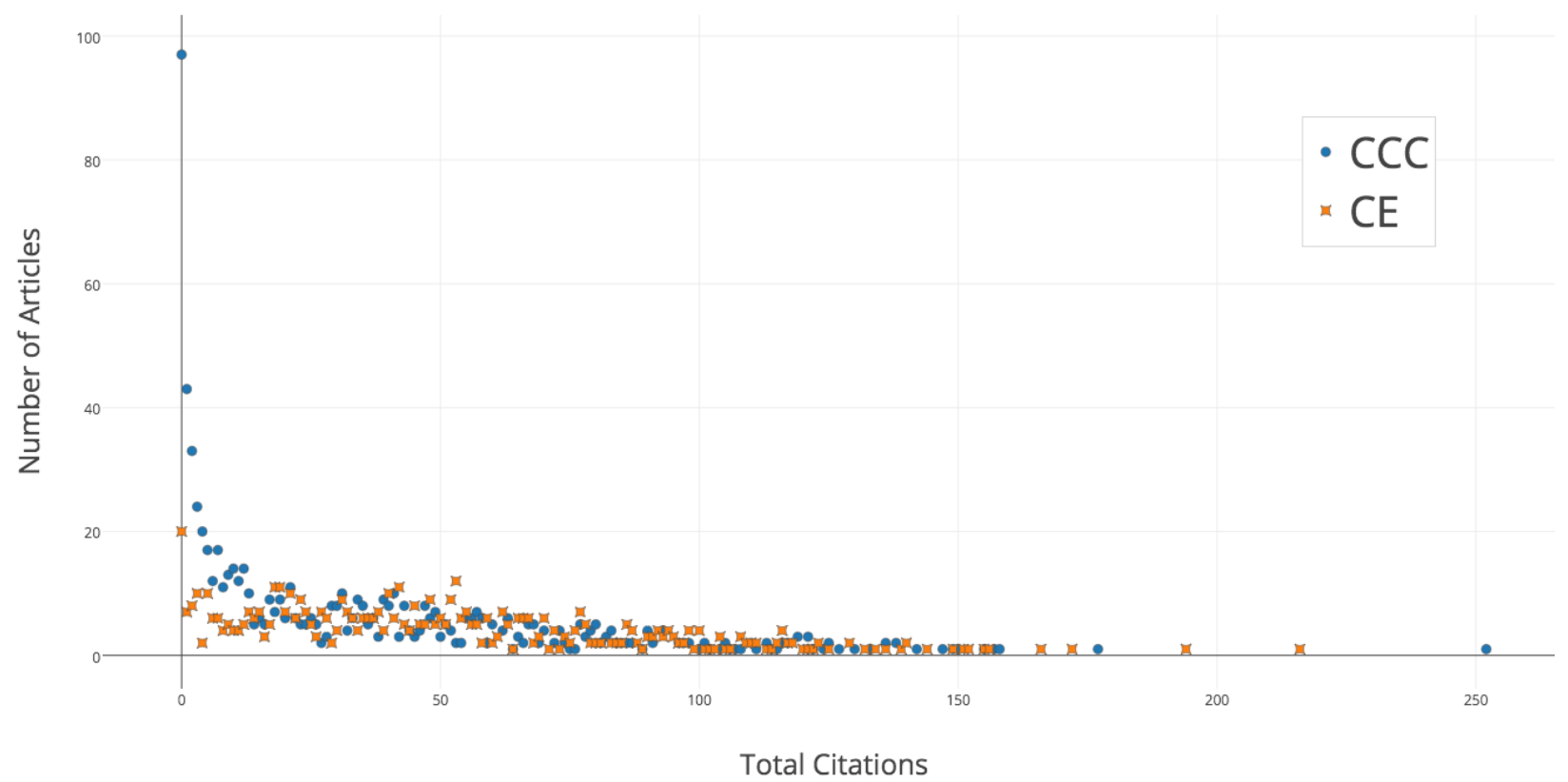

Figure 3.5: The number of articles with a given citation count, in $C C C$ and $C E$. Note the leftskewness of both series, particularly $C C C$, and the long tail to the right. Only 69 articles across both journals have greater than 100 citations.

Some authors, curiously, end up on both the high and low end of the spectrum:

Ritter herself has the seventh-highest number of total in-text citations in $C C C$, at 262 intext citations across her three publications in the journal (when I told her this, she said, “Gosh, 262. How did I fit all those in there?”). In her one non-editorial publication in my corpus in $C E$, however, she has just 13 citations; this citation count ranks her at 487 out of 571 authors in $C E$. This distinction is explicable by differential subject matter: in her $C C C$ pieces, she explained, she was working with archival materials, which meant a lot of direct engagement with printed source material. Indeed, after offering this explanation, Ritter identified of her own accord two of the six people with more $C C C$ in-text citations 
than herself, Jessica Enoch (with 272 citations) and Suzanne Bordelon (with 502 citations), both of whom also do primarily archival work.

Ritter's $C E$ publication, however, was about doctoral-level creative writing studies - and, she explained, "People who do creative writing studies, as one of them joked to me once, can all fit into one hotel room." Much of her material came from personal interviews and course documents. Notably, that article was Ritter's first publication in writing studies; the natural limit to available citable material can perhaps explain how she was able to flout citation convention even without established disciplinary authority.

Sidney Dobrin (2002) argued that his "position as professor, published writer, compositionist allows [him] to take such chances" as making relatively minor changes to citational practices (p. 55). As a thought experiment, he proposed what might have happened had he submitted his Alt/Dis: Alternate Discourses and the Academy chapter with the Works Cited page before the text:

If, in this collection, I had explained my textual adjustment, it may have been left as is as an example of 'alternative,' as a trite, token example. [...] But what if a student had used a similar strategy, even explained it? Chances are the reactions would be less accepting. Chances are, this thing we've called academic discourse would swallow up, silence the student's parent discourse, her voice, her power. (p. 55)

Newcomers to the field are not all still students, but they are all closer to student status than someone like Dobrin. Establishment in a field gives authors the freedom to operate on an assumption of intentionality. Whether their citation counts are abnormally high or abnormally low, authors who are well-known are surely less likely to encounter an 
editorial challenge (and, probably, to perceive such a challenge as a threat) than their newcomer counterparts.

\section{What to Make of the Excess vs. Lack Aporia?}

The excess/lack aporia reveals a widespread shared sense among rhetoric and composition disciplinary insiders that something is wrong with the way newcomers cite, something at least in part quantitatively definable. Yet although newcomers do cite more often, on average, and although I have pointed to ways that more-central scholars are actually more "excessive" in their citation practices, I do not mean to suggest that my quantitative data should dismiss this excess/lack concern: Indeed, if insiders universally feel that newcomers are citing "wrong," it suggests that we should indeed be paying more attention to disciplinary citation practices - in graduate education, in our journal guidelines, and in our scholarship. The conflicting narratives and diversity of definitions of "excess" and "lack" also suggests that these categories are likely inadequate to describe "wrong" citation.

Clearly, citation is not just a matter of numbers. People do not, of course, make tally marks on the side of the page as they read, counting up the numbers of in-text citations and comparing that number against some kind of quota. In fact, my data indicate that readers' sense of citational patterns is fairly disconnected from the aggregate reality, on average; even editors who read huge numbers of incoming articles may believe they are seeing trends that are not borne out by the data (such as in the case of citation strings). Rather, the senses of both newcomer excess and lack likely come from context: In some situations, newcomers likely do cite too little — they do not show evidence of awareness of the core texts in a given line of thinking, rehashing ideas that have already been 
established, presenting given information as if it was new, relying too heavily on individual source texts. In other situations, newcomers likely do cite too much-they tend toward enormous chunks of unnecessary literature review, they "perform student" and feel obligated to demonstrate having read, they lean too heavily on block quotes or lists of scholars who have addressed a well-trodden topic, they cite disciplinary common knowledge, they quote core texts that don't need to be quoted. My research points to the need for a fuller categorization of these practices, a deeper investigation of what citation practices are read as "too grad studenty" and what practices seem to evidence the steady hand of a most-cited author. By making our disciplinary expectations about citations more explicit, we stand to gain greater disciplinary openness, to newcomers of all stripes.

Furthermore, the finding that most-cited authors skew toward the high and low ends of citation counts may be reflective of genre- or project-based practices. For example, maybe most-cited authors are more likely to tend toward heavily-citing forms such as archival research because they have the time, funding, and training to spend several years on a project; maybe they are more likely to tend toward infrequently citing forms such as classroom narratives, because they do not risk disciplinary status by doing so. Without a more detailed content-based analysis, I can only guess.

My data demonstrate that there is indeed a quantifiable difference in how newcomers and experts cite, and that difference defies our expectations about how newcomers/experts cite. They make clear that the lack/excess many reviewers and editors see is not necessarily based in numerical reality. Thus, discouraging newcomers from citation strings because most-central scholars do not use them is disingenuous; mostcentral scholars use them more often than newcomers. Rather, citation string advice (as 
all citational advice) should be contextual: Is this a conversation that needs to be glossed or rehashed? Would any of your audience invoked be unfamiliar with these authors? Would someone new to the subfield be aided by this gloss? Again, newcomers may not always be able to answer such questions, by virtue of their newness, but such queries would likely prove a more useful heuristic than "don’t feel obligated to cite everything you've read."

When I take up this data in a future publication, I would like to consider the genres of citing texts, to understand whether some of the citational patterns of newcomers and most-cited authors can be explained by generic differences, as well as to understand generic citation patterns more broadly. I would also like to consider the ages of works cited, particularly in citation strings: Are people more likely to string recent works, to situate themselves in terms of a current conversation? Or do they string older works, to avoid having to rehash already well-established ideas? Do these patterns differ between newcomers and most-cited authors? Such analyses might help me bridge the gap between the citation patterns I have found and the content-related citational concerns my interviewees expressed.

From the disciplinary impasse I have noted, I call for greater attention to the whys and hows of our citation practices, attention both in our scholarship and in our graduate education. In our research, I hope for more mixed-methods explorations of citation, to help make the tacit explicit. In our graduate education, I suggest more heuristic-oriented citational advice. I recommend, too, that graduate studies include both analysis and production of genres with a wide variety of citation counts: Understanding how citation patterns differ among classroom narratives and archival work and longitudinal 
ethnographic research (and so on) may help newcomers approach citation with a more contextual eye. And when we all talk more about why we cite the way we do, perhaps we will also teach it more contextually in undergraduate classes.

\section{Aporia 2: The Slow Burn of Paracitational Homogeneity vs. Disciplinary Colorblindness}

Almost twenty years ago, Catherine Prendergast (1998) chastised rhetoric and composition for failing to consider race in explicit terms, even/especially where race would have been a meaningful analytic category. This disciplinary colorblindness, which rolls race into categories such as college preparedness and class, downplays the importance of race as an axis along which values and practices get aligned. Prendergast directed our attention to the work of critical race theorists, calling upon rhetoric and composition scholars not just to acknowledge "that racism is one of those scenic unconscious practices" but also "to identify and expose it not as an aberration, but as business as usual in the United States" (p. 37). Prendergast pointed to Keith Gilyard's (1996) unpublished Watson Conference paper, which proposed that "race remains undertheorized, unproblematized, and underinvestigated in composition research leaving us with no means to confront the racialized atmosphere of the university and no way to account for the impact of the persistence of prejudice on writers and texts" (p. 36). In the time since, many have joined Gilyard in turning a race-conscious eye back on our field (e.g. Jacqueline Jones Royster, 1999; Banks, 2005, 2011; Ballif, Davis, and Mountford, 2008; Kynard and Eddy, 2009- to name just a few). Yet change in our practices has

come slowly. In this year's Watson Symposium and Conference, Carmen Kynard (2016) pointed to ways that the academy has continued to reproduce the "vulgar essentialist 
claim, [that] 'neoliberalist multiculturalism,' or 'empty pluralism'/diversity that would assert that more black female bodies in classrooms and research will alone change the academy" (p. 20). We must, that is, continue to keep race as an explicit analytic category and continue to look for ways that our "scenic unconscious practices" reproduce biases inherent in the academy.

I wish in this section to use distant reading of one paracitational practiceeponymous adjective use - to consider the ways that the historical demographic boundaries the academy has drawn continue to reveal themselves in our most basic textual practices. Specifically, I am looking at eponymous adjective use as a telling academic practice because it exposes assumptions about shared knowledge in a way that more common citation practices cannot, assumptions that reproduce biases endemic to the academy. Eponymous adjective use appears to be in (unsteady, qualified) decline, over the last 20 years; yet, it persists, and it represents just one yet-unanalyzed example of the ways our practices can compound underrepresentation. Although eponymous adjective use is by no means unique to rhetoric and composition, I argue for the value of calling attention to how this textual practice reproduces a homogeneous view of academic knowledge — a white, male, Western, high theory academic history that is "business as usual in the United States," from which we imagine ourselves increasingly distant, and that nevertheless bubbles up into texts in unexpected ways. I present my findings from a demographic exploration of eponymization in rhetoric and composition; I conclude with a distant-rhetorical analysis of eponymous adjective use, taking as a representative example uses of the word "Bakhtinian," offering some hypotheses on the processes of eponymization. 


\section{Why Eponymous Adjectives?}

Before I consider eponymous homogeneity in more detail, I wish to explain first how and why I came to consider eponymous adjectives. Allusion is the secret handshake to citation's high-five. It acknowledges disciplinary or literary knowledge shared between reader and writer, without calling unnecessary attention to itself. And in some cases, authors do less citation and more allusion as they become more established. ${ }^{28}$ In one of his last publications, The Archaeology of Knowledge, Foucault cited formally just four times; his first major publication, Mental Illness and Psychology, had more citations in the first two pages than The Archaeology of Knowledge had throughout. Yet that later work was full of reference, rapid-fire roll calls that ask authors' names to stand in for their entire body of work. Here, for instance, is a representative three-sentence excerpt from Archaeology:

$[T]$ o what extent can one affirm that it is the same organicist theme that emerges in the so very different discourses and vocabularies of Buffon, Jussieu, and Cuvier? And, inversely, can one say that the word 'organization' has the same meaning in the work of Daubenton, Blumenbach, and Geoffroy Saint-Hilaire? (p. 143)

Not one of these references identifies a particular publication, let alone a line or page number. I wondered, thus, when I realized that the most-cited authors cited less, whether they might be doing more informal reference. But at first, I could not figure out how to get at this kind of allusion with distant reading, without much time-consuming manual coding: Only a complex algorithm, one beyond my coding skills, could distinguish between an author's named paired with a formal citation and an author's name that stands

\footnotetext{
${ }^{28}$ An analytic category that was at one point central to this chapter's analysis; I found some evidence to suggest that authors who are neither newcomers nor "most-cited" authors use more eponymous adjectives than other groups. I have removed the idea from this chapter for brevity and a stronger through-line, but I expect I will return to the question in some future work.
} 
alone, a la Foucault's roll call above. I was not sure how to distant read this kind of Foucauldian thing. But then, musing on that word—Foucauldian — brought me to one way of getting at informal reference in a distant reading sense: I could search for adjectival forms of authors' names, which are used almost exclusively in that "secret handshake" sense, almost never paired with a formal citation. They are formed in predictable ways, with “-an,” “-ian,” and "-esque” endings.

Hence, I began to compile a list of eponymous adjectives that might show up in my corpus. The Wikipedia "List of eponymous adjectives in English" was at the time of my data analysis 401 names strong; first, I distilled from that list any that might plausibly show up in English scholarship, and checked for each in the corpus. I then went through each of Mueller's "most-cited" names and checked my corpus for as many adjectival variations on a name as seemed plausible. For instance, I looked for "hooksian" and "hooksesque," "Villanuevan and "Villanuevian"-but found quickly that some names did not show up at all (for instance, "Geeian," which in its vocalic excess, stretches the bounds of pronounceability ${ }^{29}$ ). From these two lists, and with a few others distilled from this, and with a few more literature and rhetoric and composition-specific additions (e.g. Rortyan, Toulminian), I developed a list of 27 eponymous adjectives that appear in my corpus. $^{30}$

The practice of using eponymous adjectives turned out to be relatively common523 of my 1,327 articles (just shy of $40 \%$ ) used at least one eponymous adjective. There

\footnotetext{
${ }^{29}$ I recognize that "pronounceability" is itself a characteristic that draws demographic boundaries around names; names that accept "-ian" or "-esque" endings are more likely to be of Latinate or Germanic origins, respecitvely.

${ }^{30}$ Aristotelian, Bakhtinian, Burkean, Butlerian, Chomsk[i][y]an, Cixousian, Derridean, Deweyian, Foucaul[d][t]ian, Freire[i]an, Freudian, Hobbesian, Homeric, Jungian, Marxist, Murrayesque, Northian, Ongian, Platonic, Rortyan, Saussurian, Shakespearean, Shorian, Socratic, Toulminian, Vygotsk[i][y]an, Yanceyan
} 
does seem to be a shift away from eponymizing, if a slow and unsteady shift, with several significant outliers (see Figure 3.6):

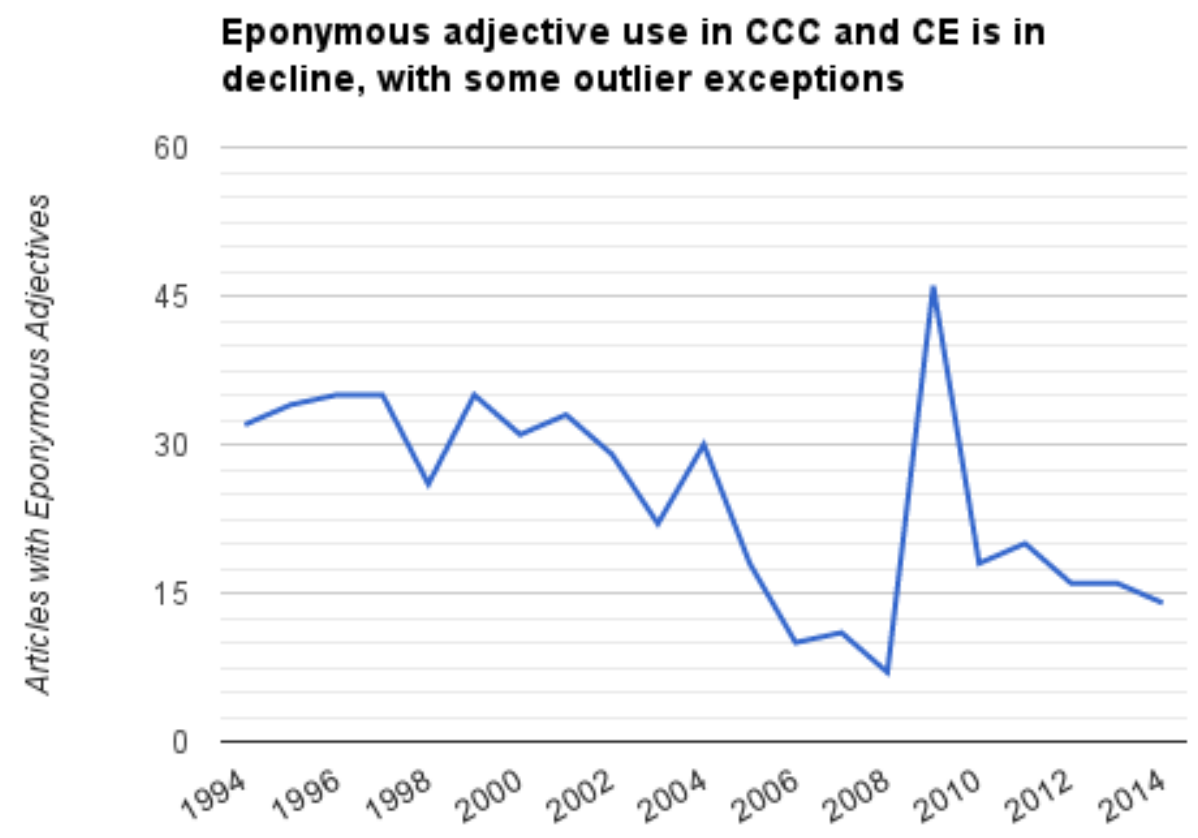

Figure 3.6: In the twenty years between 1994 and 2014, in $C C C$ and $C E$, the number of articles using at least one eponymous adjective declined appreciably_if unsteadily.

Nevertheless, eponymous adjective use is a practice that remains prominent, and one with which authors expressed (somewhat disdainful) familiarity in our interviews. Thus, I am calling this practice the "slow burn" of paracitational homogeneity: Even as the practice declines, even as authors seem to view it as somewhat contempt-worthy, it continues to exert force on our discipline. We continue to reproduce, in our eponymous adjectives, very homogenized assumptions about what knowledge can be assumed to be shared, about what scholars are worth eponymizing - and thus, with every eponymous adjective, we continue to compound the effects of the underrepresentation of groups of alreadymarginalized scholars. 


\section{The Matilda Effect}

Though few would be surprised to hear that authors' race and gender affect the ways they are cited, more surprising is the power and universality of what Margaret W. Rossiter (1993) called the "Matilda effect," the systematic underrecognition of academic women. In a citation corpus analysis of two major communication journals, Silvia Knobloch-Westerwick and Carroll J. Glynn (2013) found that male-authored papers were much more likely to get cited: "Publications with female lead authors were cited 12.77 times on average $(\mathrm{SD}=20.57)$, whereas publications with male lead authors ${ }^{31}$ were cited 17.73 times on average ( $\mathrm{SD}=35.34)$ )" (p. 13). Even when female lead authored publications are picked up widely, however, it is common for media reports of those publications to reorder the authors' names. Justin Wolfers (2015), in a New York Times:

The Upshot post titled "Even Famous Female Economists Get No Respect," described three instances in which media reports of female first-authored economics papers had mistakenly swapped the female economists with their male coauthors, also oddly stripping the women of impressive academic credentials. University of Rochester anthropologist Signithia Fordham (2014) described a similar experience: Though Fordham was first author of her famous and widely cited research about black high school students' academic success (Fordham and Ogbu, 1986), when Barack Obama discussed "acting white," an MSNBC report "included an image of Ogbu as the sole source of the theory" (Fordham, 2014). A 2005 Duke University conference themed

\footnotetext{
${ }^{31}$ I reproduce this study's untroubled gender binary here and throughout this chapter with the knowledge that a complete study would ask authors to self-identify and provide non-binary gender categories. Knobloch-Westerwick and Glynn (2013) had three coders identify each name; when the coders were unsure, they checked authors' personal websites. For my own gender typing in Table 2, Academic slowness to recognize non-binary gender is a related, significant topic just
} 
around the paper committed a similar error, mistakenly inverting the paper's authorship, calling it "Ogbu and Fordham's Hypothesis."

Site-specific and interdisciplinary analyses have no difficulty documenting the "Matilda effect" in formal academic citation practices, ${ }^{33}$ yet I suspect that the effect is still bigger in the informal practices of reference: That is, when academics are working from memory and a felt sense of shared knowledge, their reproduction of biases, gender and otherwise, is likely larger than when they cite formally.

\section{Who Gets Eponymized?}

Who, then, gets eponymized, and why? Questions of gender, race, geographical location, and discipline loomed large both in my own analysis and in my discussions of eponymous adjectives with journal editors Kelly Ritter and Jonathan Alexander. When I began my collection, I struggled at first to come up with female eponymous adjectives: Just 15 of the 401 eponyms $(3.74 \%)^{34}$ on the Wikipedia list have female origins. They come mostly from queens and religious figures, derived often from first rather than last names, with just three women remembered for their thought or writing (Noethe, Rand, Sappho). Given the fact that just $21.9 \%$ of JSTOR's collection, including papers

\footnotetext{
${ }^{32}$ For these examples, I am indebted to a series of February 2016 exchanges on the Association of Internet Researchers listserv (Air-L): In the thread titled "Please Cite Women Academics," Gabriella Richard of Penn State pointed me toward Fordham's story; in the thread titled "Gender," Lisa Tagliaferri of the City University of New York pointed me toward Davona Savonick and Cathy Davidson's ongoing Google Doc documenting academic studies of gender bias in academe; in the thread titled "additional pieces of bias against women in journalism," Cecilia Aragorn of University of Washington pointed me toward the Eigenfactor analysis of gender distribution in JSTOR.

${ }^{33}$ See King, Correll, Jacquet, and Bergstrom (2015) for a JSTOR-wide analysis of gendered self-citation; Lutz (1990) for a gendered citation analysis of four anthropology journals; Maliniak, Powers, and Walter (2013) for an international relations citation analysis; and Knobloch-Westerwick, Glynn, and Huge (2013) for an analysis of evaluations of science conference abstracts.

34 For the curious: Chlumskyan, Elizabethan, Fatimid, Imeldific, Junoesque, Marian, Marlenesque, maudlin, Noetherian, Polyannaish, Randian, Sapphic, terpsichorean, Victorian, and Vestal.
} 
published from 1665-2011, have female authors, and given Rossiter's (1993) "Matilda effect," this disproportionality makes some sense.

One might expect more equitable gender and racial representation in rhetoric and composition scholarship, given the increasing diffusion and diversification of our citation practices more broadly. We are citing more people, less often in the "core" of the discipline — to the extent that some have challenged the notion of a core: Mueller's (2012) citation analysis suggested that our field is becoming less focused on work by a small handful of superstar authors and more open to work by less-established authors. $\mathrm{He}$ described a growing "long tail" of unique names cited in $C C C$, from about 1,300 names in 1987-91 to over 3,000 names in 2007-11 (p. 211-13). From 2007-11, he explained, "The most frequently cited figure—Kathleen Blake Yancey—was invoked in scholarly articles roughly half as often (26 references) as Linda Flower was in the comparable period of time twenty years earlier (56 references)" (p. 215). This diffusion makes sense, given the changing shape of our field. Journals have proliferated: the Council of Writing Program Administrators currently names 42 journals in rhetoric and composition. So, too, have doctoral programs: in the 10 years since Rhetoric Review's 2007 survey (Brown, et al., 2007), the Doctoral Consortium of Programs in Rhetoric and Composition has grown by 10 , from 68 to 78 . "Burke's parlor is nowadays full and teeming, more crowded than ever before," Mueller explained. "Even while the head of the distribution stands tall—an indication of the recurrence of fairly regular, recognizable names (e.g., Linda Flower, Patricia Bizzell, Peter Elbow, etc.) - the long tail tests the limits of comprehension and memory" (p. 214-15). 
Why, Then, Are Our Eponymous Adjectives So Homogeneous?

Yet Seth Long's (2014) analysis of citations in abstracts from CCC, Rhetoric Society Quarterly, and Rhetoric Review from 2000 - 2011 found that rhetoric and composition's major journals have unequal gender citational distribution: In $C C C$, Rhetoric Review, and Rhetoric Society Quarterly, 71\% of cited authors were male. The disparity is even more dramatic in our eponymous adjective use, which is dramatically homogeneous (see Table 1). 


\begin{tabular}{|c|c|c|c|c|c|c|}
\hline EPONYM & FIELD & GENDER & LIVING? & COUNTRY & $\begin{array}{c}\text { \# of CCC } \\
\text { articles }\end{array}$ & $\begin{array}{l}\text { \# of CE } \\
\text { articles }\end{array}$ \\
\hline Aristotelian & rhetoric & $\mathrm{M}$ & dead & Ancient Greece & 37 & 32 \\
\hline Bakhtinian & literature & $\mathrm{M}$ & dead & Russia & 13 & 18 \\
\hline Burkean & rhetoric & $\bar{M}$ & dead & United States & 33 & 18 \\
\hline Butlerian & $\begin{array}{l}\text { gender } \\
\text { theory }\end{array}$ & $\mathrm{F}$ & alive & United States & 1 & \\
\hline Chomsk[i][y]an & linguistics & M & alive & United States & 3 & 2 \\
\hline Cixousian & $\begin{array}{l}\text { literature/ } \\
\text { rhetoric }\end{array}$ & $\mathrm{F}$ & alive & Algeria/France & 1 & \\
\hline Derridean & philosophy & $\mathrm{M}$ & dead & Algeria/France & 1 & 12 \\
\hline Deweyian & philosophy & $\mathrm{M}$ & dead & United States & 1 & 2 \\
\hline Foucaul[d][t]ian & philosophy & $M$ & dead & France & 16 & 26 \\
\hline Freire[i]an & education & $M$ & dead & Brazil & 17 & 17 \\
\hline Freudian & psychology & $M$ & dead & Austria & 7 & 20 \\
\hline Hobbesian & philosophy & $\mathrm{M}$ & dead & England & 1 & \\
\hline Homeric & literature & $M$ & dead & Ancient Greece & 2 & 5 \\
\hline Jungian & psychology & $\mathrm{M}$ & dead & Switzerland & 1 & 5 \\
\hline Marxist & philosophy & $M$ & dead & Germany & 41 & 73 \\
\hline Murrayesque & rhet/comp & $M$ & dead & United States & 1 & \\
\hline Northian & rhet/comp & $\mathrm{M}$ & alive & United States & 1 & \\
\hline Ongian & literature & $\bar{M}$ & dead & United States & 2 & 1 \\
\hline Platonic & philosophy & $\mathrm{M}$ & dead & Ancient Greece & 22 & 28 \\
\hline Rortyan & philosophy & $M$ & dead & United States & 3 & 1 \\
\hline Saussurian & $\begin{array}{l}\text { linguistics/ } \\
\text { semiotics }\end{array}$ & M & dead & Switzerland & & 3 \\
\hline Shakespearean & literature & $\mathrm{M}$ & dead & England & 3 & 23 \\
\hline Shorian & rhet/comp & $M$ & alive & United States & & 1 \\
\hline Socratic & rhetoric & $M$ & dead & Ancient Greece & 5 & 13 \\
\hline Toulminian & philosophy & $M$ & dead & England & 1 & 2 \\
\hline Vygotsk[i][y]an & education & $\mathrm{M}$ & dead & Russia & 2 & 5 \\
\hline Yanceyan & rhet/comp & $F$ & alive & United States & 1 & \\
\hline
\end{tabular}

Table 3.1: Eponymous adjectives in $C C C$ and $C E$. Mostly male, mostly dead, mostly Western, mostly outside of the discipline. 
Most of our eponymous adjectives are male: Just 3 of the 27 adjectives (11.1\%) are female. Most of the authors are from outside of rhetoric and composition proper: Just 4 of 27 (14.8\%) are soundly located within our field — adding classical rhetoricians and Helene Cixous brings that number up by 4 (to 8 of 27 , or $29 \%$ ), still clearly the minority of eponymizations. Perhaps more predictably, most of the authors represented are dead: Just 6 of $27(22.2 \%)$ are alive - a finding that makes some sense, given the fact that eponymization tends to follow a "completed" shared public understanding of an author's body of work. Becoming eponymized is a high academic honor; it suggests that one's work is so well known that readers will not need either an author's first name nor a pointer to a particular text to understand the reference. The dead, thus, are understandably more often eponymized.

Somewhat more surprising, though, is the finding that nearly all of the authors represented are white and Western: None of the authors represented are nonwhite—and just 3 are from arguably non-Western countries. To emphasize how dramatic this finding is, let me belabor briefly: That means no eponymized black scholars-no bell hooks, no Geneva Smitherman, no Jacqueline Jones Royster, no Keith Gilyard, no Adam Banks. No eponymized Asian scholars-no Min-Zhan Lu, no Maxine Hong Kingston, no A. Suresh Canagarajah. And Paulo Freire is the only scholar on the list who might count as Latino or Hispanic (neither of which, of course, are particularly well-defined categories)—no Victor Villanueva. Nonwhite scholarship is, of course, represented in formal citation and in other kinds of informal reference — but eponymous adjectives offer a quick read on disciplinary centrality, the sort of collective unconscious of our field. And the absence of nonwhite scholarship in this list is telling. 
When it comes to country and area of origin, the list is hardly any more diverse. Ten of the 27 (37\%) are American; 14 of 27 (51.9\%) are European; all 27 are Western, according to the cultural, Judeo-Christian definition of the term. ${ }^{35}$ The Western bias and high theory bias of the list are, I believe, mutually informing: The pillars of both stand in Ancient Greece. We reference Aristotle and Homer and Plato and Socrates with the knowledge that anyone educated in language or literature in a Western university must have been exposed to all four. Even famous ancient philosophers from other traditions (e.g. Laozi, Sun Tzu, Siddhartha Gautma, Averroes) are much less often canonized and thus less likely to be referenced sans gloss.

This finding offers evidence for bell hooks's (1992) suggestion that white scholarship sometimes gets unproblematically considered "the only relevant discourse." Many translingual composition and rhetoric scholars have considered the impact of Western cultural imperialism on the nature of academic publishing. Anne-Marie Pedersen (2010), for example, described public Jordanian university tenure and promotion policies that

reward publications in Western, English-language journals more than they reward publications in Arab and Arabic-languages journals. [...] This devaluing exacerbates an already difficult situation for non-Western, non-English journals: because scholars who publish in prestigious, indexed journals often devalue and, therefore, ignore non-Western, non-English journals in their research, these publications are rarely cited. And because they are rarely cited, they are rarely indexed, as indexes generally include only the journals that are regularly cited in other articles. (p. 294-95)

\footnotetext{
35 Definitions of "Western" are rather hotly contested-Samuel P. Huntington's (1996) "Clash of Civilizations" proposed nine major civilizations, arguing that Russia (from which 2 of 27 eponyms, or $7.4 \%$, are drawn) and all previously Soviet nations are distinct enough from European imperialism that they ought to be considered separately; he was unsure whether to count Latin America as part of the West. The Judeo-Christian definition, however, considers Western according to the success of Western culture's greatest major export: Christianity. According to this defintion, Russia and Brazil are soundly Western.
} 
Even in countries without publishing guidelines like these from Jordan that explicitly value English work higher, publishing in a language other than English today can have serious academic consequences. As Ken Hyland (2009) pointed out, “[A]cademics all over the world are ever less likely to publish in their own languages and to find their English language publications cited more often" (p. 18). And A. Suresh Canagarajah (2014) identified how such citation "affects knowledge construction," when one group of authors writing about pragmatics of local teaching felt compelled to scrub their article of references to local journals (which were often non-English and lower-tier): "The authors of the collaborative article cited only one article from their own nation. [...] Eventually, it appeared as if the authors were simply using local data to conduct a disciplinary conversation that is denuded of social context. The citations built a discoursal world that compels us to read the local data in terms of an 'unplaced' disciplinary discourse" (p. 10). Thus, there is a way that citational homogeneity can come to seem natural, even inevitable, and can in the process limit the potential richness of our disciplinary discourse.

In pointing to all of this homogeneity, I do not, again, mean to finger-wag; a similar investigation of other humanities disciplines would likely find equivalent levels of homogeneous eponymous adjectives. Rhetoric and composition is far from the only discipline that stands on the shoulders of Aristotle and Dewey and Derrida — that assumes shared knowledge of more dead scholars than live ones, more white scholars than black, more Westerners than non-Westerners. But eponymizing is a practice, one among many, that reproduces and compounds the effects of the underrepresentation of minority discourses. Thus, in the interest of digging into "scenic unconscious practices" that 
present racism as "business as usual," as Catherine Prendergrast (1998) put it, we do well to identify the dramatic racial and gender disparities in eponymization.

And here, another impasse, my second aporia: Recognizing that eponymous adjective use reproduces and compounds underrepresentation does not change the fact that scholars who make up our shared knowledge base come from the long academic past of demographic boundaries — where official knowledge-making was whiter, more male, more Western than we believe it to be today and hope it will be tomorrow. How, then, to move forward? For some insight, I turn (like any good rhetorician) to rhetorical analysis, aided by my distant-reading methods:

\section{Rhetorics of Eponymization: How We Use Eponymous Adjectives}

To develop a deeper understanding of how we use eponymous adjectives, I decided $^{36}$ to investigate the rhetorical uses of one particular eponym: I picked "Bakhtinian" both for its apparent typicality (Bakhtin is a dead, Western, high-theory male) and its atypicality (Bakhtin was, of course, "rediscovered" many years after he was writing; his work is incorporated into rhetoric and composition scholarship in translation; our scholarship has appropriated from his work at least a half-dozen term that have become popular analytics_ —"heteroglossia," "polyphony," "carnival," "centripetal/centrifugal forces," "chronotope," and of course "appropriated" itself). Of the eponymous adjectives I found, it was the 7th most common, with 31 uses in $C C C$ and $C E$

\footnotetext{
${ }^{36}$ To push back against the "phony story" of research, let me elaborate a little on "decided": In response to the first full draft of this chapter, both Drs. Bronwyn Williams and Carolyn R. Miller expressed concerns about the ways I was ascribing too much agency for eponymous bias to individual authors and not considering either the ways other fields are similar or how rhetoric and composition is unique. Dr. Williams recommended I take one author as a case study; we spent time in his office discussing some of the ways people have picked up "Bakhtinian," so I went back to my corpus with the goal of understanding the rhetorical uses of that eponym.
} 
combined, from $1994-2014$. I generated a list of all the articles that used the term and extracted the relevant sentences. The sentences divided rather neatly into five categories (see Figure 3.7): Articles that described another author's use of Bakhtin (13 uses, ${ }^{37}$ or 41.9\%); articles that simply cited another work with "Bakhtinian" in the title (5 uses, $16.1 \%)^{\prime}$ articles that used the term to gloss all of Bakhtin's theories as a whole (5 uses, $16.1 \%)$ ' articles that used "Bakhtinian" to define a terministic screen for the article more generally (4 uses, or 12.9\%); and articles that used "Bakhtinian" as a central analytic category (4 uses, or 12.9\%).

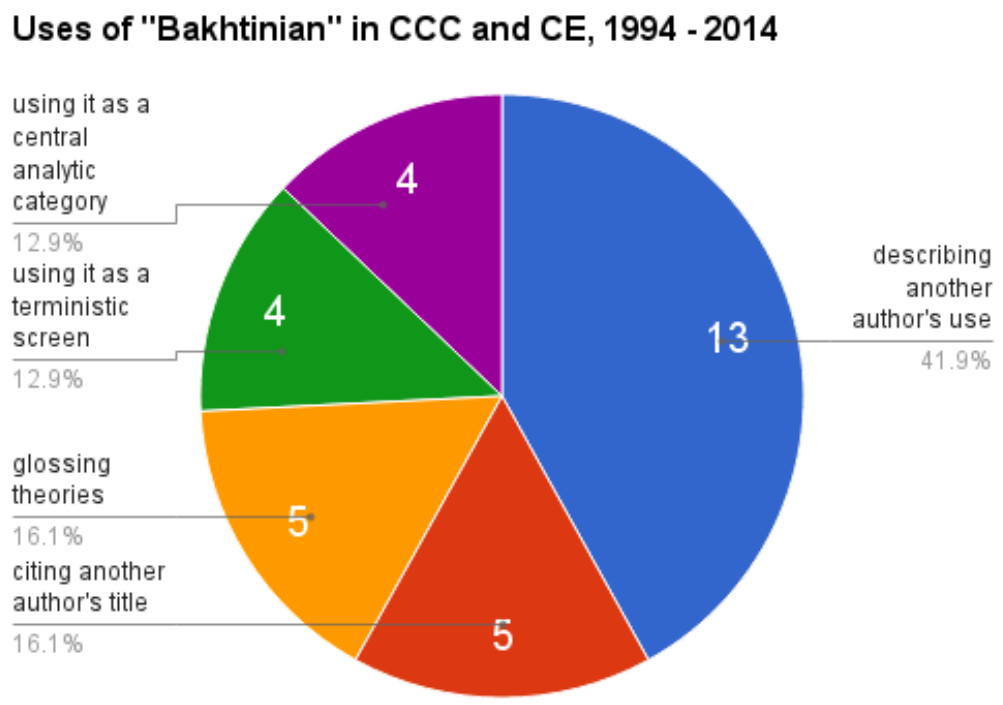

Figure 3.7: Number of articles that used the eponymous adjective "Bakhtinian" in $C C C$ and $C E, 1994-2014$, divided into categories of use.

\footnotetext{
${ }^{37}$ I counted multiple instances of the term as a single "use," as long as each use within an article fit within the same category.
} 
The vast majority of articles, that is, used "Bakhtinian" either as a means of nodding to the fact that an author cited in their own text relied heavily on theories from Bakhtin. Here is Asao Inoue (2013), for instance: "Vivette Milson-Whyte's policy chapter offers a Bakhtinian explication of the way Gina Valdes's poem, 'English Con Salsa,' is a published instance of code-meshing suitable for studying with writing students"; similarly, Jude Chudi Okpala (2010): "Gilyard accepts that solution, and does so with salient analysis of African American vernacular tradition as a classic application of Bakhtinian dialogism and as a rich, liberatory discourse." In a lot of these cases, I believe "Bakhtinian" simply serves as a way of sidestepping the clunkier formal methods of referencing a work that is referenced in another work-MLA's awkward indirect citation "qtd. in" construction, e.g. (Bakhtin, qtd. in Milson-Whyte 32). The next-most-frequent uses of "Bakhtinian" are as a word in the title of a cited work (three authors referenced Kay Halasek's A Pedagogy of Possibility: Bakhtinian Perspectives on Composition Studies) and as a means of glossing "Bakhtinian theories" broadly in the course of a longer discussion of Bakhtin. Michael Bernard-Donals (1994), for instance, said, "If one simply extrapolates Bakhtin's work on individual subject relations and cognition to his works on broader social relations constructed by and with language, one can conceivably build a unified and consistent Bakhtinian language theory." A few authors similarly used "Bakhtinian" to set up what Burke called a "terministic screen" for the essay, borrowing a Bakhtinian concept such as "Bakhtinian dialogism" (Juzwik, 2004) or "Bakhtinian carnival" (Rouzie, 2001). Finally, four of the essays used "a Bakhtinian approach" (Farmer, 1998) to research, taking a term or concept or category from Bakhtin and 
structuring the entirety of a study or other analytic project around it, such as Jeffrey $\mathrm{T}$. Nealon's (1997) riffing on the nature of "Bakhtinian selfhood."

None of these uses are deliberately exclusionary; though many of the eponymizings could be replaced with a more traditional, formal citation of Bakhtin, none would make sense if replaced with a similar eponymizing of a less-well-known scholar. The purpose of the term, in each case, is as a convenient shorthand for a broad swathe of ideas that the author reasonably assumes the reader will recognize. In the case of Bakhtin, the rhetorical velocity (Ridolfo and DeVoss, 2009) of his ideas has contributed significantly to his eponymizing; the fact that most rhetoric and composition scholars can name a half dozen or more concepts that we have, as a field, adopted from his work, makes his name an especially ripe eponym.

Why Murray, North, Shor, and Yancey?

In some ways, the tiny core of eponymized authors makes sense: Perhaps in part because we are citing more widely, we cannot assume shared knowledge of many of the authors in rhetoric and composition. For an author to become eponymized in $C C C$ or $C E$, he or she must be so well recognized that all the people reviewing the eponymizing article must agree that it is reasonable to assume readers will recognize the name. Jonathan Alexander further proposed that eponymization might reflect the extent to which an author's work has significant play outside a given discipline, its exportability: You get eponymized when your work is kind of architectonic to multiple areas or multiple venues of exploration. We're a field. Foucault is not a field. Foucault influenced multiple fields of inquiry and probably created some along the way. 
[...] I don't know that we are at the point where members of our field have the sort of reach across disciplines that would validate that kind of eponymization. Foucault, who represents 42 of the 523 (8\%) eponymous adjectives in my corpus, does seem to share a degree of exportability with many of the other most-eponymized authors, from Aristotle to Bakhtin to Burke to Freire to Freud, each of whom could be easily claimed by two or three or more disciplines. The notion that rhetoric and compositionists are not exporters is a key topic of Karen Kopelson's (2008) CCC report of a disciplinary survey, in which she described our field's "one-way interdisciplinarity": "Though we have long foraged about in other bodies of knowledge — and, yes, to some innovative and crucial ends — we are still primarily importers only, consumers, an 'interdisciplinary' field, if it can be said that we are one, with little to no interdisciplinary influence" (Kopelson, 2008, p. 768). Perhaps that notion of exportability might explain why two of the four rhetoric and composition scholars who have been eponymized made the list: Donald Murray was a Pulitzer Prize-winning journalist in addition to his academic rhetoric and composition work. Ira Shor, in his close work on critical pedagogy with Paulo Freire (e.g. A Pedagogy for Liberation, 1987), has had a broader influence on education outside of the first-year composition classroom than perhaps any other rhetoric and composition scholar. Outside of Mike Rose (who I can only imagine escaped eponymization because "Rosean" and "Rosesque" seem unnatural, and "Rosey" and "Roseish" seem cutesy), they are likely about as close to "household names" as rhetoric and composition gets.

But these two eponyms, each of which appears just once in my corpus, actually also demonstrate another, less flattering side of eponymization: "Murrayesque" occurs in 
Wendy Bishop's (1999) "Places to Stand," where she reports a reviewer who "once refused an essay by Donald Murray on the grounds that it was too 'Murrayesque' to be included in that journal (that is, contrary—as he saw it— to the journal WPA's professional mission)" (p. 13). The single appearance of "Shorian" serves a remarkably similar purpose, reporting on another scholar's critique of Shor: Julie Lindquist's (2004) “Classroom Affects, Classroom Affectations" describes Gwen Gorzelsky's critique of "traditional Shorian pedagogy" that "doesn't fully recognize the critical potential of the affective dimesnion" (p. 191). None of the 31 uses of "Bakhtinian" I investigated had this kind of sneering tone; why, when we eponymize these rhetoric and composition scholars, do we do so dismissively? "Bakhtinian" is instrumental in a lot of cases —using the eponym as a handle for a borrowed concept from Bakhtin's vast catalogue. Perhaps there is something more dismissive in the project of eponymizing someone alive (Shor) or field-foundational (Murray).

The other two eponymized rhetoric and composition scholars, Stephen North and Kathleen Yancey, do not boast the same kind of out-of-field exportability-yet they are representative of another potential explanation Jonathan Alexander offered for eponymization, the way by which individual articles can "achieve a sort of shorthand status." He pointed out that Yancey's (2004) CCCC chair's address, which was published in the December CCC, "Made Not Only in Words: Composition in a New Key," has become a shorthand for multimodality. When people want to talk about multimodality, Alexander said, they seem almost obligated to reference Yancey's address:

Those articles can sort of stand in for a whole movement of the field, a whole sensibility emerging in the field. So it makes sense that her article would come to be in some ways overly cited, I think, to the detriment of other work on 
multimodality, multimedia that's probably just as interesting, that needs to be heard.

That address has had wide reach within the field; in the dozen years since its publication, Google Scholar reports that it has been cited 325 times. The single use of "Yanceyan" in my corpus is not, in fact, citing that CCCC address; rather, it appears in Chris Gallagher's (2014) review essay, "All Writing Assessment is Local," which considered how Asao Inoue and Mya Poe (2012) "address a Yanceyan self-created exigence: the need to elucidate how race functions in writing assessment in order to make our local assessments fairer" (p. 493). Yet Gallagher's use of "Yanceyan" nevertheless reflects a sense that readers will recognize in the term a particular style of approach, a particular attitude toward scholarship.

Kelly Ritter had a very similar explanation of why Stephen North might have been eponymized - he has become field-famous for two widely cited pieces:

Everybody knows what North did. They know two things about him. They know that he wrote Making of Knowledge in Composition [1987] and "The Idea of a Writing Center" [1984]. There's nobody in rhet/comp who doesn't know both of those things. Unless they're in a coma.

Making of Knowledge has been cited 837 times since 1987, and "The Idea of a Writing Center" has been cited 584 times since 1984. The idea of a piece achieving "shorthand status" is sort of a more localized, particularized version of exporting — an individual idea gets exported from an article or book, and the idea becomes represented metonymically by the author's name. Coincidentally, the single use of "Northian" in my corpus comes from Douglas Hesse's (2010) “The Place of Creative Writing in Composition Studies,” which describes Kelly Ritter and Stephanie Vanderslice's discussion of "the lore of the 
workshop," in "the Northian sense"-referencing, of course, North's notion of "lore" from Making (p. 36).

My ultimate point in discussing the processes of eponymization for these four authors is not to suggest that they are exceptional, either among rhetoric and compositionists or among scholars who get eponymized. Rather, I hope to suggest that these are the processes by which power structures become reinforced: Quietly, apparently self-evidently, without good reason to criticize any individual instance of the reinforcement. That is, surely few who use the terms "Marxist" or the term "Foucauldian" do so with the aim of inculcating white values, or of limiting the reach of rhetoric and composition scholarship, or of barring women from the canon. In fact, these words show up in plenty of scholarship that aims at precisely the opposite. Yet we are all, in our word choices, and in our naming practices, complicit in keeping some of these power structures intact.

Eponymization is not, as I discuss above, necessarily done in a positive light. Yet it reflects our assumptions about what knowlege can and should be shared. I suggest, thus, that this analysis of our eponymization practices points to the ways our discipline is built upon much whiter, male power structures than we tend to admit. My call here is not necessarily “eponymize more women!" or “eponymize more black people!"- though I certainly would not mind if we talked about Selfeian understandings of technology or Smithermanesque approaches to language diversity. Changing naming practices will not, ipso facto, change power structures. But eponymization is one significant piece of a wide and long-standing puzzle, a piece that reflects deeper structures of power and status. When we are writing about work that "everyone knows," we tend to refer to the author 
less formally. Because of the ingrained power structures behind the "Matilda effect," general knowledge has a male bias. Because of Western cultural imperialism, general knowledge has a Western bias. Because rhetoric and composition can assume little shared disciplinary knowledge beyond literature, general knowledge in $C C C$ and $C E$ has a high theory bias.

\section{Where Do These Aporias Leave Us?}

Through a distant reading into the disciplinary narratives about our citation practices, I have identified two aporias, two places where our narratives butt heads either with themselves or with our practices:

1. We circulate two competing disciplinary narratives about newcomers: One that suggests newcomers cite too much (and my data suggests they cite a lot indeed), and another that suggests they do not cite enough (seems counter to some of my data, though newcomers are far less likely to have an extremely high-citation-count article).

2. Against the narrative of increasing disciplinary openness and explicit attention to race and gender, I have investigated the paracitational practice of eponymous adjective use, revealing a slow burn of academic hegemony that, through our assumptions about shared knowledge, reproduces biases and compounds the effects of underrepresentation.

In both cases - and as throughout this dissertation-I aim to identify citational practices that have so far remained tacit. From those practices, I seek deeper understandings of values, of histories, and of the kinds of pressures that quiety exert themselves on authors at the moment of citation. 
I recognize that citations reflect far more than disciplinary orientations. When we write, we juggle countless local and global, personal and political concerns. Of these, "citing more women" and "citing more scholars of color"—or "not citing like a newcomer" or "making sure I cite enough to look knowledgable"-may be goals indeed. But they are goals among many, and they are goals that may get subsumed under more important local and content concerns, such as, "Does this quote support my point?" and “Am I connecting to enough recent scholarship?” In the case of eponymous adjectives, my investigation of uses of "Bakhtinian" suggests that the primary driving factor behind such practices is expediency—gesturing at another author's use of a scholar's work without needing to engage in the clunky practice of secondary citation. Yet distant reading's greatest value for my study here is that it can assess, to some extent, the aggregate results of this juggling: How successful are we, that is, at responding to the values and concern we say most interest us? Distant reading provides a broad judgment of our citational relative balance.

\section{A Pragmatic Turn, Toward the Next Chapter}

As throughout this dissertation, I have in this chapter been working to investigate tacit practices, teasing out the undertheorized, even unconscious habits and expectations that drive academic work. Yet this chapter has been a bit of a departure from the explicit aims of the rest of this dissertation: I have focused in this chapter not on web practices, not on hypertext, not even on transitions from print to hypertext. I have turned instead to traditional print academic citation practices, with the aim of understanding some of the more basic notions that underlie academic citations, some of the values that authors in rhetoric and composition engage when they face the stack of library books and folders 
full of PDFs that hide behind every published article. Traditional academic citations, this investigation has found, serve a wide variety of purposes, from demonstrating "insiderness" to glossing big broad areas of disciplinary conversation, to connecting otherwise disparate communities that make up the citational base of a piece of work.

Now, as I begin to turn toward the next chapter, which returns to hypertext and attempts to draw some connections between the popular press practices of Chapter II and the traditional academic practices of Chapter III (through an investigation of academic web writing), I find myself looking for some pragmatic takeaways. A few such takeaways:

- Learning to cite within a community of practice is a slow, iterative process;

- Citation practices are not stable within a community of practice or, in most cases, even within an individual author's work — and thus, as a corollary:

- As people become more comfortable in a field, they cite differently from how they cited early on;

- Citation practices reflect disciplinary anxieties, and those anxieties shift as authors become more established within academia;

- Assuming shared knowledge in one's citation practices can reproduce biases that are inherent to academic practice;

- Less assumption of shared knowledge improves the rhetorical velocity of a text, in that it becomes more easily picked up by people from different stations 
Thus, my ideal "academic web publication for the public"-for which I develop a proposal in the conclusion to this dissertation —-would not assume shared knowledge of texts. It would ban the eponymous adjective in favor of some kind of citation that gives newcomers somewhere to hang a hat, somewhere to turn if they cannot rely on shared knowledge. This publication would aim to be as explicit about citation practices as possible, with the goal of easing newer authors through the anxieties of citing in a new space. It would offer a set of rhetorical, flexible guidelines - guidelines that reflect awareness of the complexity of citation practices, avoiding broad admonitions ("cite more women," "cite more people of color") with the understanding that local concerns often outweigh ideal concerns. Yet these guidelines would also give authors a sense of the importance of citation practices in sketching out the intended and potential audiences of a piece of writing.

What, then, is "phony" about our citational stories? Though citations often are clustered in the opening few pages of an article, stuck into the Swales-move introduction or woven together in the first few pages of literature review, citations rarely precede our work like this in such fully-formed versions of the academic lineage of our work. There are much more complex pressures being exerted on authors as they write, and though those pressures can result in a neat-looking syllogistic kind of citation story ("first, I read this; it spoke to that; and that, of course, brought me to reading this — and ergo, my 'gap"'), that story does not reflect the actual values embedded in citation or the kinds of anxieties that authors experience as they decide when, where, and how to cite. By distant reading our citation practices, we can triangulate disciplinary narratives about citations, 
and find in that triangulation a less-phony story, or at least another story to read against the phony story. Perhaps "less-phony" is our best hope. 


\section{CHAPTER IV}

\section{THE NEW ALLEGIANCES OF ONLINE WRITING: WEBTEXT CITATION PRACTICES AND WHY WE NEED A DIFFERENT KIND OF PUBLICATION}

At the outset of this dissertation, I explained how I came to approach this project with two major concerns: (1) how academics might reach wider public audiences, and (2) how and why people cite the way they do. Both of the previous two chapters primarily addressed aspects of my question about citation practices - Chapter 1 focused on popular press hypertext practices, forwarding a "rhetoric of hypertext"; Chapter 2 focused on academic print citation practices, arguing that our values and practices often do not align. While my previous chapter had implications for reaching wider public audiences, until now, I have only addressed the question of public sharing obliquely. In this chapter, I return to the question that I borrowed from Harlot at the outset of the dissertation: "If this stuff matters" - that is, if popular presentations of academic work in the humanities matter, as I think they do_- “why isn't it being shared?" This chapter focuses intently on that public question through analysis of yet another kind of citation, academic hyperlink webtext citations; I use interviews with authors and editors of academic webtexts in rhetoric and composition to ferret out the underlying assumptions and values that drive 
web writing. Ultimately, I develop a list of four key allegiances ${ }^{38}$, shared among the majority of my participants: kairotic exigencies, availability, attention, and sharing.

Discussing hyperlink practices is a telling way of uncovering allegiances that might otherwise remain tacit, because (1) they are practices that necessarily differ from traditional print writing practices, and (2) they are nevertheless relatively unexplicit. The authors and editors I interviewed had not, as a universal rule, been given any guidelines about how to use hyperlinks; they had absorbed hyperlink practices via observation, and when even when they coauthored in groups, they rarely had explicit discussions about hyperlink practices. Yet, nevertheless, the vast majority of authors I interviewed have developed relatively internally consistent practices, and, when asked, they could articulate reasons for their hyperlink practices. Those reasons, rather than being based in rules, were based in tacit understandings of what makes web writing different from print writing.

I began interviews with a goal of understanding hyperlink practices; through discussion, however, I discovered that the far more interesting story lay not in the rules or the practices themselves, but in the shifting allegiances reflected in the distinctions that authors drew between hyperlink citation style and traditional print citation style. People "just link"- but of course, those of us in writing studies know that no tacit practice "just" happens; because authors are developing these hyperlink practices as they go, their linking reflects their sense of online writing more broadly. Their link practices reflect not just what they have seen others do but also the way they sense online writing should be; their descriptions of why they engage in the practices they do reflect deeper-held

\footnotetext{
${ }^{38}$ I use the term "allegiance" to reflect the sense of ideological duty to these principles that I saw among participants, a sense of duty that supersedes pragmatic obedience to a style guide, something beyond the pseudo-economic exchange implied by a word like "value."
} 
assumptions about online writing. These allegiances indicate some of the things that are most important, most notable about Internet writing; they are the values of Internet writing that make it a distinctly different animal from what came before.

When I then turned back to analyzing online publications, with my list of allegiances, I realized that each of these allegiances is shared by most online texts that succeed in reaching wider audiences (within and outside academia); many publications that have not been successful in reaching wider audiences fail to reflect one or more of these allegiances. The problem, I argue, with most rhetoric and composition online publications that have ostensibly aimed at reaching wider publics, is that they have remained too allegiant to the values of traditional print academic text that I highlight in the previous chapter, and not allegiant enough to the values of web writing that I highlight in this chapter. Ultimately, I argue that we need a new kind of webtextual publication, one aimed at the public, aligned with the new allegiances of online writing and the necessary allegiances of academic publication, and designed with an eye to websavvy practices. In this chapter, I report on interviews with authors of webtexts and (more-)popular web writing (John Duffy, Anastasia Salter, Cate Blouke, Dawn Formo, Robert Kachur, Paul Muhlhauser, Kimberly Neary, and Daniel Schafer); and with webtext journal editors Doug Eyman, Cheryl Ball (both of Kairos), and Kristine Blair (of Computers and Composition Online). I rhetorically analyze writing by these authors in webtext journals and academic popular press. From this study of academic webtext citation practices, I develop my list of four allegiances of online writing: kairotic exigencies, availability, attention, and sharing. I relate how many webtext writers describe these allegiances and demonstrate how their texts reflect them. Current venues 
for academic webtext publication online, however, do not align with these allegiances; I report on how authors express frustration with the limited reach of online academic publishing. Ultimately, in the conclusion to this dissertation, I use these allegiances to develop a style guide for the kind of publication I am proposing, including but not limited to a "rhetoric of hypermedia" style guide.

\section{Hyperlinking Is Still Tacit}

I first became interested in allegiances when I realized that no interviewed author reported having been given explicit guidelines for hyperlinking. I expected authors to share "rules," either things they had been told about hyperlinking, or guidelines they had personally developed over time. Authors did not, however, have such choate understandings of when and why and how they should hyperlink. Writers I interviewed reiterated again and again: There are not yet standard practices for hyperlinking—web publications do not generally provide guidelines, recommendations, even suggestions for how or when to hyperlink (Kairos's warning about hyperlinking too much is, it appears, about as specific as guidelines get). Similarly, no authors described being given any explicit feedback on their hyperlink practices, from an editor, from other authors, from commenters or readers. Rather, authors described developing their own standard practices. Schafer, for example, described "collecting a corpus of examples in my mind and creating my own style guide, I guess, for how to do citation on the web over time." Kachur similarly described his first experiences with web writing, blogging professionally for a non-profit. In retrospect, he said, he was surprised by the freedom he had. In regard to hyperlinks, he was given "no guidance": "I kind of just watched, read, and learned." Cate Blouke - who worked for a couple of years as an editor of $\mathrm{Viz}$, the 
visual rhetoric blog of the University of Texas at Austin Digital Writing and Research Lab - explained that she has similarly had to invent citation practices as she has learned to write online and cite online sources; often, she said, "I don't know what the standard practice is, so I will make my own standard practice. This makes sense-let's do that. Anything you Google, like, 'How to cite this thing off the internet,' if MLA or Chicago even has an answer for that question, it's usually pretty clunky. But that's a big 'if,' often times."

Asking authors to articulate the thinking behind practices that have so far been tacit can help give shape to a series of standards-in-the-making. My previous chapters used data to get at the tacit, employing distant reading as a means of triangulating disciplinary assumptions. This chapter instead uses interview data to find disciplinary assumptions that have not yet been articulated, and to read those assumptions into the success/struggle of online academic writing. But more importantly—given my goal of proposing an academic web publication that reaches a wider audience, full of short-form, networked web-only opinion articles-I am especially driven by the pragmatic potential: This chapter is meant to pair with the style guide that follows it, as explanation and theorization of that practice-based document.

\section{The Rhetorical Velocity of Short-Form, Networked Internet Text}

The web-only opinion article, once so derided that "go blog about it" was a common put-down, has established a respectable cultural foothold. Most major news outlets have regular blog columns (e.g. the New York Times Well Blog), and the line between that blog content and the papers' "regular" content continues to blur. The webonly opinion article is a genre, I argue, that holds great potential for humanists to engage 
in the kind of international debate that some scholars (e.g. Mortensen, 1998) have argued is key to alleviating the crisis of the humanities. It has rhetorical velocity (Ridolfo and DeVoss, 2009) without sacrificing the potential for near-academic specificity. Blog-style and op-ed academic publications including The Chronicle of Higher Education (Chronicle), Inside Higher Education (IHE), and Reading the Pictures $(R t P)^{39}$ have all managed to reach a wide, transdisciplinary audience, often shared beyond the narrow bounds of academia — even though the Chronicle and IHE are quite explicitly aimed at an academic audience. A tale of academic insularity versus popular success, in three pairs of numbers:

- John Duffy's (2004) most-cited academic article-the College Composition and Communication (CCC) "Letters from the fair city: A rhetorical conception of literacy"-has been cited 24 times. But his (2013) Chronicle article, "The Ethics of Metaphor," has a total of $\mathbf{4 8 2}$ shares, likes, and comments on Facebook.

- Anastasia Salter's (2012) most-cited academic article—-the Journal of Broadcasting and Electronic Media "Hypermasculinity \& dickwolves: The contentious role of women in the new gaming public"-has been cited $\mathbf{6 0}$ times. But her most-popular Chronicle ProfHacker post of 2016, "Making Comics as Scholarship," has a total of $\mathbf{4 4 0}$ shares, likes, and comments on Facebook.

\footnotetext{
${ }^{39}$ Formerly BAGNewsNotes, a visual media analysis blog, which currently has three rhetoricians/compositionists on the list of regular staff/contributors: Christa Olson, Associate Professor of Composition and Rhetoric at UWisconsin-Madison, and Valerie Wieskamp and Philip Perdue, both PhD students at Indiana University.
} 
- Cate Blouke's $(2014,2015,2015)$ three academic publications have, as of yet, not been cited at all. ${ }^{40}$ But her (2011) most-popular BAGNewsNotes article, "ASU Students in Black Face Cheer on Football Team: Racist or Just Insensitive?" has a total of $\mathbf{6 8}$ shares, likes, and comments on Facebook.

Of course, I do not mean to suggest that Facebook interactions are equivalent to academic citations, in terms of significance or use; either can indicate a wide range of engagement levels, and a Facebook "like" can indicate friendly support of the person posting without necessarily indicating engagement with the shared material. Academic citations clearly demand higher involvement of the citing author, and they necessarily run at a slower timescale. In juxtaposing these numbers, rather, I am calling attention to the ways certain kinds of online academic publications have been successful at reaching audiences beyond academia, and to the ways that many academics in rhetoric and composition are already publishing in venues that seek and/or achieve reach an audience beyond the narrow confines of the field. The Chronicle, IHE, and RtP share many features that, I will argue, set them up for this public success. They are designed for circulation and uptake- - that is, they have rhetorical velocity (Ridolfo and DeVoss, 2009) — with single-page articles, a standard format, and practices that reflect the four allegiances I'll go on to discuss (kairotic exigencies, availability, attention, and sharing). These features are absent from much academic work published online, particularly work published in webtext journals such as Harlot, Kairos, and Computers and Composition Online; practices that do not reflect these new allegiances, I argue, limit the potential rhetorical velocity of much academic work online.

\footnotetext{
40 This is, of course, to be expected of articles published in 2014 and 2015; but the glacial speed of academic publishing is a major part of my point. It's not possible for academic articles to respond to one another rapidly.
} 


\section{Kairotic Exigencies, or Recency}

Several of the authors I interviewed argued that online writing has a new (or perhaps renewed) allegiance to recency, both in terms of citing recent events and ongoing public conversations, and in terms of publishing quickly enough to respond. Traditional academic publishing is an inherently slow process: Research must often be proposed, IRB-approved, and grant-funded. Prior work must be assessed, digested, and integrated. Data must be aggregated and analyzed. The scholarly product must be written and polished. Of course, all of that happens before the publishing time clock ticks on. In the sciences, many journals publish short-form "research notes" (and some journals ${ }^{41}$ publish only research notes), in which researchers describe current projects and relay preliminary findings. Humanities research - perhaps because it is so theory-driven, perhaps because its funding model does not as often demand that authors push findings quickly out the door to keep grants - does not, to my knowledge, have any journals that publish such a thing. 42

Some academics imagined moving scholarly journals online might speed up the publication process - no need to wait to correct proofs from the printer, to deal with binding and distributing. It turns out, however, that the social elements take much more time than the mechanics of publishing. Gérard Boismenu and Guylaine Beaudry (2004) explained that although the average time to publication for journals in Geoscience and Remote Sensing is 21.8 months (a typical timeline for journals in the humanities and

\footnotetext{
${ }^{41}$ For instance, BMC Research Notes and IO Journal.

${ }^{42}$ With the possible exception of the ERIC Digests, which provide summaries of educational research for the purposes of educators and policy-makers.
} 
social sciences, according to the authors), the actual mechanics of publishing account for under five of those months. Boismenu and Beaudry continued:

Publishing and disseminating an article in the digital world should a priori take significantly less time than is currently allotted for the process from a submitted manuscript to a printed, distributed article. However, when considering the process leading to the publication of a digital article and the estimated time needed, it becomes clear that a purely technical point of view obscures a significant number of the components of the process, components that are predominantly social. [...] In any event, just because we gain from the 'speed of machines' does not necessarily mean the 'slowness and vulnerability of human faculties' adopt a pace to match what is technically possible. That slowness and vulnerability are reflected in a whole series of manifestations from stretching activities out over time to the time it takes for reflection and writing a review. When we give a reviewer a month to provide an opinion on a manuscript, we know that a few hours are enough; however, we do not have any control over the expert's agenda or when he or she will do the job. (pp. 116-9)

Because distribution of digital academic work is nearly instantaneous, however, authors and readers expect its content to be more timely than print work. Boismenu and Beaudry suggested that readers' "paradoxical values" of carefully vetted and timely content will likely remain a perplexing challenge for online academic journals; "vetted" and "timely" seem to sit at opposite ends of a spectrum, and the peer review process pushes publications constantly toward "vetted." 
Yet web culture seems to push in the other direction. It has been almost twenty years since Jakob Nielsen (1999) warned, "Web users are getting more impatient every year." In the Netflix political thriller House of Cards (Treem, 2013), a young reporter leaves a newspaper of record, the Washington Herald, to write for an upstart Politico-like web publication, Slugline. After years of resisting an editorial machine that slowed her scoops, she is thrilled when her new boss says, "You don't have to send me things before you post. The goal here is for everyone to post things faster than I have a chance to read them. If you're satisfied with the article, just put it up. Whatever hoops the Herald made you jump through, let them go." The show dramatizes the binary predictably, the Herald's drab cubicles set starkly against Slugline's energy drink-chugging twentysomethings draped over bean bags, laptops perched on knees. Vetting is for old people, the scene suggests, and the Internet moves too fast to bother with it. For a reporter like Zoe Barnes, who aims to affect government policy by reporting on political action as-ithappens, such a release from careful editorial vetting could make the difference between a story worth reporting and a story that bobbles at the starting gate.

Though the rapidity of Internet publishing may make readers' thirst for timeliness more appreciable, the importance of deploying an argument at just the right moment is perhaps one of the oldest ideas in rhetorical theory. In fact, Kinneavy and Eskin (2000) demonstrated that kairos was even more deeply interwoven into Aristotle's definition of rhetoric than previously thought, pointing (for example) to a moment in the first chapter of Rhetoric in which Aristotle described the necessity of urgency in "rhetorical decisions in the assembly or the court" (p. 435). Kinneavy and Eskin continued, "This concept thus repeats Plato's (trans. 1927) notion in Phaedrus of the theoria-praxis distinction, which is 
mediated by kairos [...] The notions of usefulness, expediency, and suitability are all situationally determined" (pp. 436-7). Kairos connects an argument to its situation and determines the appropriate use of theory. Carolyn R. Miller (1992) has similarly argued "for the centrality of kairos to rhetoric. As the principle of timing or opportunity in rhetoric, kairos calls attention to the nature of discourse as event rather than object; it shows us how discourse is related to a historical moment; it alerts us to the constantly changing quality of appropriateness" (p. 310). That is, when you say something mattersas both the ancient rhetorical greats and fictional political reporters have recognized—at least as much as what you say or how you say it.

When hypertext originally began to gain cultural significance, some theorists suggested that it was flattening time, eliminating the importance of timeliness. Nearly two decades ago, for instance, Manuel Castells $(1996,2000)$ worried that hypertext was pushing the world into a "temporal collage, where not only genres are mixed, but their timing becomes synchronous in a flat horizon, with no beginning, no end, no sequence" (p. 492). He postulated that as information moved online, where information is ordered "according to the impulses of the consumer or to the decisions of the producer" rather than according to meaningful social context, all information becomes acontextual and achronological. Yet computer-mediated communication has not made chronology obsolete. In fact, the rise and ubiquity of social media has collated hypertext into an eternally refreshed series of reverse-chronological feeds and timelines, where recency is regent. Consider that web analytics researchers (Kissmetrics, 2014) have pinpointed the best time to publish an English-language blog post for maximum readership-Monday morning at 11 am Eastern Standard Time, when the highest percentage of the blog- 
reading United States workforce is at their desks and caffeinated. And lest one fear (or hope, perhaps) that this eternal refreshing has created an entirely ephemeral culture, consider the popularity of so-called "nostalgia apps" such as Timehop, which invite social media users to revisit and republish posts from years past.

Rather than chronology being flattened into a vast expanse of textual timelessness, textual time has become measured in increasingly minute increments: Now, most things published online have a publishing time identified down to the minute, sometimes even the second. Many sites (including the text-sharing site Medium) warn readers how long an article will take to read, in minutes. Read receipts accompany texts and chat messages and emails, so computer communicators know when their communique has been acknowledged. Yet few online academic journals in rhetoric and composition have followed suit: Editions of Computers and Composition Online are measured in half-year academic increments ("Fall 2016," "Spring 2015," etc.). Kairos, too, publishes an annual Fall and Spring issue, and sometimes an extra Spring or Summer special issue. This is time measured as academics do. Harlot was even less discrete, with editions identified only by year and an issue number. And none of these online academic journals with an ostensible public aim appear connected to a single chronological moment in the way that is common among so much online text. In failing to adhere to this sign of the times (by signing their times), they reflect the glacial academic publishing timeline, and simultaneously, I believe they miss out on a significant advantage of online publishing: A sense of universally distributed in-timeness not yet possible beforehand. 
Bowling Down a Mile-Long Lane: "Connect the Things We Know to Kairotic Moments"

By limiting academic web publishing to peer-reviewed venues that have traditionally long times to publication, we squander a significant potential affordance of online publishing: Timeliness, one of the most important available means of persuasion. Of course, finding the right moment for an argument requires that the rhetor understand the rhetorical situation, have a clear purpose, and be able to take advantage of the moment. Further, as Miller (1992) pointed out, "right" is highly contextually dependent: "If a particular moment in time is right for some action, the next moment, while wrong for that action, is right for another" (p. 312). Yet a twenty-month gap between writing and publishing leaves writers forever penning scholarly communication at a moment when it might have been right and releasing it to an audience at a moment that is much less likely to be right. Attempting to hit a kairotic moment twenty months out seems like throwing a bowling ball down a mile-long lane: No matter how hard and true your throw, a shift in winds or poorly waxed spot could easily turn it into a gutterball. Any text that happens to arrive in a newly appropriate kairotic moment—such as Paul Walker's (2016) Rhetoric Review article that happened to consider the construction of British nationalism at the exact moment of the Brexit (see the previous chapter)—risks appearing tone-deaf, unaware of its mile-long kairotic strike.

A model for how rhetoricians might take advantage of faster publishing times is John Duffy's $(2012,2013)$ work in IHE and the Chronicle: The former responded to the moment when Rush Limbaugh used words including "slut" and "prostitute" to describe a Georgetown Law student who spoke to a House committee about contraception, using that controversy to argue that universities must instruct students in more-ethical 
discourse. The latter related Sarah Palin's description of the US-China relationship as being "like slavery," as a springboard for developing a heuristic for assessing whether a metaphor is ethical. Finding relevant current examples was key, Duffy said, both for him and for the editors at IHE and the Chronicle:

I actually wrote it ["Virtuous Arguments"] in response to just general arguments about civility and then Rush Limbaugh made his statement. I revised it, and then it got published. One of the things that the editor said was the timeliness of it was really important. I think that's something to take away. If we can connect the things we know to kairotic moments in public discourse, in popular culture, in the media and so forth, we stand a better chance of getting published.

In addition to the Limbaugh reference, Duffy provided several other examples of public figures behaving uncivilly, each with a link to an online article discussing the offense (an article about Glenn Beck from The Daily Kos, an article about Maxine Waters from Fox News, and so on). He said he spent considerable effort searching for and selecting relevant examples, to balance the paragraph with right- and left-wing offenders. This wealth of link citations ${ }^{43}$ exemplifies both link chaining and the materiality of the underline, as I defined each in the "rhetoric of hypermedia"; the links' quick succession and underlined roster of famous names demonstrate that this is an ongoing recent phenomenon and that people you've heard of are involved — without having to provide more than a sentence of background or additional context for any example, because

\footnotetext{
${ }^{43}$ One intriguing observation on anchor text: Duffy's $I H E$ and Chronicle posts reflect very different anchor text practices. The IHE piece has hyperlinks over examples' names only (e.g. “...such as Glenn Beck, who once mused on-air..."). The Chronicle piece puts hyperlinks over phrases, sometimes linking an example's name and verb (e.g. "...former Republican vice-presidential candidate Sarah Palin stated that when...") and sometimes linking a verb and object (e.g. "...Florida Democrat Alan Grayson compared the Tea Party to the KKK..."). I was excited when I first realized this, because I thought Duffy was an exception to my rule that authors develop tacit, internally consistent linking practices. Except our interview revealed that he didn't place the anchor text-he just gave the editors a list of links, and the editors linked up the pieces. Duffy was the only author I interviewed who did not place his own links.
} 
Duffy can be sure that any readers who need or want that context can simply click through.

Duffy's second piece, while also connected to some specific examples in public discourse, responded at the same time to a more personal kairotic exigency Duffy felt, a question from a student:

It was very much the same with the second piece [...] I was noticing all the KKK references that were coming about, and at the same time, I had been asked to speak to Right to Life Club at Notre Dame. [...] One student asked what I thought was a really great question: 'What kind of criteria do we use to determine whether a metaphor is ethical or not?' I gave some really weak answer. [...] So I went back and thought about it for a day or two and then wrote that student a letter. That letter was the basis of the article. I adapted it and sent it off to the Chronicle.

The encouragement about including contemporary examples from the IHE editors when he published that first semi-public text apparently influenced this second text, however. Although Duffy reported both personal and public exigency for the article, his final text only addressed the public — creating the sense that it was an extemporaneous response to Palin's "slavery" metaphor. As in the $I H E$ article, the controlling example is followed by a string of other recent examples of inappropriate, appropriative slavery and KKK metaphors. Consider how different the text might have been had he presented it as an open letter to the student rather than an op-ed; the relevance of the argument to societykey, of course, to Duffy's message—would have been significantly more oblique. The genre of the web-only opinion article offered a platform for an argument that would have made far less sense by the time it could have been published in a traditional print journal. In the twenty-month publishing hang time, the kairotic moment of racially charged metaphors would likely have passed, and Duffy would have had no opportunity to edit the piece, to add more timely examples. 
Kris Blair spoke similarly about what she termed the "rhetoric of immediacy,"

when she described a chapter of a book she was working on that drew on web sources:

Because it related to gender and technology, so much of what I wanted to talk about was either in books - published books - or online, in just the horrors of things going on in the IT industry and the Gamergate. So when I wanted to talk about those issues, I often would just be looking at articles online - Cory Doctorow, looking at things in Newsweek, different sorts of online spaces. I totally think there is that tendency. And going back through it, [...] I wonder if people are going to think this first chapter isn't academic enough, because it's kind of - I have to admit it, and I don't normally say this about my writing-I thought it was really fun to read [...] because it was so current and relying on so many examples that are happening as of last week. And I think that's the issue that makes web-based publishing and that reliance on more readymade primary texts like that so easy and so appropriate.

Even though Blair presented her concern that her chapter might be read as "not academic enough" in the context of her research practices, I was still surprised when she suggested that citing recent Internet sources might of itself make such an established scholar-an editor of one of the leading webtext publications in the field, no less- be read as unacademic. Interesting, too, is her assertion that by including these recent examples alone, she has made the text more enjoyable to read; at no point did she discuss changing, for example, her prose style or organization.

Intriguingly, Blair also described having, in that book, a list of online resources for other scholars; the reviewers, including apparently Cheryl Ball, asked her to remove the resources - the links will die, they said, and you have to consider the shelf life of the book compared to the shelf life of the websites. Immediacy is limited in traditional academic publications by research times, by writing times, by review times, by revise and resubmit times - and then, eventually, by the few weeks to few months that it takes to get something typeset. Online publications are not necessary able to avoid the first long list 
of things; many academics working online have found that it takes essentially as long for a piece to go from conception to publication. I do not think traditional academic journals should give up their long publishing times; after all, that plodding thoughtfulness results in carefully considered work, work with well-considered thought, vetted by other experts in the field, revised with an eye to making a contribution to that field. Rather, to take advantage of the potential rapidness of Internet publishing, an online publication needs to eliminate or rapidly condense the review and revision process, to condense writing times by preferring shorter pieces and less thorough investigations. Long-form text has its place; traditional peer review has its place. Yet the academic Internet in rhetoric and composition is oddly short of publications that take advantage of the rhetoric of immediacy.

Academic Publications Increasingly Include Internet Sources

If even the editor of one of the leading webtext publications in rhetoric and composition is concerned that citing electronic sources will make her work appear less "academic," should the rest of us have similar worries? I was reminded of Slate author Rebecca Schuman's report (from Chapter Two) that some academics had criticized her for making "unsourced" arguments, a particularly strange complaint in light of my data that demonstrated Schuman cites more than three times as often as the average Slate article. Clearly, there are yet some scholars who dismiss web sources as not academic enough. How widespread is that dismissal? Likely less every year, my data suggest. To get at the question of the discipline's reception of web sources and webtexts, I returned to my $C C C$ database from the previous chapter and wrote some analysis programs: The first of these programs flagged every article that included the word "webtext," "website," or 
"Internet," simply to get a broad picture of the extent of the field's conversation about the Internet (see Figure 4.1). For a point of comparison, we can look to Doug Eyman and Kairos Praxis Editor Colleen Reilly's (2006) analysis of citation of online works in CCC, CE, Journal of Advanced Composition, and Computers and Composition from 1996 2003. They discovered "that very few articles in these print journals cited any kind of electronic resource" - in fact, just 3.5\% cited any electronic sources. ${ }^{44}$ Of course, my bar was lower than theirs; looking for "website," "webtext," and/or "Internet" surely returns some articles that only offhandedly mention the information superhighway. Nevertheless, the upward trend is clear: In 2014, nearly three times as many articles showed signs of Internet life as in the last year of Eyman and Reilly's data (52.2\% of 2014 articles, compared to $17.8 \%$ of 2003 articles). 2014 was also the first year that the majority of $C C C$ articles used one of those three words. This graph suggests that perhaps Blair's fears are unfounded, at least to the extent that referring to the Internet in academic work in rhetoric and composition is now the norm, not the exception.

\footnotetext{
${ }^{44}$ With more time, I would replicate this study more exactly. Unfortunately, I discovered that it is not feasible to consistently separate the References list from the rest of the article text, and when I searched for URLs, I got too many false positives.
} 


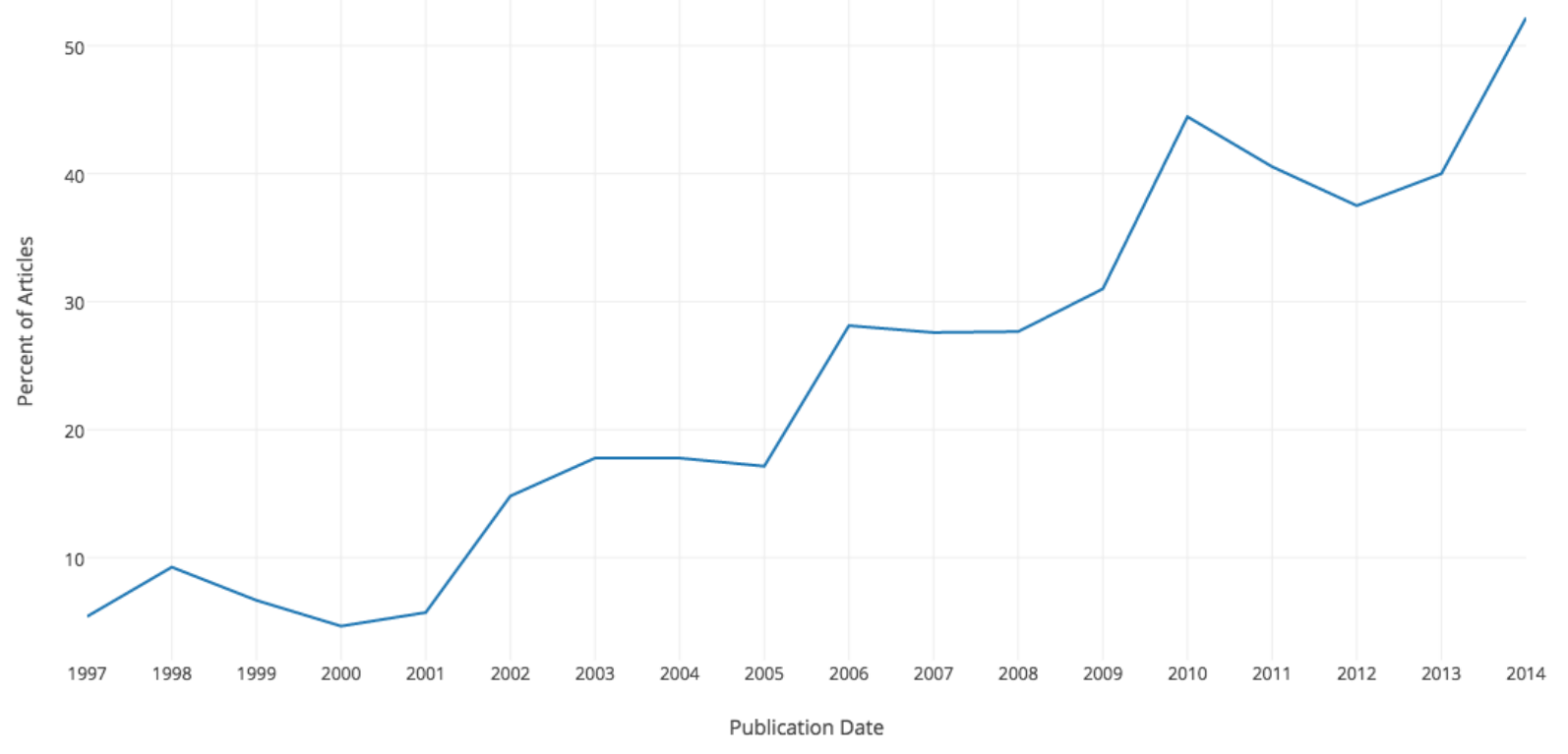

Figure 4.1: Percent of articles in $C C C$ using the word "website," "webtext," and/or "Internet," each year 1997 - 2014.

To get a sense of how the field has responded to online journals more generally, I ran another program that flagged every article that included an in-text reference to a webtext publication's URL or name, using a wide selection of webtext publications current and defunct ${ }^{45}$ (see Figure 2). Again, these data are promising, in terms of the generally rising disciplinary prominence of webtext journals. There is more ebb and flow here-1997 and 2011 are at an identical $10.81 \%$ of articles — but in 2014, over a third of CCC articles nodded to a webtext journal.

\footnotetext{
${ }^{45}$ I searched for the following phrases: "kairos.technorhetoric.net", "Computers and Composition Online", "bgsu.edu/cconline/", "Harlot of the Arts", "harlotofthearts.org/", "Enculturation" (capitalized as such), "enculturation.net/", "enculturation.gmu.edu/issues/", "Present Tense" (capitalized as such), "presenttensejournal.org/", "Composition Forum" (capitalized as such), "compositionforum.com/”, "Across the Disciplines" (capitalized as such), "wac.colostate.edu/atd/", "English Matters" (capitalized as such), "englishmatters.gmu.edu", "Journal of Writing Research", "jowr.org", "RhetNet", "wac.colostate.edu/rhetnet", "Rhizomes: Cultural Studies in Emerging Knowledge", "rhizomes.ent", "The Writing Instructor", "writinginstructor.org", and "parlormultimedia.com/twitest/". Note that I excluded the name "Kairos" because it resulted in too many false positives.
} 


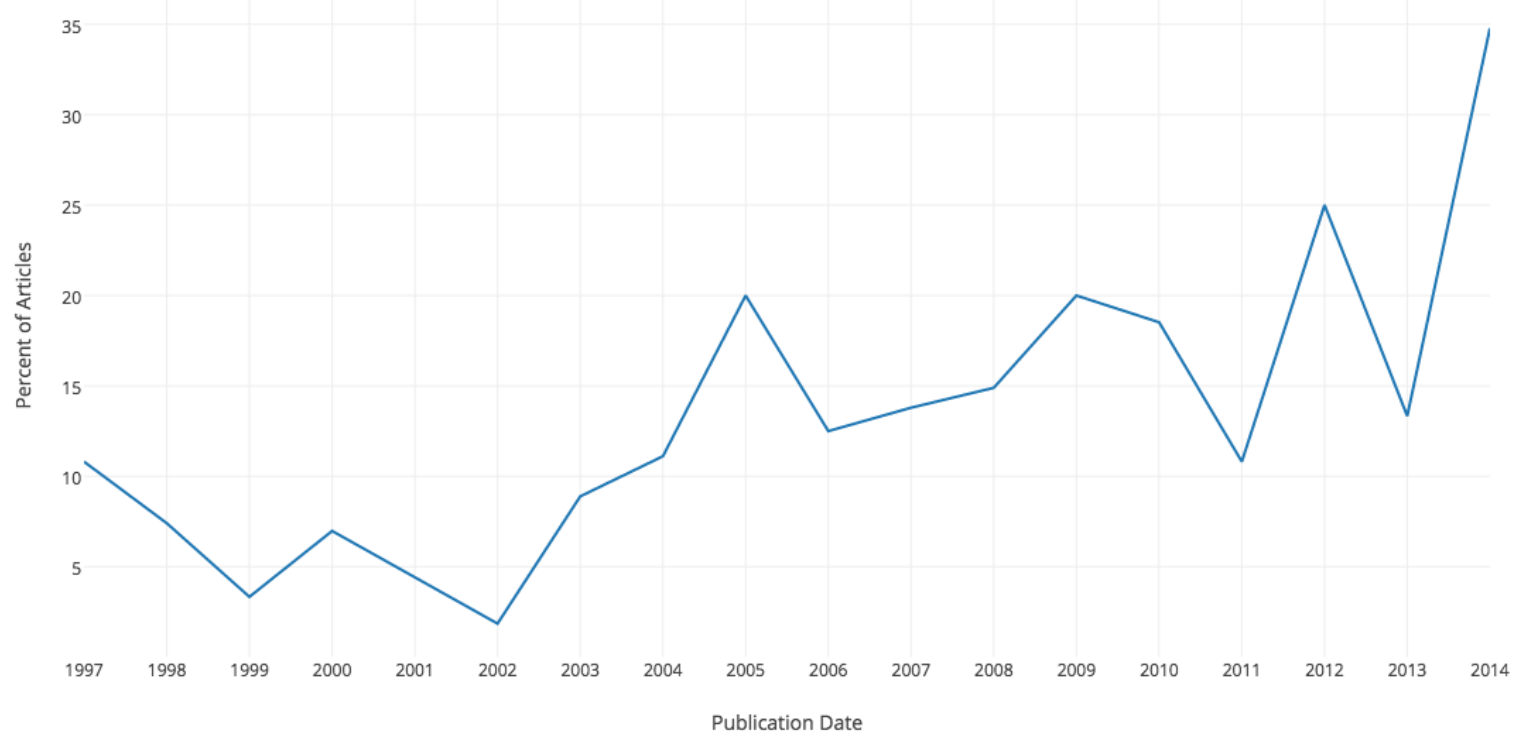

Figure 4.2: Percent of articles in CCC including the name or URL of an online rhetoric and composition journal or to a website (see footnote 4 for a detailed list of included search terms), in $C C C, 1997$ - 2014.

Nevertheless, articles in Kairos have a much smaller reach to the general publicas measured by Facebook shares/likes/comments - than articles published in the Chronicle (which, again, does not explicitly aim to reach the general public). To explore this question, in May 2016, I requested share/like/comment data from all the articles in the September 2015 issue of Kairos and all the articles in the Chronicle from the same date. On average, Kairos articles had 43.2 shares/likes/comments; on average, Chronicle articles had 541 shares/likes/comments. In the Kairos issue, the peak share/like/comment value for a single article was a total of 329 (26 shares, 262 likes, and 41 comments), for Amanda Fields, Londie T. Martin, Adela C. Licona, and Elizabeth H. Tilley's (2015) "Performing Urgency: Slamming and Spitting as Critical and Creative Response to State 
Crisis," which reports on the Tuscon Youth Poetry Slam. By comparison, the peak for a single article on the Chronicle was more than an order of magnitude larger, a total of 3,693 (700 shares, 1,979 likes, and 1,014 comments), for Steve Kolowich's (2015) "Is Nuance Overrated?"- which, curiously, reports on an interview with sociologist Kieran Healy, who argued for bolder, less equivocating sociological theory.

Kairos and the Chronicle share a portion of their intended audiences: Academics who teach, research, and are interested in publishing articles for other teachers and researchers. Yet the Chronicle operates very differently; it publishes several posts a day, by authors from across the university. It publishes on a rolling, magazine- or newspaperlike time schedule, rather than in semi-annual, academic chronology-based issues. It publishes short-form articles, generally in the 600- to 2000-word range, common among this kind of short-form networked web opinion journalism. And every posts identifies not just the day it was published but the time, and comments below the article are organized in a chronological feed, each comment labeled with a to-the-minute timestamp. Further, most posts in the Chronicle make an attempt to connect themselves to an event or a recent, ongoing discussion. The Chronicle is thus responsive to kairotic exigencies in a way that Kairos, despite its name, is not. There are other differences between the journals, too, of course-Kairos is aimed at rhetoric and composition scholars particularly, where the Chronicle is interdisciplinary. Kairos publishes webtexts, where the Chronicle publishes op-eds. Kairos is peer-reviewed, where the Chronicle is edited. Kairos is fully open-access, where the Chronicle is "freemium" or partially paywalled. My point is not that Kairos should be more like the Chronicle. Rather, I am suggesting 
that if we want to develop a publication that is more responsive to kairotic exigencies, we would be better off modeling on the Chronicle than Kairos.

All of this is to say: Online writing has a particular allegiance to the notion of kairotic exigency. Because things get published quickly online, readers seem to look for evidence that a text is connected to the wider hypertext of the Internet. Though there is increasing acceptance of web sources — both academic and not — as potentially valuable locations of research, Kairos, somewhat ironically, is less able to be kairotic about its web citing than a publication with a faster publishing schedule such as the Chronicle.

\section{Availability Matters More than Stability}

Although the continued stability of webtext journals is, understandably, important to the editors who keep those journals running, the authors with whom I spoke generally expressed far less concern about the stability of webtexts than I expected. Hence, in this section, I will argue that an allegiance to stability is, in the minds of many web authors, superseded by an allegiance to availability of shared resources. I will elaborate on the distinction between available and stable at length below, but briefly, availability puts the focus on a resource-in-use, whereas stability puts the focus on the resource-as-object. Authors seek resources that are available to the broadest possible swath of readers, presented in a way that makes those resources available at the moment of need. To explain why this shifting allegiance to availability is noteworthy, I will begin by considering how and why a focus on stability has been such a key feature of successful academic web publishing.

The notion that text itself or writing itself is unstable is endemic to the postmodern humanities; we are past the death of the author (a la Barthes), we recognize 
the idea of an author itself to be unstable (a la Foucault), and we accept that all context is part of the text we must analyze (a la Derrida). We talk as often about genres and systems as we do about individual texts. We recognize that the whole Internet, maybe even all text ever published, is at some level a hypertext, all one mass of interconnected, mutually dependent writing. Thus, when I say that academic writing, even online academic writing, has nevertheless held a value of stability, you might reasonably take umbrage at the suggestion. My invoked audience cries: But what about all the nonlinear hypertexts on Kairos? But what about the rise of alt-dis (and here you might cite Bizzell, Schroeder, and Fox, 2002)? Haven't we, in our PoMo fervor, redefined everything from "text" to "hypermedia" to "composition" ("and beyond," you might say, citing the likes of Johnson-Eilola, 1997; DeWitt, Strasma, \& Farr, 2002; Rhodes and Alexander, 2015)? Yes, we have troubled the concept of stability quite broadly. Yes, we have come to recognize, as Thaiss and Zawacki (2006) argued, that academic writing itself "is not as stable, unified, and resistant to alternatives as we often theorize it to be" (p. 2). Yet there has been, nevertheless, a sense that academic objects published online should be, like their print brethren, meant to exist in perpetuity, remain stable for readers twenty, forty, a hundred years on. I wish, in this section, to trouble the notion that academic texts online ought to remain stable, and instead to argue that to make the best use of the affordances of web writing, we ought to embrace its necessarily more fleeting, more transient, and more unstable nature. Text itself is no longer assumed to be stable, assumed to stay the same from website visit to website visit. Who today would put an "Under Construction" image on a website, as many web authors did in the late 1990s? Of course, it is out of the aesthetic vogue — but more importantly, all websites are now assumed to be "Under 
Construction." A website is expected to change from visit to visit, to offer new and continually interesting content. And often, also to allow users to contribute to the content of the site, via Web 2.0 tools. Stability is anathema to a site populated in part or whole by user-generated content.

I entered interviews with awareness of an ongoing conversation about the problem of "link rot" in online educational materials, the way that web content has a tendency to change addresses or disappear entirely, leaving visitors to old links with a 404 error. Katelyn Burton (2015) worried recently in Computers and Composition about the "digital gaps and losses [that have] become ever more alarming" as digital pedagogies become commonplace; Burton pointed to the first issue of Kairos, which includes Paul Johnson's (1996) exploration of online writing labs, in which 30 of the 31 hyperlinks have gone dead. "Though Johnson's message," Burton explained, “composed in text, still exists in its entirety on the Kairos site, the context of this message, originally illustrated and conveyed through what we might assume to be various OWLs and other scholarly discussion of OWLs, is all but lost." Quantitative analyses of link rot suggest that this problem is by no means limited to rhetoric and composition journals; it is endemic to the web. John Markwell and David Brooks (2003), for instance, discovered in an assessment of links in a series of online biology and biochemistry courses that a full $20 \%$ of links were dead or otherwise inaccessible (e.g. behind a paywall) within two years. David Tyler and Beth McNeil (2003), in an analysis of links published in the College and Research Libraries News between 1994 and 2001, similarly found that links persisted an average of between four and five years - and counting redirects, over six (p. 620). 
Yet while Burton presented her argument against what she termed "rampant myths of permanence," my interviews with webtext editors suggest that awareness of the potential, maybe even inevitable impermanence of digital publishing weighs heavily on the minds of people most embedded in it. Given the recency of digital publishing as a phenomenon, its veterans are still running the show—Doug Eyman has been working for Kairos since its second issue - and they are plenty aware of the impermanence Burton (2015) pointed out. Eyman described how, in the early years of Kairos, authors were encouraged to keep making changes/emendations to their webtexts after publication, often actually hosting the "main" webtext on their own servers, with Kairos maintaining a second, back-up copy that reflected the text just after peer-review. Yet this caused several kinds of problems, Eyman explained to me:

There was no real reason to have these two versions, and there was a real strong disincentive, in the sense that sometimes, early on, the authorized archived peerreviewed version didn't get to the server. So when the authorial version went away, the whole piece was gone. That leaves gaps. That was an early lesson in, 'If we can't archive it, have the archived copy be the main copy, then we can't publish it,' because we have to keep our archive sustainable.

Practices of Kairos developed in response to such lessons have helped it stick around, far beyond the tenure of most online (and even many print) journals. The continued existence of journals such as Kairos and $C \& C O$ has made a vision of stable academic web publishing a reality, where it did not exist before. It is understandable, in the tumultuous context of the rise of web publishing in rhetoric and composition, that such journals would feel particularly compelled to stress to authors the measures they take to make work exist consistently and in perpetuity. 
I do not mean to downplay the significance of stability for the purposes of webtext journals such as Kairos and $C \& C O$. If academic web publishing is to "count" as scholarship, either for tenure and promotion purposes, or in the more general sense of contributing to an ongoing scholarly conversation, webtexts must stay online, in a predictable location; unfortunately, web authors in rhetoric and composition have learned this through bitter experience. The example of CCC Online, for instance, is still fresh in the minds of many rhetoric and composition scholars; the "journal" (unified only roughly in name and sponsoring organization) has come and gone three times, each time shorter than the last. Pieces published in the first version of CCC Online (2002), which aimed to publish Internet-native webtexts, are now available only via web archiving services. Authors were not informed prior to the shutdown of that first CCC Online; Steven Krause - who published an article in that issue somewhat ironically titled, "Where do I list this on my CV?" (2001/2002)—described in a follow-up Kairos article (2007) how "one day the article was available at the site [...] and then one day it was not." The second version of CCC Online had a different vision entirely: Collin Brooke and Derek Mueller were contracted to write abstracts and develop tags for all $C C C$ articles back through 1987 on a website (inventio.us) that, while still available, has not been updated since $2008 .^{46}$ The third and most recent iteration (2012) lasted just one issue, was only

\footnotetext{
${ }^{46}$ According to a post on Brooke's blog (2008) tellingly titled, "Exactly who's been in charge for the last 10 years?," CCCC higher-ups informed him and Mueller in Fall 2008 that their work at inventio.us was being renamed from CCC Online to CCC Online Archive, without further explanation; then, a few weeks later, CCCC posted an advertisement looking for a volunteer "CCCC Web Editor." "What is most insulting," Brooke (2008) explained, "is that my title was changed as part of a conversation to which I might have had something to contribute, and to which I almost certainly should have been invited. As I said, I first saw the ad yesterday, the day before the deadline." The CCC Online Archive project stopped updating soon after. Meanwhile, at NCTE.org, "The Extended $C C C$ " continued to publish full versions of essays that were only represented with abstracts in print. The impolitic nature of the announcement aside, the saga created sufficient confusion that nobody I interviewed - not even Alexander, the current editor of $C C C$-would confidently assert a history or timeline of CCC Online ventures.
} 
available on Dropbox, and - according to Jonathan Alexander, who wrote a webtext for it — remains a sad topic for the authors who put significant effort into their pieces for it.

Kairos, $C \& C O$, and other web publishing venues such as the Computers and Composition Digital Press have attempted to introduce predictability and stability to rhetoric and composition's web publishing. Nevertheless, even $C \& C O$ 's tenure has been somewhat tumultuous: The journal changed web addresses twice recently, once in 2015 (from www.bgsu.edu/cconline to casit.bgsu.edu/cconline, where only the most recent articles were available, no archives) and once in 2016 (to http://cconlinejournal.org/). Though $C \& C O$ will likely remain at its new home, all three editors I interviewed expressed anxiety about the change. When I spoke to Kris Blair soon after the 2015 web address change, she explained that, although she had updated the $C \& C O$ social media feeds and attempted to alert as many people as possible, many others were still emailing her asking why the journal was “down.” Doug Eyman recounted in our interview that he discovered the $2015 C \& C O$ change through emails from Kairos readers-who wrote to alert him that all of Kairos's links to $C \& C O$ were out of date. Kairos is likely one of just a few journals that have made a concerted effort to correct out-of-date links to $C \& C O$, and with the change to a hopefully more permanent URL, many links are out of date yet again. Cheryl Ball named many efforts she has made in her time as Kairos editor to establish regularity and consistency in publishing. She introduced, for instance, a timed series of eight copy-editing stages, documented in great detail on a private wiki site, through which each text passes; thus, an issue with nine texts will undergo 72 separate copy-edit reviews, via a team of more than 30 full, associate, and assistant editors (myself included). The Kairos style guide's admonition against hyperlinks, Ball said, is one of 
these stability-focused practices. Whenever you link out, she explained, you run the risk that "the link dies, and then you've linked to the 404 message of doom, the abyss of the internet, and then you [the reader] have to try to find the thing if you're really interested in it." Working to provide the sort of web publishing experience that authors can present as equivalent to print publishing in both seriousness and longevity, editors such as Ball, Eyman, and Blair understandably value stability—after all, in the short history of academic web publishing, most journals have been anything but stable.

Yet while editors expressed such concerns with stability, many of the mostprolific webtext authors I spoke to were not deeply concerned with the idea that the links in their online writing would likely break soon. Like Ball, many described this as simply the way of the Internet. Instead of suggesting it is a reason to avoid hyperlinking, however, many pointed to the copious ways content behind "dead" links is often still available. Some actually seemed to appreciate the ideological implications of web publishing's ephemerality. Anastasia Salter, for example — who was likely the author with the most web-writing experience I interviewed—said that, given the wide availability of web archival services, she was unconcerned about "link rot." She further suggested that often, links go dead as their content is no longer relevant:

I'm not worried about that at all. A dead link is just an invitation to go to the WayBack Machine and put in the link. I'm much more concerned about paywalled links, which to me are the equivalent of dead ends. If I'm citing an article that you have to have an extremely expensive institutional subscription to read, then I can link you to it, but unless you're within our particular set of institutions, then you're not going to be able to follow that path, so it's not a useful path. I don't find the dead link concern to be all that relevant. Most academic journals keep their online archives, online websites fairly consistent. At the same point those things die, they're irrelevant, too. We're far too concerned about permanence, in what's weirdly mostly a fleeting pursuit of location in scholarship. 
Salter's primary concern is not so much that things stay where they were at the first happening upon but rather that they stay available for people to consult later-and for a wide range of people to consult, in all kinds of situations. As a writer for the Chronicle ProfHacker column, Salter is acutely aware that even within academia, people have varying levels of access to most paywalled academic journals; most academics can recall the frustration of finding "the perfect" article abstract in a bibliography or via a library database, only to discover that the sought issue is unavailable. And as Salter pointed out, automatic web crawling archival tools such as the WayBack Machine ${ }^{47}$ are far more likely to have a copy of a 404'd website than non-academics are likely to be able to track down paywalled academic content.

Paul Muhlhauser echoed this sentiment when I noted that a couple of links in his recent (2015) co-authored piece, "May the \#Kairos be with you," were already dead.

Choosing to include hyperlinks, he explained, was a somewhat novel practice for him:

OK, so I used to be really in the past. I was like, 'OK, I've got to have everything in my one folder. I want it to last forever.' So I would collect everything. I would put it in there. And then it goes online, it's its own thing. You've got your web folder, all that's in there. With this piece, I was like, 'You know, I really like adding this extra element [of hyperlinking] to it. I'm not going to worry so much about it.' But as I reflect back on it, there are ways to [preserve linked content] that are pretty easy. I do want those links to still exist. [...] I think there needs to be something like this at journals: BrowseShot. That's an easy way to collect a whole long page. It puts it in JPEG. [...] In the future, it seems like this could be how you archive things. There's still the [Internet] Archive that exists. But sometimes that's incomplete. So why not just click that button? Get it.

\footnotetext{
${ }^{47}$ A tool run by a 501(c)(3) non-profit called the Internet Archive that also provides-among other things - an enormous collection of creative commons-licensed media, the Open Library Project, and the Prelinger Archive. Doug Eyman pointed out in our interview that the Internet Archive is potentially manipulable by ill-intentioned government or corporate agents. When a "robots.txt" file (a short text file with a "disallow" instruction for web crawlers) is added to a website, he explained, the Internet Archive will retroactively obey it for all pages. Anyone wishing to shut down an archive of a site with objectionable content need only gain server access and leave a "robots.txt" file somewhere.
} 
Like Salter, Muhlhauser emphasized the value of web archival tools and suggested that the continued existence of the site linked to was immaterial — provided that the content was still available. I was especially struck by Muhlhauser's characterization of having all the resources of a publication available in the same web folder as "in the past." On reflection, I was briefly baffled: Why would having all your resources in a web folder be an outdated attitude? Then I considered the meteoric rise of external hosting services. Videos, images, sound, and even some kinds of text are now frequently hosted off-site, with providers such as YouTube, Imgur, Soundcloud, and Scribd, which offer fully interactive embeddable objects. There appears to be growing trust of external media hosting; increasingly, websites seem to prefer external hosting over hosting their own videos. Even webtext journals, focused on stability as they are, now host most of their videos off-site (Kairos, for instance, displays video and audio on the site via YouTube embeds, but also stores local backup copies of all files, in case of a YouTube apocalypse). The chance that YouTube might disappear into the ether seems much lower than the chance that visitors will not have an up-to-date version of the necessary plugin to play a .wmv file - and thus, this is a prime example of the shifting allegiance from stability to availability. To make videos available to a wider group of potential viewers, many if not most websites now give up the stability afforded by self-hosting for the availability offered by external hosts.

Importantly, stability versus availability is not a zero-sum game, and I do not mean to construct a false binary: Choosing to host videos on YouTube does not appear, at the moment, to be an especially unstable option. More than ten years after YouTube's founding, it does not seem in any danger of disappearing. However, anyone seeking 
reminders of its instability need only upload a video with a few seconds of music under copyright; YouTube's automatic piracy-prevention bots will remove the video within minutes - and YouTube retains the right to remove any content they deem unsuitable. After the 2014 Isla Vista massacre, for example, several videos posted by the killer began to gain traction in mass media, until YouTube removed them for "violating community guidelines" (in the process, breaking the embedded videos in many relevant news articles). Yet, most web authors are willing to accept the possibility that externally hosted content may be removed at any time, in exchange for those hosts' availability and nearuniversal accessibility.

Muhlhauser's characterization of link rot worry as anachronistic seems supported by the fact that even authors who had never before written for the web, on a blog or otherwise, expressed a similar sentiment. Kimberly Neary, who along with Dawn Formo, was writing for the web for the first time in their Fall $2015 C \& C O$ piece, said she and Formo had been aware of but not terribly concerned with the potential for link rot. Like Muhlhauser and Salter, she named the WayBack Machine as a readily available recovery tool. ${ }^{48}$ She described the thought process behind their decision to link to external resources including a video produced by Nancy Sommers (2002) and published by Macmillan:

I think we chose pieces that we felt were stable-ish. [...] Nancy Sommers's piece, I mean, she's a name in our field. Do I imagine that 'Beyond the Red Ink' is going to vanish any time soon? Does this [potential that the video might disappear] give some sort of temporary aspect to a piece that we hope is not

\footnotetext{
${ }^{48}$ Though the WayBack Machine is certainly the most famous and widely used web archival tool, there are several others with good range, relatively solid support, and a similarly wide history. A service called Memento aggregates a bunch of them into a single search called "Time Travel Find." Screenshots.com hosts 250 million images of websites - not fully interactive like the WayBack Machine, but for many resources, still plenty. Another option: If a site is only down temporarily, a recent cache of the last time Google successfully crawled the page is often available via the page's Google search result (though if a site remains inaccessible, Google eventually drops it from the listings).
} 
temporary? Maybe. But [...] I don't think we really thought, 'What happens when the reader can't find this?' I know I've had some success when I go back with the WayBack Machine, at recovering pieces.

Neary's terms "stable-ish" and "vanish" both point to the distinction I am drawing between "stability" and "availability": Something can be available and move around. It can be available in a range of locations, from a range of providers. And external sources can provide availability - even if the text itself (to the extent a text can be considered a singular object on the web) is unstable. Thus, for Neary and her co-author Formo, putting their respondents' videos up on YouTube and linking to resources such as "Beyond the Red Ink" was essentially a no-brainer; it was the best possible way to connect with the wide audience they hoped to find via $C \& C O$.

James P. Purdy's (2009) research on Wikipedia editing ${ }^{49}$ pointed to some of the ways that the allegiance to stability has begun to fade in the Internet era. Purdy said:

Wikipedia (and wikis more generally) asks us to reexamine our expectations for the stability of research materials and who should participate in public knowledge making. [... It contests] the notion that online texts, even encyclopedic or scholarly texts, are ever permanently finished. This sense of public texts as infinitely changeable challenges conceptions of textual authority, particularly those associated with reputedly stable scholarly publications. (p. W352, W356)

Purdy described how changes to a page are all reflected in the Wikipedia history page; how conversations about changes are preserved on the "Talk" page- even though a page itself is a potentially infinitely changeable, including its title and URL (which Wikipedia calls "moving"), its history follows with it. Thus, the specific location associated with a

\footnotetext{
${ }^{49}$ Which was published, notably, in the 2008/2009 era of CCC, during which the print journal was publishing only excerpts of many pieces, with full essays available only via the NCTE website. Purdy's essay, in print, included just an abstract and the first two paragraphs of the article, ending with the notice, "Read the entire essay by James P. Purdy at www.ncte.org/cccc/ccc, 'The Extended CCC."' This effort was running simultaneously with the 2008 CCC Online (in which Collin Brooke and Derek Mueller wrote abstracts for all $C C C$ articles).
} 
page matters far less than the recorded social context that led to its creation and writing. That social context, Purdy emphasized, is deeply important to many Wikipedia editors; in the review he conducted, "over half of the changes were accompanied by a summary [on the history page] from an author-user" (p. W353). Salter's comments about a scholarly obsession with stability reflecting the "fleeting pursuit of location in scholarship" seemed to be making a similar point: As texts, academic and nonacademic alike, court revisions, feedback, and commentary from the public at large, where something can be found matters far less than that it can be found in one of the many automatic and/or archival social records of the Internet writ large.

Burton (2015) has suggested that accepting the ephemerality of online writing enables a renewed attention to the moment of reception: "When the pressure of longevity is removed, we are able to focus more intensely and critically on a text's reception in the current moment. When all we have is 'right now,' we free ourselves to dive deeper into the current rhetorical situation, to tailor our texts in real time." Focusing on a text's immediate reception shifts the key value from stability to availability and thus from "how can I make this last?" to "how can I make this accessible for the largest possible group of people?" Burton's comments call back to my earlier argument about the increased importance of kairos in online writing: As writing becomes more potentially ephemeral, our attention shifts from the way the text might speak to all of history to the way that it might speak to the current moment. Writing for reception in the immediate "now" relieves some of the pressures of academic argument—such ephemeral arguments are not meant to exist in perpetuity—and also enables writers to consider more fully the potential range of the immediate audience. By acknowledging that Internet texts are, to some 
extent, ephemeral, we open up space to write for the current moment, a space that has remained relatively unaddressed by academics in the humanities, occupied mostly by pundits and journalists - and thus we would make academic thought in the humanities available to audiences that have thus far been unable to access the academic conversation.

\section{Attention as Punishment and Reward}

The new allegiance to availability reflects an increasing sense among academic web authors that their texts can and should reach audiences that do not have access to the kinds of texts academics traditionally publish. Behind that sense is a hope for a wider audience - sometimes an audience outside the subdiscipline, outside the discipline, outside the university, or outside the author's lived experience entirely. To reach such audiences, academics must both attract and maintain public attention: I found much evidence in my interviews - and in other experiences I had while writing - that suggested academic web authors almost universally seek "outside" audiences of some variety, frequently succeed in attracting wider attention, and often engage in practices designed to keep what attention they have garnered. Yet, while attention can be a reward of online publishing (as many authors I interviewed described), opening a text to public attention also creates the potential for unwanted or negative attention; when authors release a text into the online wild, as any student of remix culture can tell you, they relinquish some control over its likely and possible uptakes.

That web authors conveyed a particular attention to attention would not surprise Richard Lanham (2006), who has described the Internet as the proving ground for the burgeoning "attention economy." Lanham argued that although many pundits have suggested that digital technologies have ushered in an "information economy," the true 
new commodity is not information (which is more plentiful now than ever) but attention (which is necessary for sorting through all that information). On the Internet, Lanham explained, readers experience constant

competition between word, image, and sound for our attention. [...] The Internet constitutes the pure case of an attention economy. 'Eyeballs' constitute the coin of the realm. If, as one sometimes reads, Internet companies spend 75 percent of their money on marketing, this only makes sense in a world where stuff has given way to fluff. It should not surprise us that the dominant discipline, the economics that matters in this new theater, is design. (pp. 17-20)

Michael Warner (2002) similarly described how, in the new media age, "Public discourse craves attention like a child. Texts clamor at us. Images solicit our gaze. Look here! Listen! Hey! In doing so they by no means render us passive. Quite the contrary. The modern system of publics creates a demanding social phenomenology" (p. 419) - the social phenomenology that Lanham termed the "attention economy." Of course, the attention economy necessitates that corporate websites compose to maximize "eyeballs"; to be profitable, a website must continually attract new readers and keep the attention of the ones it gets. A commercial website's success is measured in its ability to optimize for search engines, in its daily page views and bounce rate, in the number of "impressions" it can sell to advertisers. But even in academic web text, web authors seem to have internalized this understanding of the attention economy: They engage in practices that demonstrate a desire to attract attention beyond the normal bounds of the academy, and a desire to maintain the attention they have attained.

We have recently paid significant disciplinary attention to virality, the way ideas and images get picked up and spread rapidly across the Internet—see, for instance, Laurie Gries's (2015) analysis of the Obama Hope image and Ben Wetherbee's (2015) 
reconceptualization of topoi as memes. The rise of virality has the potential to make public attention seem fickle, but scholarship such as Gries's and Wetherbee's suggests that rhetoricians are uniquely poised to understand the conditions that contribute to the spread of viral communication. Further, because we study discourse communities, we are poised to understand how to suss out the unarticulated rules of a writing communityand thus to pen a message that comes across as genuine and relevant, not manipulative and gimmicky. Public attention, as the writers I interviewed reiterated, is reactionary and social and fleeting; yet it also can be courted to some measure of success, even by academic authors who are not especially experienced in writing text for the web.

Several authors suggested that the notion of public attention for academic work seemed almost oxymoronic. In fact, the fifth definition of "academic" in the Oxford English Dictionary is "of no consequence, irrelevant." Michael Warner (2002) argued that "the fate of academic publics" is to almost never attract public attention or have any kind of political relevance — even "when academics claim by intention or proclamation to be doing politics" (p. 421). This relegation to political irrelevance is, Warner said, because academic discourse is not "punctual" or "abbreviated" but instead has "longer rhythms or more continuous flows" (p. 421). Yet publishing online offers a different experience, John Duffy explained:

When you're an academic, you're used to living and dying on every word you write and putting so much time and sweat and care and then having nobody pay any attention. At least that's been my experience. I've written a lot of different things. But these pieces ['Virtuous Arguments,' IHE'; and 'The Ethics of Metaphor,' the Chronicle] seem to have touched a nerve. 'Virtuous Arguments,' particularly, I was really surprised by how many people have either approached me personally to talk about it, or wrote to me. There was a pretty [good] backand-forth on Inside Higher Ed. I've been surprised. It tells me that these questions matter. And that we are not nearly as far enough along as we can be in discussing how to address some of the problems of our public discourse. 
In explaining how "Virtuous Arguments" "touched a nerve," Duffy referred to the comment section on Inside Higher Ed - which, at nearly five years after its publication, holds at just under 50 comments. One anonymous commenter took issue with Duffy's examples, suggesting the piece had a leftist bias that undercut its argument. Donald Lazere called for attention to virtuous argument in CCCC and the National Task Force on Civic Learning and Democratic Engagement. But perhaps most interestingly, several comments from communication studies scholars - including one signed by Trevor ParryGiles, the Associate Director for Academic \& Professional Affairs of the National Communication Association-reminded Duffy that similar kinds of important work are going on in other places in the university, namely communication departments. The speed at which this happened is worth highlighting: All comments I've described above had appeared by 5:30 pm on the day of publication. What academic publication could generate public political discussion, inspire intradisciplinary calls for change, and provoke interdisciplinary calls for collaboration within the space of a working day (a Friday, no less)? What Warner calls the "longer rhythms" of academic discourse would normally have stretched out this kind of exchange over months (at least) or (more likely) years. Duffy suggested that his online writings attracted more attention within the academy than any of his previous, more-academic writing - the Dean called him, after "Virtuous Arguments" came out, to congratulate him on the piece, and he was later asked to lead some university-wide workshops on ethical argument. Anastasia Salter said similarly that although she has written plenty of more traditional academic texts, her lessscholarly blog writing with the Chronicle has really made her name: "Most of the 
invitations I get to speak," she said, "are because people have read something on ProfHacker and want a workshop on that type of topic."

\section{When Getting Noticed Goes Wrong}

Attention is, of course, not universally positive. And given the editorial concern about instability of web resources, online academic authors can sometimes be surprised by the persistence of online texts that were intended to be temporary. That is, online texts often do not disappear in the way writers want or expect them to. Reminders of this Internet truth seem to have been following me around as I have worked on this dissertation: When I arrived at University of Illinois Urbana-Champaign (UIUC) to interview Kelly Ritter for the previous chapter in May 2015, the university was embroiled in controversy following remarks Steven Salaita, an English professor at Virginia Tech and a 2014 new hire in the UIUC American Indian Studies Program, had made on Twitter about the 2014 Israel-Gaza conflict. Salaita's tweets had argued, in strong language, that Israel's bombing was a genocide committed by a colonial power against indigenous Palestinians; he also, somewhat ironically, critiqued the overapplication of the term "antisemitic": "If it's 'antisemitic' to deplore colonization, land theft, and child murder," one tweet asked, "then what choice does any person of conscience have?" To emphasize the potential permanence and rhetorical circulation of Internet texts, I want to note that I am able to cite these tweets and report them in social context because a New York Times article (Mackey, 2014) embedded a nine-tweet selection of them. The UIUC chancellor received many complaints about the tweets, accusing Salaita of anti-Semitism, and the board voted soon after to deny his hire. Apparently, it was not sufficient that the director of the UIUC Initiative in Holocaust, Genocide and Memory Studies defended Salaita. 
Apparently, it also was not sufficient that Salaita's comments were influenced both by his experiences as a Palestinian-American and by his scholarship - which included, for example, an essay (2005) in College Literature called "Ethnic identity and imperative patriotism: Arab Americans before and after 9/11." Indeed, had Salaita been hired, it seems likely that he would have published soon in an academic journal on the same controversy, likely making the same argument (though surely without an off-color joke about Netanyahu wearing "a necklace made from the teeth of Palestinian children"). His positions on colonization and his support of Palestine could hardly have been news to the committee that offered him the job. His primary error appeared to be making that argument in a place where it attracted more attention than any English literature academic journal ever could.

This was the example weighing on Ritter's mind when I asked her whether College English might go open-access online. Given the current subscription-based funding structure of the journal, she said a full open-access model was impossible. Yet, upon taking over editorship of the journal, she'd made two open-access-oriented changes: Now, every College English issue has one open-access piece. Nodding to Salaita and Twitter, Ritter added that she also started a College English Facebook page—it seemed, she explained, a "relatively inoffensive" platform, compared to the amplifying nature of Twitter. The College English Facebook page did indeed seem to drum up more excitement for releases; Ritter cited a September announcement of a new issue that had received over a thousand views in the first 24 hours after she hit "post."

When Ritter called Facebook "relatively inoffensive," I found myself inclined to agree. After all, Twitter is an intentionally public forum, and Salaita had tagged his posts 
with high-volume hashtags such as "\#Israel," “\#Gaza," and "\#FreePalestine." The visibility of Facebook posts is much more limited, usually available just to the poster's selected "friends." 50 That was before my former colleague Joy Karega, Oberlin College rhetoric and composition professor and University of Louisville alumna, found herself in Salaita-flavored hot water, accused of anti-Semitism following a series of 2015 Facebook posts (Karega was, at the time, posting to Facebook with "public" visibility). Like Salaita, in the offending posts, she explicitly decried the overuse of the term "antiSemitic" to dismiss legitimate critiques of Israeli policies — and one post, significantly, ended with the call for "more of us being willing to venture into these areas," to "speak up and stop being silent or afraid to speak on these things" (Gerstman, 2016). Karega's key rhetorical overstep, apparently, was suggesting that "Rothschild-led bankers" and the systems of power they represent, might have helped orchestrate several terror attacks. ${ }^{51}$ In Karega's case, however, the university came out in public support of her right to free speech.

Though being shoved so roughly into the public spotlight must have been uncomfortable for Karega, I note that the experience granted her an opportunity rarely granted to rhetoric and composition professors: With her public statement, she got the last word in a New York Times article about the controversy (Rogers, 2016). "It is truly regrettable," the statement began, "that an institution such as Oberlin College, with a historical legacy of activism and social justice, particularly in terms of African

\footnotetext{
${ }^{50}$ Of course, most Facebook users have at least a story or two of a time when the response to a post reminded them just how much wider that "friendly" public is than it seems at first. Mine are thankfully relatively innocuous, e.g. Meeting a friend of a friend who says, "Oh yeah, I recognize you from Facebook."

${ }^{51}$ I am far less convinced than Karega, for what it is worth, of such things as CIA involvement in ISIS and Israeli involvement in $9 / 11$.
} 
Americans, is being used as a personal tool of religious extremism by a small number of people" (Rogers, 2016). Further, now assured of security in her position at Oberlin, Karega has already begun to turn the experience into research. Forward, a JewishAmerican periodical, actually quoted her research agenda at length, which included questions such as, "How has the uncomplicated tying of conspiracy theories to accusations of anti-Semitism been used (is being used) to control the parameters of inquiry and research in the academy?" The author of the post then rather erroneously summarized, "In other words, Karega wants to study how Jews silence anti-Semites"which, to me, simply serves to make Karega's point. Regardless, I would be hard pressed to name a rhetoric and composition scholar who has had a bigger influence on national conversations in 2016: Karega sparked national discussion about free speech, about academic freedom, about the rhetoric of conspiracy theories, and about the silencing use of the term "anti-Semite." That is some impressive critical, rhetorical work for a handful of Facebook posts.

\section{Short Form Writing and Attention-Keeping Hyperlink Practices}

Academic rhetoricians have tended to fare pretty poorly in this attention economy, especially in spaces where we are self-consciously taking advantage of digital affordances: I believe this may be attributable to the fact that many of our digital pedagogies and digital analytics are drawn from the New London Group's (1996) pedagogy of multiliteracies and other derivative theories of new media. We study and create digital objects that are multiply mediated, that are "redesigned," that represent a wide range of design elements, that enable us to give flesh to a metalanguage of multimodal design. Coming from a tradition of electronic literature, we get excited about 
digital work that seems to push the boundaries of what text can or should be. Cheryl Ball (2006), for example, specifically named the NLG in her reasoning for selecting a particular video for analysis (in a piece that argues for a "readerly" rather than "designerly" rubric for analyzing multimodal texts in writing studies): "I use a FLASHbased text called 'While Chopping Red Peppers' (Ankerson and Sapnar, 2000) because it is a multimodal text that combines all five of the NLG's basic modes of meaninglinguistic, audio, spatial, gestural, and visual." Though other authors engaging in multimodal analytics may not be as explicit about the NLG-inspired justification for their text selection, I suspect that a similar reasoning lies behind many if not most of the digital texts that end up discussed in writing studies journals. To perhaps belabor the obvious: Genres that excite those of us fascinated by experimental hypertext poetics - genres that engage many tenets of new media theory—are likely not the same kinds of genres that capture the attention of the wider Internet public.

Indeed, there has been remarkably scant writing studies attention, Pamela Takayoshi (2015) pointed out in a recent Computers and Composition article, to the "short-form writing" that is "increasingly prevalent across the culture." Henry Jenkins's $(2004,2006,2012)$ work on participatory culture addresses a range of online short-form fan texts; Bronwyn Williams's (2006, 2008a, 2008b, 2009, 2012 with Amy Zenger, et al.) work on popular culture and social media literacies considers mostly short-form online multiliterate writing of students and other young people in an increasingly networked society. Yet considering how much attention writing studies researchers have paid to multimodal texts, Takayoshi (2015) argued, the questions that still remain unaddressed about this kind of short-form, networked writing are surprisingly basic and enormous: 
[C]omposing processes, too, are changing in the production of short-form, interactive, socially-networked writing, and writing studies does not yet have a clear sense of what these technologies demand for writers at the level of the composing process. What are the rhetorical demands of short-form writing? What are its features? What are its processes of making meaning? Are short-form and elaborated forms of writing equivalent in terms of the rhetorical and composing demands on writers? (p. 10)

Takayoshi's study of the composing processes of people on Facebook seems indeed a meaningful initial foray into addressing these kinds of questions. But I believe we remain, in the subfield of computers and writing more broadly, overly focused on genres that seem to evidence "interesting" design, leaving the vast expanses of short-form networked Internet text undertheorized, despite its now decades-long hold on the web's attention economy.

The authors I interviewed, even those new to online writing, evidenced a deep sense of the competition for attention when they described their hyperlinking practices. Several authors described choosing which hyperlinks to use, how many hyperlinks to use, and where to place those hyperlinks via a careful marshalling of reader attention. Daniel Schafer put it succinctly: "I think we were probably conscious of the fact that we wanted to encourage people to consume the infographic at the very least in chunks, so we were careful not to provide too many opportunities to navigate away from it." Dawn Formo similarly expressed concern that "filling [the piece] up with a bunch of hyperlinks" might distract from their message, "burying a comment we are trying to make by just directing people all over the Internet." Though some writers have expressed concern about the way that parenthetical citations damage the readability of a document (e.g. Connors, 1999), there seems to be less concern about the way a citation might encourage a reader to put down the piece at hand and instead seek out a cited source. This is, of course, a 
possibility: I have encountered a parenthetical citation to another piece that looked interesting and gone off in search of it, putting down the citing text for the time being.

Yet the at-handedness of a hyperlink citation seems to make the possibility of losing a reader's attention more salient in authors' minds, and they link with more caution, with deeper awareness of the importance of maintaining attention. Cate Blouke described hyperlinking, thus, as "more useful" than other kinds of citation practices, particularly parenthetical citations:

Parenthetical citations, I tend to ignore unless I am particularly interested in the thing that is being quoted or talked about, and then I'll go-I prefer, I wrote my dissertation in Chicago style, because I like footnotes. I like to be able to look down at the bottom of the page and get at least some sense of what I'm looking at. But if it's all the way back of whatever the thing is, I'm much less likely to go track it down, unless it seems particularly relevant to my interests. Whereas with hyperlinking, it's so immediate, that I'm like, 'Huh, that's interesting!' And then I can click on it.

With a hyperlink, there is decreased friction in the experience of seeking out the linked source. A hyperlink commands attention, in its materiality; its underline or changed color makes it stand out, quite literally, from the rest of the text. It proposes an attention shift"click on me to know more." And thus Internet writers, aware of the purity of the Internet's attention economics, link with caution. They hold back their hyperlinks for fear of losing interested readers.

\section{Spend Attention to Gain Attention}

Ultimately, my attention to attention has a pragmatic bent. I return, in my mullings, to a series of "how" questions: How can rhetoric and composition scholars manipulate the attention economy without spending money? How can we make better use of the affordances of short-form networked Internet text? How can we do all this without 
a huge marketing budget? How can we do it without betraying the new allegiances of online writing and the older-yet-still-salient allegiances of academia (such as intellectually balanced interrogation, awareness of metadiscourse, factual correctness, etc.)? Though I did not, in this series of interviews, broach these "how" questions, my preliminary answers seem tied to this new allegiance to attention. We can gain attention without spending money, that is, by being willing to spend attention—which Lanham called the "coin of the realm" - on these kinds of short-form networked texts. Attention is certainly in somewhat short supply for academics, so "spend attention to gain attention" is not an incredibly easy sell. But given that achieving a wider Internet audience can support our scholarship — and I think it can, both in the short and long term — then there is a significant potential disciplinary, cultural, and eventually perhaps even financial gain from it. We spend attention to gain attention; gain attention to garner disciplinary support within and outside the academy; leverage that attention to have a foothold in the national conversations where we think our work is important—such as, in the call for more-ethical public discourse, or in the call for attention to silencing uses of the "anti-Semitic" epithet. The unstated premise of this enthymeme: We must be willing to share our work, early and often, with a wide and intentionally global audience.

\section{Sharing}

The allegiance to attention depends on comorbidity of another allegiance, to sharing: There is, I am far from the first to argue, a significant shift in culture toward a value of sharing: Pointing to the rise of the home-sharing services AirBnB and CouchSurfing, car-sharing services Zipcar and Lyft, digital sharing services such as Flickr and YouTube, and notions such as "coworking" spaces where freelancers park 
themselves to experience the feel of an office, Rachel Botsman and Roo Rogers (2010) described this trend as "collaborative consumption." Botsman and Rogers attributed this cultural shift to "[t]he convergence of social networks, a renewed belief in the importance of community, pressing environmental concerns, and cost consciousness" (p. xx). Social networks indeed encourage increased sharing, from pictures of food to home remedies to political arguments. Journalist and professor Jeff Jarvis (2011), arguing for the value of sharing in private and professional life, reported an interview with Facebook founder Mark Zuckerberg: “Zuckerberg has his own, social version of Moore's law [which predicted in 1965 that the potential number of transistors per square inch of a circuit board would double annually] —I call it Zuck's law, though he doesn't. It decrees: This year, people will share twice as much information as they did last year, and next year, they will share twice as much again" (p. 23). Governments are dragged (screaming, kicking) into compliance with the Internet allegiance to sharing, as organizations such as WikiLeaks make millions of government documents available to the public eye and as citizens come to expect politicians to make their personal (financial, health) and political (speech transcripts, emails) records publicly available online. The "free culture" movement (Lessig, 2004) values sharing of creative work; political "pirate parties" around the world call for the abolition of copyright restrictions for non-commercial purposes - in Sept. 2016, Pirate Parties International named 69 countries with pirate parties; in 2015, the Icelandic Pirate Party became the largest political party in the nation ("The Pirate Party"). Education, too, has become increasingly shared widely online, often available free. Armed with resources from iTunes University, Coursera, Codecademy, KhanAcademy, and university-sponsored Massively Open Online Courses, the motivated 
autodidact can spend all day in structured learning experiences for nothing more than cost of a computer and an Internet connection. Project Gutenberg has a lifetime of books to read. Instructables and WikiHow seek to fill the gap left by disappearing shop and home economics classes. $^{52}$

To create a public, Michael Warner (2002) has said, sharing is the first step. The word publication itself seems to suggest an allegiance toward wide distribution-it is, of course, the making public of a text. Yet Warner argued that a public only forms when people begin to pay attention to a publication (p. 419); when we publish, Warner said, a public coalesces around a text, a public of exposed and potentially interested people, who may or may not take up that text and engage in action as a result of having seen it. However, the history of academic publication has not maintained that allegiance to sharing, in the broad sense: Much of our work is (and has long been) published in scholarly journals, which were long available only in university libraries and now are often available online only behind a paywall that makes accessing academic texts expensive if not wholly out of reach for people outside of the university walls - and inside university walls in poor countries. In an international New England Journal of Medicine study, Barbara Aronson (2004) found that 56\% of institutions in the lowest income tier had absolutely no subscriptions to international journals. There have been significant inroads into open-access online publication; one study suggests that [40\%] of all academic work is now published open-access, freely accessible online. Some of the

\footnotetext{
${ }^{52}$ But perhaps it is not quite correct to describe education as "free"; rather, it is freemium - you can access knowledge for free, but people continue to pay for formal education because a diploma still has cultural significance, and because many of us remain believers in the magic of face-to-face pedagogy and a classroom full of learning colleagues.
} 
print-primary scholars with whom I have shared this project have suggested that the notion of free, open-access academic publication is naive. Kelly Ritter pointed out (quite reasonably, of course) that academic journals have significant operating costs, from copyediting to maintaining a website to printing and distribution. For such journals, full open-access would eliminate the potential of funding, which they rely on to keep their presses going. Even Doug Eyman and Cheryl Ball pointed out that the funding model of open-access publishing is unsustainable without unpaid labor: Kairos's editors are all unpaid.

Yet authors expressed to me, over and over again, the desire to share their workand all of their work, often even early-stage work like conference presentations and coded datasets - with the broadest possible audience. Paul Muhlhauser (who is a Harlot editor), Cate Blouke, and Daniel Schafer (2016), for instance, coauthored a Computers and Composition Online text arguing that academic work should be designed to appeal to wide audiences, by presenting texts at the appropriate kairotic moment and making text available in several different levels of complexity simultaneously. Their $C \& C O$ webtext, "May the \#Kairos Be with You: Accessibility, Authdi, Veils, and Star Wars," demonstrated this principle in both form and content. The piece is presented in three different formats: An infographic, a $C C C$-style long-form text, and a "toggle" that provides links from one to the other, section by section. Muhlhauser, Blouke, and Schafer seized the kairotic moment of the release of the new Star Wars trilogy by theming their piece around Jedi and the Force - the Force is like kairos, and authors are "Rheti." "By composing in multiple formats," they explained, "a Rheti is being more cooperative with readers/audiences rather than being directive or prescriptive." 
Thus, they argued, accessibility ought to go beyond seeking to make work available to audiences with physical disabilities; truly accessible academic work would consider audiences with a wide variety of literacies and seek to meet readers with a wide variety of prior experiences, interests, and even time constraints. Nodding to an allegiance to availability, Schafer summarized:

These ideas are things that we don't necessarily want to be relegated to a genre that some people consider to be less accessible, either because you physically can't get access to some articles, or because you don't have the relevant training or background or whatever to just jump into an article like that, or because you don't have the time to do it.

Presenting ideas with sensitivity to an audience's potential range of interests and backgrounds has a wide range of positive implications: It broadens the potential audience of a publication, including to readers who are not (yet) academics. It may encourage undergraduates to read earlier and more widely in their chosen fields, thus improving professionalization and knowledge mobilization. It improves the rhetorical velocity of a publication, making it easier for people — academic and non-academic alike - to pick it up for different purposes.

All three "May the \#Kairos" co-authors suggested that this value of making their work accessible to wide audiences, beyond those that are traditionally academic, drives their work more generally. Schafer, for example, discussed how when he and Muhlhauser have collaborated on conference presentations, they share those presentations online:

Our conference presentations that we've done, I usually feel like we've made our presentations public also, and we kind of design our conference presentations in a way that they can be shared and consumed even if we're not there to babble about what it is that we're trying to show the audience. 
Blouke, in addition to putting versions of her conference presentations online, has worked for a couple of websites that publish short-form analytical posts, responding to images from popular culture, free and available to the public: Viz, the Digital Writing and Research Lab publication out of University of Texas at Austin; and BAGNewsNotes, now ReadingThePictures.

For Muhlhauser, the allegiance to availability and accessibility goes beyond the text more broadly and extends to decisions he makes about his citation practices. Muhlhauser, who designed all the webtexts he coauthored with Schafer and Blouke, has developed the consistent—-but rather disciplinarily rare — practice of linking to all of the tools he uses in creating the texts; for instance, if he consulted a CSS tutorial in designing the page, he will name the creator of the tutorial and link out. "May the \#Kairos" also linked to a number of interesting browser plug-ins, tangentially related to the text, that demonstrate the commercial undergirdings of the Internet. When I asked Mulhauser about one of these tools in particular, he emphasized the importance of sharing:

I just wanted to share it because I'd learned about it from a conference. [...] It just seemed very exciting for rhetoric, just something I wanted other people to see so maybe they could write about it. That's kind of the thought process of that, giving people access to the tools, showing them that they exist. [...] 'Oh, this is how you do the 3D scrolling. I want to click on that, figure out how to do that,' and click it. Like, the readymade for CSS animation, 'I'm going to click on that, because it'll take me to what I want to do.'

This ethos of sharing everything from the source materials to the tools used to create a webtext seems to run counter to older-style academic attitudes of protecting jealously guarded secrets - and it represents a move toward not just recording a history of the author's research but also providing new directions and opening up new opportunities for future researchers. 
Though Muhlhauser is in favor of sharing resources, attributing those resources to their creators is also hugely important to him; he described the process of linking as "giving people honor," citing Clay Shirky's (2011) notion of "cognitive surplus," which described how creative users of new media can put the tools to communal and civic uses. "Cognitive surplus" suggests that there's latent societal value in the amount of attention being spent on sometimes creative, sometimes frivolous, sometimes intentionally productive Internet activities, and it is up to thought leaders to put that surplus to good use - to look at the amount of time people spend playing dumb pattern-matching games and realize, as the McGill Centre for Bioinformatics did with the game Phylo, that those same people might want to play a game that helps sequence the human genome.

The latent potential of cognitive surplus is the ideal, maybe idealized endpoint of the value of sharing; people often will share with only a vague notion of the public that they are creating in publishing. Yet for that potential to be realized, people must share regularly, before they can imagine how things might be taken up. When I interviewed Cheryl Ball, she was enthused about a project she and some collaborators (including Karl Stolley, Collin Brooke, and Derek Mueller, among others) had recently started—what she terms a "boutique data repository" for writing studies. The theory behind it: We do lots of research, but for the most part we have not previously shared our raw data with one another. That means that studies may duplicate one another, that people may waste time collecting data when, actually, there are already extant datasets that meet the need - a key part of Richard Haswell's (2005) call for replicable, aggregable, data-driven research in writing studies. With this call in mind, Ball et al. started rhetoric.io, a place for writing studies researchers to share their raw data. Like Shirky's notion of "cognitive surplus," 
this sharing is built on the idea that someday, somehow, some people may be able to make use of this stuff again. Boutique data seems like the ultimate anti-foundationalist product, maybe the purest form of what Stanley Fish (1989) terms "theory hope," sharing the burden of contextualization and situations and "knowing how" with the rest of the field, maybe even the rest of the world. We cannot know how or why or when data will be used again, now—but if we do not share it, then surely it will never be used again. Rhetoric.io opens up the possibilities that future writing studies projects may be longitudinal, may be metaanalytic in a way that has not yet been possible. ${ }^{53}$

When we open our research to a potentially non-academic public, yet more possibilities emerge. John Duffy has pointed to some such possibilities, even attributing the direction of his research in recent years to communication with the "general public":

I gave a talk at a local university up here, about four or five years ago, and it was to the general public. [...] In this particular case, I had run through all the kind of offenses to public discourse that I see on a daily basis. I was making the case that writing programs are addressing this and that writing programs have the potential to shape the future, and I was giving numbers and so forth. In the Q\&A, someone said, 'If writing programs are doing such a great job, how come the discourse is so lousy?' I thought, 'That's a great question.' That's kind of... in a way, it kind of influenced a lot of what I've done since, because I've been trying in the different pieces I've been writing, to talk to our field and say there's something that we could and should be doing.

"Virtuous Arguments," in fact, came almost directly out of this experience; Duffy came to believe that it was imperative he send the public question back to other writing program administrators: What can we be doing better to improve the quality of public discourse? My answer to that question is a bit of a departure from Duffy's; while I

\footnotetext{
${ }^{53}$ To take the notion of "cognitive surplus" to its fullest extent, it opens potential for interdisciplinary collaboration: For now, all the datasets on rhetoric.io are from and apparently for rhetoric researchers. But should it take off, it could allow for engagement from outside the discipline.
} 
sympathize with many of the concerns Duffy raises about the importance of teaching ethics in first-year composition classes, I am far more frustrated with the way that the writing research we are doing seems to be systematically ignored—even by, say, firstyear composition instructors who come from the literature tradition instead of the rhetoric and composition tradition. ${ }^{54}$ That is, we are doing lots to improve the quality of classroom discourse, of discourse that comes out of our classrooms, of the discourse of students who come out of our classrooms. But we are doing a lousy job- to borrow Duffy's critic's term—of making that work visible beyond the composition classroom. And thus, writing studies journals become a strange kind of echo chamber, where we are studying culture and communication without often giving back to the larger culture or to larger communication practices. Rhetoric divorced from the public is a strange, insular beast indeed.

\section{What Does "For the Public" Even Mean?}

My proposal: We are not connecting with the public because we are not creating a body of accessible short-form Internet text that is made to be shared broadly and widely, connected to relevant and timely issues. Such a body of work would enable the potential of drawing wider-spread public attention, and as Warner (2002) pointed out, attention is absolutely necessary for the creation of a public:

A public is constituted through mere attention [...] Because a public exists only by virtue of address, it must predicate some degree of attention, however notional, of its members. The cognitive quality of that attention is less important than the mere fact of active uptake. Attention is the principal sorting category by which members and nonmembers are discriminated. (p. 419)

\footnotetext{
${ }^{54}$ See, for example, Joseph Teller's October 3, 2016, essay in the Chronicle, “Are we teaching composition all wrong?" Teller put forth a strawman of an argument: That composition courses aren't teaching students to make rhetorical decisions.
} 
We must not simply address the public (which we do in venues such as Harlot, and at least superficially in venues such as Kairos and $C \& C O$ ); we must seek active uptake, with practices designed to seek and maintain attention. Thus, when I speak of reaching "the public," I mean a public that is hailed, by a publication with rhetorical velocity, to pay attention. "The public" is a handle for the notion of an audience "beyond""general public" to the extent that such a thing is possible. Warner (2002) has argued that the very idea that we could address a "general public" that includes everyone "seems to have something wishful about it":

To address a public we don't go around saying the same thing to all these people. We say it in a venue of indefinite address, and hope that people will find themselves in it. The difference can be a source of frustration, but it is also a direct implication of the self-organization of the public as a body of strangers united through the circulation of their discourse, without which public address would have none of its special importance to modernity. (p. 418)

That is, though when we speak in a "venue of indefinite address," we are not truly speaking to the entirety of the "general public," whatever that means; rather, a diverse subset of "everyone" will, hopefully, encounter our message, willingly engage with it, and perhaps make cognitive or purposeful use of it. In so doing, they become the "public" we address. Over time, Warner claimed, publics organize themselves by being addressed by the same sorts of texts. Warner's notion of a self-organizing, text-oriented "general public" is what I wish to engage in suggesting that we ought to develop a short-form web publication for the public (a proposal I will flesh out in the conclusion to this dissertation). Jarvis (2011) described attention as "the obvious advantage of going public" (p. 38). We need to engage in practices that encourage and allow for the 
possibility of an audience wider than we usually attract; such practices will allow us to shape a public around the web texts we create.

\section{Linking to the Public: Directions and Implications}

When I began research for this chapter, I expected to find authors discussing guidelines about how to hyperlink: Don't link in X situation. Do link in Y situation. Don't make your anchor text longer than $X$. I even had questions that were slightly leading

along those lines_- "Has anyone ever given you explicit guidelines, such as..." and then I offered a big long list of example questions. Yet none of my authors said yes to that question; all said no. Instead, authors emphasized that they were always creating their own best practices. Linking thus became, instead of the topic of the chapter, a specific textual practice that gave me a way to focalize discussion of more general attitudes and more general textual practices, within a very specific discursive context—one that asked writers in each instance to situate themselves in terms of the other text they are citing, in terms of the other work they have done, in terms of models they were drawing upon, and so on.

All of the authors I interviewed also write traditional print academic text, so their sense of hyperlink citation practices was - and my questions likely primed them to be this way-a little binaristic: How is writing for a webtext different from writing for print? Have you ever written for popular press online work, and if so, how does it affect your understanding of online academic writing? How are hyperlinks different from print citations? What comes into play in your decisions about how/when to cite that doesn't come into play in your decisions about how/when to hyperlink, and vice versa? Thus, I suspect they felt compelled to maintain the binary. Obviously I recognize - and many 
authors pointed out—-that there are many similarities between print and hyperlink citation; and, conversely, hyperlinks serve some purposes that parenthetical citations cannot (and vice versa). I believe it to be a productive fiction nonetheless, because it enables writers to draw out a felt sense of a tectonic shift.

Another way, though, to understand this shift, is to consider how writing from the not-too-distant past that characterized Internet writing no longer really applies to Internet writing today. Warner's (2002) "Publics and Counterpublics" explicitly considered the potential of a public to organize around a series of Internet texts - and was dismissive of the notion:

At the time of this writing, web discourse has very little of the citational field that would allow us to speak of it as discourse unfolding through time. Once a website is up, it can be hard to tell how recently it was posted or revised, or how long it will continue to be posted. Most sites are not archived. For the most part they are not centrally indexed. The reflexive apparatus of web discourse consists mostly of hypertext links and search engines, and these are not punctual. So although there are exceptions, including the migration of some print serials to electronic format and the successful use of the web by some social movements, it remains unclear to what extent the changing technology will be assimilable to the temporal framework of public discourse. (p. 421)

Many of the affordances and allegiances of contemporary webtext discount Warner's complaints: The practice of "link chaining" I described in my first chapter seems to be precisely the sort of "discourse unfolding through time" that Warner proposed; authors quite commonly now begin short-form networked text with a paragraph that briefly glosses the web conversation on the issue to date, with a collection of relevant hyperlinks. Most sites now structure posts on the site into reverse-chronological feeds, each post with publishing and update timestamps. Many sites, Wikipedia a pioneer among hundreds, actually allow readers to go "back in time" and access previous versions of a page, 
considering its many revisions in minute detail. The increasing popularity, reach, and resources of web analytics services such as Alexa and web archive services such as the Wayback Machine indicate a much more highly developed "reflexive apparatus of web discourse"- not to mention the hugely developed social apparatus of Facebook, Twitter, and the like, which provide annotated, running social commentaries on web content.

In a moment of evolving guidelines, when practices are still wholly tacit, as the genre of short-form networked web opinion writing becomes more stable-for-now, there is a particular opportunity to see how values shape practices, to understand the thinking that goes behind the development of practices. For academics in rhetoric and composition who seek to write for the web, this is a moment of great potential: By analyzing these allegiances, we can develop practices that reflect those values. I am reluctant to describe any practices as "best practices"- because the term has an inappropriate note of finality, seems to deny the potential for shifting contexts, and attempts to turn idiosyncratic judgment into universal rules. Nevertheless, I believe that all ideal web writing practices would take advantage of the affordances of the web and reflect the web's new allegiances, to what extent possible. A publication that is not developed with the new allegiances of web writing in mind runs the risk that the writing will seem tone-deaf.

A well-aligned text will take advantage of the web's major textual affordances: For instance, its ability to be timely, responding directly to a current situation, connected to a quickly glossed conversation among other similar texts (according to the allegiance to kairotic exigencies); its ability to reach a potentially global audience, including readers of ages and stages and education levels and cultural backgrounds dramatically outside the audience originally imagined by the author (according to the allegiance to availability); 
its ability to possibly be circulated rapidly or even virally without increased distribution costs for such a wide audience, with practices that encourage that wide circulation (according to the allegiance to sharing) and maintain the attention of the audience gained (according to the allegiance to attention). Taking advantage of these affordances, however, means ceding some control over the range of uptakes a text might have.

The notion of accepting a loss of control might be another allegiance of online writing. Salter gestured at the idea when I asked why she had decided, at one moment in "Alice in Dataland," to link to a Tumblr tag for the phrase "pool of tears":

In a sense, I've given up control. I knew what the tag was the moment I linked it. And I know what the tag tends to attract. If "pool of tears" suddenly becomes the new hot word for pornography, then I suppose I've taken a risk. And there is a risk in going to a live tag. And it's also a part of the work in a sense, like a connection to civilized discourse. To ongoing discourse, which I think can be very interesting and we don't always take enough advantage of. The work lives on the web and connects to an a constant conversation.

Salaita's and Karega's experiences in connecting "to ongoing discourse" similarly point to both its risks and rewards; yet, as Salter makes clear, even behavior that is less intentionally provocative can lead to a loss of control of the text and its uptakes. I do not mean to suggest that authors of print texts have anywhere near total control over the possible uptakes of their work; that would clearly be specious. Yet I do argue for a significant difference in the degree of unpredictable uptakes for texts that are published for a wide audience online, compared to texts that are published for much more smaller audience (smaller by number, smaller by geographical reach, smaller by disciplinary reach, etc.). When a text is presented in a publication with high rhetorical velocity, its uptakes are less constrained to similar kinds of rhetorical situations. That is, an academic text published in an academic journal will likely be cited by other academic texts in the 
same or similar kinds of academic journals - and likely not elsewhere. A text published by an academic on the Chronicle will likely reach readers outside the author's discipline, almost surely some readers outside the academy. A text published somewhere yet-more intentionally public, such as Slate, will surely reach readers outside the country; translated into other languages, it gains yet more rhetorical velocity. Likely and possible uptakes each occur on a range; the range of both is wider, however, for widely available, rhetorically velocitous Internet publications than for any strictly academic publication.

In future research, I would like to broach the notion of new allegiances of online writings more explicitly with authors and editors, with the goal of seeking out yet more new allegiances and beginning to understand, in more detail, the way that they affect the writing and reception of Internet texts. I am not convinced that this method of discoursebased interviews about an individual discursive element is absolutely the best way to encourage authors to discuss the new allegiances of online writing; it certainly did help me get at some issues that are tacit, but it would be nice to pair that investigation with a follow-up interviews, asking authors to explicitly name some things they think are different about how we use online writing, what social functions online writing serves, and so on. In addition to picking a single discursive element, for example, I could ask writers to bring in one online text (academic or not) they have written and one print text (academic or not), and ask them to compare the experiences of writing — rhetorical situation, things they considered, questions they had while writing, etc. Asking flat-out, instead of analyzing the protocol, of course, would make me doubtless get a different kind of reflection, shaped by a desire toward "fidelity." Such methodological triangulation would be helpful and interesting. 
Furthermore, given that my argument is that these are emerging practices, developing at a time when allegiances are shifting, I would appreciate a more longitudinal perspective. Will authors develop more internally and broadly consistent hyperlink practices over time? Will authors' senses of the distinctions among web genres change? I noted how often authors seemed insecure about answering the questions I asked - asking them about their hyperlink practices seemed to put some of them on the defensive. I heard people say things like, "Oh no, what did she notice?" This response was so common, in fact, that as I conducted more interviews, I actually changed my interview protocol to include a brief blurb at the head of the discourse-based portion that assured authors I was asking for the sake of curiosity, not to call anyone out for "inappropriate" linking. I believe the greatest benefit of focalizing discussion on an individual practice is that it seemed to keep interviewees from feeling like they had to be a "general expert" and instead were simply being asked for a picture of their mind, a sense of what was going on in their heads as they wrote. Yet most suggested it was the first time they had ever been asked to justify or explain their hyperlink practices. I am curious now, then, to see if any of their practices have changed as a result of having being part of this research, being forced to become more aware of the

Ultimately, the study in this chapter points to the need for more disciplinary attention to short-form networked Internet text, particularly web opinion writing, which has been seriously undertheorized despite its increasing hold on the attention of the social media public. It also suggests a series of practical guidelines for a web publication in rhetoric and composition that is aimed at that social media public: Such a publication should, as a rule, seek to connect its texts to for recent events or conversations, something 
that gives each text a kairotic exigency. It should make the text available to the widest possible public — not burying interesting things behind paywalls, not reserving content for subscribers. It should engage in practices designed to seek and maintain public attention - because, as Warner (2002) said, attention is prerequisite to creating a public. And it should make use of the Internet allegiance to sharing, encouraging authors to share sources and resources.

Can such a publication founded and run by academics without start-up funds or traditional sources of publishing revenue be feasible, potentially sustainable, and theoretically effective? A meaty question — and the topic of the project proposal and style guide to follow, in the conclusion to this dissertation. 


\section{CHAPTER V}

\section{DOUGH-TWISTING, SOCIALIZING THE TACIT, AND SETTING THE TABLE FOR OUR PUBLIC}

In The Knowledge-Creating Company, Ikujirō Nonaka and Hirotaka Takeuchi

(1995) described how a software developer working to design a home bread machine

successfully imitated the best baker in town:

To capture the tacit knowledge of kneading skill, she and several engineers volunteered to apprentice themselves to the hotel's head baker. Making the same delicious bread as the head baker's was not easy. No one could explain why. One day, however, she noticed that the baker was not only stretching but also 'twisting' the dough, which turned out to be the secret for making tasty bread. Thus she socialized the head baker's tacit knowledge through observation, imitation, and practice. (p. 63-4)

I embarked on this dissertation project with the goal of uncovering some of the doughtwisting of citation practices, the kinds of tacit knowledge that contribute to the ineffable tastiness of writing in three different communities of practice (Lave and Wenger, 1991): the popular press online magazines Slate and Newsweek, the print rhetoric and composition journals College Composition and Communication and College English, and the webtext rhetoric and composition journals Kairos and Computers and Composition Online. My distant reading methods were intended to help me enact a sort of impartial observer role, a hidden camera in the genre bakery; my interviews were intended to help me socialize the tacit knowledge that I observed, interpreting it in collaboration with the head bakers. Citing is an embedded practice with deceptive apparent self-evidence: When 
asked how they cite, authors tended to cursorily nod to their undergraduate inductions into MLA and APA style; when asked why they cited in a particular moment, they revealed much more complex understandings of citation.

Citation practices are records of research, records of thinking, records of the ways that people go about writing — constructed rhizomatically in the way that much writing is, but memorable for their articulated moments of discovery, integration, and reorientation. Writers often cannot, in retrospect, recall which pieces of writing came first, or how precisely a text developed through revision. I have found, however, that writers do often remember coming across a source, and they can sometimes relate how the source shaped or reshaped a project, or how it helped them connect ideas that otherwise seemed disparate. Other times, authors citation narratives revealed the shape of a discipline: In print, Jonathan Alexander described the way that citations of particular articles become shorthand for entire movements in the field, such as the way Yancey's (2004) CCCC chair's address has come to "stand in" for a discussion of multimodality. Citation practices can reflect writers' entry into the academic community of practice: Kelly Ritter described how she has quieted the anxious voice in the back of her head that worries she should cite more with the equanimity of experience — and with the security in her knowledge that, should reviewers "ding" her for not citing a particular piece of scholarship, she can always add it later. Citation practices sometimes reflect simultaneous rhetorical and functional purposes: In hypertext, many authors pointed to ways that a link both helped make a point, and also provided readers with a resource (as in Paul Muhlhauser's description of linking to tools such as Lightbeam and Collusion). Citation practices also affect the reception and uptake of a text: Ritter suggested that overcitation 
(particularly in "trendy" subfields) could betray novice status. Anastasia Salter

emphasized how the materiality of the link's underline can also help stress an important point. And Ben Mathis-Lilley pointed out that a link well-placed on the appropriate keywords can perhaps boost a site's Google rankings. Pushed to articulate their reasons for citing and hyperlinking, authors pointed to values, concerns, and issues of personal and disciplinary identity that stretched far beyond the individual moment.

By looking for tacit consistency within and among authors' web and print citation practices, and by encouraging writers to articulate their understandings of those practices, I have sought in this dissertation to trace the contours of a larger story about how writing is changing as it moves online. That story: Writers increasingly reflect goals of provocation, of attracting a wider and potentially global audience, of spreading a message rapidly and virally, of responding to recent events and conversations, of sharing sources and resources. Some of these shifts are a matter of degree, not kind, from the print academic normal — but others come into conflict with academic values (such as the way the goal of provocation conflicts with the value of academic equivocation and theoretical complication) and academic practices (such as the way the value of recency conflicts with years-long academic time-to-publication).

In this project, I traced citation practices in three sites: online popular press web magazines (Slate and Newsweek), key print journals in rhetoric and composition (College Composition and Communication and College English), and web journals in rhetoric and composition (primarily Kairos and Computers and Composition Online). I demonstrated some consistency in citation practices within each of the three sites, evidence of citation practices that were tacit, that have not yet been discussed or documented. In Chapter II, I 
found that most hyperlink citations in both Slate and Newsweek are attached to anchor text that is, on average, about three words and about twenty characters long. That consistent practice enables rhetorical use of hyperlinks, in that when authors break with the convention, they can call attention to the linked phrase; often, the longest hyperlink in a piece was placed over the "thesis" of the article — in the materiality of the underline (and/or bolding, color change, etc.), authors found an available means of persuasion. In Chapter III, I described how traditional print citation practices are reflective of a writer's location within the disciplinary community of practice; newcomers as a whole have a higher average citation count per article than the overall average, and authors who are in turn most-cited are overrepresented at the high and low end of the citation count spectrum. As a means of understanding referential practices in addition to citation practices, I considered the surprising consistency in our use of eponymous adjectives, a consistency that finds us often eponymizing literary high theorists and rarely eponymizing the scholars who make up the bulk of our field—reproducing the race and gender biases that structure high theory. Both these chapters used distant reading to identify consistent practices semi-autonomously, without authors' interpretations. In Chapter IV, I turned to author interpretation instead; I interrogated how authors developed internally consistent hyperlink citation practices despite never having been given explicit guidelines for using links. I found some consistent themes in their discussions of how they came to link the way they do, themes that seemed reflective of attitudes about writing online, distinct from attitudes about writing in print. All three data chapters sought to identify consistent, tacit practices, and I found such practices in each site. 
The citational consistency that I uncovered sometimes resonated with authors' conceptions of their own writing and sometimes complicated them: in Chapter II, Rebecca Schuman's sense that she did more research for her Slate posts than most other Slate authors seemed supported by her abnormally high links-per-article count; yet Ben Mathis-Lilley's sense that he linked the "topic words" of his articles was not borne out in consistency in the grammar of his link placement. In Chapter III, Kelly Ritter's sense that newcomers cite more often on average than more-established authors was supported by my data; however, my data conflicted with her sense that newcomers tend to use more citation strings than more-established authors. In Chapter IV, John Duffy's move to connect his essays to recent news events in his links did seem, as editors assured him it would, to improve the acceptance of his argument about virtue ethics; yet, in the examples of Salaita and Karega, I demonstrated that connecting an online text to recent events can lead to unexpected, sometimes unwanted uptakes. This potential for identifying both resonance and conflict between and within values and practices is perhaps the greatest strength of my mixed methods approach—corpus analysis sometimes revealed patterns authors sometimes did not recognize, and other times, trends authors described in interviews inspired me to look for confirmation or opposition in my data.

Although I had initially positioned the authors representing each of my sites and the genres they produced as distinct, I kept uncovering overlaps: Very few of my interviewees worked solely in popular press web journalism, in traditional print academic writing, or in webtextual academic writing. Most had experience with several of the genres I studied. Rebecca Schumann had been a traditionally publishing academic before moving to Slate. Jonathan Alexander had written for the most recent abortive iteration of 
CCC Online before becoming editor of CCC. Kelly Ritter reviewed for the web journal Peitho. Though Kristine Blair edits $C \& C O$, she has been writing primarily in print for quite some time. Several of the webtext authors I interviewed had worked for morepopular short-form web publications; Cate Blouke had worked for Viz and BAGNewsNotes, and both Robert Kachur and Daniel Schafer had blogged professionally, Kachur for a friend's company and Schafer for a non-profit. The fact that the authors I interviewed had such varied experiences meant that many of them had already considered, to some extent, the distinctions between writing for print and writing online; many had picked a preferred medium and had clear reasons for that preference. The fact that so many authors reported having mixed print and online experiences and quite often had reflected on the distinctions in their writing practices for one or the other made the tacit nature of hyperlink practices all the more surprising. Although writers had thought about what made online writing different from print writing, most had yet been unreflective about—or at least had not yet articulated what is different about— hyperlinking specifically.

In this consistency and complexity, I did not find the magic dough-twisting I originally sought: I found tacit practices, yes, practices that I worked to turn social in my conversations with authors. That "turning social," however, never resulted in the neat knowledge-creation loop Nonaka and Takeuchi (1995) said characterizes such an investigation of tacit practices. Perhaps this messiness stemmed from the fact that none of the practices involved - as Engeström, Miettinen, and Punamäki (1999) described the dough-twisting — were "a relatively clearly defined task" (p. 380); I was asking authors about practices that involved concerns ranging from Am I supporting this point 
adequately? to Am I citing enough scholars of color? to Do I sound too much like a graduate student? to Will this website still be around in five years, when I'm trying to get tenure? In voicing such concerns, writers demonstrate that they have, as Nancy Sommers (1980) termed it, "abstracted the standards of a reader" (p. 385) —and that abstracting results in a complex pastiche of potential imagined situations of reading, distant in time and place and understanding. Yet while I did not find the dough-twisting, embedded in those tacit practices I found some of our deepest assumptions about writing, about the way academia works, about the way the Internet (and indeed, the world beyond it) works.

This dissertation has also found that, beyond a first-year composition kind of instruction in citation formats, few writers report having ever been instructed in ideal citation practices, or in the rhetoric of citation practices, either in print or online. And in web publications, it is rare indeed for a publication to have any explicit recommendations for hyperlinking. In fact, the Kairos guidelines that prompted my dissertation study (and which I described in the Introduction, including a broad admonishment against "external links in-text to outside sources"-which, the guideline reads, "is usually discouraged") were the only explicit discussion of hyperlink practices I found in any of the online publications I considered. In the vacuum left by this lack of explicit instruction, writers both in popular press and in academic webext find themselves firmly in the realm of the tacit. They read other short-form networked Internet texts, they internalize those texts' hyperlink practices, and they imitate them.

Although the experience of developing sui generis link practices is nearly universal among web authors, and, according to several, can be somewhat generative, it also has some significant limitations. Cate Blouke demonstrated the generative potential 
of being forced to come up with a new citation style, when she described developing the concept for her scrolling live-Tweeting experience of a Harlot text in part from the experience of considering how to represent a live-Tweeted conference presentation accurately and adequately. However, a "no shared explicit knowledge" model of hyperlink citation can lead to authors relying on the group's one web programmer to be the "knowledgeable informant" or perhaps just the "benevolent dictator." Muhlhauser's coauthors deferred to him constantly in our interviews, whether he was present as a cointerviewee or not. He became the de-facto expert because of his knowledge of HTML, CSS, and JavaScript — despite having admitted freely that he had never been offered any explicit guidelines about hyperlinking. In my interview with Muhlhauser and Robert Kachur, both indicated that they had never had a conversation about when or how or why to link; Kachur said he assumed that because Muhlhauser was the partnership's digital media informant, he was following established hyperlink citation guidelines. As Kachur explained how he had deferred to Muhlhauser's expertise, Muhlhauser seemed surprised-and joked, "Is there a bus coming? Are you throwing me under it?"

In some situations, insecurity about linking may discourage academics with more experience in print from writing for the web - or at least from thinking about that writing rhetorically. I am bothered, for instance, by my finding that John Duffy did not place his own links in the work he did for $I H E$ and the Chronicle. What rhetoric and composition scholar would give editors a paragraph and a list of citations and tell them to put the links in where they see fit? The prospect seems foreign; most researchers in rhetoric and composition would, I believe, have a higher opinion of the rhetorical importance of citing parenthetically at the correct moment. Yet facing a new medium and a new genre, Duffy 
understandably deferred to the de-facto experts. In fact, I do think the Chronicle editor made more effective rhetorical decisions with Duffy's links than the IHE editor; the former made use of the materiality of the highlight by placing links over phrases such as "described the Affordable Care Act" and "compared the Tea Party," rather than simply linking authors' names. But the rhetorical work that went into deciding where to link and which words to highlight was entirely separate from Duffy. I believe every rhetorician writing for the web should place his or her own links, because of the rhetorical importance of the act of linking.

As I discussed in the introduction to this dissertation, there has been a significant lack of recent attention to the ways the hyperness of contemporary, Web 2.0 hypertextswhich Brooke (2009) termed "post-hypertextual” hypertexts, for their scant resemblance to the hypertexts that made up the majority of the work considered in 1990s hypertext theory. We blog, we write about web circulation, we change our disciplinary print citation styles to reflect the proliferation and domination of web sources; yet we have not, to this point, considered in great detail the rhetoric of that new kind of "posthypertextual" hypertext, with external but no internal links, links that are key to the fundamental grammar of Internet writing. Though I have made one foray into addressing this lack, I call still for more disciplinary attention to the writing, reading, and rhetorical effects of hyperlinking. I call for a change in the way we teach hyperlinks; we cannot present hyperlinks as if they are self-evident, because the internal struggle that web writers revealed to me (for instance, John Duffy's reluctance to "link up" his own writing, and Formo and Neary's concern that they had not linked enough) demonstrates 
that links present questions even for much more experienced writers than first-year students.

I also call, particularly in Chapter IV, for a change in disciplinary web journal practices regarding hyperlinking. Our disciplinary web journals miss out on some of the greatest affordances of webtext — and fail to align themselves with the new allegiances of web writing — by discouraging hyperlinking. It is much less possible to address a text to kairotic exigencies, to situate it within a recent ongoing online conversation, without hyperlinks to places where that conversation is happening. It is more difficult for writers to share the tools and the ideas that influenced them, in line with the web allegiance to sharing, without hyperlinks. Allowing (even encouraging) more hyperlinks will doubtless lead to more pieces with dead links — but my interviews suggest that readers and writers of web text expect occasional dead links and, thanks to the prevalence and increasing quality of archival services, do not feel stymied by them the way they once did.

This dissertation further argues that, in our research, we need to consider shortform networked web argument in more detail, more deeply and consistently than we have. The distinctions between blogs and news content is blurring. The importance of networked text is growing. The amount of money that companies put into producing consistent blog content is ballooning. And in this massive expansion, explosion of hypertext, writing studies has been primarily focused on the multimodal, visual, and social aspects of web writing. I call for renewed attention to the most basic element that makes this text hyper: Its links, its connections with other texts, and its location within the massive Internet intertext. 


\section{A Two-Pronged Pragmatic Enactment: Style Guide and The Inciter}

In the conclusion to this conclusion, I present two related pragmatic enactments of the calls I have been making throughout this dissertation: A rhetoric of hypermedia style guide, and a new kind of "research notes" short-form networked web publication in rhetoric and composition. The style guide enacts my findings; the publication enacts my style guide. Online academic journals have the potential to articulate and expand the rhetorical potential of the tacit features of web writing. The style guide that concludes this chapter is one gesture at how an online publication might begin to make conscious use of this potential, offering authors guidance in a place where none yet exists, setting a high standard for thoughtful consideration of link placement and other online rhetorical practices. The style guide is thus both the enactment of my rhetorical findings - and also, hopefully, their eventual undoing. It is intended to be a document that authors and editors of web publications could borrow from, set themselves against, and propose additions or emendations to (I imagine that to someday read, "In our hyperlinking, we take a different approach from The Inciter...” would be a very high compliment indeed).

Though the guide is primarily intended as a rhetorical thinkpiece rather than a how-to manual, I am nevertheless far more directive and direct about my recommendations in it than I would ever be in an academic publication. For example, the guide advocates, “Short sentences. Short paragraphs. Short words - don’t use five syllables where two will do. Avoid semicolons and jargon.” In an academic publication, I would never make such an unproblematized pronouncement, certainly not without a discursive foray into the histories and ideologies of academic language. To be clear, I do not wholly echo the critics of academic language: Jargon serves a variety of important 
purposes, communicating highly specialized concepts to participants within a given discourse community; complex syntactical structures, long sentences, and series of embedded clauses allow writers to align parallel ideas that might otherwise appear discrete, as in this sentence; many important theoretical advances would doubtless be difficult if not impossible to communicate in language that never ventures beyond a 10thgrade reading level, as is often journalistic preference; and Ian Barnard (2010) was right to point out that many critiques of academic language, such as Dennis Dutton's “Bad Writing" contests, seem to stem from an ideological beef with the project of critical theory rather than a legitimate interest in ferreting out purple prose. Yet even academics might rightly groan upon encountering a sentence as embedded as that last one. I wish in this style guide, as Peter Elbow (1991) proposed in his denunciation of jargon, to advocate text that is more "in sync with [our] intellectual stance"- that we should write messages in full view of their communicative context, with "senders and receivers" (p. 141). The guide thus is intended to be provocative in its unequivocality: Be bold first, and invite questions later.

That provocative unequivocality is, in turn, the primary goal of the publication I am proposing, which I am calling The Inciter. Again — the style guide will enact my research findings; The Inciter, in turn, enacts my style guide. But The Inciter also responds to another need of rhetoric and composition, which I have highlighted in this dissertation: I believe we need a short-form, networked web publication, written by rhetoric and composition experts, about our research, aimed at the public.

I entered into this research with the question, "How can Kairos and Computers and Composition Online better reach the public?" I have come to realize, however, that 
Kairos and $C \& C O$ are not public-aimed projects - and the true answers to my initial question would involve changing the nature of those publications. I would not want those publications to stop publishing the digitally creative, webtextually provocative pieces they do. I would not want them to dramatically shift their own goals. As a coder, as a believer in critically creative webtext design (and a creator of some webtexts and an editor of many others), I believe we need places that publish such things; the subfield of computers and writing needs that outlet for that critically creative web work.

I discovered that what I really wanted was not a publication of original research. We have plenty of venues for that. Rather, I wanted a publication of secondary, kairotic interpretations of original research — a publication where authors could find ways that their research speaks to current moments, current issues, and current discussions. That publication would help disseminate our research more widely, designed with rhetorical velocity (Ridolfo and DeVoss, 2009) in mind. In this moment, unlike most uses of "rhetorical velocity," I intend to imbue the term with its original sense: The way a press release is designed to be distributed rapidly and widely. We do not, in general, write press releases for rhetoric and composition research; that is essentially what The Inciter will do. Every post will pick up on research in rhetoric and composition and connect that research in accessible language to the kairotic moment. The project in this way is different from Jenn Fishman's work with the Research Exchange Index, which aims to distribute shortform versions of research among writing researchers; The Inciter will not be peerreviewed, will not be comprehensive, and will be aimed at a "major newspaper" kind of general public rather than at writing researchers. 
Of course, not all rhetoric and composition research will be amenable to this kind of rewriting. To be clear, I do not mean to suggest that all rhetoric and composition research should be instrumentally oriented in this way. As a discipline, we need theorybuilding; we need critical rediscoveries of ancient, esoteric terms of rhetoric; we need ideas that cannot be Tweeted. Yet much of our work does have broader social implications and communicative consequences, and by distributing that work in an accessible form, maybe we can reinsert ourselves into the public conversations about rhetoric and composition where we have lost control.

I have obtained a domain for this publication: theinciter.com - a name that nods to the rhetorical velocity of "gadfly" publications that I have established in Chapters II and IV, as demonstrated in the successes of Schuman's, Duffy's, Salaita's, and Karega's inciting essays and social web writings; that plays on my editorial emphasis on thoughtful and rhetorical citation practices; and that puns on the word "insider," to gesture both at the fact that I intend its posts to be authored by rhetoric and composition insiders and at the fact that the publication is not aimed at insiders.

I imagine it would serve several potential functions:

1. as a research notes-style publication, where writers could share findings from recently published work; I intend to have a regular series of posts that highlights various rhetoric and composition research that might spark public interest, with the goal of soon encouraging authors to write their own research notes;

2. as a place where writers could write short posts connecting findings from their own (or others') published work to recent news events, to take 
advantage of the quick publishing cycle of short-form text, to improve the potential for writers to connect their work to kairotic exigencies, and to ensure that any writer who succeeds in making the "mile-long strike" of academic publication I describe in Chapter IV can take advantage of that remarkable success;

3. as a place where writers can construct publics of potentially interested people that might not have access to (or the inclination to access) rhetoric and composition journals—-for example:

a. medical rhetoricians could reach people affected by medical language, as well as the people who use it;

b. rhetoricians of public policy, political language, and so on, could potentially reach people constructing policy, writing speeches;

c. compositionists who consider writing in the disciplines and English for special purposes could reach the writers of work instructions, of corporate policy, of websites;

4. as a site for analyzing media - for rhetorical analysis of newspapers and magazines, of television, of movies, of scandals, of the many more obscure kinds of writing that exist.

The Inciter will be a writing-about-writing publication, aimed at the public. It is designed to share our enthusiasm for analyzing and studying the written word—grounded in our theories of process and pedagogy, but not referent to them, not dependent upon them, not assuming understanding of them. It is intended for people who have a developing interest in language and for journalists who find themselves writing about 
writing. I hope that essays published on the site would be appealing for composition instructors to assign, yes, but it is not intended primarily to be a pedagogical resource. My intention is that all posts will be research-based investigations of language, public or political or otherwise. I am wary of the risk of academic publications turning disciplinarily introspective ${ }^{55}$; one key recommendation of the site's mission statement will be to avoid references to disciplinary jargon, to disciplinary journals, or to conferences without definition, elaboration, and contextualization.

\section{Spend Attention to Gain Attention: A Marketing Strategy}

Seeking the attention of rhetoric and composition as a field will be the first goal of the publication, to attract more writers and readers. I discovered in my writing for iFixit that the best way to gain the attention of my target audience- the companies doing interesting e-waste work, the writers doing e-waste research, and so on —was to tell them I wanted to interview them about their work for a short blog post. Thus, an axiom: You can gain attention by spending attention. Drawing on Warner's (1998) notion of creating a public by connecting a network of texts, I aim with The Inciter to construct a network of texts that imagines an interested and interesting public — but also to attract attention by highlighting particular authors' work and informing them of the posts. I imagine in the first few months of the publication, I will send many emails, informing authors that I have written a post about their work, describing the goals of The Inciter, including a draft of the post, soliciting changes, and encouraging them to write for the publication sometime.

\footnotetext{
${ }^{55}$ Harlot, for example, had a "Shorts" section that occasionally published pieces of the kind I'm describing - but by 2014 was publishing mostly reports from disciplinary conferences and summaries of articles in Harlot. These are pieces with low rhetorical velocity.
} 
Several rhetoric and composition researchers, including Michelle McMullin at Purdue University and Hugh Burns at Texas Woman's University, have expressed interest in writing for the publication. Once I have published some sample posts and prefatory material, I will "soft launch" the site, and contact them to help seed the site with more posts, before announcing a "full launch" in a series of emails to Listservs and social media posts.

\section{Risks and Challenges}

This publication, of course, would suffer from many of the potential problems authors and editors described in their discussions of other efforts at web work - it has an extraordinarily low "bus count"; in the case of most webtext journals, if one or two people were hit by a bus, the journal would go under. This in not so often the case with print journals, because publishers have a stronger institutional support system, backed both by formal documentation and the financial consistency of subscriptions. For now and the immediate future, all the embodied knowledge of The Inciter will remain with just me and my research assistant. Yet I intend to document as much of the site's functioning and intentions publicly as possible. Further, I hope to seek "non insider" participants; ${ }^{56}$ posting plain language "research notes" about other people's research, connected to public events, is a kind of legitimate peripheral participation (Lave \& Wenger 1991) that I hope will be appealing to graduate students, adjuncts, and others who may not yet consider themselves "insiders."

\footnotetext{
${ }^{56}$ Though I recognize that Harlot had significant trouble with this-even if The Inciter is just me (or just me and the 3 or 4 people who have jumped at the chance to write for me) for a long time, it will still be meeting my primary goals.
} 
Many rhetoric and composition web writers expressed dissatisfaction with the level of institutional recognition they have received for their online work, and The Inciter cannot offer much in that regard. Duffy, for instance, expressed that despite a "back pat" email from the Dean, he has not been able to "count" that work as work. Even Anastasia Salter, who has been publishing about digital pedagogy for nearly half a decade on ProfHacker, suggested that all her writing there was buried in a low-level CV line, to the apparent disinterest of committees that have reviewed her work at University of Central Florida. The Inciter would likely suffer from a similarly low level of formal institutional recognition. Nevertheless, the WPA Listserv attention to any rhetoric and compositionrelated article on The Chronicle or IHE suggests that there is disciplinary interest in, if not formal disciplinary recognition of, the kind of writing I am proposing.

For a content management system, I have selected GoDaddy's Managed WordPress Hosting because, despite my own personal quibbles with WordPress ${ }^{57}$, it is familiar to huge numbers of rhetoric and compositionists - and I hope that as the site gains attention, increasing numbers of writers will seek to be involved. While Managed WordPress Hosting allows flexibility for site designers who have knowledge of web coding, at the content management level, it looks just like a free WordPress site. Thus, selecting WordPress means that writers will need little technical know-how to write for the site; what little they will need is widely shared well-documented. Further, it is relatively inexpensive: My start-up costs were just $\$ 66$, and my site will function with no additional costs up through 25,000 visitors per month. I expect that the cost to continue would be under $\$ 100 /$ year. Though I hope eventually to seek institutional support of the publication of some variety_via university sponsorship or grant funding or advertising,

\footnotetext{
${ }^{57}$ Namely, the way it has been a key driver in the "templatization" of web design in the last decade.
} 
ideally something consistent and even not necessarily dependent on my involvementprovided the site gains the interest I hope to achieve, I should be able afford to pay that maintenance cost out of my own pocket in perpetuity.

Below, some of the initial documentation I offer on the site, to inform readers and guide potential writers, reflective of the findings of Chapters II - IV:

\section{The Inciter, A Mission Statement}

Our Posts Will

- Share research from writing studies, rhetoric, and composition with a broad public, to instruct and delight in equal measure

- Each address a single interesting "finding" in writing studies research

- Be relatively short, ranging from 275 to 3000 words

- Connect to timely events or discussions in public discourse and/or popular culture

- Share public, open-access versions of sources wherever possible (and screenshot relevant elements where not)

- Reflect short-form web publishing practices, including frequent use of Creative Commons-licensed images, and thoughtful and rhetorical link citation practices

- Be written at no more than a 12th-grade reading level, ideally closer to 7 th or 8 th, with the goal of reaching younger, non-expert, non-English-fluent readers

- Present at least one piece of research, ${ }^{58}$ either original or published, academic or otherwise

\footnotetext{
58 "Research," in this last point will be broadly defined - a rhetorical analysis of a particular piece of political discourse, for instance, would be "original research"; part of the goal of the site is to define "research" in the public eye in the way we do in writing studies, where "purely" textual research is not only accepted but highly valued.
} 
- Be copy-edited but not peer-reviewed or refereed

Our Readers Will Be

- Interested in language and writing

- Interested in what writing studies research has to offer

- Potentially global

- Potentially non-native English-speaking

- Willing to engage with complex ideas, but seeking approachable language

- Potentially teachers, potentially writing teachers - but not necessarily

We Won't Assume That Our Readers Are

- Academic

- Teachers

- English monolingual

- Familiar with any particular work or person cited, academic or otherwise

- Able to access academic literature of any kind

- Comfortable with academic jargon of any kind

- Subscribers to any particular ideology or political party

- Seeking to "steal ideas" and rush them to publication ahead of the author

Our Writers Will Be

- Willing to share their findings in brief, informative posts, with an interested, intelligent, but non-academic public 
- Volunteer, at least at the outset

- Likely unrecognized, officially, for the work they put into posts

- Committed to the publication and its potential role in improving the status and global/policy recognition of rhetoric and composition research 


\section{Rhetoric of Hypermedia, A Style Guide ${ }^{59}$}

\section{Elizabeth Chamberlain}

Maybe you're new to writing for the web. Maybe you've done some personal blogging, but you're unsure how that'll translate into more-public web writing. Or maybe you're not new to it at all, but you'd like to be more reflective about the way you write online. This rhetorical style guide is intended to provoke, to get you thinking about what Aristotle calls "the available means of persuasion" you'll find on The Inciter" ${ }^{61}$ : short-form, hyperlinked web text, aimed at a "general public." It's the kind of text that may include some embedded multimedia-but really is more about the text than about the videos or pictures that accompany it. The guide below is more thinkpiece ${ }^{62}$ than manual ${ }^{63}$, research-based but ultimately not a step-by-step "how to."

If you've ever wondered, "How long should my posts be?" or "How do I write an effective headline?" or "When and how should I hyperlink?"... well, I have, too. And to be honest, you're probably going to have more questions when you finish reading than you do right now. But they'll be questions informed by research. I hope they'll also be questions that incite you to do bold, creative, and rhetorically thoughtful things with your writing, here and elsewhere.

The inspiration and much of the content for this guide came from my dissertation work $^{64}$. That project included computerized analysis of texts on Slate ${ }^{65}$ and Newsweek ${ }^{66}$, and interviews with both academic and non-academic web authors (and some in-between, including Slate's Rebecca Schuman, an ex-academic who writes about academia ${ }^{67}$ ). The guide is also based in my experiences as a web copywriter for several businesses and as an Associate Editor of the web-based rhetoric and technology journal Kairos ${ }^{68}$.

Here's a map of the subsections, including a "Too Long; Didn't Read" (TL;DR) version of each:

\footnotetext{
${ }^{59}$ In this print version of the Style Guide, I cite using hyperlinks in footnotes that follow a piece of bolded text, to approximate the look/function of the online version.

${ }^{60}$ http://www.americanrhetoric.com/aristotleonrhetoric.htm

61 http://theinciter.com/mission.html

http://www.slate.com/blogs/browbeat/2014/05/07/thinkpiece_definition_and history roots of the word show it has long been.html

${ }^{63}$ https://owl.english.purdue.edu/owl/resource/747/01/

64 http://elizabethfchamberlain.com/work.html

$65 \mathrm{http}: / /$ slate.com

66 http://newsweek.com

67 http://www.slate.com/authors.rebecca_schuman.html

68 http://kairos.technorhetoric.net
} 
- How long should my posts be? ${ }^{69} \mathrm{TL}$;DR: Somewhere between 500 and 2500 words.

- How can I attract a wide audience $?^{70} \mathrm{TL}$;DR: Connect your post to recent news events.

- How should I sound? ${ }^{71} \mathrm{TL}$;DR: Like a friend.

- What can I learn from BuzzFeed? ${ }^{72}$ TL;DR: Long, emotional headlines about "you."

- What kind of images should I include, and when $?^{73} \mathrm{TL}$;DR: Creative Commons-licensed images - lots of them.

- When and how should I hyperlink ${ }^{74}$ TL;DR: Frequently— to strengthen an argument, to give honor to content creators.

\section{How Long Should My Posts Be?}

The ultimate rhetorical answer: As long as they need to be. Really, though, research suggests that the optimal post length depends on your goal: the text-sharing site Medium's research demonstrates that average viewer time spent per article ${ }^{75}$ rises steadily as articles get longer through a 7-minute read (or about 1,900 words), then begins to taper off. The digital marketing firm Snap found that "organic" traffic peaks between 2250 and 2500 words $^{76}$ - but social sharing is actually highest for articles over 2500 words, and their analysis suggests that the ideal socially shared web article length will continue to rise. A blog adviser at The Write Practice suggests that posts of under 275 words inspire the most comments ${ }^{77}$, but admits that they're rarely shared on social media.

My data showed that on Slate, the mean post is 639 words, and on Newsweek it's 690. So if you're trying to get more reader engagement, you should probably keep things snappy. If you want most of your readers to read the whole thing, 1,900 words appears to be the sweet spot. If you're hoping for wide distribution on social media, however, you might be better off writing an essay long enough to make a college freshman groan.

But if you write something that long, you've got to keep your reader's attention, which brings me to my next point:

\section{How Can I Attract a Wide Audience?}

One of the best ways to attract the attention of an online audience is to connect your ideas to recent, topical news events, Notre Dame English professor John Duffy told me. Duffy is the author of two short web thinkpieces on Inside Higher $\boldsymbol{E d}^{\mathbf{7 8}}$ and The Chronicle of

${ }^{69}$ \#shortform

70 \#wideaudience

${ }^{71}$ \#tone

72 \#clickbait

${ }^{73}$ \#images

${ }^{74}$ \#links

${ }^{75} \mathrm{https}: / /$ medium.com/data-lab/the-optimal-post-is-7-minutes-74b9f41509b

76 https://www.snapagency.com/blog/whatll-be-the-best-length-for-a-blog-article-in-2016-for-seo/

77 http://thewritepractice.com/blog-post-length/

78 https://www.insidehighered.com/views/2012/03/16/essay-value-first-year-writing-courses 
Higher Education ${ }^{79}$ that have both achieved an audience outside the field, even though they're really aimed mostly at writing teachers.

Duffy attributed that success to the way he connected each article to some things that had happened in the news in recent weeks. From his experience, Duffy explained, "If we can connect the things we know to kairotic moments"-a rhetorical term meaning the right and opportune time ${ }^{80}$ - "in public discourse, in popular culture, in the media and so forth, we stand a better chance of getting published [in popular press publications]."

Attention to texts, social theorist Michael Warner says, can actually create a public ${ }^{\mathbf{8 1}}$ : a public will organize itself around a network of texts when those texts capture its attention. The best way to attract a wide audience, that is, is to write texts that imagine and speak to a wide range of possible readers.

That's why I argue below for sounding like a friend:

\section{How Should I Sound?}

If your goal is to reach a wide audience, make "shorter" your mantra (especially if you're an academic like me, used to writing for other academics). Break up sentences. Don't embed them. Short sentences. Short paragraphs. Short words - don't use five syllables where two will do. Avoid semicolons and jargon. This isn't a matter of "dumbing it down" for your audience. It's about making it possible for your audience to include nonexperts, people who stumble across your writing in their Google search results.

For The Inciter, which is intended to be a publication about rhetoric with an audience that includes non-rhetoricians, it's especially important that we work hard to define our terms, never assume prior knowledge, introduce anybody we cite, and avoid getting too "inside baseball' $^{\text {\$2 }}$ with our discussions of either rhetoric or teaching. But it's also important to keep our goal of informing and delighting ${ }^{\mathbf{8 3}}$ in mind, always. We've got to be engaging, clear, and research-oriented-but never condescending. I like to imagine I'm writing an email to a friend from another field with a question about my work.

Think about the kinds of genres that you find most interesting and enjoyable to read, and think about how a research-based version of that genre might look. Some of the authors I talked to as part of my dissertation research-Paul Muhlhauser, Cate Blouke, and Daniel Schafer-presented an article they wrote together as an infographic in addition to the traditional academic text. ${ }^{84}$ Daniel Schafer explained to me that they chose an infographic with the goal of reaching an audience that might not "have the relevant training or background or whatever to just jump into an article like that." But they also

\footnotetext{
${ }^{79}$ http://www.chronicle.com/blogs/conversation/2013/12/03/the-ethics-of-metaphor-2/

$80 \mathrm{http}: / /$ www.merriam-webster.com/dictionary/kairos

81 http://castillocorrales.fr/wp-content/uploads/2013/03/Michael-Warner-Publics-Short.pdf

$82 \mathrm{https} / / /$ en.wikipedia.org/wiki/lnside baseball (metaphor)

83 http://www.goodreads.com/quotes/295935-the-aim-of-the-poet-is-to-inform-or-delight

${ }^{84}$ http://cconlinejournal.org/fall15/kairos/infographic.html
} 
recognized that even as academics, they sometimes preferred short-form texts like infographics, because they're more directed and less time-consuming.

That's why even this style guide has "TL;DR" answers to each subsection's question. If you're looking for models, don't turn to places where you usually find the research you read. Instead, take a look at what makes your "guilty pleasure" reading so entertainingfor me, that means turning to places like Slate, the Reddit forum "Explain Like I'm Five,

What Can I Learn from BuzzFeed? (or, One Weird Trick-Academics HATE It!) One of the first times I presented my dissertation research at a conference, I had picked the inciting title, “'One Weird Trick': Popular Press Strategies to Invigorate (or Infuriate) Online Open-Access Journals." I thought that suggesting professional writing teachers might learn something about writing from BuzzFeed would be provocative. And sure enough, one person who raised her hand at the end said, "I have to admit: when you first started talking, I was angry. I hate clickbait." Nobody agrees ${ }^{87}$ about how to define "clickbait" (Does the headline have to be misleading, or is simply being vague enough? What counts as "formulaic"? Does it have to use the word "you"? Does it have to be attached to vapid, useless content?). Yet everybody—hilariously, even BuzzFeed ${ }^{88}$ decries "clickbait," including many of the academics in the room at my conference presentation, who nodded appreciatively when the woman shared her clickbait ire.

"But then," my would-be critic shrugged, "you started to make a lot of sense."

\footnotetext{
${ }^{85} \mathrm{http}: / / \mathrm{www} \cdot$ reddit.com/r/explainlikeimfive

86 http://www.pbs.org/newshour/making-sense/what-you-dont-know-about-click-bait-journalismcould-kill-you/

87 http://digiday.com/publishers/many-many-ways-publishers-define-clickbait/

88 https://www.buzzfeed.com/bensmith/why-buzzfeed-doesnt-doclickbait?utm_term=.awxJN29BO\#.vIWEDWIpj
} 


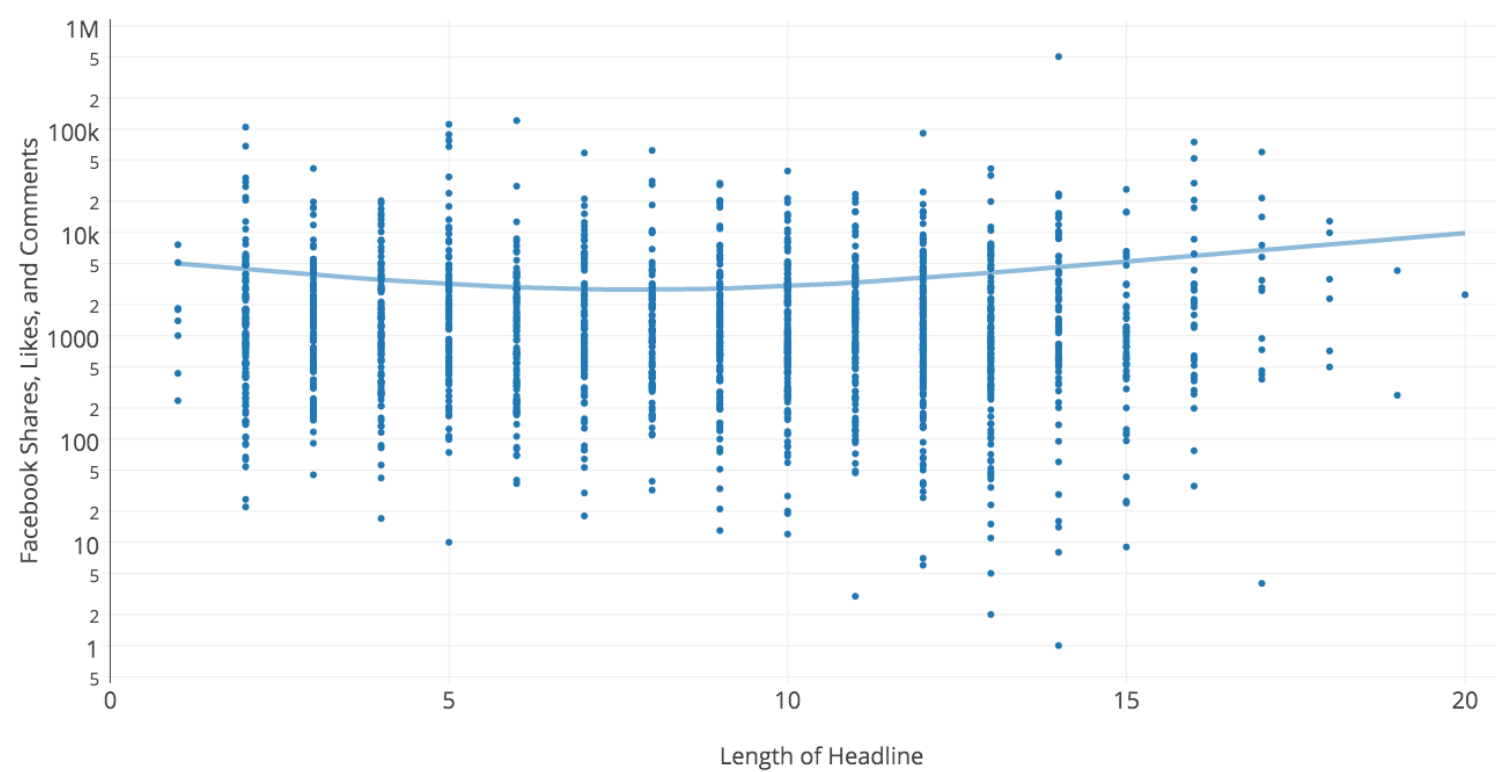

Figure 5.1: Headline length, in words, charted against Facebook shares, likes, and comments.

I shared, for instance, a graph that charted the length of Slate headlines in words against the social sharing of the post. There was a wide range of lengths, from a bunch of oneand two-word headlines through a handful of eighteen-, nineteen-, and twenty-word headlines. When I fit a trendline to the social sharing data, an interesting dip in the middle appeared (a dip that's bigger than it looks at first glance, because the y-axis is logarithmic, to make it possible to see all the social sharing data that ranged from 10 shares to over 5 million shares).

Shorter-than-average headlines and longer-than-average headlines seem to do better on social media. The lowest average shares are for nine-word titles, and the highest are for sixteen-word titles. The most successful one-word title: "Escarnope." A couple of successful super-long titles: "This Is What It's Like to See Pitch Perfect 2 with an Entire College A Capella Group" (17 words) and "A Photographer Processes Her Parents' Cancer by Documenting It in These Raw and Touching Images" (15 words).

If there is a "clickbait" formula for these long titles, it might look something like this: Be long and rambling. Make a promise about the emotional experience that the reader will have while reading the article, and don't be afraid of using adjectives or editorializing ("entire college a capella group," "raw and touching"). Refer to the article itself ("this is what it's like," "these [...] images"). 
And maybe use a pronoun or two: My data also showed that on both Slate and Newsweek, headlines that included the pronouns "I," "you," "they," or "us" had a higher-thanaverage rate of social sharing. This resonates with software engineer Max Woolf's similar social sharing analysis of BuzzFeed titles ${ }^{89}$, which found that the top five mostshared phrases were, "character are you," "[x] things only," "before you die," "is this the," and "you probably didn't."

Do I recommend you clickbait-ize your titles? Not to the extent that you mislead readers. And not to be deliberately, deceptively vague, turning them into a featureless soup of pronouns with no obvious referents.

Yet I think it's worth recognizing that readers respond to headlines that hint at the emotional experience they'll have with an article. It's worth recognizing that without the limits of strict column inches that have led to the development of newspaper headline grammar $^{90}$, online headlines can speak more conversationally to readers. You don't have to eliminate "and"s and "the"s and "be"s. You can editorialize in the way that journalism guides have railed against ${ }^{91}$ - because the assumptions of web writing are different. One of the real cultural triumphs of BuzzFeed, of Vice, of even places like the New York Times blog series is that we increasingly accept that news media has some inherent bias, and we don't expect that authors of headlines will avoid commenting upon their subjects.

Some academics have been working to achieve widespread acknowledgement that everyone is biased $^{\mathbf{9 2}}$ for nearly 30 years. Thanks maybe in part to BuzzFeed (among, of course, many other things), that's a far less controversial statement today than it was 30 years ago.

BuzzFeed has also been a serious pioneer in the way it standardized a particular kind of headline-plus-image listicle that's gained enormous traction ${ }^{93}$ :

What Kind of Images Should I Include, and When?

There are several key considerations in using images:

- How to find relevant images

- Where to use images within a post

- What kind of licenses are acceptable

- How to cite images

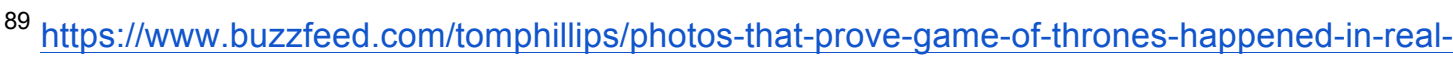
life?utm_term=.jb8yRNx69\#.edGaZDBXI

${ }_{91} \mathrm{http} / / / \mathrm{www}$. chronicle.com/blogs/linguafranca/2013/02/18/the-grammar-of-newspaper-headlines/

https://books.google.com/books?id=VSZmAQAAQBAJ\&pg=PA151\&lpg=PA151\&d\#v=onepage\&q $\& \mathrm{f}=$ false

${ }^{92}$ http://archive.boston.com/news/science/blogs/science-in-mind/2013/02/05/everyone-biasedharvard-professor-work-reveals-barely-know-our-ownminds/7x5K4gvrvaT5d3vpDaXC1K/blog.html

${ }^{93}$ https://www.wired.com/2014/01/defense-listicle-list-article/ 
Images are necessary for the layout of The Inciter: Each post is represented in the feed by a single, headlining image. That image will be a kind of metonym-it'll "stand" for your post $^{\text {94 }}$, saying something about the content within, representing the research you present. You should think carefully about that image: Do you want to represent your content literally? (e.g. If you're talking about research on college students' writing, will your picture be of an adolescent sitting thoughtfully in front of a laptop?) Do you want to represent it with common visual metaphors? (e.g. If you're talking about research on Australian writing, will your image include a kangaroo and an Australian flag?) Do you want to represent it more abstractly? (e.g. If you're talking about research on pediatricians' writing, will your image include healthy-looking kids?) Images from the research itself are great where possible and available - if you can highlight a sharplooking graph, all the better.

You may also want to include more images in the post. Consider the BuzzFeed-style listicle, with an image representing each item in the list, or the images in Cracked articles, which often make visual jokes/puns on the content of the article. You don't need to have more than one image, but subheading images can help keep readers engagedand the exercise of coming up with a visual metaphor for your content will keep your eye on the "takeaway."

A quick note on accessibility: When you include images in your post, be sure to include "alt text,"95 which is a metadata tag associated with your image that makes it possible for someone with a screenreader to understand the image. As you add an image, WordPress will prompt you to fill in that metadata. (If you forget, you can go back and edit it in.)

What kind of images to use? Where to find them? Since The Inciter is a non-commercial enterprise, you can use any image that is published in the Creative Commons ${ }^{\mathbf{9 6}}$ or in the public domain, without special permission from the creator. Know your Creative Commons licenses ${ }^{97}$, especially if you're going to be modifying or remixing any of your images: Most importantly, know anything tagged with "BY" has to be attributed to its creator.

Where to find images? Flickr is a great source of Creative Commons images ${ }^{\mathbf{9 8}}$, and because it's a site aimed at photographers, it has particularly good tools of indicating which license the creator intends. It's also far less likely than other image hosts, in my experience, to have images that have been taken without credit/attribution from photographers, shared and rehosted by a stealing stranger. It's polite but not necessary to ask the creator of a CC-licensed image if you can use their image in a post. If you find a non-CC image on Flickr that you especially like, you can always send a private message to the creator asking for permission to use it; in my experience, about half of the Flickr

${ }^{94}$ https://en.wikipedia.org/wiki/Metonymy

95 http://webaim.org/techniques/alttext/

96 https://creativecommons.org/

97 https://creativecommons.org/licenses/

98 https://www.flickr.com/creativecommons/ 
users I've contacted this way have responded, and nobody who has responded has denied the request.

Google Images also makes it possible to search for CC-licensed content: On a search results page, click "More Tools"... But there's a higher risk on Google Images that you'll come across images that have been inappropriately taken from a creator and rehosted with a CC license, without the original creator's permission or intent. Do not just accept Google Image's word that an image is CC-licensed; always click through to the page where the image came from. Consider questions like: Is it the creator's site? If not, can you tell who the creator is? Does the CC license seem credible? Obviously, at some point you have to make a judgment call — and any creator who sees the image and is unhappy with your use of it can ask you to take it down.

Attribution on The Inciter is simple: When you're filling in the metadata tag for the image, put the website source (e.g. Flickr? Google Images? Archive.org? Imgur? Photobucket?) and the author's name (if available) or username. Place a hyperlink to the URL where you found the image over the author's name.

This brings me to my next point-hyperlinking practices on The Inciter in general:

\section{When and How Should I Hyperlink?}

Be aware of the economics of hyperlinking: Because links and eyeballs are the primary currency of the web attention economy (and they translate very literally into dollars when people are seeking advertiser funding), know that when you link something, you very well may be indirectly giving money to the creator. Jeff Jarvis calls this the "link economy."99

Thus, one of the best ways you can support someone whose work you like is by linking to them. A link to their page with "good keywords" will boost their search rankings ${ }^{100}$, making it more likely that web users (even ones who don't read your site) will find them. And, of course, it increases their exposure/visibility in a more direct sense, too-your readers may click. In my dissertation research, McDaniel college English professor Dr. Paul Muhlhauser described hyperlinking as "giving honor."101

If you're talking about practices you find deplorable, however, you may be better off screenshotting than linking the source you're citing. That practice gives you some security, too, in knowing that even if the site gets changed/removed, your argument won't be invalidated. For example, many websites, including The New York Times, made the collective decision never to link to the now-defunct gossip website Gawker ${ }^{102}$ though editor Margaret Sullivan admits in that post that the Times is still figuring out how to standardize its hyperlink practices.

99 http://buzzmachine.com/2008/06/18/the-link-economy-v-the-content-economy/

$100 \mathrm{http}: / /$ www.wordstream.com/seo-keyword

101 http://paulmuhlhauser.org/

102 http://publiceditor.blogs.nytimes.com/2015/07/22/to-link-or-not-to-link-gawker-and-more/ 
Be aware of the ways that hyperlinks beckon to readers. They invite distraction in a more meaningful way than parenthetical citations do. Nicholas Carr calls links a "technologically advanced version of a footnote" — but "also, distraction-wise, a more violent form of a footnote ${ }^{\mathbf{1 0 3}}$." In 1998, Michael Miller and L. Jay Wantz said that clicking on a link often leads to "a feeling of being lost in 'cyberspace.,"104" Most Internet readers use tabbed browsing ${ }^{\mathbf{1 0 5}}$, most readers are familiar enough with hyperlinks and web browsing practices (e.g. judicious use of "open in a tab behind" and the "back" button) that they won't literally get lost by following a hyperlink. But still, having too many hyperlinks can be distracting. Readers are more likely to click on a hyperlink than follow a print citation.

Consider the persuasive force of a hyperlink, even an unclicked hyperlink. Linking a phrase indicates that it's supported by research. It says, "somebody else's work says this too." And thus it makes sense that having more links is associated both with opinion content (on Newsweek, the Opinion section has on average 3 times as many links as the other sections) and with high social sharing. My research found that pieces with 20-80 hyperlinks are, both on Slate and Newsweek, more often shared on Facebook than pieces with few links. Hyperlinked content is researched content, and often persuasive content.

How long should I make the text I where I place the link — called "anchor text"? My analysis of Slate and Newsweek found that anchor text is, on average, about 3 words and about 20 characters long. Authors usually link more than one word, but usually don't link more than a few words.

But you can use this knowledge to rhetorical effect: When pieces have an especially long hyperlink, it's often over the key idea of the article. If you can link the "thesis" of your article, the hyperlink will call attention to that idea - and the anchor text being unusually long can work in your favor. Hyperlinks essentially let you bold your thesis.

What if the link goes dead? Whenever possible, provide information in your signaling phrase that will let readers search for other versions of the source you provide. Although standard web hyperlink citation practices don't involve including authors' names or titles of articles cited, I encourage you to do so where possible. The more information you can give a reader to search for archived/alternative versions of content, the better. This is especially true for the most-key pieces of your research. Some links may be less important- "non-essential" or "throwaway" links to tools or extraneous resources - and including names of creators or titles of pieces for such links may be cumbersome or prohibitively time consuming. Don't avoid linking just because it'd take too much time to explain the source; it's okay to link without an author's name. If you discover a link has gone dead while you're working on a piece, check web archival resources such as Archive.org's WayBack Machine ${ }^{106}$ or Screenshots.com ${ }^{107}$.

103 http://www.roughtype.com/?p=1378

104 http://wwwis.win.tue.nl/ah98/Miller.html

105 https://en.wikipedia.org/wiki/Browser_wars

106 http://web.archive.org/

107 http://www.screenshots.com/ 
Making it possible to find the source behind a dead link is one good reason to make your anchor text semantic. I'm not one for strict rules, but here's one: Never put a link over just the phrase "click here"108 or "click here for more information" (except in a sentence like this one, where that phrase describes the linked content). "Click here" doesn't give your reader any sense of what's coming in the link. It doesn't make use of the rhetorical potential of the hyperlink. It doesn't provide link value to the creator (because it's not a meaningful set of keywords). And you don't need to tell your readers that a link is clickable - you can assume that, because it's a link, they know to click.

It's worth knowing, too, the anatomy of a hyperlink ${ }^{\mathbf{1 0 9}}$ : That HTTP stands for "Hypertext Transfer Protocol" and tells your browser it's looking for a web address. That including both "www" and "http"110 is usually redundant, but it's become standard practice anyway. That if a URL has a question mark or ampersand ${ }^{111}$, the stuff following is usually part of a "query string" indicating data you've put into the websiteif your goal is to link to a search page, this stuff may be necessary, but otherwise you should probably delete it. You should know that sometimes your view of a page is dependent on being logged in or having accepted a site's cookies ${ }^{112}$. It's a good idea to test a link in a cookie-free browser window (e.g. Chrome's "Incognito" mode), to make sure it will look the way you want when readers click.

The upshot? Link to support your argument. Link to give credit. Link to add value to the site you're linking. Link thoughtfully, judiciously - aware of the ways that links can distract readers but also the ways they can strengthen your writing's rhetorical force.

\section{A TL;DR for This Whole Style Guide:}

Familiarize yourself with standards and conventions of web writing, where there are standards (e.g. using images as subheading, having 3-word anchor text). You shouldn't view those conventions as strict rules - many are still in development, and even where they're not, they're usually flexible. But break convention intentionally and rhetorically. Be insightful. Be an inciter.

\footnotetext{
108 http://www.wordstream.com/anchor-text

$109 \mathrm{http}: / / g i z m o d o . c o m / w h y-a r e-u r l s-f u l l-o f-g a r b a g e-c h a r a c t e r s-1719538363$

$110 \mathrm{http}: / / \mathrm{www}$.differencebetween.net/technology/difference-between-http-and-www/

$111 \mathrm{https}: / /$ en.wikipedia.org/wiki/Query string

112 http://www.allaboutcookies.org/cookies/
} 


\section{REFERENCES}

Aarseth, E. J. (1997). Cybertext: perspectives on ergodic literature. Baltimore: JHU Press.

Allen, R. L. (2004). Whiteness and critical pedagogy. Educational Philosophy and Theory, 36(2), 121-136.

American Historical Association (1999). Who is teaching in US college classrooms? A coalition on the academic workforce study of undergraduate faculty. Retrieved from https://www.historians.org/about-aha-and-membership/aha-history-andarchives/archives/who-is-teaching-in-us-college-classrooms

Aronson, B. (2004). Improving online access to medical information for low-income countries. New England Journal of Medicine, 350(10), 966-968.

Bakhtin, M. M. (1982). The dialogic imagination. Austin: University of Texas Press.

Ball, C. E. (2006). Designerly $\neq$ readerly: Re-assessing multimodal and new media rubrics for use in writing studies. Convergence: The International Journal of Research into New Media Technologies, 12(4), 393-412.

Ball, C. E. (2014, June) Designed research: Publishing designs as scholarship. Paper presented at Design Research Society Conference, Umeå, Sweden. Retrieved from CEBall.com.

Ballif, M., Davis, D., \& Mountford, R. (2008). Women's ways of making it in rhetoric and composition. New York: Routledge.

Banks, A. (2005). Race, rhetoric, and technology: Searching for higher ground. Mahwah, NJ: Lawrence Erlbaum Associates. 
Banks, A. (2011). Digital griots: African American rhetoric in a digital age. Carbondale, IL: Southern Illinois University Press.

Barthes, R. (1970). The death of the author. In S. Heath (Ed.), Image-music-text (pp. 142-154). New York, NY: Hill and Wang.

Bennett, K. (Ed.). (2014). The semiperiphery of academic writing: Discourses, communities and practices. Berlin: Springer.

Berkenkotter, C., Huckin, T. N., \& Ackerman, J. (1988). Conventions, conversations, and the writer: Case study of a student in a rhetoric Ph.D. program. Research in the Teaching of English, 22(1), 9-44.

Berlin, J. (1988). Rhetoric and ideology in the writing class. College English, 50(5), 477494.

Berlin, J. (1988). Rhetoric and ideology in the writing class. College English, 50(5), 477494.

Bernard-Donals, M. (1994). Mikhail Bakhtin: Between phenomenology and Marxism. College English, 56(2), 170.

Bishop, W. (1999). Places to stand: The reflective writer-teacher-writer in composition. College Composition and Communication, 51(1), 9-31.

Bizzell, P. (1990). Beyond anti-foundationalism to rhetorical authority: Problems defining “cultural literacy." College English, 52(6), 661-675.

Bizzell, P. (1990). Beyond anti-foundationalism to rhetorical authority: Problems defining "cultural literacy." College English, 52(6), 661-675.

Bizzell, P., Fox, H., \& Schroeder, C. L. (Eds.). (2002). ALT DIS: Alternative discourses and the academy. Portsmouth, NH: Boynton/Cook-Heinemann. 
Blouke, C. (2011, September 20). ASU students in black face cheer on football sun devils-racist or just insensitive? Reading the Pictures. Retrieved from http://www.readingthepictures.org/2011/09/asu-students-in-black-face-cheer-onfootball-team-racist-or-just-insensitive/

Blouke, C. (2015). Borat, Sacha Baron Cohen, and the seriousness of (mock) documentary. Comedy Studies, 6(1), 4-17.

Blouke, C. M. (2015). Laughter and consequence: rhetoric and the trouble with intention in humor and identity politics (Doctoral dissertation).

Blouke, C., \& Muhlhauser, P. (2014). Pleased to tweet you. Harlot: A Revealing Look at the Arts of Persuasion, 1(13).

Boismenu, G., \& Beaudry, G. (2004). Scholarly journals in the new digital world. Calgary: University of Calgary Press.

Bolter, J. D. (1991). Writing space: The computer, hypertext, and the history of writing. Hillsdale, NJ: Lawrence Erlbaum Associates.

Botsman, R., \& Rogers, R. (2010). What's mine is yours: The rise of collaborative consumption. New York: HarperCollins.

Brent, D. (1997). Rhetorics of the web: Implications for teachers of literacy. Kairos, 2(1).

Brin, S., \& Page, L. (1997). The anatomy of a large-scale hypertextual web search engine. Infolab.stanford.edu. Retrieved from http://infolab.stanford.edu/ backrub/google.html

Broad, B. (2012) Strategies and passions in empirical qualitative research. In L. Nickoson and M.P. Sheridan, Writing studies research in practice: Methods and methodologies. Carbondale: Southern Illinois University Press. 
Brooke, C. (2008, October 2). Exactly who's been in charge for the last 10 years? Collin vs. Blog. Retrieved from http://www.collinvsblog.net/2008/10/exactly-whos-beenin-charge-fo.html

Brooke, C. G. (2009). Lingua fracta: Toward a rhetoric of new media. New York: Hampton.

Brooke, C. G. (2009). Lingua fracta: Toward a rhetoric of new media. Cresskill: Hampton.

Brown, S., Enos, T., Reamer, D., \& Thompson, J. (2008). Rhetoric Review survey of doctoral programs in rhetoric and composition. Retrieved from http://www.u.arizona.edu/ enos/

Burbules, N. C. (1998). Rhetorics of the web: Hyperreading and critical literacy. Page to screen: Taking literacy into the electronic era (102-122).

Burdick, A., Drucker, J., Lunenfeld, P., Presner, T., \& Schnapp, J. (2012). Digital_Humanities. Cambridge: MIT Press.

Burton, K. L. (2015). Eternal or ephemera? The myth of permanence in online writing. Computers and Composition, 38, 57-67.

Call for solutions. [Editorial]. (2016) Harlot of the Arts 15.

Canagarajah, S. (2014). Local knowledge when ranking journals: Reproductive effects and resistant possibilities. Education policy analysis archives, 22, 28.

Castells, M. (1996, 2000). The rise of the network society, the information age: economy, society and culture, Vol. I. Hoboken, NJ: Blackwell. 
Charles, M. (2006). Phraseological patterns in reporting clauses used in citation: A corpus-based study of theses in two disciplines. English for Specific Purposes, 25(3), 310-331.

Cohen, J. (2016). Re: [Air-L] additional pieces of bias against women in journalism [Electronic mailing list message].

Connors, R. J. (1999). The rhetoric of citation systems—Part II: Competing epistemic values in citation. Rhetoric Review, 17(2), 219-245.

Derrida, J. (1993). Aporias (T. Dutoit, Trans.). Stanford, CA: Stanford University Press.

DeWitt, S. L., \& Strasma, K. (1999). Contexts, intertexts and hypertexts. Middletown, OH: Hampton.

DeWitt, S. L., Strasma, K., \& Farr, M. (1999). Contexts, intertexts and hypertexts. Middletown, OH: Hampton Press, Incorporated.

Dobrin, S. (2002). A problem with writing (about) "alternative" discourses. Schroeder, C. L., \& Bizzell, P. (Eds.), ALT DIS: Alternative discourses and the academy. London: Heinemann.

Doctoral Consortium in Rhetoric and Composition. (2016). Members. Retrieved from https://ccccdoctoralconsortium.org/members/

Duffy, J. (2004). Letters from the fair city: A rhetorical conception of literacy. College Composition and Communication, 56(2), 223-250.

Duffy, J. (2012, March 16). Virtuous arguments. Insider Higher Ed. Retrieved from https://www.insidehighered.com/views/2012/03/16/essay-value-first-yearwriting-courses 
Duffy, J. (2012, March 16). Virtuous arguments. Insider Higher Ed. Retrieved from https://www.insidehighered.com/views/2012/03/16/essay-value-first-year-writingcourses

Duffy, J. (2013, December 13). Ethics of metaphor. The Chronicle of Higher Education. Retrieved from http://www.chronicle.com/blogs/conversation/2013/12/03/theethics-of-metaphor-2/

Duffy, J. (2013, December 13). Ethics of metaphor. The Chronicle of Higher Education. Retrieved from http://www.chronicle.com/blogs/conversation/2013/12/03/theethics-of-metaphor-2/

Edbauer, J. (2005). Unframing models of public distribution: From rhetorical situation to rhetorical ecologies. Rhetoric Society Quarterly, 35(4), 5-24.

Edbauer, J. (2005). Unframing models of public distribution: From rhetorical situation to rhetorical ecologies. Rhetoric Society Quarterly, 35(4), 5-24.

Eigenfactor. (2011). Gender composition of scholarly publishing. Retrieved from http://www.eigenfactor.org/gender/

Elbow, P. (1991). Reflections on academic discourse: How it relates to freshmen and colleagues. College English, 53(2), 135-155.

Elbow, P. (1991). Reflections on academic discourse: How it relates to freshmen and colleagues. College English, 53(2), 135-155.

Engeström, Y., Miettinen, R., \& Punamäki, R. L. (1999). Perspectives on activity theory. Cambridge: Cambridge University Press.

Enos, T. (1996). Gender roles and faculty lives in rhetoric and composition. Carbondale: SIU Press. 
Eyman, D., \& Reilly, C. (2006). The arrow and the loom: A decade of Kairos. Kairos, 11(1). Retrieved from http://kairos.technorhetoric.net/11.1/topoi/eyman/index.html

Farmer, F. (1998). Dialogue and critique: Bakhtin and the cultural studies writing classroom. College Composition and Communication, 49(2), 186.

Farmer, F. (2013). After the public turn: Composition, counterpublics, and the citizen bricoleur. University Press of Colorado.

Fields, A., Martin, L.T., Licona, A.C., \& Tilley, E.H. (2015). Performing urgency: Slamming and spitting as critical and creative response to state crisis. Kairos, 20(1). Retrieved from http://kairos.technorhetoric.net/20.1/topoi/fields-etal/index.html

Fish, S. (1989). Anti-foundationalism, theory hope, and the teaching of composition. Doing What Comes Naturally: Change, Rhetoric, and the Practice of Theory in Literary and Legal Studies, 342-55.

Flower, L., \& Hayes, J. R. (1981). A cognitive process theory of writing. College composition and communication, 32(4), 365-387.

Fordham, S. (2014). Race and beyond: Are (black) female academics ignored? Center for American Progress. Retrieved from https://www.americanprogress.org/issues/race/news/2014/08/06/95248/race-andbeyond-are-black-female-academics-ignored/

Fordham, S., \& Ogbu, J. U. (1986). Black students' school success: Coping with the "burden of 'acting white."” The urban review, 18(3), 176-206. 
Formo, K., \& Neary, K.R. (2015). High school girls soliciting feedback: Lessons from a university-to-high-school OWL. Computers and Composition Online. Retrieved from http://cconlinejournal.org/fall15/owl/index.htm

Foucault, M. (2012). The archaeology of knowledge. New York: Vintage. Gallagher, C. (2014). All writing assessment is local. College Composition and Communication, 65(3).

Gallagher, J. (2010). “As y’all know”: Blog as bridge. Teaching English in the Two-Year College, 37(3), 286.

Gallagher, J. R. (2015). The rhetorical template. Computers and Composition, 35, 1-11.

Gerstman, David. (2016, February 25). Oberlin professor claims Israel was behind 9/11, ISIS, Charlie Hebdo attack. The Tower. Retrieved from http://www.thetower.org/3012-oberlin-professor-claims-israel-was-behind-911isis-charlie-hebdo-attack/

Goggin, M. D. (1997). Composing a discipline: The role of scholarly journals in the disciplinary emergence of rhetoric and composition since 1950. Rhetoric Review, 15(2). http://doi.org/10.1080/07350199709359222

Gries, L. (2015). Still life with rhetoric: a new materialist approach for visual rhetorics. University Press of Colorado.

Halavais, A. (2006). Scholarly blogging: Moving toward the visible college. In A. Bruns \& J. Jacobs, Uses of Blogs (1st ed., pp. 117-26). New York: Peter Lang.

Harris, J. (1997). Person, position, style. Publishing in Rhetoric and Composition, 47-56. Haswell, R. H. (2005). NCTE/CCCC's recent war on scholarship. Written Communication, 22(2), 198-223. 
Herring, S. C., Scheidt, L. A., Bonus, S., \& Wright, E. (2004, January). Bridging the gap: A genre analysis of weblogs. In System sciences, 2004. Proceedings of the 37th annual Hawaii international conference. IEEE.

Hess, M. (2006). Was Foucault a plagiarist? Hip-hop sampling and academic citation. Computers and Composition, 23(3), 280-295. http://doi.org/10.1016/j.compcom.2006.05.004

Hesse, D. (2010). The place of creative writing in composition studies. College Composition and Communication, 62(1), 31-52.

Hesse, D. D. (2005). 2005 CCCC Chair's Address: Who owns writing? College Composition and Communication, 57(2), 335-357.

Hewings, A., Lillis, T., \& Vladimirou, D. (2010). Who's citing whose writings? A corpus based study of citations as interpersonal resource in English medium national and English medium international journals. Journal of English for Academic Purposes, 9(2), 102-115.

Holbrook, S. E. (1991). Women's work: The feminizing of composition. Rhetoric Review, 9(2), 201-229.

hooks, b. (1992). Yearning: Race, gender, and cultural politics.

Hurley, M. G. (2010). Remediating the professionalization of doctoral students in rhetoric and composition (Doctoral dissertation, Bowling Green State University).

Hyland, K. (1999). Academic attribution: Citation and the construction of disciplinary knowledge. Applied Linguistics, 20(3), 341-367.

Hyland, K. (2009). Academic discourse: English in a global context. A\&C Black. 
Inoue, A. (2013). Diversity, language, and possibility: Four new studies of what might be. College Composition and Communication, 64(3), 559-579.

Jarvis, J. (2011). Public parts: How sharing in the digital age improves the way we work and live. New York: Simon and Schuster.

Johnson-Eilola, J. (1997). Nostalgic angels: Rearticulating hypertext writing. Westport, CT: Greenwood Publishing Group.

Johnson-Eilola, J. (1997). Nostalgic angels: Rearticulating hypertext writing. Santa Barbara, CA: Greenwood Publishing Group.

Joyce, M. (1996). Of two minds: Hypertext pedagogy and poetics. Ann Arbor: University of Michigan Press.

Juzwik, M. (2004). Towards an ethics of answerability: Reconsidering dialogism in Sociocultural literacy research. College Composition and Communication, 55(3), 536-567.

King, M. M., Correll, S. J., Jacquet, J., Bergstrom, C. T., \& West, J. D. (2015). Men set their own cites high: Gender and self-citation across fields and over time. Working paper. Retrieved from http://www.eigenfactor.org/gender/selfcitation/SelfCitation.pdf

Kinneavy, J. L., \& Eskin, C. R. (2000). Kairos in Aristotle's rhetoric. Written Communication, 17(3), 432-444.

Kissmetrics. (2014). The science of social timing part 3: Timing and blogging. Kissmetrics Blog: A Blog about Analytics, Marketing and Testing. Retrieved from https://blog.kissmetrics.com/science-of-social-timing-3/ 
Knobloch-Westerwick, S., \& Glynn, C. J. (2011). The Matilda effect—Role congruity effects on scholarly communication: A citation analysis of Communication Research and Journal of Communication articles. Communication Research, doi:0093650211418339.

Knobloch-Westerwick, S., Glynn, C. J., \& Huge, M. (2013). The Matilda Effect in science communication an experiment on gender bias in publication quality perceptions and collaboration interest. Science Communication,35(5), 603-625. doi: $10.1177 / 1075547012472684$

Kolowich, S. (2015, August 31). Is nuance overrated? The Chronicle of Higher Education. Retrieved from http://www.chronicle.com/article/Is-NuanceOverrated-/232771/

Kopelson, K. (2008). Sp(1)itting images; or, back to the future of (rhetoric and?) composition. College Composition and Communication, 750-780.

Kopelson, K. (2015). "Know thy work and do it": The rhetorical-pedagogical work of employment and workplace guides for adults with "high-functioning" autism. College English, 77(6), 553.

Kopelson, K. (2015). "Know thy work and do it": The rhetorical-pedagogical work of employment and workplace guides for adults with "high-functioning" autism. College English, 77(6), 553.

Krause, S. (2001/2002). Where do I list this on my CV? Considering the values of selfpublished web sites. College Composition and Communication Online, 54(1). Retrieved from 
http://web.archive.org/web/20021203013345/www.ncte.org/ccc/www/2/54.1/krau se_copy.html

Krause, S. (2007). Where do I list this on my CV? Considering the value of selfpublished websites - Version 2.0. Kairos, 12(1). Retrieved from http://kairos.technorhetoric.net/12.1/topoi/krause/fivejournals.html

Kynard, C. (2016). "Pretty for a black girl": AfroDigital black feminisms and the cartographies of struggle. Watson Conference, Louisville, Kentucky.

Kynard, C., \& Eddy, R. (2009). Toward a new critical framework: Color-conscious political morality and pedagogy at historically black and historically white colleges and universities. College Composition and Communication, 61(1), W24.

Landow, G. P. (1992). Hypertext: The convergence of contemporary literary theory and technology. Baltimore: Johns Hopkins.

Lanham, R. A. (2006). The economics of attention: Style and substance in the age of information. University of Chicago Press.

Lauer, C. (2012). What's in a name? The anatomy of defining new/multi/modal/digital/media texts. Kairos: A Journal of Rhetoric, Technology, and Pedagogy, 17(1).

Lauer, J. M. (1984). Composition studies: Dappled discipline. Rhetoric Review 3(1), 2029.

Lauer, J. M. (1984). Composition studies: Dappled discipline. Rhetoric Review, 3(1), 20 29.

Lave, J., \& Wenger, E. (1991). Situated learning: Legitimate peripheral participation. Cambridge: Cambridge University Press. 
Lillis, T. M., \& Curry, M. J. (2010). Academic writing in global context. London: Routledge.

Lindquist, J. (2004). Class affects, classroom affectations: Working through the paradoxes of strategic empathy. College English, 67(2), 187-209.

Long, S. (2014, May 29). Demographic distribution: Gender of citations in $C C C, R S Q$, and $R R$ abstracts. Techna Verba Scripta. Retrieved from https:/technaverbascripta.wordpress.com/2014/05/29/demographic-distributiongender-of-citations-in-ccc-rsq-and-rr-abstracts/

Mackey, R. (2014, September 13). Professor's angry tweets on Gaza cost him a job. New York Times. Retrieved from http://www.nytimes.com/2014/09/13/world/middleeast/professors-angry-tweetson-gaza-cost-him-a-job.html

Maliniak, D., Powers, R., \& Walter, B. F. (2013). The gender citation gap in international relations. International Organization, 67(04), 889-922.

Manovich, L. (2001). The language of new media. Cambridge, MA: MIT press.

Markwell, J., \& Brooks, D. W. (2003). "Link rot" limits the usefulness of web based educational materials in biochemistry and molecular biology. Biochemistry and Molecular Biology Education, 31(1), 69-72. Retrieved from http://onlinelibrary.wiley.com/doi/10.1002/bmb.2003.494031010165/full

Mat783 (Creator). (2010). Christian world map [Map], Retrieved from https://en.wikipedia.org/wiki/File:Christian_world_map.png Mathieu, P. (2005). Tactics of hope: The public turn in English composition. Portsmouth, NH: Boynton/Cook Publishers. 
Matsuda, P. K., \& Tardy, C. M. (2007). Voice in academic writing: The rhetorical construction of author identity in blind manuscript review. English for Specific Purposes, 26, 235-249. http://doi.org/10.1016/j.esp.2006.10.001

Miller, C. R. (1992). Kairos in the rhetoric of science. A rhetoric of doing: Essays on written discourse in honor of James L. Kinneavy, 310-27.

Miller, C. R., \& Shepherd, D. (2009). Questions for genre theory from the blogosphere. In J. Giltrow \& D. Stein, Genres in the Internet: Issues in the theory of genre (pp. 263-290). Amsterdam: John Benjamins.

Miller, S. (1993). Textual carnivals: The politics of composition. Carbondale, IL: SIU Press.

Miller, S. (1993). Textual carnivals: The politics of composition. Carbondale: SIU Press. Moretti, F. (2005). Graphs, maps, trees: abstract models for a literary history. London: Verso.

Mortensen, P. (1998). Going public. College Composition and Communication, 50(2), 182-205.

Mortensen, P. (1998). Going public. College Composition and Communication, 50(2), $182-205$.

Mueller, D. (2012). Grasping rhetoric and composition by its long tail: What graphs can tell us about the field's changing shape. College Composition and Communication, 64(1), 195-223.

Mueller, D. (2012). Grasping rhetoric and composition by its long tail: What graphs can tell us about the field's changing shape. College Composition and Communication, 64(1), 195-223. 
Muhlhauser, P., Blouke, C., \& Schafer, D. (2015). May the \#Kairos be with you.

Computers and Composition Online. Retrieved from

http://cconlinejournal.org/fall15/kairos/

National Center for Education Statistics. (2015). Characteristics of postsecondary faculty indicator, May. (2015). Retrieved from

https://nces.ed.gov/programs/coe/indicator_cuf.asp

Nealon, J. T. (1997). The ethics of dialogue: Bakhtin and Levinas. College English, 59(2), 129-148.

Nicotra, J. (2009). "Folksonomy" and the restructuring of writing space. College Composition and Communication, 61(1), W259.

Nielsen, J. (1999, November 28). Usability as barrier to entry. Nielsen Norman Group: Evidence-Based User Experience Research, Training, and Consulting. Retrieved from https://www.nngroup.com/articles/usability-as-barrier-to-entry/

Nonaka, I., \& Takeuchi, H. (1995). The knowledge-creating company: How Japanese companies create the dynamics of innovation. Oxford: Oxford University Press.

North, S. M. (1984). The idea of a writing center. College English, 46(5), 433-446.

North, S. M. (1987). The making of knowledge in composition: Portrait of an emerging field. Upper Montclair, NJ: Boynton/Cook Publishers.

Okpala, J. C. (2010) Rhetorical education and prophetic imagination. College English, $72(5), 539-544$.

Pecorari, D. (2010). Academic writing and plagiarism: A linguistic analysis. London: A\&C Black. 
Pedersen, A. M. (2010). Negotiating cultural identities through language: Academic English in Jordan. College Composition and Communication, 62(2), 283-310.

Pirate Parties International. (2016). Find your regional party. PP-International.net. Retrieved from https://pp-international.net/pirate-parties/

Prendergast, C. (1998). Race: The absent presence in composition studies. College Composition and Communication, 50(1), 36-53.

Purdy, J. P. (2009). When the tenets of composition go public: A study of writing in Wikipedia. College Composition and Communication, 61(2), W351.

Rhodes, J., and J. Alexander. (2015). Techne: Queer meditations on writing the self. Logan, UT: Computers and Composition Digital Press/Utah State University Press. Retrieved from http://ccdigitalpress.org/techne

Rickly, R. (2007). Messy contexts: The required research methods course as a scene of rhetorical practice. In McKee, H. A., \& DeVoss, D. N. (Eds.). Digital writing research: Technologies, methodologies, and ethical issues. Middletown, $\mathrm{OH}$ : Hampton Press.

Ridolfo, J., \& DeVoss, D. N. (2009). Composing for recomposition: Rhetorical velocity and delivery. Kairos: A Journal of Rhetoric, Technology, and Pedagogy, 13(2), $\mathrm{n} 2$.

Ridolfo, J., \& DeVoss, D. N. (2009). Composing for recomposition: Rhetorical velocity and delivery. Kairos: A Journal of Rhetoric, Technology, and Pedagogy, 13(2). Retrieved June 4, 2016, from http://kairos.technorhetoric.net/13.2/topoi/ridolfo_devoss/velocity.html Ritter, K. (2012). From the editor. College English 75(1), 9-15. 
Robillard, A. E. (2006). "Young Scholars" affecting composition: A challenge to disciplinary citation practices. College English, 68(3), 253-270.

Rogers, K. (2016, August 3). Oberlin professor accused of anti-semitic remarks is placed on paid leave. New York Times. Retrieved from http://www.nytimes.com/2016/08/04/us/oberlin-professor-accused-of-antisemitic-remarks-is-placed-on-paid-leave.html

Rossiter, M. W. (1993). The Matthew/Matilda effect in science. Social studies of science, 23(2), 325-341. doi:10.1177/030631293023002004, ISSN 0306-3127

Rouzie, A. (2001). Conversation and carrying-on: Play, conflict, and serio-ludic discourse in synchronous computer conferencing. College Composition and Communication, 53(2), 251.

Royster, J. J. (1999). Remarks to the Coalition of Women Scholars. CCCC. Atlanta, Georgia.

Salaita, S. G. (2005). Ethnic identity and imperative patriotism: Arab Americans before and after 9/11. College Literature, 32(2), 146-168.

Saldaña, J. (2015). The coding manual for qualitative researchers. Sage.

Saldaña, J. (2016). The coding manual for qualitative researchers. Los Angeles: Sage.

Salter, A. (2015). Alice in dataland 2.0. Kairos, 20(1). Retrieved from http://kairos.technorhetoric.net/20.1/inventio/salter/

Salter, A. (2016). Making comics as schoalrship. ProfHacker: The Chronicle of Higher Education. Retrieved from http://www.chronicle.com/blogs/profhacker/makingcomics-as-scholarship/61579 
Salter, A., \& Blodgett, B. (2012). Hypermasculinity \& dickwolves: The contentious role of women in the new gaming public. Journal of broadcasting \& electronic media, 56(3), 401-416.

Savonick, D., \& Davidson, C. N. (2015). Gender bias in academe: An annotated bibliography of important recent studies. HASTAC.org. Retrieved from https://www.hastac.org/blogs/superadmin/2015/01/26/gender-bias-academeannotated-bibliography-important-recent-studies

Schilb, J. (2012). From the editor. College English, 74(3), 213-2014.

Shirky, C. (2010). Cognitive surplus: Creativity and generosity in a connected age. Penguin UK.

Shor, I., \& Freire, P. (1987). A pedagogy for liberation: Dialogues on transforming education. Greenwood Publishing Group.

Smitherman, G. (1999). CCCC's Role in the Struggle for Language Rights. College Composition and Communication, 50(3), 349-376.

Smitherman, G., \& Villanueva, V. (2000). Language knowledge and awareness survey conducted by CCCC Language Policy Committee. Retrieved from http://www.ncte.org/library/NCTEFiles/Groups/CCCC/Committees/langsurvey.p $\underline{\mathrm{df}}$

Sommers, J. (2008). New voices. Teaching English in the Two-Year College 35(3), 237. Sommers, N. (1980). Revision strategies of student writers and experienced adult writers. College Composition and Communication, 31(4), 378-388.

Spellmeyer, K. (2012). Saving the social imagination: The function of the humanities at the present time. College English, 74(6), 567-587. 
Swales, J. (1990). Genre analysis: English in academic and research settings. Cambridge University Press.

Swales, J. (1996). Occluded genres in the academy. Academic writing: Intercultural and Textual issues (45-58). Amsterdam/Philadelphia: John Benjamins.

Swales, J. M. (1981). Aspects of article introductions. Language Studies Unit, University of Aston in Birmingham.

Takayoshi, P. (2015). Short-form writing: Studying process in the context of contemporary composing technologies. Computers and Composition, 37, 1-13.

Thaiss, C., \& Zawacki, T. M. (2006). Engaged writers and dynamic disciplines: Research on the academic writing life. Portsmouth, NH: Boynton.

The New London Group. (1996). A pedagogy of multiliteracies: Designing social futures. Harvard educational review, 66(1), 60-93.

The pirate party is now measured as the biggest political party in iceland. (2015, March 19). Visir. Retrieved from http://www.visir.is/the-pirate-party-is-now-measuredas-the-biggest-political-party-in-iceland/article/2015150318848

Thompson, P., \& Tribble, C. (2001). Looking at citations: Using corpora in English for academic purposes. Language learning and technology, 5(3), 91-105.

Treem, S. (Writer), \& Joel Schumacher (Director). (2013, February 1). Chapter 5 [Television series episode]. In K. Riddington (Producer), House of Cards. Los Gatos, CA: Netflix.

Tyler, D. C., \& McNeil, B. (2003). Librarians and link rot: A comparative analysis with some methodological considerations. Portal: Libraries and the Academy, 3(4), 615-632. Retrieved from 
http://digitalcommons.unl.edu/cgi/viewcontent.cgi?article=1067\&context=library science

University and College Union (Great Britain)(UCU). (2012). The position of women and BME staff in professorial roles in UK HEIs.

Walker, P. (2016). A rhythmic refrain: Britain's Mass-Observation as rhetorical assemblage. Rhetoric Review, 35(3), 212-225.

Walker, P. (2016). A rhythmic refrain: Britain's Mass-Observation as rhetorical assemblage. Rhetoric Review, 35(3), 212-225.

Warner, M. (2002). Publics and counterpublics (abbreviated version). Quarterly Journal of Speech, 88(4), 413-425.

Warner, M. (2002). Publics and counterpublics (abbreviated version). Quarterly Journal of Speech, 88(4), 413-425.

Warner, M. (2002). Publics and counterpublics (abbreviated version). Quarterly Journal of Speech, 88(4), 413-425.

Weisser, C. R. (2002) Moving beyond academic discourse: Composition studies and the public sphere. SIU Press.

Weisser, C. R. (2002). Moving beyond academic discourse: Composition studies and the public sphere. Carbondale, IL: SIU Press.

Wetherbee, B. (2015). Picking up the fragments of the 2012 election: Memes, topoi, and political rhetoric. Present Tense, 5(1).

Wolfers, J. (2015, Nov. 11). Even famous female economists get no respect. New York Times: The Upshot. Retrieved from 
http://www.nytimes.com/2015/11/12/upshot/even-famous-female-economists-getno-respect.html

Yancey, K. B. (2004). Made not only in words: Composition in a new key. College Composition and Communication, 56(2), 297-328.

Yancey, K. B. (2004). Made not only in words: Composition in a new key. College Composition and Communication, 56(2), 297-328.

Yancey, K. B. (2009). Writing in the 21st Century. National Council of Teachers of English.

Yergeau, M. (2009). Aut(hored)ism. Computers and Composition Online. 


\section{APPENDIX A: CITATION-CATEGORIZING PROTOCOL}

\section{Citation-Categorizing Protocol \\ GETTING IT RUNNING}

(All of this is already set up on the laptop. But this is what you'll do if you ever accidentally close the window or if the computer crashes / has to be restarted.)

1. In Terminal, navigate to the CCC directory.

a. Open Terminal: click on the magnifying glass in the upper right and type "Terminal."

b. At the command prompt (it will say "MacBookPro-B:

tech\$"), type "cd Desktop/CCC" ("cd" means "change directory") If it worked, the command prompt will now say, "MacBookPro-B:CCC tech\$"

2. In Terminal, open three tabs. (Command $+\mathrm{T}$ )

a. In the first, type "sudo mongod" and hit enter. When it asks you for a password, type english\#1 and hit enter. This starts the database program.

b. In the second, type "mongo" and hit enter. This is the mongo "shell"-would let you interact with the database (though you won't need to). It should say, "MongoDB shell version: 2.6.10," and have a couple warnings. The last line will say, "** WARNING: soft rlimits too low." If you see that, you know the database is running correctly.

c. In the third, type "node index.js." The Terminal should print "listening on *:3000"-this means it's working.

3. In Chrome, put "localhost:3000" into the address bar. This should bring up the categorizer.

CATEGORIZING

1. Once you've found the first unconfirmed parenthetical (the last line on the page should say, "Confirmed? no"),

2. In the text input box, check how many citations are in the parenthetical.

a. If the number is correct, hit "Enter," and the program should jump to the next citation.

b. If the number is incorrect, type the correct number and hit

"Enter."

c. If you realize you've made a mistake, you can go back by either typing "b" or hitting "back." You can navigate forward through parentheticals with "n" or "next." NOTE: neither "back" nor "next" will confirm a citation count.

3. When you reach the end of an article and are in the References list, do not count any parentheticals as citations-i.e. input "0" for any parenthetical after the References list begins.

Anticipated Questions:

What counts as a citation?

Count it as a citation if it includes a page number, a title, an author's name, or the year a work was published.

Do two works by the same author count separately?

e.g. "(of Sylvia Plath's "Lady Lazarus" and "Ariel")" 
YES! The one above counts as 2.

(Plath "Lazarus" and "Ariel”; Woolf, "To the Lighthouse"; Frost)

4

How does one work quoted in another work count?

e.g. "(Wexler, qtd. Wesling, "Prosodies" 164)."

This counts as 1 -even if the two works have separate page numbers.

How do I know if two authors joined with an "and" count for 1 or 2 ?

e.g. " (For a good introduction to casuistry, see Jonsen and Toulmin; for a deeper probing, with special reference to the Romantic period, see Chandler.)

Use your best judgement. You don't have to scroll all the way down to the References list to check; that will slow you down too much. In this case, because the sentence uses the phrase "a good introduction," that seems to suggest "Jonsen and Toulmin" are coauthors of one work. So this parenthetical would count for 2: "Jonsen and Toulmin" and "Chandler."

\section{Does it count if it's a reference to an author without reference to a particular} work?

e.g. "Of course, if one takes the controversial texts to be Bakhtin's, one reads a very different (more obviously Marxist) author."

YES!

Does it count if it begins with a word/phrase like "see," "cf.," or "also consider," or "for a deeper understand of $X$, see"?

e.g. "...about responsibility, about "Teaching and Learning to Give" (see his The Gift of Death). Closer to home, a number of teachers of literature..."

YES!

What if the "automatic categorization" is clearly wrong?

Don't worry about it. I know it's often incorrect.

Why does it say there are 3 citations every time a parenthetical has the phrase "emphasis added"?

Honestly, I'm not totally sure; it's a bug. But correct it to 1 when you see it.

How do I count several chapters in the same book, listed separately?

If they're by the same author, count it as 1 . If they're by separate authors, count the authors.

What if both the chapter and the name of the whole book are named in the citation?

Count it as 1.

What about a super-long thing that names a couple of authors?

e.g. "(As I mentioned earlier, the word "useful" is itself deeply ambiguous. As a "pragmatic pluralist" who considers William James almost a saint, I do cringe when he succumbs to the phrase "cash value" to cover usefulness. When the useful is reduced to the free-market kind of utilitarianism, I go beyond cringing to passionate refutation. So did John Stuart Mill; throughout much of his later work he insisted that for Utilitarianism 
to work, it depended on the dominant presence of "men of noble character"-human beings judging the "useful" in genuinely ethical terms. See his chapter "Infirmities and Dangers" in Utilitarianism.)"

Count the authors. Here, there are 2: "William James" and "John Stuart Mill."

What about something that references two texts but only names one author? e.g. "On the one side of the ethical crowd, too many moralists attempt to impose a mechanical code, like the ten commandments, or the twelve virtues of the Boy Scout oath I once chanted, or Stephen Covey's Seven Habits of Highly Effective People. (This simplistic book, translated into twenty-eight languages, has sold over ten million copies. For an excellent expose of the dangers in such coding, see Wolfe.)"

Count the number of texts referenced. Here, it's 2 ("This simplistic book" > Covey; "Wolfe").

\section{What if it names a literary character but not a particular work?}

e.g. "tend to discuss it in terms of what T. S. Eliot memorably described as "the ghost of some simple meter . . lurk[ing] behind the arras" (conflating Polonius with the ghost of Hamlet's murdered father)"

Count it as 0 .

What if there's a quote in the parenthetical from a work, but no page number or name?

Count it as 0 .

What if it's the name of an organization?

Count it as $\mathrm{O}$.

What if there's a big long chunk of text, or the punctuation looks really off?

Count as many authors' names or page numbers as possible. Use your best judgment.

What if it names a couple people, then says "and more" or "among others"? e.g. "to twentieth-century New Humanism (in the moral urgency of More, Babbitt, and others)"

Just count the authors' names. So here, 2.

What about a court case?

e.g. "The early decisions, including well-known cases involving attempted biographical uses of letters by J. D. Salinger and L. Ron Hubbard, were not encouraging. In the first case the talismanic "unpublished" character of the letters won out over the biographer's "fair use" claim, even though the letters were readily available to researchers in academic libraries (Salinger v. Random House)."

Count it as 1.

Do I count it if it's the title of a movie/TV show?

YES! But try not to count it if it's the name of an actor or character in the movie/show.

Do I count it if it's just taking a term/phrase from an author, not citing a whole work?

e.g. "significant how little this aspect of the reception of the novel was discussed before various "resisting readers" (to borrow Judith Fetterley's term)"

YES! 
Do I count tables and figures as citations?

e.g. "However, in nearly 30 percent of the articles, there were only occasional references to difference issues, and in about 8 percent there were no references at all (see Table 3 for complete results)."

NO! Unless the author appears to be referencing a table in another text. (If you're not sure, count it as 0.)

What if something cites several editions of the same work?

e.g. "Curiously, however, the third (1973), fourth (1979), and fifth (1985) editions

announced inside the front cover that a "companion volume" entitled Masterpieces of the Orient was available as a supplement to the main anthology."

Count each as 1. 


\section{APPENDIX B: QUESTIONS FOR FORMO AND NEARY}

\section{WEB WRITING IN GENERAL}

- What background in web writing do each of you have?

- Do you blog? When/how did you begin blogging? Have you continued? If so, what's changed about your web writing since you started?

- What kinds of models did/do you follow?

- Do you remember anything about your process of becoming aware of web writing conventions (e.g. misadventures, missteps, experiments)?

- How is writing for $\mathrm{C} \& \mathrm{CO}$ similar to / different from ... writing for a blog?

... writing for a more traditional academic print publication?

- Have you taught web writing in composition classes? If so, in what way? What's been most/least successful? How has your pedagogy drawn on your experiences writing for $\mathrm{C} \& \mathrm{CO}$ ?

\section{CITATIONS AND WEB CITATIONS}

- Is there a difference between a hyperlink and a parenthetical citation, either in reading or writing?

- If so, what/why/how? If not, why not?

- What do you see as the rhetorical functions of citation in academic writing?

- (How) Do the rhetorical functions of hyperlinking in web writing differ?

- Has anyone ever given you explicit rules for how/when/why you should cite, either in print or on the web? e.g. "You should have at least one citation per paragraph." "Don't string your quotes too close together." "Never put a hyperlink over a phrase longer than five words."

\section{HIGH SCHOOL GIRLS SOLICITING FEEDBACK:}

- Let's talk about the first paragraph in the "research and methods" section:

"The issues these girls identify contribute to two evolving fields: high school OWLs and writers as solicitors of feedback. High school writing center research has been emerging for over twenty-five years; the scholarship addresses the strategies that incipient writing centers can implement to meet high school writers' distinct needs.[4] While high school writing center research represents a 
small collection, even less research focuses on high school writing centers and technology — high school OWL research represents a meager strand in this scholarship. The available research concerning high school writing centers and technology establishes that writing centers have always included technologies from pen and paper to complex computer systems. Significantly, these scholars remind writing center directors to evaluate the digital technologies employed in the writing centers or on OWLs. Work by Inman (2006); Childers (1995, 2003); and Childers, Jordan, and Upton (1998) argues these technologies should be peripheral to writing center or OWL sessions - the digital tools should not dictate the curriculum but should enhance how high school writers learn to think, write, and revise."

- That first footnote, [4], says, "[4] See Barnett (2006); Childers (1995, 2003, 2006); Farrell (1989); Nixon-John (1994); and Fels and Wells (2011)." Later on in the same paragraph, you have another citation string — but this time, you don't footnote it. You have a mix like this, throughout, sometimes footnoting, sometimes including in text. Could you talk through your thought process for which type of citation goes where?

- Why footnote it in one place and put it in-text in the other?

- What purpose do you imagine the footnote serving?

- Could you have reversed them, put the first string in-text and the other in a footnote?

- You don't have a whole lot of external hyperlinks, but on "OWL Design," you link to Nancy Sommers's "Beyond the Red Ink." In "Heeding Lessons," you link to the Project Tomorrow Speak Up initiative. In your references list, you have a couple other hyperlinks.

- Do you remember why you included these particular hyperlinks?

- I've heard a lot of people worry about the use of hyperlinks in academic writing, because they're so difficult to maintain and so often die. Was that something you worried about, in including hyperlinks? If so, do you have any ideas about how web text authors or publishers might combat that problem?

- Was not including more hyperlinks a conscious/intentional decision?

- Although you don't have many external hyperlinks, you've hyperlinked the piece internally a lot. Could you talk through your decision to do that a little?

- Let's look at the last paragraph in your "Introduction: 
We study Courtney and her OWL-using peers because these girls' words offer invaluable lessons about writers as feedback solicitors and receivers. Based on what our interviewees revealed, we identified five limitations of this OWL for feedback solicitors. Reflecting on their OWL experiences, girls clarified what they expected of this digital response site and its ability to strengthen their agency in soliciting and negotiating feedback. Moreover, we contend that the inherent patterns within these girls' expectations can help develop an OWL response pedagogy, thereby strengthening dialogic digital sites for writers soliciting feedback. Here, we first review the literature on both high school OWLs and writers as solicitors of feedback. We then describe the CSUSM OWL's design and theoretical framework. Next, the girls speak, for themselves and each other, through representative video clips in the categories we uncovered, and we explore what the girls' ideas imply. Finally, we consider what these limitations might suggest for the next version of the CSUSM OWL.[3]

- I'm really interested in why people choose to put hyperlinks over particular pieces of text; I think it's an aspect of web citation that often goes undiscussed. It seems like you prefer to put hyperlinks over nouns.

- Why, for example, do you link "five limitations" instead of "we identified"? Do you think it would've had the same force if you'd liked "we identified" instead?

- Why "OWL response pedagogy" instead of "we contend" or "help develop"? "CSUSM OWL's design" instead of "We then describe"? 


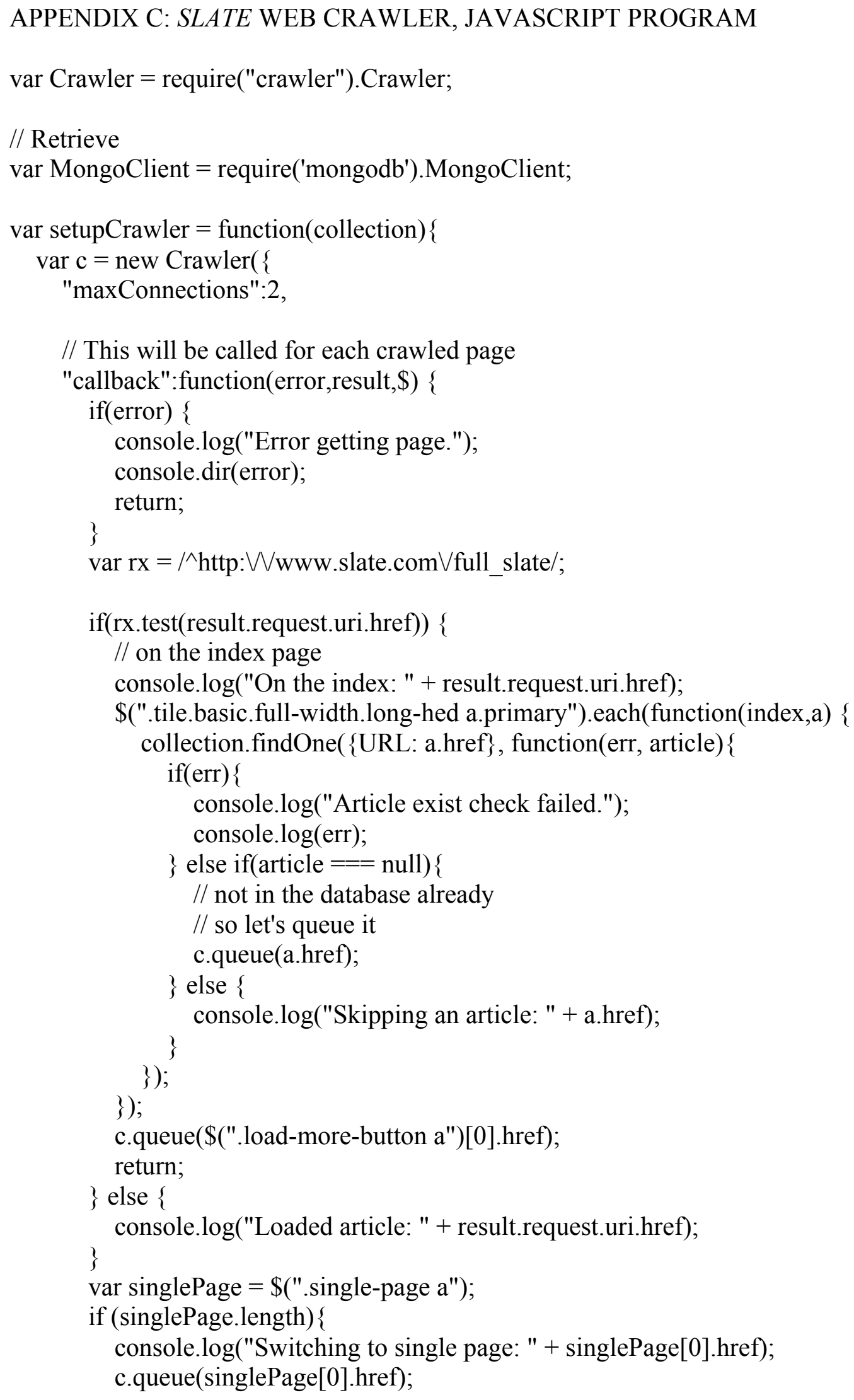




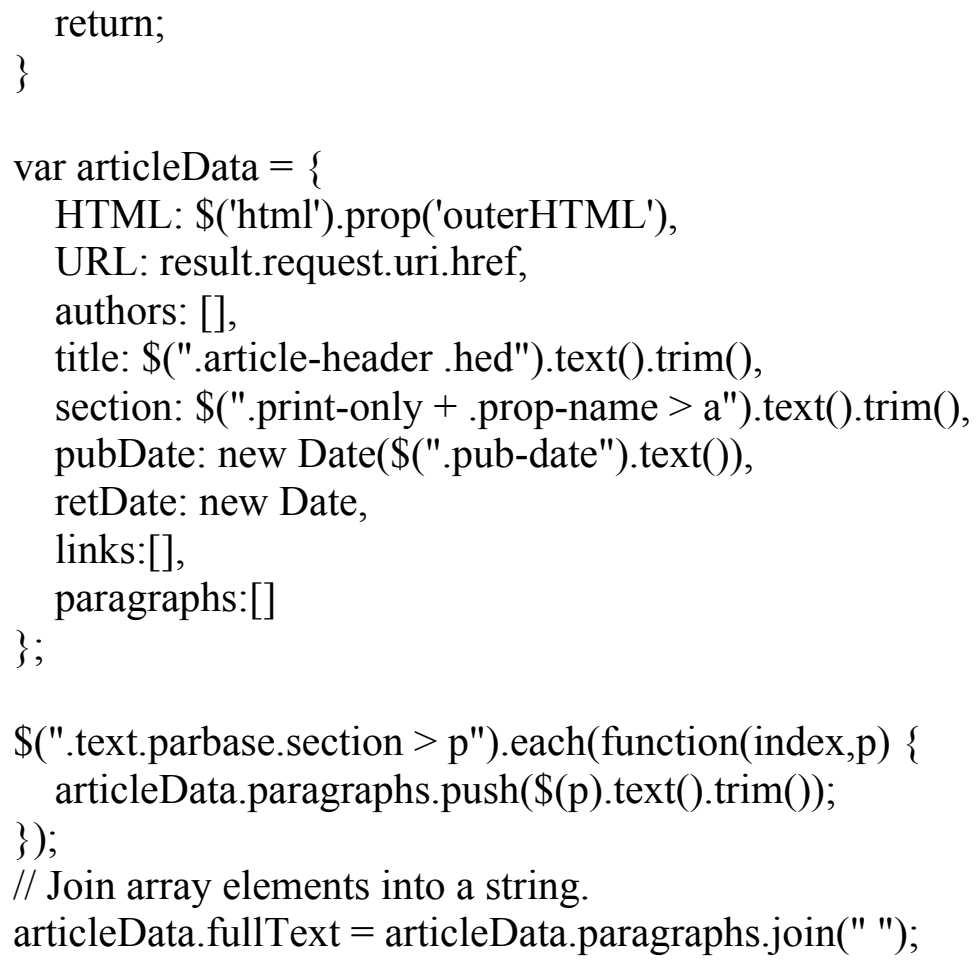

// Then turn all new lines into spaces (with replace function). articleData.fullText = articleData.fullText.replace(/[\r\n]/," ");

// Then turn all double spaces into single spaces (replace). articleData.fullText = articleData.fullText.replace $(\wedge s \backslash s+/, "$ ");

// Then trim off the final space at end.

articleData.fullText $=$ articleData.fullText.trim();

// Then count words with string.split.

articleData.wordCount = articleData.fullText.split(" ").length;

// fill authors array

\$("\#main_byline a").each(function(index,a) \{

articleData.authors.push(\$(a).text().trim());

\}$)$

// \$ is a jQuery instance scoped to the server-side DOM of the page

$\$($ ".body a").each(function(index,a) \{

var text $=\$($ a).text ()$\cdot \operatorname{trim}()$;

if(text !== "More..." \&\& text !== "Join In" \&\& text !== "" \&\& text !== undefined) \{

articleData.links.push $(\{$

text: text,

href: a.href, 


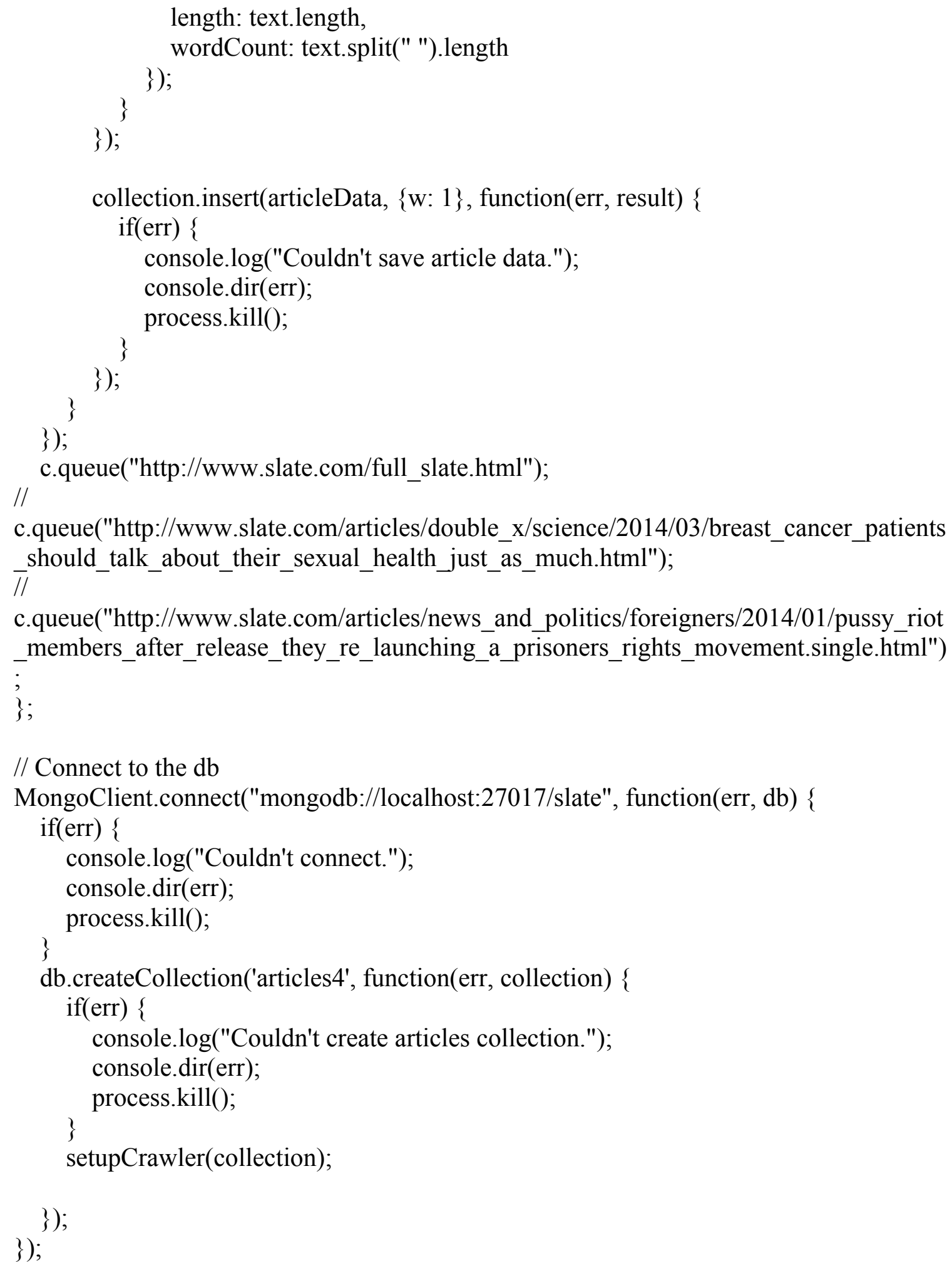




\section{APPENDIX D: SLATE LINK COUNT PER WORD COUNT ANALYSIS JAVASCRIPT PROGRAM}

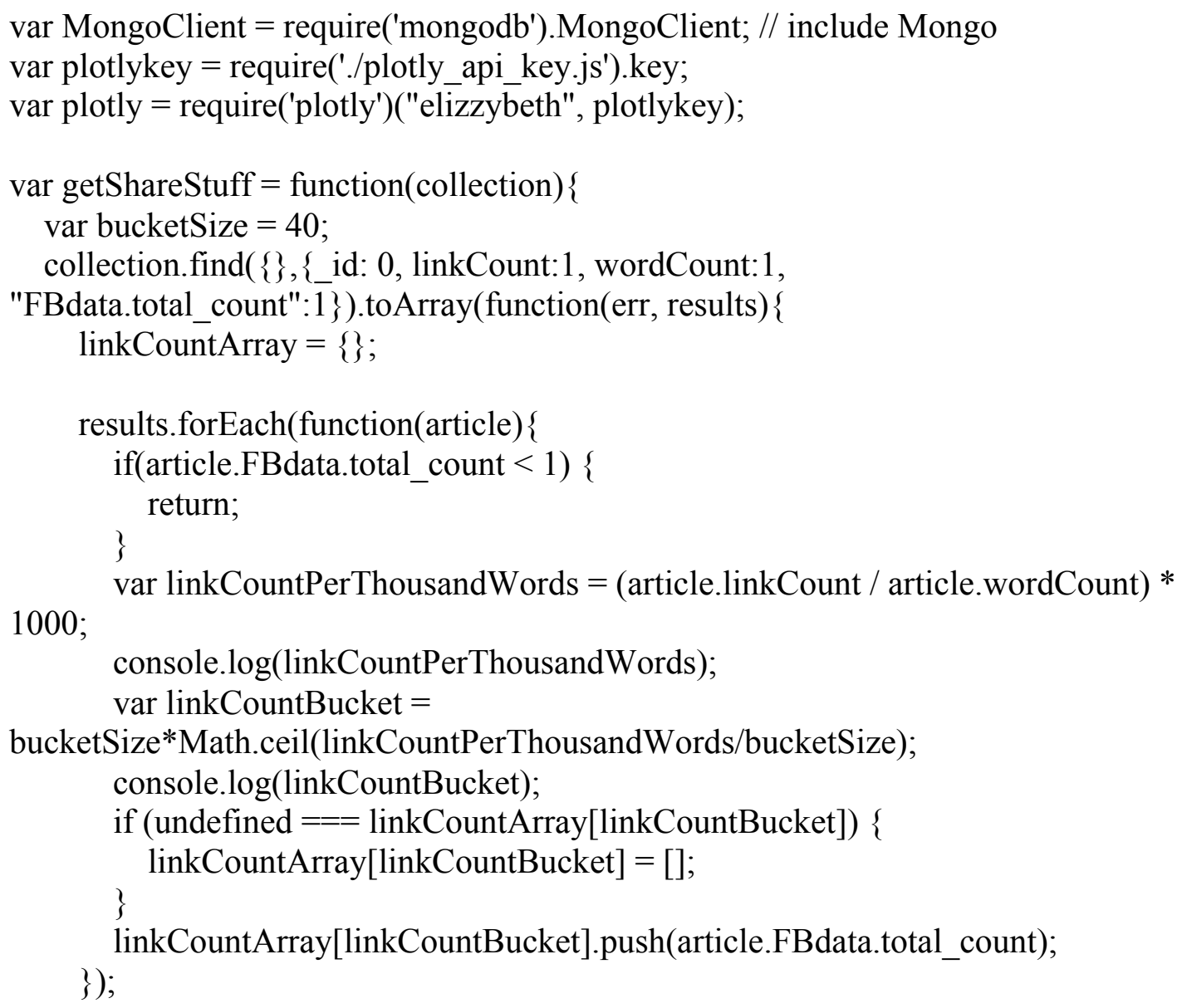


console. $\log ($ data $)$;

var graphOptions $=\{$ filename: "Slate-link-count-per-word-by-share-countbucketed", fileopt: "overwrite", layout: \{

title: "Slate: Does higher link concentration correlate with more social sharing?",

xaxis: \{

title: "Links per Thousand Words",

\},

yaxis: \{

title: "Facebook Shares, Likes, and Comments", type: 'log'

\},

type: 'box',

showlegend: false

\}

; ;

plotly.plot(data, graphOptions, function (err, msg) \{

console. $\log (\mathrm{err})$;

console. $\log (\mathrm{msg})$;

\}$)$;

\}$)$

\}

MongoClient.connect("mongodb://localhost:27017/slate", function(err, db) \{ if(err) \{

console. $\log ($ "Couldn't connect.");

console.dir(err);

\}

process.kill();

db.createCollection('articles4', function(err, collection) \{

if(err) \{

console. $\log ("$ Couldn't create articles collection.");

console.dir(err);

\}

process.kill();

getShareStuff(collection);

\}$)$

\}$)$ 


\section{APPENDIX E: Slate Crawler Flow Diagram}

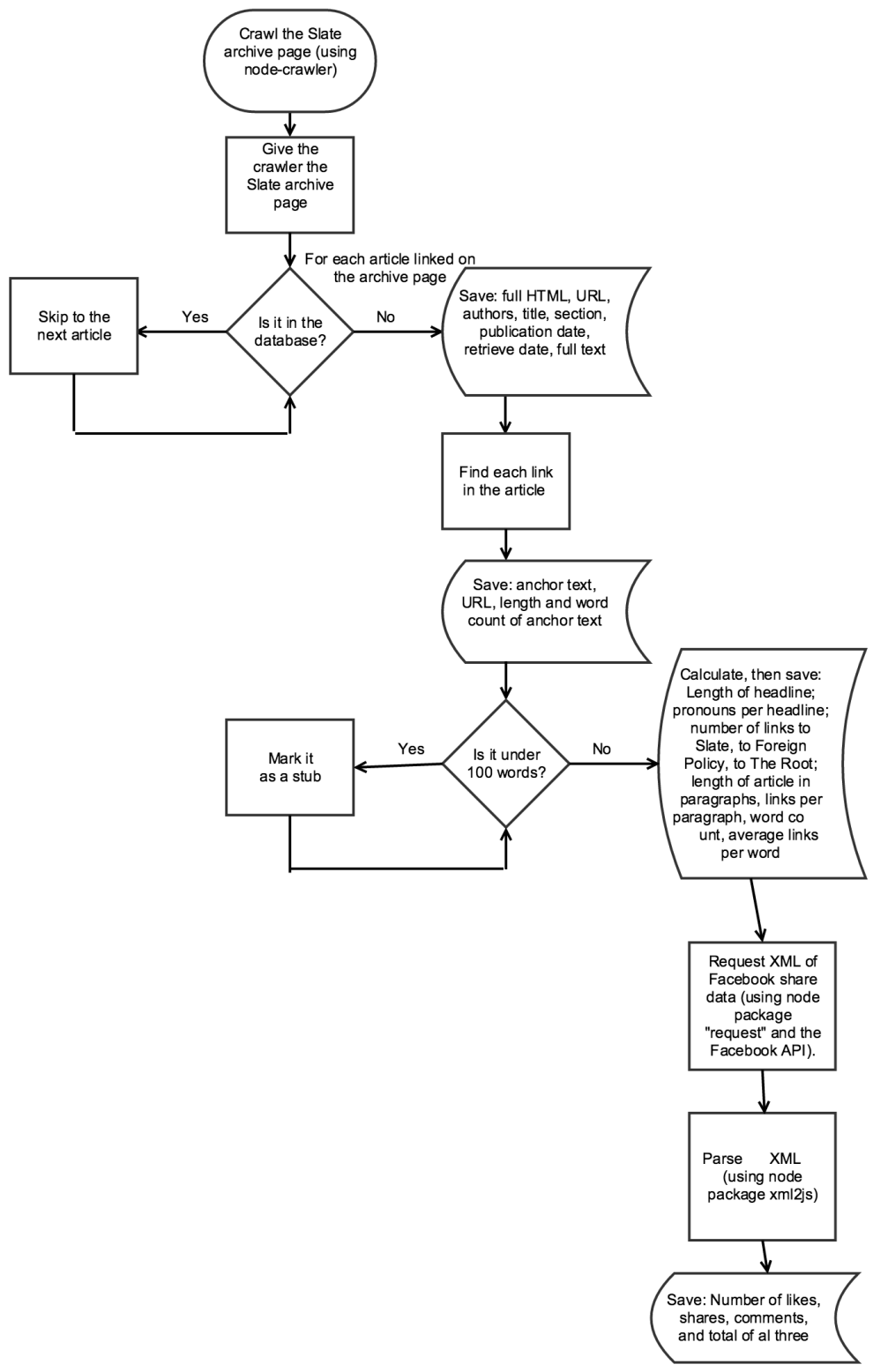

Figure 6. 1: Plain-text flowchart description of Slate crawler and associated analysis programs 


\section{CURRICULUM VITA}

NAME:

Elizabeth Frances Bergeron Chamberlain

ADDRESS: $\quad 304$ University Drive

Jonesboro, AR, 72401

DOB:

Reno, Nevada -8 September 1990

EDUCATION

\& TRAINING:

B.A., English

California State University, Los Angeles

$2003-07$

M.A., English

California Polytechnic State University, San Luis Obispo

$2008-10$

Ph.D., English/Rhetoric and Composition

University of Louisville

$2012-16$

APPOINTMENT: Assistant Professor, English

Arkansas State University, Jonesboro, AR

2016 - Present

AWARDS:

Carolyn Krause Maddox Prize in Women's \& Gender Studies, UofL

Computers and Writing Graduate Research Network Travel Award

University Fellowship, UofL

Early Entrance Program, CSULA

\section{PUBLICATIONS:}

"Buy It Yourself: How DIY Got Consumerized." Harlot: A Revealing Look at the Arts of Persuasion 14, Fall 2015

"Mess, Not Mastery: Encouraging Digital Design Dispositions in Girls." With Rachel Gramer and Megan Faver Hartline. Computers and Composition Online 
"In-Your-Field Rhetorical Analysis Assignment." Pupil: A Sourcebook for Teachers: Rhetoric and Composition 3

Review: Facebook and Philosophy, Ed. D.E. Wittkower. Kairos 19.3

\section{PRESENTATIONS:}

"Fireside Rhetoric: Using

Online Publications

Aimed at the Public to

Mobilize Rhet/Comp

Knowledge."

“'One Weird Trick':

Popular Press Strategies to Invigorate (or Infuriate)

Online Open-Access

Journals"

"Straddling the (On)line:

Newsweek, Slate, and

Citation Practices in the

Late Age of Print"

"The Risky Red Bird:

Creating University of

Louisville's Cardinal

Compositions, a Print and

Digital Journal of

Undergraduate Student

Work"

"Collaboration and

Collective Idea

Development throughout

the Writing Process"

(Digital Pedagogy Poster

Presentation)

"Modes Multiply, Navigation Calcifies:

Open-Access Genre

Change in Rhetoric and

Composition"
Thomas R. Watson

October 2016

Conference (Louisville)

Conference on College

Composition and

Communication

(Houston)

Computers and Writing

(Menomonie)

May 2015

March 2016

Conference on College

March 2015

Composition and

Communication (Tampa)
Conference on College

Composition and

Communication (Tampa)
March 2015 
"Hyperlink Economics: Online Argument and Affordances of the Link Economy"

"Narrating the 'Silent Disorder': How Mother Warriors Battled

Dominant Autism Discourse"

"DIY Subversion: The Anti-Consumerist Rhetoric of Homemade Fashion Blogs"
Conference on College

Composition and

Communication

(Indianapolis)

National Women's

Studies Association

Conference (Cincinnati)

Conference on College

Composition and

Communication (St.

Louis)
March 2014

November 2013

March 2012 